\title{
Adaptation Of The Streptococcal Collagen-Like Protein 1, Scl1, Of Group A Streptococcus To Recognize Fibronectin Type lii Repeats
}

Dudley H. Mcnitt

West Virginia University, dhmcnitt@mix.wvu.edu

Follow this and additional works at: https://researchrepository.wvu.edu/etd

Part of the Bacteria Commons, Bacterial Infections and Mycoses Commons, Bacteriology Commons, Biochemistry Commons, and the Infectious Disease Commons

\section{Recommended Citation}

Mcnitt, Dudley H., "Adaptation Of The Streptococcal Collagen-Like Protein 1, Scl1, Of Group A Streptococcus To Recognize Fibronectin Type lii Repeats" (2019). Graduate Theses, Dissertations, and Problem Reports. 3862.

https://researchrepository.wvu.edu/etd/3862

This Dissertation is protected by copyright and/or related rights. It has been brought to you by the The Research Repository @ WVU with permission from the rights-holder(s). You are free to use this Dissertation in any way that is permitted by the copyright and related rights legislation that applies to your use. For other uses you must obtain permission from the rights-holder(s) directly, unless additional rights are indicated by a Creative Commons license in the record and/ or on the work itself. This Dissertation has been accepted for inclusion in WVU Graduate Theses, Dissertations, and Problem Reports collection by an authorized administrator of The Research Repository @ WVU.

For more information, please contact researchrepository@mail.wvu.edu. 
2019

ADAPTATION OF THE STREPTOCOCCAL

COLLAGEN-LIKE PROTEIN 1, SCL1, OF

GROUP A STREPTOCOCCUS TO

RECOGNIZE FIBRONECTIN TYPE III

REPEATS

Dudley H. McNitt

Follow this and additional works at: https://researchrepository.wvu.edu/etd

Part of the Bacteria Commons, Bacterial Infections and Mycoses Commons, Bacteriology

$\underline{\text { Commons, Biochemistry Commons, and the Infectious Disease Commons }}$ 

GROUP A STREPTOCOCCUS TO RECOGNIZE FIBRONECTIN TYPE III REPEATS

\author{
Dudley Hayes McNitt \\ Dissertation submitted to the School of Medicine \\ at West Virginia University \\ in partial fulfillment of the requirements \\ for the degree of \\ Doctor of Philosophy \\ in \\ Immunology and Microbial Pathogenesis
}
Slawomir Lukomski, Ph.D., Committee Chairperson
Christopher F. Cuff, Ph.D.
F. Heath Damron, Ph.D.
Michael D. Schaller, Ph.D.
Scott A. Weed, Ph.D.

Department of Microbiology, Immunology, and Cell Biology

Morgantown, West Virginia

2019

Keywords: group A Streptococcus, streptococcal collagen-like protein 1, fibronectin, tenascin-C, adhesins, wound microenvironment, cancer microenvironment

Copyright 2019 Dudley H. McNitt 


\section{ABSTRACT}

\section{Adaptation of the streptococcal collagen-like protein 1, Scl1, of group A Streptococcus to recognize fibronectin type III repeats}

\section{Dudley H. McNitt}

Background: Group A Streptococcus (GAS) is responsible more than 700 million infections worldwide each year. Most of these infections start with initial colonization of the throat and skin, which is augmented by surface adhesins. The streptococcal collagenlike protein 1 ( $\mathrm{Scl} 1)$ is a major adhesin expressed by GAS that contains an $\mathrm{N}$-terminal sequence-variable $(\mathrm{V})$ domain, protruded away from the cell surface by the collagen domain. The Scl-V domain is comprised of three pairs of anti-parallel a-helices interconnected by surface-exposed loops. For attachment, GAS adhesins require a portal of entry, such as a wound or breach in the epithelium, to enter the body. Within the wound, host cells deposit a provisional extracellular matrix (ECM) rich in cellular fibronectin (cFn) isoforms that contain fibronectin type III (FnIII) repeats, including unique extra domains A (EDA) and $B(E D B)$, that are absent in plasma fibronectin. Tenascin-C (TnC) is another ECM component, which is substantially deposited within the wound microenvironment and contains Fnlll repeats. In addition to healing wounds, EDA/EDB-cFn and TnC are found in tumor microenvironments. Our early work showed that the Scl1-V domain binds to $\mathrm{cFn}$ via EDA. Our work here has focused on understanding this selective binding of Scl1 to wound-associated ECM and how this interaction contributes to GAS pathogenesis. Hypothesis: The Scl1-V domain has adapted the capability to bind to wound-associated FnIII repeats, EDA and EDB in cFn, and in TnC. Results: First, we discovered that surface-exposed loops of the Scl1-V domain, surrounding an acidic patch, participate in EDA binding. Both structural characteristics were conserved among phylogenetically distant Scl1 variants. We next discovered that Scl1 also binds to EDB in $\mathrm{cFn}$, as well as to the Fnlll repeats in TnC. Using defined recombinant proteins, we show that loop-region of the Scl1-V domain mediates Scl1 binding to EDB and to the TnC FnIII repeats. Moreover, Scl1-FnIII binding promotes GAS attachment and biofilm formation in 
vitro. We also developed a more complex heterogeneous in vitro matrix-system, deposited by cancer-associated fibroblasts, to demonstrate the selective binding of Scl1 to EDA/EDB-cFn isoforms and to TnC Fnlll repeats within this matrix. Conclusions: Scl1 binds to wound-associated FnIII repeats in both $\mathrm{cFn}$ and TnC. This work has implications in GAS wound-colonization and infers Scl1-Fnlll binding in modulating host woundhealing responses. This work lays a foundation for the development of strategies to target GAS during wound infections, as well as provides a rationale for the use of Scl1 in targeting cancerous tissues. 


\section{DEDICATION}

This work is dedicated to my beautiful wife, Kathleen, who has been incredibly patient, loving, and understating throughout my graduate career. Your love and support carried me through times of doubt and worry; I could not have done this without you. I look forward to the next chapters of our life together.

This is work is also dedicated to my parents, Peg and Cummins McNitt, thank you for always supporting me and believing in me. The lessons you have taught me have helped shape who I am today. 


\section{ACKNOWLEDGMENTS}

I first want to acknowledge and thank my advisor, Dr. Slawomir Lukomski. You offered me a lab to learn and grow in, and provided excellent guidance throughout my training. My success is in large part thanks to you, I will forever appreciate the time you took to help me.

I also want to acknowledge and thank our lab manager, Soo Jeon Choi. Much of this text would not exist without your hard work and without your infinite patience all those times I was frantic. Thank you for everything Soo.

Thank you to former and current lab members of the Lukosmki lab, chiefly, Dr. Beth Bachert and Megan Grund. Thank you, Beth, for being such an excellent example of a hardworking graduate student, for all your support and guidance throughout my graduate career. Thank you, Megan, for your support during my final months in the lab.

I want to acknowledge my committee members. Your suggestions and guidance on my projects and career development have been invaluable, your guidance will continue to shape the scientist I strive to be.

It takes a department to raise a graduate student. I want to thank my friends and colleagues in the department for many things: for friendly chats in the hall, providing me guidance and advice on all matters, for putting up with my impromptu visits to say hello and chat, for making work every day a joy, and so many other things. I will miss the department very much.

I want to acknowledge two frequent outside collaborators during my training, Dr. Rita Berisio and Dr. Livingston Van De Water. Both of you, and your respective labs, have provided so much support for my research, thank you. I also want to acknowledge several people at WVU who have supported me and my research in various ways. Thank you, Paolo Fagone, Dr. Ivan Martinez, Dr. Nyles Charon, and Dr. Lisa Holland.

Without my IGERT fellowship support, I would not have been able to continue in the Lukomski lab. I want to thank the National Science Foundation and the IGERT program, as well as say thank you to Dr. Kim Quedado, who guided me, and the other fellows. Thank you, Kim, for covering me during my training, especially during my last year of graduate school. I wouldn't be here without you and your willingness to champion for the IGERT fellows.

Thank you so very much to Kathleen Mills, my wife, who has provided me a shoulder to lean on, an ear to listen, hugs after a long day, and love that has helped carry me through the toughest times. I am glad I get to share this achievement with you, I can't think of anyone better.

To my parents, and to the rest of my family. Thank you for showing interest in what I do, even if I can't explain it well enough to make sense. More importantly, thank you for the love and support, the well wishes box, the phone calls, and the constant encouragement. I am very blessed.

If dogs could talk, I know three that could likely give a lecture on bacterial adhesins and the extracellular matrix. Thank you, Holly, Teddy, and Monty, for not caring how badly I messed up at the end of the day and for being much more interested in toys and walks.

Lastly, thank you to all my friends, both to those I made during my graduate training and those from before I started graduate school. Thank you for helping make this crazy journey bearable, for celebrating the achievements and helping me cope with the failures. I am a very lucky person to be surrounded by so many wonderful people. 


\section{TABLE OF CONTENTS}

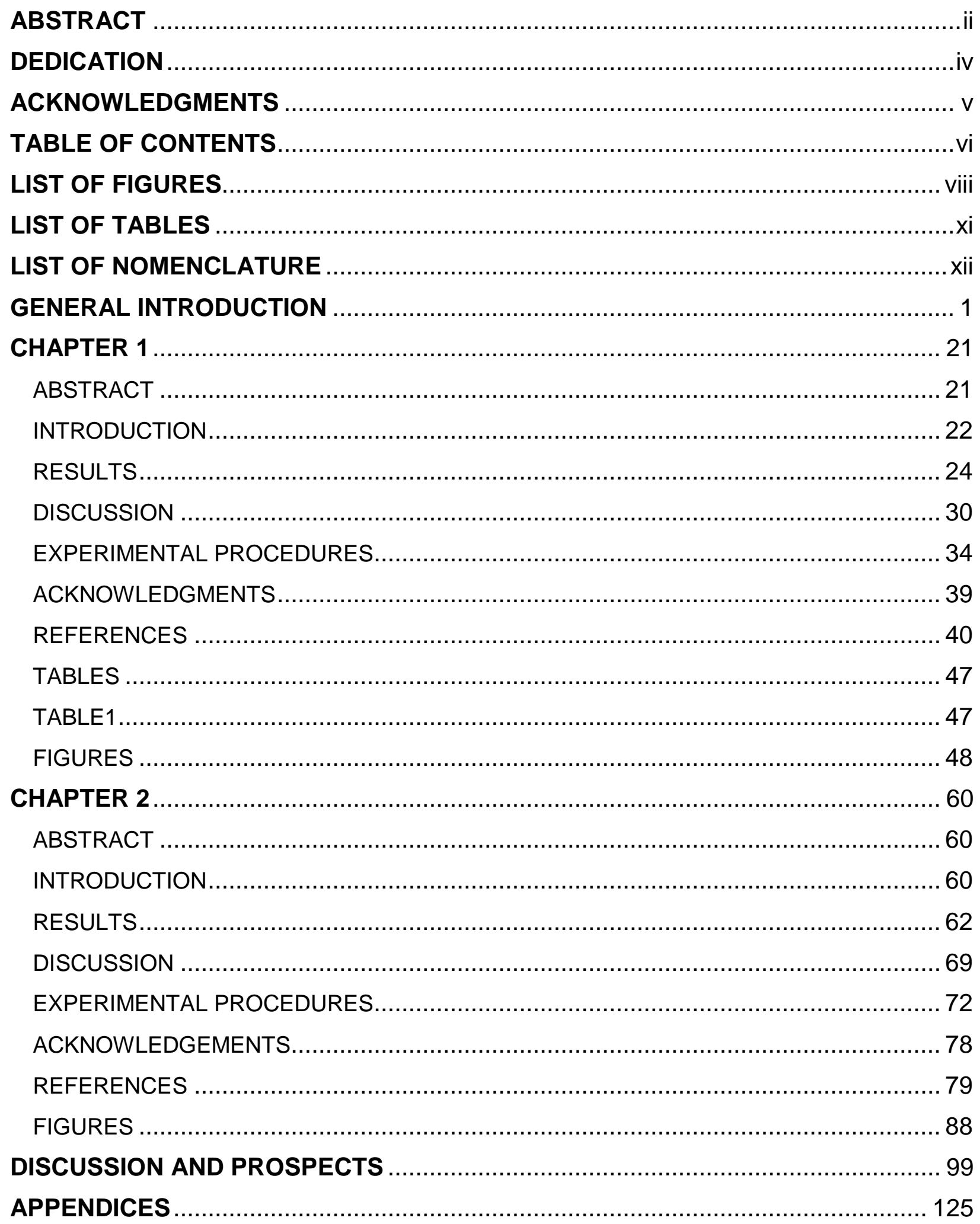




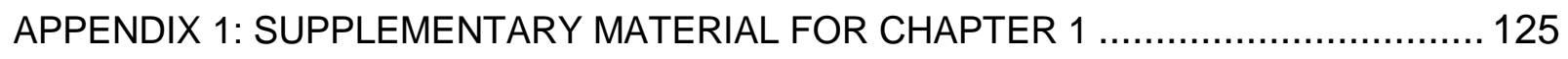

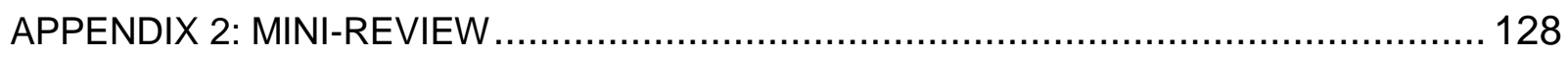

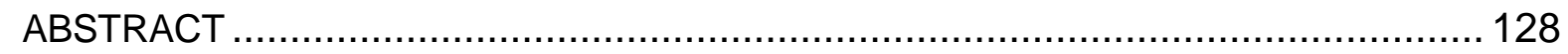

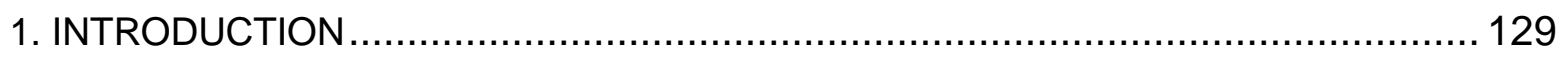

2. SCL1 AND SCL2 AS BIOMARKERS OF GAS INFECTION ................................... 130

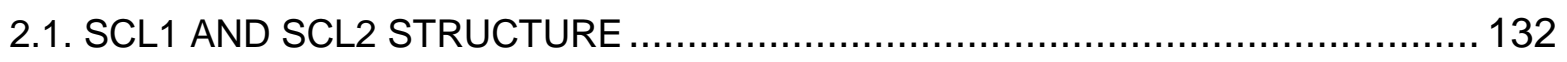

2.2. FIBRONECTIN WITHIN THE WOUND MICROENVIRONMENT ......................... 134

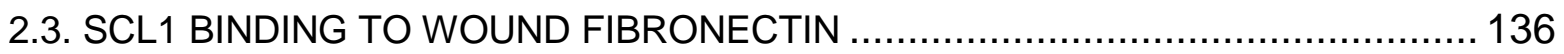

3. DIFFERENT STRATEGIES FOR TARGETING GAS INFECTIONS ......................... 136

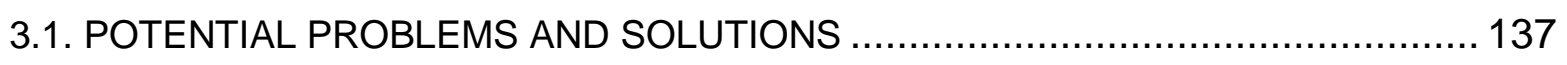

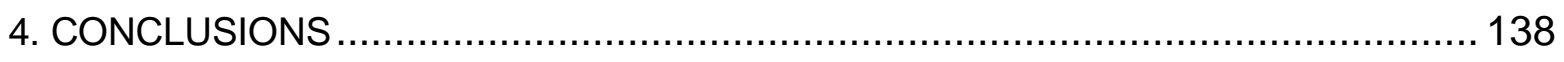

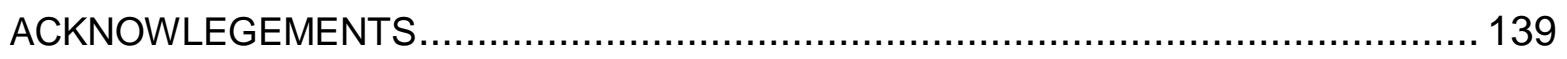

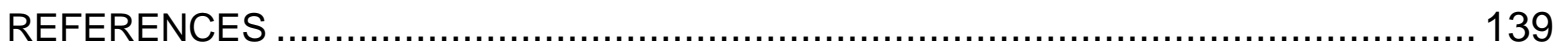

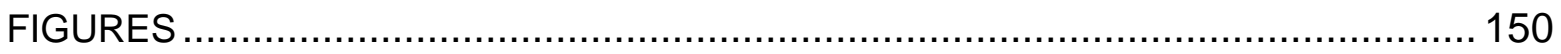

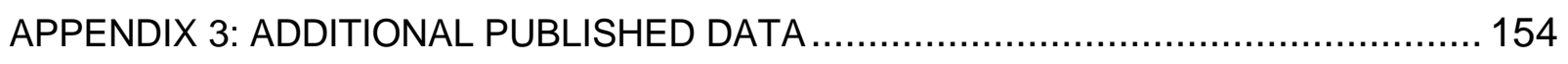

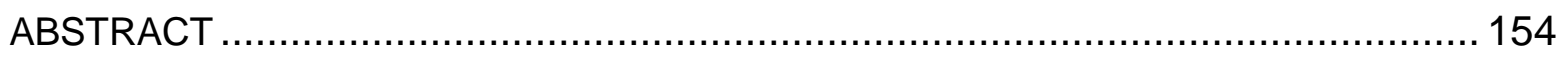

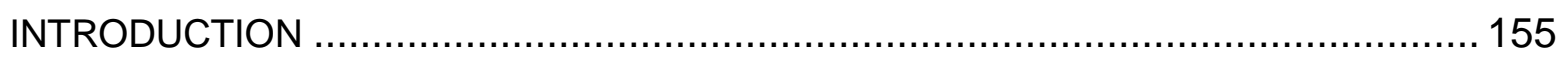

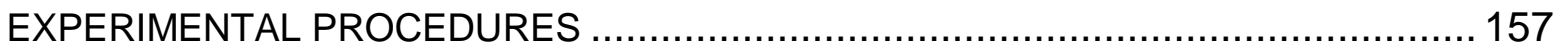

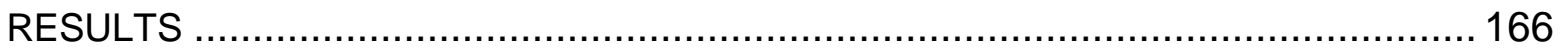

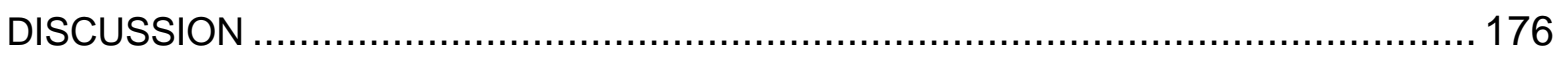

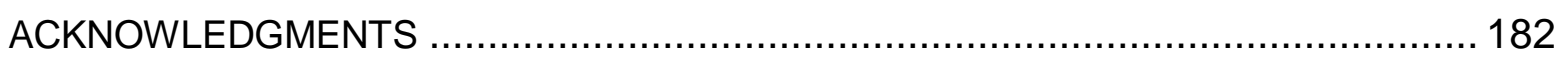

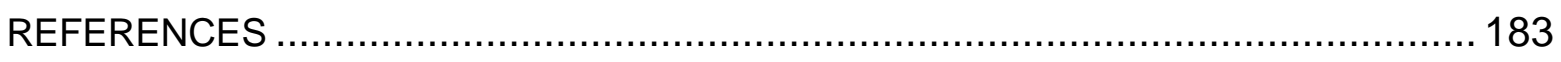

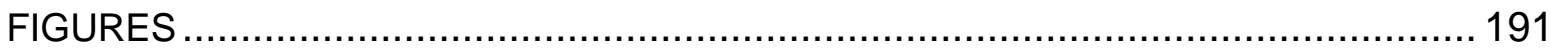

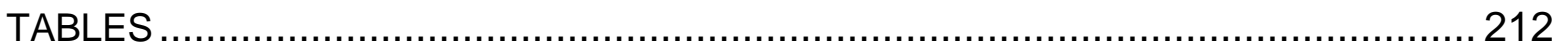




\section{LIST OF FIGURES}

\section{General}

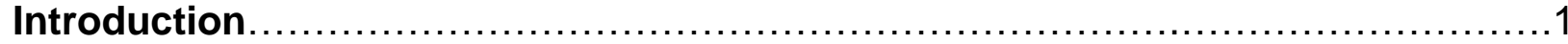

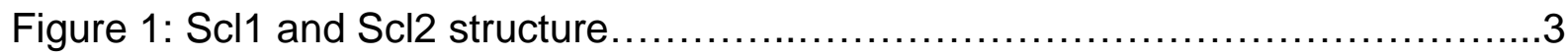

Figure 2: Scl1 binds to cellular fibronectin that contains extra domain $\mathrm{A} \ldots \ldots \ldots \ldots \ldots . . . . .5$

Chapter 1 - Surface-exposed loops and an acidic patch in the Scl1 protein of group A Streptococcus enable Scl1 binding to wound-associated fibronectin.....................21

Figure 1: Conserved structure of the Scl1 and Scl2 globular domains...............48

Figure 2: Full-length Scl1 proteins are required for binding to ECM ligands...........50 Figure 3: Surface-exposed loop of the Scl1.1-V domain is central in ECM recognition and binding

Figure 4: The C-terminal 11 amino acids of the loop segment of the Scl1.1-V domain is central in ECM recognition and binding. .53

Figure 5: Electrostatic potential surfaces of Scl1-V, Scl2-V, and Scl.chi-V domains..55 Figure 6: Conservation of ECM recognition and binding by phylogenetically distant Scl1 variant.

Figure 7: Conserved structural constraints and exposed loop of Scl1 proteins determine binding to laminin and apolipoprotein B100/low-density lipoprotein..... .58

Chapter 2 - Adaptation of the group A Streptococcus adhesin Scl1 to bind fibronectin type III repeats within wound-associated extracellular matrix: implications in cancer targeting

Figure 1: Scl1-EDB binding mediates GAS adherence and biofilm formation.........88

Figure 2: Scl1 of GAS binds to the fibronectin type III repeats in tenascin $\mathrm{C}(\mathrm{TnC}) \ldots 90$

Figure 3: Characterization of extracellular matrices deposited by cancer-associated fibroblasts (CAFs).

Figure 4: Scl1-mediated GAS attachment to and biofilm formation on ECM deposited

by cancer-derived fibroblasts.

Figure 5: Scl1-mediated GAS attachment to and biofilm formation on ECM deposited by bone osteosarcoma cells. .95

Figure 6: Model of GAS colonization of wound and tumor microenvironments. .97 


\section{Discussion and Prospects}

Figure 1: Modulation of host responses by GAS Scl1 during wound colonization....102

Figure 2: Monobodies to target GAS via Scl1 .....................................109

Figure 3: Specificity of Scl1-mediated GAS attachment to cancer-associated fibroblast matrices.

Figure 4: Hybrid rScl protein in nanoparticle delivery to tumors.....................112

\section{Appendices}

Appendix 2 - Mini-Review: Streptococcal Collagen-like Protein 1 Binds Wound

Fibronectin: Implications in Pathogen Targeting........................................128

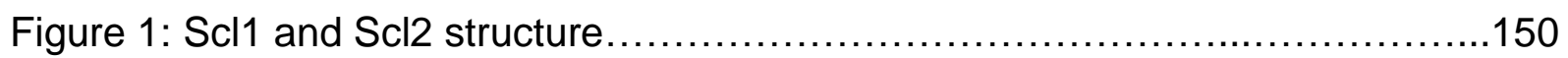

Figure 2: Scl1 binds to cellular fibronectin that contains extra domain A.............152

Figure 3: Modulation of host responses by GAS Scl1 during wound colonization....153 Appendix 3 - Additional Published Data: Unique footprint in the sc/1.3 locus affects adhesion and biofilm formation of the invasive M3-type group A Streptococcus........154

Figure 1: M3-type GAS strains harbor unique polymorphisms in the scl1.3 locus...191

Figure 2: Assessment of Scl1.3 expression......................................193

Figure 3: Characterization of the scl2.3 locus in M3-type GAS .....................195

Figure 4: In vitro skin equivalent model of GAS

infection. $196-197$

Figure 5: In vitro biofilm formation and hypothetical model of microcolony formation by invasive M3-type GAS 198-199

Figure 6: Construction and binding characterization of recombinant full-length

Scl1.3FL and Scl2.3 proteins. 200

Figure 7: Homologous complementation of M3-type GAS with full-length surfaceexpressed Scl1.3 protein confers biofilm formation. 202

Figure 8: Heterologous complementation of scl1-deficient mutant of M41-type GAS with full-length Scl1.3 restores biofilm formation 204

Figure 9: Skin pathology of SKH1 hairless mice infected subcutaneously with wildtype and sc/1-inactivated mutants of M28- and M41-type GAS .206 
Figure 10: Skin pathology of SKH1 hairless mice infected subcutaneously with wildtype and sc/1-inactivated mutants of M28- and M41-type GAS.....................207 Figure S1: Analytical PCR of $s c / 1.3$ and $s c / 2.3$ in M3-type GAS ....................208 Figure S2: Domain organization of rScl1.3FL and rScl2.3 proteins.................209 Figure S3: Colocalization of glycocalyx staining with GFP-expressing M41-type GAS

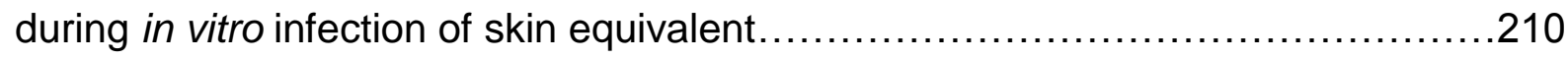
Figure S4: Strategy for C-C' loop peptide design .................................211 


\section{LIST OF TABLES}

Chapter 1 - Surface-exposed loops and an acidic patch in the Scl1 protein of group A Streptococcus enable Scl1 binding to wound-associated fibronectin....................21

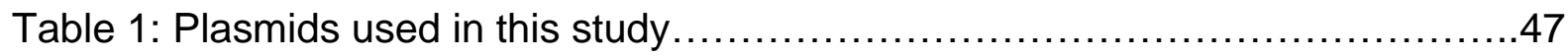

\section{Appendices}

Appendix 1 - Chapter 1: Surface-exposed loops and an acidic patch in the Scl1 protein of group A Streptococcus enable Scl1 binding to wound-associated fibronectin |

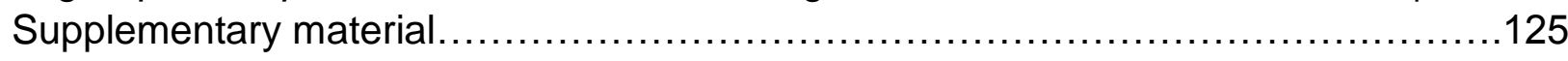

Table S1: Synthetic double stranded DNA blocks (gBlocks) and primer nucleotide sequences used in this study

Appendix 3 - Additional Published Data: Unique footprint in the sc/1.3 locus affects adhesion and biofilm formation of the invasive M3-type group A Streptococcus. 154

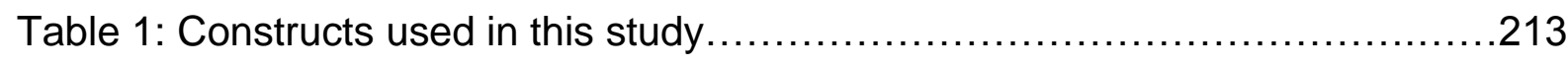

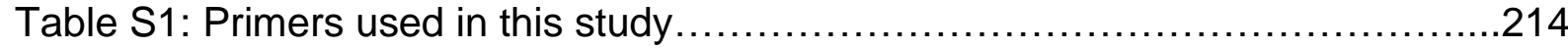

Table S2: Variation in scl2.3 gene among M3 strains ............................215

Table S3: Mass spectrometry identification of $\mathrm{rScl}$ and native Scl proteins..........216

Table S4: Summary table of fold-changes in sc/1 and scl2 expression...............217 


\section{LIST OF NOMENCLATURE}

1. GAS: Group A Streptococcus

2. ECM: Extracellular matrix

3. Scl1, Scl2: Streptococcal collagen-like protein 1 and 2

4. $\underline{V}$ : Variable domain

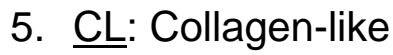

6. Fn: Fibronectin

7. pFn: Plasma fibronectin

8. $\underline{\mathrm{cFn}}$ : Cellular fibronectin

9. EDA/EIIIA: Extra domain A - EDA/cFn: $c F n$ isoforms that contain EDA

10. EDB/EIIIB: Extra domain $B-E D B / c F n: ~ c F n$ isoforms that contain EDB

11. Fnlll: Fibronectin type III repeat

12. TnC: Tenascin-C

13. TnFnIII: Tenascin-C fibronectin type III repeats

14. LDL: Low density lipoprotein

15. rScl: Recombinant streptococcal collagen-like protein

16. rScl.chi: Recombinant chimeric streptococcal collagen-like protein

17. apoB100: Apolipoprotein B100

18. rScl1FL: Full-length recombinant streptococcal collagen-like protein 1

19. rScl1V: Variable domain-only streptococcal collagen-like protein 1

20. $\triangle s c / 1$ : $s c / 1$-inactivated mutant

21. rEDA: Recombinant extra domain A

22. rEDB: Recombinant extra domain $B$

23. CAFs: Cancer-associated fibroblasts 


\section{GENERAL INTRODUCTION \\ FIBRONECTIN BINDING PROTEINS OF GAS: UNIQUE ADHERENCE OF THE SCL1 ADHESIN TO THE WOUND MICROENVIRONMENT}

(Portions of the introduction have been published in a mini-review; see appendix 2)

\section{Group A Streptococcus epidemiology}

Group A Streptococcus (GAS) is a human-adapted pathogen that is responsible for over 700 million infections worldwide each year (1). Diseases linked to GAS infection range in severity from non-invasive superficial pharyngitis and impetigo to severe and invasive diseases like necrotizing fasciitis or streptococcal toxic shock syndrome (1). Additionally, post-infectious autoimmune sequelae include rheumatic fever and rheumatic heart disease, acute poststreptococcal glomerulonephritis, and pediatric autoimmune neurologic disorder associated with streptococci, also known as PANDAS (2-4). Invasive and autoimmune diseases account for approximately 500,000 deaths globally each year (1). GAS can transiently colonize barrier tissue sites, such as throat mucosa and the surface of the skin (5). When the barrier is breached, the resultant wound provides GAS with a portal of entry into host tissues and the interstitium. Once inside the wound, GAS adheres to exposed extracellular matrix (ECM) and cells, thus enabling colonization of the host. These essential first steps of infection that are critical to colonization are augmented by several GAS surface adhesins, such as a prominent GAS surface adhesin and key virulence factor, the M protein (6). DNA sequence encoding the outermost aminoterminal end of the M protein is hypervariable and is used for GAS typing, resulting in more than $220 \mathrm{emm}$ types of GAS reported globally (7). Two other major GAS surface proteins are the streptococcal collagen-like proteins 1 (Scl1/SclA) and 2 (Scl2/SclB), which are ubiquitous among GAS strains (8-12).

\section{The streptococcal collagen-like proteins}

Both scl1 and scl2 genes have been identified in many epidemiologically important $\mathrm{M}$ types of GAS $(8-11,13)$. sc/1 expression is positively regulated by the multiple-gene regulator, Mga $(8,9,14)$. Mga is a major virulence-associated transcriptional regulator expressed in all GAS strains (15-18). It controls the expression of key GAS virulence 
factors, including the $M$ and M-like proteins, C5a peptidase, Sic, and Scl1 (8,9,19-21). Elevated transcription of $s c / 1$ has been found in GAS isolates from both non-invasive and invasive infections $(13,22)$. Unlike Scl1, expression of full-length Scl2 protein is controlled during polypeptide translation by phase variation, which is associated with varying numbers of pentanucleotide CAAAA repeats located downstream of the scl2-start codon (9-11). Like sc/1, scl2 transcription has been observed during GAS infection, but protein expression varies between strains due to the aforementioned phase variation $(9,23)$. The invasive M3-type GAS strains (24-26) show elevated levels of sc/2 transcripts and low levels of scl1 transcripts, compared with strains of several other M types (27).

Both Scl1 and Scl2 proteins have similar structural organization (28) (Fig. 1A). Both are homotrimeric, with an $\mathrm{N}$-terminal globular, sequence-variable $(\mathrm{V})$ domain, followed by the collagen-like $(\mathrm{CL})$ domain and a $\mathrm{C}$-terminal cell wall-anchoring domain. The $\mathrm{CL}$ domain of both Scl1 and Scl2 is composed of varying numbers of Gly-Xaa-Yaa collagen repeats, analogous to triple-helical mammalian collagen $(29,30)$. Structurally, the triple helix is a motif characterized by the association of three distinct polypeptide chains wrapped around a common axis, where each chain assumes a polyproline-II (PPII)-type conformation (31-34). However, the CL domain does not contain hydroxyproline, which provides the main stabilizing contribution to triple-helix structure in mammalian collagens (35-39). Indeed, bacteria lack the prolyl-hydroxylase, an enzyme necessary for posttranslational modification of Pro to Hyp. Despite the lack of Hyp, however, Scl1- and Scl2$\mathrm{CL}$ domains form triple-helix structures with a stability ranging between 35 and $40^{\circ} \mathrm{C}$, similar to mammalian collagens (29,40-43). Instead, alternative stabilization mechanisms are employed in bacteria that secure triple-helix stability, such as hydration networks, electrostatic interactions between side chains, and specific stabilization repeats $(30,43)$.

The primary sequences of $\mathrm{V}$ domains differ both between and within Scl1 and Scl2 variants originating from strains of different $M$ types (28). Despite sequence differences, secondary-structure prediction algorithms identified two conserved $\alpha$-helices interspaced by a hypervariable segment $(8,44)$. This prediction was validated by crystallography data of the recombinant protein, corresponding to the Scl2-V domain from an M3-type GAS $(45,46)$ (Fig. 1A). The crystal structure showed that the Scl2-V domain folds into a trimeric six-helical bundle, forming a cylinder that is $30 \AA$ in diameter and $60 \AA$ in height, which is 
stabilized by a hydrophobic core and salt bridges. The inner core of this bundle consists of a parallel, trimeric structure in which helices are wrapped in a gradual left-handed superhelix. Three further helices wrap antiparallel to the internal helices in a left-handed

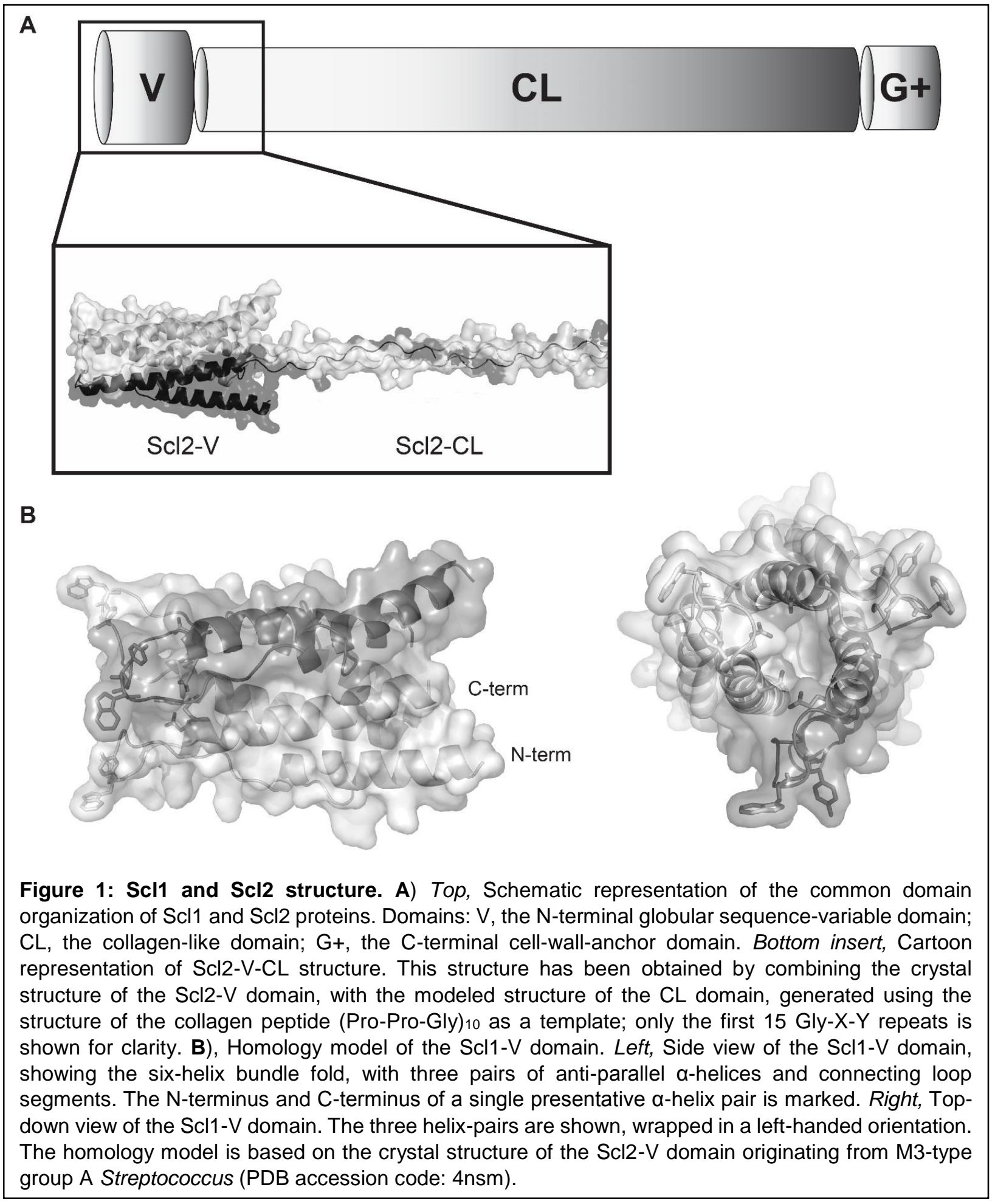


direction around the exterior of the central trimer. The pairs of anti-parallel $\alpha$-helices are each connected with PPII-type loop segments. Structural and biophysical data suggested that $\mathrm{V}$ domains of Scls display dichotomous functions by acting as triple-helix stabilization domains through facilitating proper folding and refolding of the collagen triple-helix (47). Consistently, recombinant collagen-domain constructs cannot refold following thermal denaturation $(29,47)$. In addition to facilitating trimerization of the CL-domain, the Vdomain of Scl1 mediates host-pathogen interaction (48-50).

Scl1 binds to several ligands found in tissue and blood, compared with Scl2; the latter's function in GAS pathogenesis is less clear (reviewed in (28)). The collagen domain of Scl1 can bind and activate human collagen integrin receptors $(48,49,51)$, whereas the $\mathrm{V}$ domain is responsible for the majority of ligand binding (44,51-55). Interestingly, Scl1 mutants of M1-type GAS were attenuated in a mouse skin infection model, but not following intraperitoneal inoculation (12), conceivably due to differences in the capacity to evade innate immune defenses between wild type and mutant strains $(12,56)$. In contrast, Scl1 mutants of two other M types exhibited increased lesion size in mouse skin (27). In vitro assays and animal models show that Scl1 contributes to host colonization and biofilm formation within tissues by binding to host ECM proteins $(27,52,57)$. Scl1 binds to wound-enriched isoforms of cellular fibronectin containing the extra domain $A$ (58). The ubiquitous presence of Scl1 in GAS strains across different M types and the distinctive binding to cellular fibronectin isoforms specific to the wound microenvironment, which enables favorable colonization of the first environment encountered by GAS during infection.

\section{Extracellular matrix proteins associated with the wound microenvironment}

GAS utilizes a wounded portal of entry to gain access to host tissues, initiating host colonization and subsequent infection. One of the essential measures during wound healing is the deposition of new distinct matrices that participate in tissue remodeling and signal pro-inflammatory responses. These complex processes engage macrophages and neutrophils in clearing out tissue debris and keratinocytes in facilitating wound closure 
$(59,60)$. One of the major constituents of these matrices is fibronectin (Fn) (61-63). Fn is a high-molecular weight glycoprotein that exists in two main forms within the body, e.g., as a plasma fibronectin ( $\mathrm{pFn}$ ) secreted by hepatocytes into the blood stream and as

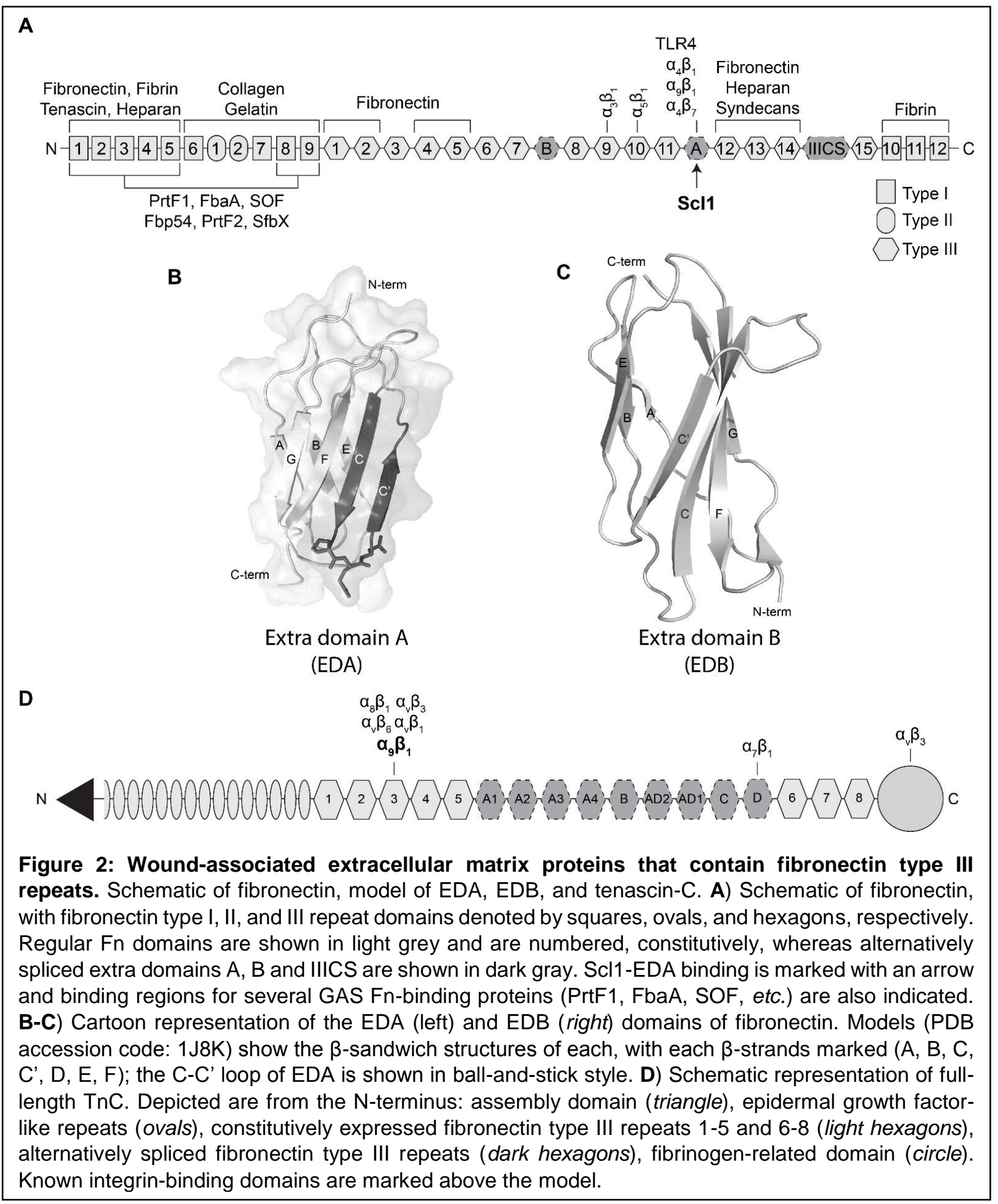


cellular fibronectin (cFn) secreted by cells within tissues (64). Both forms of fibronectin originate from the same gene, FN1, and are composed of three different types of repeating domains: types I, II, and III $(64,65)$ (Fig. 2A). cFn-mRNA can be alternatively spliced, resulting in more than 20 different isoforms found within the body (65-67). This alternative splicing leads to the inclusion of extra domains that are found in $\mathrm{cFn}$, but not pFn. The alternatively spliced type III domains are known as extra domain A (EDA/EIIIA) and extra domain $B$ (EDB/EIIIB), as well as different combinations of the type III connecting segment (IIICS), also known as the variable domain (67).

The EDA-containing $\mathrm{cFn}$ isoforms (EDA/cFn) are highly expressed during embryonic development but only at negligible levels within healthy adult tissues (68-70). On the other hand, EDA/cFn isoforms are deposited in high quantities within inflamed and wounded tissue environments in adults and play essential roles in wound healing $(69,71$ 73). EDA adopts the prototypical type III repeat structure, which is composed of two $\beta$ sheets, forming a $\beta$-sandwich consisting of seven $\beta$-strands (i.e. $A, B, C, C^{\prime}, D, E$, and $F$ ) connected by loop segments (74,75)(Fig. 2B). In full-thickness excisional wounds, wound healing was delayed in mice lacking EDA, however, others report no differences in wound healing between normal and EDA knockout mice $(72,76)$. These differences may be due to differences in genetic background of the mice used in each study, in wounding method or differences in analysis. Other studies in mice found deletion of EDA protected mice from developing lung fibrosis during murine models of pulmonary fibrosis (77), and when crossing EDA knockout mice to ApoE knockout mice, EDA deletion results in smaller atherosclerotic lesions compared to control mice (78). Dysregulation of EDA splicing, resulting in EDA overexpression, results in increased occlusive thrombi during vascular damage compared to normal mice (79), and EDA overexpression results in differences in behavior compared to normal mice (80).

An important role for EDA/cFn within wounded tissue is to support host cell attachment, achieved by the interactions between the C-C' loop of EDA and cellular integrin receptors, $\alpha_{9} \beta_{1}$ and $\alpha_{4} \beta_{1}$, as well as $\alpha_{4} \beta_{7}$, which binds EDA by an unknown mechanism (81-83). Integrin $\alpha_{9} \beta_{1}$ is upregulated by keratinocytes during tissue injury $(71,84)$. Integrins $\alpha_{9} \beta_{1}, \alpha_{4} \beta_{1}$, and $\alpha_{4} \beta_{7}$ through their attachment to EDA/cFn regulate 
important repair mechanisms, such as cell migration, angiogenesis, myofibroblast differentiation, growth factor secretion, and tissue remodeling $(71,73,83,85,86)$.

Like EDA, EDB also adopts the prototypical type III repeat structure (Fig. 2C), and follows the same expression pattern as EDA, highly expressed during embryogenesis and development, but only found in wounded and inflamed adult tissues $(69,87)$. Isoforms of $\mathrm{cFn}$ that contain EDB are highly expressed in the cerebral spinal fluid of patients suffering from bacterial meningitis, compared to non-bacterial meningitis (88). The canonical role of EDB is its association with angiogenesis, as it is highly expressed around growing vasculature in human tissues during fetal tissue development, in the granulation tissue of wounds, and within the stroma around tumors, suggesting an important role for EDB containing cFn isoforms in the growth of blood vessels (89). Despite this known association, the role of EDB during wound healing is not as well understood as that of EDA, as no direct cell binding sites have been identified within EDB (84). EDB knockout mice do not exhibit noticeable differences compared to normal mice in studies examining behavior, angiogenesis, tumorigenesis, and bone fracture healing (90-92). The lack of noticeable changes in these systems may be due to compensation of EDA or other ECM proteins (91). Double knockout of EDA and EDB is embryonic lethal in mouse models, exhibiting $80 \%$ penetrance, with the surviving pups exhibiting severe cardiovascular defects (93), suggesting that EDA and EDB may compensate for each other when one domain is unable to be expressed. Some studies have however identified important roles for EDB in fibronectin structure, using in vitro or ex vivo samples. Inclusion of EDB in cFn leads to confirmation changes that expose cryptic sites that facilitate cell attachment to cFn (94). In addition, EDB isoforms of cFn contributes to head-to-tail dimerization of cFn, facilitating Fn fibril formation on the surface of cells, supported by the observation that mouse embryonic fibroblasts from EDB knockout mice deposit smaller Fn fibrils than those from normal mice $(90,95)$. Though EDA and EDB are fibronectin type III repeats (Fnlll repeats) with highly regulated expression, other ECM proteins within the wound microenvironment also contain Fnlll repeats.

Tenascin-C $(\mathrm{TnC})$ is another ECM protein that is highly expressed within the wound microenvironment, as a component of the provisional wound matrix, and contains Fnlll repeats (96). TnC is high molecular weight glycoprotein, and is the founding member 
of the Tenascin family, that also consists of Tenascin-R, Tenascin-X, and Tenascin-W (97). TnC is comprised of distinct regions, starting from the N-terminus, the heptad repeats, followed by the epidermal growth factor-like repeats, the FnIII repeats, and a Cterminal fibrinogen-related domain (Fig. 2D)(98). The Fnlll repeats of TnC adopt the prototypical FnIII $\beta$-sandwich structure, as well as undergo alternative splicing, where the first 5 and last 3 repeats are constitutively expressed, and a varying number and combination of 9 different Fnlll repeats, in humans, can be included between repeats 5 and $6(97,99)$. This results in the potential for over 511 different isoforms of $\mathrm{TnC}$ to be expressed in tissues, though only 100 variants have been identified in tissues (97). Though both Fn and TnC contain Fnlll repeats, they originate from different genes, FN1 and TNC, respectively $(100,101)$. Genetic analysis of animals from different phylum reveal that while fibronectin was discovered first, TnC appears first in evolution (100).

$\mathrm{TnC}$ is often co-expressed with $\mathrm{Fn}$ in tissues, circulating in blood plasma at low levels, but highly expressed in blood and tissues upon inflammation, similar to EDA and EDB isoforms of cFn $(96,102)$. Upon inflammation and tissue damage, TnC is deposited into the wound from blood plasma, co-localizing with fibronectin fibrils in the provisional ECM $(103,104)$. At the same time, TnC mRNA is upregulated within the first 24 hours of wounded tissue cells such as keratinocytes and fibroblasts, and is deposited during this time (105-107). TnC protein levels will grow into their peak during the beginning of tissue building and repair phases of wound healing, deposited by activated fibroblasts, and remain high in the tissues for several days to weeks pending the anatomical site and nature of the injury (96). As a part of the ECM, TnC plays multiple roles, such as supporting other proteins within the ECM, regulating cell attachment to other ECM proteins, initiating inflammation via direct binding to TLR4, and is directly bound by cells to facilitate wound closure (108) (109-112). The Fnlll repeats of TnC are directly bound by cells via integrin receptors, due in part to the presence of the RGD motif and an IDG motif in the third TnC FnIII repeat, bound by $\alpha_{v} \beta_{1}$ and $\alpha_{9} \beta_{1}$ respectively (TnFnIII)(Fig. 2D) (113-115). These, and other integrin binding interactions, were found to mediate cell motility and survival (115), and are believed to be critical for proper wound healing through facilitating keratinocyte and fibroblast proliferation and migration and regulating attachment to collagen type I fibers $(96,107,116,117)$. In addition to regulating host cell 
attachment, TnC Fn III fragments also regulate ECM assembly through direct binding to proteoglycans and play important roles in tissue remodeling through mediating myofibroblast wound contracture $(118,119)$.

\section{Fibronectin-binding proteins of GAS}

In addition to being a target for host cells, TnC is bound by several pathogens, including GAS, as GAS expresses several adhesins that bind to both TnC $(120,121)$ and Fn (122), though, there are more examples of those that bind to Fn.

GAS expresses a number of fibronectin binding proteins, which can be broadly divided into two types, those with fibronectin-binding repeat domains, and those without. $(122,123)$. The fibronectin-binding repeats are found in several fibronectin-binding proteins of GAS, including Protein F1, Protein F2, Serum opacity factor, and fibronectinbinding protein A (124-129). The unstructured fibronectin-binding repeats of these adhesins bind to the $\mathrm{N}$-terminal $\mathrm{Fnl}$ repeats, through a tandem $\beta$-zipper mechanism $(125,130)$. The Fnl repeats adopt a $\beta$-hairpin, a three-stranded antiparallel $\beta$-sheet. Upon contact with the $\mathrm{Fnl}$ repeats, the unstructured region(s) of these fibronectin-binding repeats fold into a $\beta$-strand structure, and this strand will accent the stacked $\beta$-strands in the Fnl repeat $(125,130)$. Through binding to the type I repeats of Fn, these adhesins contribute to GAS adherence to both $\mathrm{pFn}$ and $\mathrm{cFn}$. These types of repeats have been suggested to bind to the FnIII repeats (121), however, no data exists as of this writing that demonstrates direct binding to the FnIII repeats by any GAS fibronectin-binding protein that contains these unstructured repeats. Several of these adhesins have been shown to contribute to both host colonization, as well as to intracellular entry of GAS into cells via a bridge mechanism, where GAS adheres to the Fnl repeats that are subsequently internalized via endocytosized due to interactions between host-cell integrin receptors (e.g. $\left.\alpha_{5} \beta_{1}\right)$ and the FnIII repeats $(131,132)$, consequently internalizing the attached GAS. Deletion of several of these adhesins in GAS has shown to result in decreases in celladherence and virulence compared to WT strains $(129,133,134)$.

Scl1 of GAS falls into the second category of adhesin, as Scl1 proteins across M types do not contain any fibronectin-binding repeat domains (12). Adhesins in this category are known to bind within the type I, II, and III repeats of Fn (122), however, Scl1 
is the only adhesin, as of this writing, shown to adhere to the wound-associated alternatively spliced Fnlll repeat, EDA, of cFn (58).

\section{REFERENCES}

1. Carapetis, J. R., Steer, A. C., Mulholland, E. K., and Weber, M. (2005) The global burden of group A streptococcal diseases. Lancet Infect. Dis. 5, 685-694

2. Bisno, A. L., Pearce, I. A., Wall, H. P., Moody, M. D., and Stollerman, G. H. (1970) Contrasting epidemiology of acute rheumatic fever and acute glomerulonephritis. N. Engl. J. Med. 283, 561565

3. Swedo, S. E., Leonard, H. L., Mittleman, B. B., Allen, A. J., Rapoport, J. L., Dow, S. P., Kanter, M. E., Chapman, F., and Zabriskie, J. (1997) Identification of children with pediatric autoimmune neuropsychiatric disorders associated with streptococcal infections by a marker associated with rheumatic fever. Am. J. Psychiatry 154, 110-112

4. Cunningham, M. W. (2000) Pathogenesis of group A streptococcal infections. Clin. Microbiol. Rev. 13, 470-511

5. Bessen, D. E., and Lizano, S. (2010) Tissue tropisms in group A streptococcal infections. Future Microbiol. 5, 623-638

6. Fischetti, V. (2016) M protein and other surface proteins on streptococci. In Streptococcus pyogenes: Basic biology to clinical manifestations [Internet] (Ferretti JJ, S. D., Fischetti VA ed.), Univeristy of Oklahoma Health Sciences Center, Oklahoma City, OK.

7. Sanderson-Smith, M., De Oliveira, D. M., Guglielmini, J., McMillan, D. J., Vu, T., Holien, J. K., Henningham, A., Steer, A. C., Bessen, D. E., Dale, J. B., Curtis, N., Beall, B. W., Walker, M. J., Parker, M. W., Carapetis, J. R., Van Melderen, L., Sriprakash, K. S., and Smeesters, P. R. (2014) A systematic and functional classification of Streptococcus pyogenes that serves as a new tool for molecular typing and vaccine development. J. Infect. Dis. 15; 1325-38

8. Rasmussen, M., Edén, A., and Björck, L. (2000) SclA, a novel collagen-like surface protein of Streptococcus pyogenes. Infect. Immun. 68, 6370-6377

9. Lukomski, S., Nakashima, K., Abdi, I., Cipriano, V. J., Shelvin, B. J., Graviss, E. A., and Musser, J. M. (2001) Identification and characterization of a second extracellular collagen-like protein made by group A Streptococcus: control of production at the level of translation. Infect. Immun. 69, 1729-1738

10. Rasmussen, M., and Björck, L. (2001) Unique regulation of SclB - a novel collagen-like surface protein of Streptococcus pyogenes. Mol. Microbiol. 40, 1427-1438

11. Whatmore, A. M. (2001) Streptococcus pyogenes sclB encodes a putative hypervariable surface protein with a collagen-like repetitive structure. Microbiology 147, 419-429 
12. Lukomski, S., Nakashima, K., Abdi, I., Cipriano, V. J., Ireland, R. M., Reid, S. D., Adams, G. G., and Musser, J. M. (2000) Identification and characterization of the sc/ gene encoding a group $A$ Streptococcus extracellular protein virulence factor with similarity to human collagen. Infect. Immun. 68, 6542-6553

13. Sumby, P., Whitney, A. R., Graviss, E. A., DeLeo, F. R., and Musser, J. M. (2006) Genome-wide analysis of group A streptococci reveals a mutation that modulates global phenotype and disease specificity. PLoS Pathog. 2, e5

14. Almengor, A. C., and Mclver, K. S. (2004) Transcriptional activation of $s c / A$ by Mga requires a distal binding site in Streptococcus pyogenes. J. Bacteriol. 186, 7847-7857

15. Spanier, J. G., Jones, S. J., and Cleary, P. (1984) Small DNA deletions creating avirulence in Streptococcus pyogenes. Science 225, 935-938

16. Podbielski, A. (1992) Ubiquitous occurrence of virR and $s c p A$ genes in group A streptococci. Med. Microbiol. Immunol. 181, 227-240

17. Scott, J. R., Cleary, P., Caparon, M. G., Heden, K. M. L., Musser, J. M., Hollingshead, S., and Podbielski, A. (1995) New name for the positive regulator of the M protein of group A Streptococcus. Mol. Microbiol. 17, 799

18. Bessen, D., Manoharan, A., Luo, F., Wertz, J., and Robinson, D. (2005) Evolution of transcription regulatory genes is linked to niche specialization in the bacterial pathogen Streptococcus pyogenes. J. Bacteriol. 187, 4163-4172

19. Caparon, M. G., and Scott, J. R. (1987) Identification of a gene that regulates expression of M protein, the major virulence determinant of group A streptococci. Proc. Natl. Acad. Sci. U. S. A. 84, 8677-8681

20. Hoe, N. P., Nakashima, K., Lukomski, S., Grigsby, D., Liu, M., Kordari, P., Dou, S.-J., Pan, X., Vuopio-Varkila, J., Salmenlinna, S., McGeer, A., Low, D. E., Schwartz, B., Schuchat, A., Naidich, S., De Lorenzo, D., Fu, Y.-X., and Musser, J. M. (1999) Rapid selection of complement-inhibiting protein variants in group A Streptococcus epidemic waves. Nat. Med. 5, 924-929

21. Podbielski, A. (1993) Three different types of organization of the vir regulon in group A streptococci. Mol. Gen. Genet. 237, 287-300

22. Tsatsaronis, J. A., Hollands, A., Cole, J. N., Maamary, P. G., Gillen, C. M., Ben Zakour, N. L., Kotb, M., Nizet, V., Beatson, S. A., Walker, M. J., and Sanderson-Smith, M. L. (2013) Streptococcal collagen-like protein $A$ and general stress protein 24 are immunomodulating virulence factors of group A Streptococcus. FASEB J. 27, 2633-2643

23. Cole, J. N., Ramirez, R. D., Currie, B. J., Cordwell, S. J., Djordjevic, S. P., and Walker, M. J. (2005) Surface analyses and immune reactivities of major cell wall-associated proteins of group A Streptococcus. Infect. Immun. 73, 3137-3146 
24. Stevens, D. L., Tanner, M. H., and Winship, J. (1989) Reappearance of scarlet fever toxin A among streptococci in the Rocky Mountain West: severe group A streptococcal infections associated with a toxic shock-like syndrome. N. Engl. J. Med. 321, 1-7

25. Musser, J. M., Hauser, A. R., Kim, M. H., Schlievert, P. M., Nelson, K., and Selander, R. K. (1991) Streptococcus pyogenes causing toxic-shock-like syndrome and other invasive diseases: clonal diversity and pyrogenic exotoxin expression. Proc. Natl. Acad. Sci. U. S. A. 88, 2668-2672

26. Cleary, P. P., Kaplan, E. L., Handley, J. P., Wlazlo, A., Kim, M. H., Hauser, A. R., and Schlievert, P. M. (1992) Clonal basis for resurgence of serious Streptococcus pyogenes disease in the 1980s. Lancet 339, 518-521

27. Bachert, B. A., Choi, S. J., LaSala, P. R., Harper, T. I., McNitt, D. H., Boehm, D. T., Caswell, C. C., Ciborowski, P., Keene, D. R., Flores, A. R., Musser, J. M., Squeglia, F., Marasco, D., Berisio, R., and Lukomski, S. (2016) Unique footprint in the sc/1.3 locus affects adhesion and biofilm formation of the invasive M3-type group A Streptococcus. Front Cell Infect Microbio/ 6, 90

28. Lukomski, S., Bachert, B. A., Squeglia, F., and Berisio, R. (2017) Collagen-like proteins of pathogenic streptococci. Mol. Microbiol. 103, 919-930

29. Xu, Y., Keene, D. R., Bujnicki, J. M., Höök, M., and Lukomski, S. (2002) Streptococcal Scl1 and Scl2 proteins form collagen-like triple helices. J Biol Chem 277, 27312-27318

30. Mohs, A., Silva, T., Yoshida, T., Amin, R., Lukomski, S., Inouye, M., and Brodsky, B. (2007) Mechanism of stabilization of a bacterial collagen triple helix in the absence of hydroxyproline. $J$ Biol Chem 282, 29757-29765

31. Okuyama, K. (2008) Revisiting the molecular structure of collagen. Connect. Tissue Res. 49, 299310

32. Brodsky, B., and Persikov, A. V. (2005) Molecular structure of the collagen triple helix. Adv. Protein Chem. 70, 301-339

33. Shoulders, M. D., and Raines, R. T. (2009) Collagen structure and stability. Annu. Rev. Biochem. 78, 929-958

34. Berisio, R., Vitagliano, L., Mazzarella, L., and Zagari, A. (2002) Crystal structure of the collagen triple helix model [(Pro-Pro-Gly) $\left.{ }_{10}\right]_{3}$. Protein Sci. 11, 262-270

35. Berisio, R., Granata, V., Vitagliano, L., and Zagari, A. (2004) Imino acids and collagen triple helix stability: characterization of collagen-like polypeptides containing Hyp-Hyp-Gly sequence repeats. J. Am. Chem. Soc. 126, 11402-11403

36. Berisio, R., Granata, V., Vitagliano, L., and Zagari, A. (2004) Characterization of collagen-like heterotrimers: Implications for triple-helix stability. Biopolymers 73, 682-688

37. Vitagliano, L., Berisio, R., Mastrangelo, A., Mazzarella, L., and Zagari, A. (2001) Preferred proline puckerings in cis and trans peptide groups: Implications for collagen stability. Protein Sci. 10, 2627-2632 
38. Berisio, R., De Simone, A., Ruggiero, A., Improta, R., and Vitagliano, L. (2009) Role of side chains in collagen triple helix stabilization and partner recognition. J. Pept. Sci. 15, 131-140

39. Improta, R., Berisio, R., and Vitagliano, L. (2008) Contribution of dipole-dipole interactions to the stability of the collagen triple helix. Protein Sci. 17, 955-961

40. Chan, V. C., Ramshaw, J. A., Kirkpatrick, A., Beck, K., and Brodsky, B. (1997) Positional preferences of ionizable residues in Gly-X-Y triplets of the collagen triple-helix. J Biol Chem 272, 31441-31446

41. Leikina, E., Mertts, M. V., Kuznetsova, N., and Leikin, S. (2002) Type I collagen is thermally unstable at body temperature. Proc. Natl. Acad. Sci. U. S. A. 99, 1314-1318

42. Han, R., Zwiefka, A., Caswell, C. C., Xu, Y., Keene, D. R., Lukomska, E., Zhao, Z., Höök, M., and Lukomski, S. (2006) Assessment of prokaryotic collagen-like sequences derived from streptococcal Scl1 and Scl2 proteins as a source of recombinant GXY polymers. Appl. Microbiol. Biotechnol. 72, 109-115

43. Xu, C., Yu, Z., Inouye, M., Brodsky, B., and Mirochnitchenko, O. (2010) Expanding the family of collagen proteins: recombinant bacterial collagens of varying composition form triple-helices of similar stability. Biomacromolecules 11, 348-356

44. Han, R., Caswell, C. C., Lukomska, E., Keene, D. R., Pawlowski, M., Bujnicki, J. M., Kim, J. K., and Lukomski, S. (2006) Binding of the low-density lipoprotein by streptococcal collagen-like protein Scl1 of Streptococcus pyogenes. Mol. Microbiol. 61, 351-367

45. Squeglia, F., Bachert, B., Romano, M., Lukomski, S., and Berisio, R. (2013) Crystallization and preliminary X-ray crystallographic analysis of the variable domain of Scl2.3, a streptococcal collagen-like protein from invasive M3-type Streptococcus pyogenes. Acta Crystallogr. Sect. F Struct. Biol. Cryst. Commun. 69, 1023-1025

46. Squeglia, F., Bachert, B., De Simone, A., Lukomski, S., and Berisio, R. (2014) The crystal structure of the streptococcal collagen-like protein 2 globular domain from invasive M3-type group A Streptococcus shows significant similarity to immunomodulatory HIV protein gp41. J Biol Chem 289, 5122-5133

47. Yu, Z., Mirochnitchenko, O., Xu, C., Yoshizumi, A., Brodsky, B., and Inouye, M. (2010) Noncollagenous region of the streptococcal collagen-like protein is a trimerization domain that supports refolding of adjacent homologous and heterologous collagenous domains. Protein Sci. 19, $775-785$

48. Humtsoe, J. O., Kim, J. K., Xu, Y., Keene, D. R., Höök, M., Lukomski, S., and Wary, K. K. (2005) A streptococcal collagen-like protein interacts with the $\alpha 2 \beta 1$ integrin and induces intracellular signaling. J. Biol. Chem. 280, 13848-13857

49. Caswell, C. C., Lukomska, E., Seo, N. S., Höök, M., and Lukomski, S. (2007) Scl1-dependent internalization of group A Streptococcus via direct interactions with the $\alpha 2 \beta 1$ integrin enhances pathogen survival and re-emergence. Mol. Microbiol. 64, 1319-1331 
50. Caswell, C. C., Barczyk, M., Keene, D. R., Lukomska, E., Gullberg, D. E., and Lukomski, S. (2008) Identification of the first prokaryotic collagen sequence motif that mediates binding to human collagen receptors, integrins $\alpha 2 \beta 1$ and $\alpha 11 \beta 1$. J Biol Chem 283, 36168-36175

51. Caswell, C. C., Han, R., Hovis, K. M., Ciborowski, P., Keene, D. R., Marconi, R. T., and Lukomski, S. (2008) The Scl1 protein of M6-type group A Streptococcus binds the human complement regulatory protein, factor $\mathrm{H}$, and inhibits the alternative pathway of complement. Mol. Microbiol. 67, 584-596

52. Caswell, C. C., Oliver-Kozup, H., Han, R., Lukomska, E., and Lukomski, S. (2010) Scl1, the multifunctional adhesin of group A Streptococcus, selectively binds cellular fibronectin and laminin, and mediates pathogen internalization by human cells. FEMS Microbiol. Lett. 303, 61-68

53. Reuter, M., Caswell, C. C., Lukomski, S., and Zipfel, P. F. (2010) Binding of the human complement regulators CFHR1 and factor $\mathrm{H}$ by streptococcal collagen-like protein 1 (Scl1) via their conserved $\mathrm{C}$ termini allows control of the complement cascade at multiple levels. $J$ Biol Chem 285, 38473-38485

54. Gao, Y., Liang, C., Zhao, R., Lukomski, S., and Han, R. (2010) The Scl1 of M41-type group A Streptococcus binds the high-density lipoprotein. FEMS Microbiol. Lett. 309, 55-61

55. Påhlman, L. I., Marx, P. F., Mörgelin, M., Lukomski, S., Meijers, J. C., and Herwald, H. (2007) Thrombin-activatable fibrinolysis inhibitor binds to Streptococcus pyogenes by interacting with collagen-like proteins A and B. J Biol Chem 282, 24873-24881

56. Dohrmann, S., Anik, S., Olson, J., Anderson, E. L., Etesami, N., No, H., Snipper, J., Nizet, V., and Okumura, C. Y. (2014) Role for streptococcal collagen-like protein 1 in M1T1 group A Streptococcus resistance to neutrophil extracellular traps. Infect. Immun. 82, 4011-4020

57. Oliver-Kozup, H. A., Elliott, M., Bachert, B. A., Martin, K. H., Reid, S. D., Schwegler-Berry, D. E., Green, B. J., and Lukomski, S. (2011) The streptococcal collagen-like protein-1 (Scl1) is a significant determinant for biofilm formation by group A Streptococcus. BMC Microbiol. 11, 262

58. Oliver-Kozup, H., Martin, K. H., Schwegler-Berry, D., Green, B. J., Betts, C., Shinde, A. V., Van De Water, L., and Lukomski, S. (2013) The group A streptococcal collagen-like protein-1, Scl1, mediates biofilm formation by targeting the extra domain A-containing variant of cellular fibronectin expressed in wounded tissue. Mol. Microbiol. 87, 672-689

59. Van De Water, L., Varney, S., and Tomasek, J. J. (2013) Mechanoregulation of the myofibroblast in wound contraction, scarring, and fibrosis: opportunities for new therapeutic intervention. Adv Wound Care (New Rochelle) 2, 122-141

60. Gurtner, G. C., Werner, S., Barrandon, Y., and Longaker, M. T. (2008) Wound repair and regeneration. Nature 453, 314-321

61. Clark, R. A., Lanigan, J. M., DellaPelle, P., Manseau, E., Dvorak, H. F., and Colvin, R. B. (1982) Fibronectin and fibrin provide a provisional matrix for epidermal cell migration during wound reepithelialization. J. Invest. Dermatol. 79, 264-269 
62. Olczyk, P., Mencner, Ł., and Komosinska-Vassev, K. (2014) The role of the extracellular matrix components in cutaneous wound healing. BioMed Research International 2014, 747584

63. Xue, M., and Jackson, C. J. (2015) Extracellular matrix reorganization during wound healing and its impact on abnormal scarring. Adv. Wound Care 4, 119-136

64. Pankov, R., and Yamada, K. M. (2002) Fibronectin at a glance. J. Cell Sci. 115, 3861-3863

65. Ffrench-Constant, C. (1995) Alternative splicing of fibronectin--many different proteins but few different functions. Exp. Cell Res. 221, 261-271

66. Hynes, R. O. (1990) Fibronectins, Springer-Verlag, New York, NY

67. To, W. S., and Midwood, K. S. (2011) Plasma and cellular fibronectin: distinct and independent functions during tissue repair. Fibrogenesis Tissue Repair 4, 21

68. Ffrench-Constant, C., and Hynes, R. O. (1989) Alternative splicing of fibronectin is temporally and spatially regulated in the chicken embryo. Development 106, 375-388

69. Ffrench-Constant, C., Van De Water, L., Dvorak, H. F., and Hynes, R. O. (1989) Reappearance of an embryonic pattern of fibronectin splicing during wound healing in the adult rat. J. Cell Biol. 109, 903-914

70. Coito, A. J., Brown, L. F., Peters, J. H., Kupiec-Weglinski, J. W., and Van De Water, L. (1997) Expression of fibronectin splicing variants in organ transplantation: a differential pattern between rat cardiac allografts and isografts. Am. J. Pathol. 150, 1757-1772

71. Singh, P., Reimer, C. L., Peters, J. H., Stepp, M. A., Hynes, R. O., and Van De Water, L. (2004) The spatial and temporal expression patterns of integrin $\alpha 9 \beta 1$ and one of its ligands, the EIIIA segment of fibronectin, in cutaneous wound healing. J. Invest. Dermatol. 123, 1176-1181

72. Muro, A. F., Chauhan, A. K., Gajovic, S., laconcig, A., Porro, F., Stanta, G., and Baralle, F. E. (2003) Regulated splicing of the fibronectin EDA exon is essential for proper skin wound healing and normal lifespan. J. Cell Biol. 162, 149-160

73. Longmate, W. M., Lyons, S. P., Chittur, S. V., Pumiglia, K. M., Van De Water, L., and DiPersio, C. M. (2017) Suppression of integrin $\alpha 3 \beta 1$ by $\alpha 9 \beta 1$ in the epidermis controls the paracrine resolution of wound angiogenesis. J. Cell Biol. 216, 1473-1488

74. Leahy, D. J., Aukhil, I., and Erickson, H. P. (1996) 2.0 A crystal structure of a four-domain segment of human fibronectin encompassing the RGD loop and synergy region. Cell 84, 155-164

75. Niimi, T., Osawa, M., Yamaji, N., Yasunaga, K., Sakashita, H., Mase, T., Tanaka, A., and Fujita, S. (2001) NMR structure of human fibronectin EDA. J. Biomol. NMR 21, 281-284

76. Tan, M. H., Sun, Z., Opitz, S. L., Schmidt, T. E., Peters, J. H., and George, E. L. (2004) Deletion of the alternatively spliced fibronectin EIIIA domain in mice reduces atherosclerosis. Blood 104, $11-18$

77. Muro, A. F., Moretti, F. A., Moore, B. B., Yan, M., Atrasz, R. G., Wilke, C. A., Flaherty, K. R., Martinez, F. J., Tsui, J. L., Sheppard, D., Baralle, F. E., Toews, G. B., and White, E. S. (2008) An 
essential role for fibronectin extra type III domain A in pulmonary fibrosis. Am. J. Respir. Crit. Care Med. 177, 638-645

78. Babaev, V. R., Porro, F., Linton, M. F., Fazio, S., Baralle, F. E., and Muro, A. F. (2008) Absence of regulated splicing of fibronectin EDA exon reduces atherosclerosis in mice. Atherosclerosis 197, 534-540

79. Chauhan Anil, K., Kisucka, J., Cozzi Maria, R., Walsh Meghan, T., Moretti Federico, A., Battiston, M., Mazzucato, M., De Marco, L., Baralle Francisco, E., Wagner Denisa, D., and Muro Andrés, F. (2008) Prothrombotic Effects of Fibronectin Isoforms Containing the EDA Domain. Arterioscler.

Thromb. Vasc. Biol. 28, 296-301

80. Chauhan, A. K., Moretti, F. A., laconcig, A., Baralle, F. E., and Muro, A. F. (2005) Impaired motor coordination in mice lacking the EDA exon of the fibronectin gene. Behav. Brain Res. 161, 31-38

81. Liao, Y. F., Gotwals, P. J., Koteliansky, V. E., Sheppard, D., and Van De Water, L. (2002) The EIIIA segment of fibronectin is a ligand for integrins $\alpha 9 \beta 1$ and $\alpha 4 \beta 1$ providing a novel mechanism for regulating cell adhesion by alternative splicing. J Biol Chem 277, 14467-14474

82. Shinde, A. V., Bystroff, C., Wang, C., Vogelezang, M. G., Vincent, P. A., Hynes, R. O., and Van De Water, L. (2008) Identification of the peptide sequences within the EIIIA (EDA) segment of fibronectin that mediate integrin a9b1-dependent cellular activities. J Biol Chem 283, 2858-2870

83. Kohan, M., Muro, A. F., White, E. S., and Berkman, N. (2010) EDA-containing cellular fibronectin induces fibroblast differentiation through binding to a4ß37 integrin receptor and MAPK/Erk 1/2dependent signaling. FASEB J. 24, 4503-4512

84. White, E. S., and Muro, A. F. (2011) Fibronectin splice variants: understanding their multiple roles in health and disease using engineered mouse models. IUBMB Life 63, 538-546

85. Singh, P., Chen, C., Pal-Ghosh, S., Stepp, M. A., Sheppard, D., and Van De Water, L. (2009) Loss of integrin $\alpha 9 \beta 1$ results in defects in proliferation, causing poor re-epithelialization during cutaneous wound healing. J. Invest. Dermatol. 129, 217-228

86. Shinde, A. V., Kelsh, R., Peters, J. H., Sekiguchi, K., Van De Water, L., and McKeown-Longo, P. J. (2015) The $\alpha 4 \beta 1$ integrin and the EDA domain of fibronectin regulate a profibrotic pheotype in dermal fibroblasts Matrix Biol. 41, 26-35

87. Zardi, L., Carnemolla, B., Siri, A., Petersen, T. E., Paolella, G., Sebastio, G., and Baralle, F. E. (1987) Transformed human cells produce a new fibronectin isoform by preferential alternative splicing of a previously unobserved exon. EMBO J. 6, 2337-2342

88. Kraft, S., Klemis, V., Sens, C., Lenhard, T., Jacobi, C., Samstag, Y., Wabnitz, G., Kirschfink, M., Wallich, R., Hänsch, G. M., and Nakchbandi, I. A. (2016) Identification and characterization of a unique role for EDB fibronectin in phagocytosis. J. Mol. Med. 94, 567-581

89. Castellani, P., Viale, G., Dorcaratto, A., Nicolo, G., Kaczmarek, J., Querze, G., and Zardi, L. (1994) The fibronectin isoform containing the ED-B oncofetal domain: A marker of angiogenesis. Int. J. Cancer 59, 612-618 
90. Fukuda, T., Yoshida, N., Kataoka, Y., Manabe, R.-i., Mizuno-Horikawa, Y., Sato, M., Kuriyama, K., Yasui, N., and Sekiguchi, K. (2002) Mice lacking the EDB segment of fibronectin develop normally but exhibit reduced cell growth and fibronectin matrix assembly in vitro. Cancer Res. 62, 5603-5610

91. Astrof, S., Crowley, D., George, E. L., Fukuda, T., Sekiguchi, K., Hanahan, D., and Hynes, R. O. (2004) Direct test of potential roles of EIIIA and EIIIB alternatively spliced segments of fibronectin in physiological and tumor angiogenesis. Mol. Cell. Biol. 24, 8662-8670

92. Matuskova, J., Chauhan, A. K., Cambien, B., Astrof, S., Dole, V. S., Piffath, C. L., Hynes, R. O., and Wagner, D. D. (2006) Decreased plasma fibronectin leads to delayed thrombus growth in injured arterioles. Arterioscler. Thromb. Vasc. Biol. 26, 1391-1396

93. Astrof, S., Crowley, D., and Hynes, R. O. (2007) Multiple cardiovascular defects caused by the absence of alternatively spliced segments of fibronectin. Dev. Biol. 311, 11-24

94. Chen, S., Chakrabarti, R., Keats, E. C., Chen, M., Chakrabarti, S., and Khan, Z. A. (2012) Regulation of Vascular Endothelial Growth Factor Expression by Extra Domain B Segment of Fibronectin in Endothelial CellsVEGF Expression by Fibronectin. Invest. Ophthalmol. Vis. Sci. 53, 8333-8343

95. Schiefner, A., Gebauer, M., and Skerra, A. (2012) Extra-domain B in oncofetal fibronectin structurally promotes fibrillar head-to-tail dimerization of extracellular matrix protein. J. Biol. Chem. 287, 17578-17588

96. Midwood, K. S., and Orend, G. (2009) The role of tenascin-C in tissue injury and tumorigenesis. $J$ Cell Commun Signal 3, 287-310

97. Giblin, S. P., and Midwood, K. S. (2015) Tenascin-C: Form versus function. Cell Adh. Migr. 9, 4882

98. Gulcher, J. R., Nies, D. E., Alexakos, M. J., Ravikant, N. A., Sturgill, M. E., Marton, L. S., and Stefansson, K. (1991) Structure of the human hexabrachion (tenascin) gene. Proc. Natl. Acad. Sci. U. S. A. 88, 9438-9442

99. Gulcher, J. R., Nies, D. E., Marton, L. S., and Stefansson, K. (1989) An alternatively spliced region of the human hexabrachion contains a repeat of potential $\mathrm{N}$-glycosylation sites. Proc. Natl. Acad. Sci. U. S. A. 86, 1588-1592

100. Adams, J. C., Chiquet-Ehrismann, R., and Tucker, R. P. (2015) The evolution of tenascins and fibronectin. Cell Adh. Migr. 9, 22-33

101. Carr, P. A., Erickson, H. P., and Palmer, A. G., III. (1997) Backbone dynamics of homologous fibronectin type III cell adhesion domains from fibronectin and tenascin. Structure 5, 949-959

102. Page, T. H., Charles, P. J., Piccinini, A. M., Nicolaidou, V., Taylor, P. C., and Midwood, K. S. (2012) Raised circulating tenascin-C in rheumatoid arthritis. Arthritis Res. Ther. 14, R260-R260 
103. Whitby, D. J., Longaker, M. T., Harrison, M. R., Adzick, N. S., and Ferguson, M. W. (1991) Rapid epithelialisation of fetal wounds is associated with the early deposition of tenascin. J. Cell Sci. 99 (Pt 3), 583-586

104. Kusubata, M., Hirota, A., Ebihara, T., Kuwaba, K., Matsubara, Y., Sasaki, T., Kusakabe, M., Tsukada, T., Irie, S., and Koyama, Y. (1999) Spatiotemporal changes of fibronectin, tenascin-C, fibulin-1, and fibulin-2 in the skin during the development of chronic contact dermatitis. J. Invest. Dermatol. 113, 906-912

105. Betz, P., Nerlich, A., Tübel, J., Penning, R., and Eisenmengen, W. (1993) Localization of tenascin in human skin wounds -An immunohistochemical study. Int. J. Legal Med. 105, 325-328

106. Chiquet-Ehrismann, R., Tannheimer, M., Koch, M., Brunner, A., Spring, J., Martin, D., Baumgartner, S., and Chiquet, M. (1994) Tenascin-C expression by fibroblasts is elevated in stressed collagen gels. J. Cell Biol. 127, 2093-2101

107. Latijnhouwers, M., Bergers, M., Ponec, M., Dijkman, H., Andriessen, M., and Schalkwijk, J. (1997) Human Epidermal Keratinocytes Are a Source of Tenascin-C during Wound Healing. J. Invest. Dermatol. 108, 776-783

108. Latijnhouwers, M. A., Bergers, M., Van Bergen, B. H., Spruijt, K. I., Andriessen, M. P., and Schalkwijk, J. (1996) Tenascin expression during wound healing in human skin. J. Pathol. 178, 30-35

109. Chiquet-Ehrismann, R., Mackie, E. J., Pearson, C. A., and Sakakura, T. (1986) Tenascin: an extracellular matrix protein involved in tissue interactions during fetal development and oncogenesis. Cell 47, 131-139

110. Huang, W., Chiquet-Ehrismann, R., Moyano, J. V., Garcia-Pardo, A., and Orend, G. (2001) Interference of tenascin- $C$ with syndecan- 4 binding to fibronectin blocks cell adhesion and stimulates tumor cell proliferation. Cancer Res. 61, 8586-8594

111. Midwood, K. S., Valenick, L. V., Hsia, H. C., and Schwarzbauer, J. E. (2004) Coregulation of Fibronectin Signaling and Matrix Contraction by Tenascin-C and Syndecan-4. Mol. Biol. Cell 15, 5670-5677

112. Tucker, R. P., and Chiquet-Ehrismann, R. (2015) Tenascin-C: Its functions as an integrin ligand. Int. J. Biochem. Cell Biol. 65, 165-168

113. Yokosaki, Y., Palmer, E. L., Prieto, A. L., Crossin, K. L., Bourdon, M. A., Pytela, R., and Sheppard, D. (1994) The integrin a9ß1 mediates cell attachment to a non-RGD site in the third fibronectin type III repeat of tenascin. J. Biol. Chem. 269, 26691-26696

114. Yokosaki, Y., Matsuura, N., Higashiyama, S., Murakami, I., Obara, M., Yamakido, M., Shigeto, N., Chen, J., and Sheppard, D. (1998) Identification of the ligand binding site for the integrin $\alpha 9 \beta 1$ in the third fibronectin type III repeat of tenascin-C. J Biol Chem 273, 11423-11428

115. Yoshida, T., Akatsuka, T., and Imanaka-Yoshida, K. (2015) Tenascin-C and integrins in cancer. Cell Adh Migr 9, 96-104 
116. Katz, S., Hukkanen, M., Lounatmaa, K., Rousselle, P., Tervo, T., and Virtanen, I. (2006) Cooperation of isoforms of laminin-332 and tenascin-CL during early adhesion and spreading of immortalized human corneal epithelial cells. Exp. Eye Res. 83, 1412-1422

117. End, P., Panayotou, G., Entwistle, A., Waterfield, M. D., and Chiquet, M. (1992) Tenascin: a modulator of cell growth. Eur. J. Biochem. 209, 1041-1051

118. Day, J. M., Olin, A. I., Murdoch, A. D., Canfield, A., Sasaki, T., Timpl, R., Hardingham, T. E., and Aspberg, A. (2004) Alternative splicing in the aggrecan G3 domain influences binding interactions with tenascin-C and other extracellular matrix proteins. J Biol Chem 279, 12511-12518

119. Tamaoki, M., Imanaka-Yoshida, K., Yokoyama, K., Nishioka, T., Inada, H., Hiroe, M., Sakakura, T., and Yoshida, T. (2005) Tenascin-C regulates recruitment of myofibroblasts during tissue repair after myocardial injury. Am. J. Pathol. 167, 71-80

120. Frick, I. M., Crossin, K. L., Edelman, G. M., and Björck, L. (1995) Protein H-a bacterial surface protein with affinity for both immunoglobulin and fibronectin type III domains. EMBO J.14, 16741679

121. Herrera, A. L., Faal, H., Moss, D., Addengast, L., Fanta, L., Eyster, K., Huber, V. C., and Chaussee, M. S. (2018) The Streptococcus pyogenes fibronectin/tenascin-binding protein PrtF.2 contributes to virulence in an influenza superinfection. Sci. Rep. 8, 12126

122. Henderson, B., Nair, S., Pallas, J., and Williams, M. A. (2011) Fibronectin: a multidomain host adhesin targeted by bacterial fibronectin-binding proteins. FEMS Microbiol. Rev. 35, 147-200

123. Paulsson, M., and Riesbeck, K. (2018) How bacteria hack the matrix and dodge the bullets of immunity. Eur. Respir. Rev. 27

124. Signäs, C., Raucci, G., Jönsson, K., Lindgren, P. E., Anantharamaiah, G. M., Höök, M., and Lindberg, M. (1989) Nucleotide sequence of the gene for a fibronectin-binding protein from Staphylococcus aureus: use of this peptide sequence in the synthesis of biologically active peptides. Proc. Natl. Acad. Sci. U. S. A. 86, 699-703

125. Schwarz-Linek, U., Werner, J. M., Pickford, A. R., Gurusiddappa, S., Kim, J. H., Pilka, E. S., Briggs, J. A., Gough, T. S., Höök, M., Campbell, I. D., and Potts, J. R. (2003) Pathogenic bacteria attach to human fibronectin through a tandem beta-zipper. Nature 423, 177-181

126. Talay, S. R., Valentin-Weigand, P., Jerlstrom, P. G., Timmis, K. N., and Chhatwal, G. S. (1992) Fibronectin-binding protein of Streptococcus pyogenes: sequence of the binding domain involved in adherence of streptococci to epithelial cells. Infect. Immun. 60, 3837-3844

127. Jaffe, J., Natanson-Yaron, S., Caparon, M. G., and Hanski, E. (1996) Protein F2, a novel fibronectin-binding protein from Streptococcus pyogenes, possesses two domains. Mol. Microbiol. 21, 373-384

128. Rakonjac, J. V., Robbins, J. C., and Fischetti, V. A. (1995) DNA sequence of the serum opacity factor of group A streptococci: identification of a fibronectin-binding repeat domain. Infect. Immun. 63, 622-631 
129. Terao, Y., Kawabata, S., Kunitomo, E., Murakami, J., Nakagawa, I., and Hamada, S. (2001) Fba, a novel fibronectin-binding protein from Streptococcus pyogenes, promotes bacterial entry into epithelial cells, and the fba gene is positively transcribed under the Mga regulator. Mol. Microbiol.

42, 75-86

130. House-Pompeo, K., Xu, Y., Joh, D., Speziale, P., and Höök, M. (1996) Conformational changes in the fibronectin binding MSCRAMMs are induced by ligand binding. J Biol Chem 271, 1379-1384

131. Talay, S. R., Zock, A., Rohde, M., Molinari, G., Oggioni, M., Pozzi, G., Guzman, C. A., and Chhatwal, G. S. (2000) Co-operative binding of human fibronectin to Sfbl protein triggers streptococcal invasion into respiratory epithelial cells. Cell. Microbiol. 2, 521-535

132. Ozeri, V., Rosenshine, I., Mosher, D. F., Fässler, R., and Hanski, E. (1998) Roles of integrins and fibronectin in the entry of Streptococcus pyogenes into cells via protein F1. Mol. Microbiol. $\mathbf{3 0}$, 625-637

133. Terao, Y., Kawabata, S., Nakata, M., Nakagawa, I., and Hamada, S. (2002) Molecular characterization of a novel fibronectin-binding protein of Streptococcus pyogenes strains isolated from toxic shock-like syndrome patients. J Biol Chem 277, 47428-47435

134. Fisher, M., Huang, Y.-S., Li, X., Mclver, K. S., Toukoki, C., and Eichenbaum, Z. (2008) Shr is a broad-spectrum surface receptor that contributes to adherence and virulence in group $A$ Streptococcus. Infect. Immun. 76, 5006-5015 


\title{
CHAPTER 1
}

\section{SURFACE-EXPOSED LOOPS AND AN ACIDIC PATCH IN THE SCL1 PROTEIN OF GROUP}

\section{A STREPTOCOCCUS ENABLE SCL1 BINDING TO WOUND-ASSOCIATED FIBRONECTIN}

Dudley H. McNitt, Soo Jeon Choi, Douglas R. Keene, Livingston Van De Water, Flavia

Squeglia, Rita Berisio, Slawomir Lukomski

Published in The Journal of Biological Chemistry 2018 293(20): 7796-7810.

\begin{abstract}
Keratinized epidermis constitutes a powerful barrier of the mucosa and skin, effectively preventing bacterial invasion, unless it is wounded and no longer protective. Wound healing involves deposition of distinct extracellular matrix (ECM) proteins enriched in cellular fibronectin (cFn) isoforms containing extra domain A (EDA). The streptococcal collagen-like protein 1 (Scl1) is a surface adhesin of group A Streptococcus (GAS), which contains an $\mathrm{N}$-terminal variable (V) domain and a C-terminally located collagen-like domain. During wound infection, Scl1 selectively binds EDA/cFn isoforms and laminin, as well as low-density lipoprotein ( $L D L)$, through its $V$ domain. The trimeric $V$ domain has a six-helical bundle fold composed of three pairs of anti-parallel $\alpha$-helices interconnected by hypervariable loops, but the roles of these structures in EDA/cFn binding are unclear. Here, using recombinant $\mathrm{Scl}$ constructs $(\mathrm{rScl})$ to investigate structure-function determinants of the Scl1-EDA/cFn interaction, we found that full-length rScl1, containing both the globular $\mathrm{V}$ and the collagen domains, is necessary for EDA/cFn binding. We established that the surface-exposed loops, inter-connecting conserved $\alpha$-helices, guide recognition and binding of Scl1-V to EDA and binding to laminin and LDL. Moreover, electrostatic surface potential models of the Scl1 V domains pointed to a conserved, negatively charged pocket, surrounded by positively charged and neutral regions, as a determining factor for the binding. In light of these findings, we propose an updated model of EDA/cFn recognition by the Scl1 adhesin from GAS, representing a significant step in understanding the Scl1-ECM interactions within the wound microenvironment that underlie GAS pathogenesis.
\end{abstract}




\section{INTRODUCTION}

Group A Streptococcus (GAS) is a human adapted pathogen responsible for over 700 million disease-associated infections across the globe each year and an estimated healthcare cost of 224-539 million dollars in the United States alone $(1,2)$. GAS asymptomatically colonizes the general population of adults (5-15\%) and children (20$30 \%$ ), most of the morbidity is associated with superficial infections of the throat and skin (3). In addition, bacterial spread may result in severe soft tissue and systemic infections, like necrotizing fasciitis and streptococcal toxic shock syndrome (4), or may lead to postinfectious sequalae, such as acute rheumatic fever and rheumatic heart disease, poststreptococcal glomerulonephritis, and pediatric autoimmune neuropsychiatric disorders associated with streptococcal infections $(5,6)$. For the initial colonization of the human host, GAS requires a portal of entry, which is constituted by a breach in the epithelial cell lining (thenceforth as wound) that allows the pathogen to spread into deeper tissue and blood. GAS expresses numerous surface adhesins that bind to host extracellular matrix (ECM) (7). ECM binding facilitates initial localized colonization, during which GAS forms tissue microcolonies embedded in glycocalyx (8). The interplay between GAS adhesins and host's ECM-components, stable tissue microcolony formation, and superficial versus invasive infection are of significant clinical interest.

Among prominent GAS surface proteins are the streptococcal collagen-like (Scl) proteins 1 and 2 (9-13). Scl1 and Scl2 are homotrimeric proteins that have similar domain organization, which is comprised of an $\mathrm{N}$-terminal globular sequence-variable $(\mathrm{V})$ domain, a collagen-like $(C L)$ domain, and the C-terminal cell-wall anchoring domain $(9,10)$. The CL-domain of both Scl1 and Scl2 consists of varying numbers of Gly-Xaa-Yaa collagen repeats that adopt a right-handed triple-helix structure, similar to mammalian collagen (14-16); Scl1-CL domain may interact with human collagen-binding integrins, promoting GAS internalization (17-19). The outermost-exposed $V$ domains vary in amino acid sequence between and within Scl1 and Scl2 variants and are typically associated with $\mathrm{M}$ type antigen $(9,11)$. Despite significant sequence variation, the $\mathrm{V}$ domains of Scl1 and Scl2 were predicted to contain two conserved $\alpha$-helices, spaced by hypervariable regions $(11,20)$. Recent reports of a first crystal structure for the Scl2-V domain validated these secondary structure predictions $(21,22)$. The recombinant Scl2-V globular domain forms 
a trimeric structure, which folds into a 6-helix bundle, with 3 pairs of anti-parallel $\alpha$-helices inter-connected by hypervariable loops (22). Despite both Scl1 and Scl2 having similar structural organization, they play different roles in GAS pathogenesis (23). While the number of Scl2 targets identified remains scanty (24), Scl1 variants from diverse GAS M types bind several host proteins. Ligands include tissue components, cellular fibronectin (cFn) and laminin (Lm), as well as blood components, low- and high-density lipoproteins, and complement Factor $\mathrm{H}$ (CFH) and Factor H-related protein 1 (CFHR1), (20,25-28). It is not clear how the variable sequences of the Scl1-V domain bind the same ligands, but two mutually-exclusive binding patterns were observed. Scl1 proteins from several Mtypes bind to ECM proteins, cFn and Lm, and low-density lipoprotein (LDL), but not FH and CFHR1; the converse is also true.

One of the major ECM targets for GAS within wounded tissue is fibronectin (Fn) (7). Fn is a high molecular weight glycoprotein that is found throughout the human body in two main forms, either as a soluble circulating plasma fibronectin $(\mathrm{pFn})$ secreted by hepatocytes or as an insoluble cellular fibronectin (cFn) secreted by a variety of cells within tissues (29). All forms of Fn are transcribed from a single gene, FN1, and are comprised of three modules of fibronectin repeats: type I, II, and III $(29,30)$. There are about 20 isoforms of human cFn that include combinations of the type III repeats, e.g. extra domain $A(E D A / E I I I A)$ and $B(E D B / E I I I B)$, and a varied number of $V$ repeats $(30,31)$. These isoforms differ by spatial-temporal distribution following injury $(32,33)$. Specifically, the EDA-containing isoforms are normally expressed during embryo-genesis, though, they are found in negligible levels in most adult tissues, except in healing wounds where EDA/cFn is highly upregulated $(32,34)$. The EDA fold is a $\beta$-sandwich structure formed by seven antiparallel $\beta$-strands (i.e. A, B, C, C', D, E, and F) connected by loops $(35,36)$. The C-C' loop is an important site for cell attachment, via integrin receptors $\alpha_{9} \beta_{1}$ and $\alpha_{4} \beta_{1}$ $(37,38)$, and attachment to the C-C' loop by these integrins has been shown to regulate important tissue repair and wound healing functions (39-43).

Here, we established that surface-exposed loops within V domain structure guide Scl1 to bind EDA/cFn, as well as two other Scl1 ligands, laminin and apolipoprotein B100 (ApoB100) of LDL. A second conserved constituent within variable Scl1-V domain is a common topography of the electrostatic surface potential surrounding guiding loops, 
which includes a negatively-charged patch formed by collective Asp and Glu residues contributed by all three monomeric chains. The proposed model accommodates binding of common ligands by phylogenetically diverse Scl1 variants. This work implies the evolutionary response of the Scl1-V domain to selective pressure, resulting in the retention of the conserved structure and function of ligand binding. This underscores the importance of Scl1 binding to EDA-containing fibronectins deposited at the pathogen portal of entry.

\section{RESULTS}

\section{The globular sequence-variable (V) domains of Scl1 and Scl2 contain conserved} helix-loop-helix structures - Two streptococcal collagen-like proteins, Scl1 and Scl2, display a similar "lollipop-like" structural organization (Fig. 1A), which is comprised of an $\mathrm{N}$-terminal globular V-domain head and a C-terminal CL-domain stalk (14). Multiple sequence alignments illustrate a significant variations in the $\mathrm{V}$ regions, both between and within Scl1 and Scl2 families (Fig. 1B). Despite sequence variation, several secondary structure prediction algorithms deduced that both Scl1-V and Scl2-V domains contain two conserved $\alpha$-helices inter-connected by hypervariable regions. Crystallography data rendered for the recombinant Scl2-V construct, derived from M3-type GAS, revealed that rScl2.3-V protein forms a homotrimer, with three pairs of conserved $\alpha$-helices forming a 6-helix bundle and hypervariable regions forming surface-exposed loops (PDB accession code: $4 \mathrm{nsm}$ ). To examine the conservation of the helix-loop-helix motif, homology models of two sequence-diverse Scl1- and Scl2-V domains were generated (Fig. 1C), using the structure of Scl2.3-V domain as template. The homology model structure of Scl1.1-V domain from M1-type GAS was based on the consensus- sequence alignment (24\% sequence identity on 65 amino acid residues), and the model of Scl2.28-V domain from M28 GAS was obtained with the same approach (51.5\% identity with Scl2.3-V sequence on 74 residues). Homology models predict with high confidence that Scl1.1-V ( $E=3.5 x$ $\left.10^{-16}\right)$ and $\mathrm{Scl} 2.28-\mathrm{V}\left(\mathrm{E}=1.8 \times 10^{-40}\right)$ domains each adopts a similar six-helical-bundle trimeric structure, as Scl2.3-V template. The solved crystal structure and homology models rendered here show that globular domains of Scl1.1 and Scl2.28 variants can trimerize without the presence of the CL-domain, an observation that was also found in 
Scl2 proteins from other M-types (44). Furthermore, we hypothesized that trimeric rScl1$\mathrm{V}$ constructs will retain their binding function to ECM ligands.

\section{The conserved structure of Scl1-V domain requires the collagen domain for ligand} binding - Full-length rScl1 proteins, derived from different GAS M-types, bind to host cFn via interactions with EDA, which is mediated by Scl1-V domain $(25,45)$. Here, we examined whether the $\mathrm{V}$-domain-alone $\mathrm{rScl} 1$ constructs bind to $\mathrm{cFn}$ and rEDA. Full-length recombinant Scl1 constructs, derived from M1- (rScl1.1FL) and M3-type (rScl1.3FL) strains, as well as their corresponding V-domain-only proteins (rScl1.1V and rScl1.3V) were immobilized in wells and tested for ECM-ligand binding. Unexpectedly, neither rScl1.1V nor rScl1.3V constructs bound rEDA and cFn, while the corresponding full-length rScl1FL control proteins bound both ligands (Fig. 2). Thus, while the $\mathrm{V}$ domains of Scl1 proteins can fold independently of their $C L$ domains, there are currently unknown structural constraints that require the presence of continuous $C L$ domains for EDA/cFn recognition.

The loop region of Scl1-V domain plays a central role in ECM recognition and binding - The binding site for the Scl1-V domain within the loop segment located between C- and C'- $\beta$-strands of the EDA $\beta$-sandwich (45). Here, we hypothesized that EDA binds to surface-exposed loops of Scl1.1-V domain, which we since identified in the crystal structure and deduced in the homology models (Fig. 1C). To test this hypothesis, we generated two full-length chimeric rScl.chi proteins: (i) we inserted the 22-amino-acid loop sequence of the ECM-binding-positive rScl1.1 variant (residues 25-46 of the V-domain) in place of the respective loop sequence of the ECM-binding-negative protein rScl2.28 (residues 20-45), resulting in the chimeric construct rScl.chi1FL (Fig. 3A); and (ii) the reverse was also constructed, by inserting the 26-amino-acid-loop region of rScl2.28-V in place of loop sequence of $\mathrm{rScl1} 1 \mathrm{1-V}$, resulting in rScl.chi2FL. To identify the proper site for inserting these sequences, we considered secondary structure predictions, computed with JPRED; resulting models were then minimized using GROMACS.

To ensure that we did not substantially alter the overall structure of the chimeric proteins, both rScl.chi1FL and rScl.chi2FL were examined by circular dichroism (CD) 
spectroscopy and electron microscopy of rotary shadowed samples (Fig. $3 B$ ). The wavelength scans of the original $\mathrm{rScl} 1.1 \mathrm{FL}$ and $\mathrm{rScl} 2.28 \mathrm{FL}$ proteins each exhibit a positive peak of molar ellipticity at $220 \mathrm{~nm},[\theta]_{220}(14)$, which is a characteristics of the collagen triple helix (46). The CD spectra of both chimeric proteins, rScl.chi1FL and rScl.chi2FL, rendered at $20^{\circ} \mathrm{C}$, show peak ellipticity values at $220 \mathrm{~nm}$ (Fig. 3B, solid lines), thus, demonstrating that both chimeric constructs adopt triple helix structures. These peaks were not observed at $50^{\circ} \mathrm{C}$, indicating that each triple helix unfolded (Fig. 3B, dashed lines). The domain organization of both chimeric rScls was further examined via rotary shadowing and subsequent electron microscopy. This technique has been used previously by our lab and others to investigate the structure of multi-domain collagens, containing collagenous and non-collagenous domains $(14,47)$. Both chimeric rScls displayed the characteristic two-domain lollipop-like organization, with the CL domain, as the stalk, and the globular V-domain head (Fig. 3B). Thus, swapping the loop regions between rScl1.1FL and $\mathrm{rScl} 2.28 \mathrm{FL}$ did not alter the overall structural organization and triple-helices in the chimeric proteins.

We next examined the binding characteristics of both chimeric proteins to rEDA and $\mathrm{cFn}$ ligands, with $\mathrm{rScl} 1$.1FL and $\mathrm{rScl} 2.28 \mathrm{FL}$ serving as positive and negative controls, respectively. Binding data show that rScl.chi1FL chimera, containing donor-loop sequence of rScl1.1FL, binds both rEDA and cFn, while the reverse construct rScl.chi2FL did not bind to either ligand, similar to negative control protein rScl2.28FL (Fig. 3C). These data demonstrate the importance of the loop region in ECM binding for Scl1.1FL, and hypothetically for other Scl1 variants.

To assess the importance of the Scl1.1-V loop in rEDA recognition in the context of whole-GAS cells, we complemented in-trans the sc/1-inactivated mutant ( $\Delta s c / 1)$ of M1type strain (9) for the expression of original Scl1.1 $(\Delta s c / 1:: s c / 1.1)$ or chimeric Scl.chi2 protein ( $\Delta s c / 1:: s c l . c h i 2)$, using plasmids pSL620 and pSL621 (Table 1). The M1-type wild type (WT) and $\Delta s c / 1$ mutant strains were used as positive and negative controls, respectively (Fig. 3D, left panel). Expression of surface Scl1.1 and Scl.chi2 was assessed via western immunoblotting of the cell wall fractions. Additionally, rScl1.1FL protein was loaded, as a positive control (rScl1.1FL is smaller on the SDS gel since it is devoid of the cell-wall region of native Scl1.1 protein). Western blot analysis detected an 
immunoreactive band of $\sim 55 \mathrm{kDa}$, corresponding to the Scl1.1 surface protein (9), in WT and both complemented strains but not in $\Delta s c / 1$ mutant complemented with vector alone. It should be noted that lower amounts of protein samples, prepared from complemented strains ( $5 \mu \mathrm{Ls}$ versus $25 \mu \mathrm{Ls}$ of WT and $\Delta s c / 1$ mutant samples), were loaded on the SDS gel to avoid an overwhelming chemiluminescent signal. This is not surprising since Scl1.1FL and Scl.chi2FL proteins are expressed in complemented strains from multicopy plasmids. A similar expression pattern was recorded by flow cytometry of GAS cells. Approximately $66 \%$ of the WT cells had detectable levels of surface Scl1.1 and only $2 \%$ background level recorded for $\Delta s c / 1$ mutant, compared to $77 \%$ and $92 \%$ detection recorded for trans-complemented $\Delta s c / 1:: s c / 1.1$ and $\Delta s c / 1:: s c l . c h i 2$, respectively (not shown).

We next investigated the rEDA binding to whole GAS cells by flow cytometry (Fig. $3 D$, right panel). As expected, the WT and $\Delta s c / 1:: s c / 1.1$-complemented cells bind rEDA, although, the complemented strain binds $\sim 4$ times more ligand due to overexpression of the surface Scl1.1 protein. In contrast, neither $\Delta s c / 1$ mutant nor complemented GAS cells, overexpressing the Scl.ch2 protein, bind rEDA. Thus, GAS cells that express native Scl1 adhesin bind rEDA to the cell surface but lose this capacity once cells express a mutated variant with loop substitution from Scl2 protein, which does not bind EDA/cFn.

\section{The C-terminus of the loop region of Scl1-V domain plays a central role in ECM} recognition and binding - We next designed additional chimeric $\mathrm{rScl}$ constructs to identify the specific region of the loop involved in rEDA and cFn binding. Instead of replacing the entire 22 amino acids, the Scl1.1-V loop was substituted into Scl2.28-V loop in three segments (Fig. 4A). For the first construct, only the $\mathrm{N}$-terminal 11 amino acids of Scl1.1-V loop (residues 25-35) were substituted in place of the first 11 amino acids of the corresponding Scl2.28-V sequence (residues 20-30), resulting in construct rScl.chiNFL. Similarly, the C-terminal 11 amino acids of Scl1.1-V (residues 36-46) were substituted in place of the C-terminal 15 amino acids of Scl2.28 (residues 31-45), resulting in construct rScl.chiCFL. The third construct, rScl.chilFL, contains the internal 11 amino acids of Scl1.1-V (residues 30-40) in place of the central (residues 25-36) 11 amino acids of Scl2.28-V. 
All three chimeric rScls, containing partial loop substitutions, were examined by $C D$ spectroscopy and electron microscopy to confirm the essential structural characteristics (Fig. 4B). The CD spectra of all three chimeric proteins showed peak ellipticity values at $220 \mathrm{~nm}$ at $20^{\circ} \mathrm{C}$, while these peaks were not observed at $50^{\circ} \mathrm{C}$. Rotary shadowing images show that each chimeric rScl adopts a lollipop-like structural organization, with both CL-domain stalks and globular V domains. Thus, like whole loop substitutions, partial loop substitutions did not impact the structures of the chimeric rScls.

Binding data to $\mathrm{CFn}$ and rEDA indicated that only one of the three chimeras with partialloop-substitutions, rScl.chiCFL (C-terminal substitution), binds to cFn and rEDA, similar to the whole-loop-substitution construct rScl.chi1FL and the loop donor protein rScl1.1FL (Fig. 4C); the other two chimeric proteins, as well as binding-negative control protein rScl2.28FL, do not bind to cFn and rEDA. We observed substantially lower O.D. values recorded during rEDA binding to rScl.chi1FL and rScl.chiCFL, compared to rScl1.1FL. This may reflect less than optimal loop conformation in chimeric proteins associated with rScl2 backbone. Additional binding assays were carried out with increasing concentrations $(0.039-5.0 \mu \mathrm{M})$ of $r E D A$ ligand and $r S c l$ proteins to assess binding specificity (Fig. 4D). Concentration-dependent binding of rEDA to rScl.chi1FL, rScl.chiCFL, and control protein rScl1.1FL plateaued between 2.5 and $5.0 \mu \mathrm{M}$ concentration. In contrast, neither rScl.chiNFL, rScl.chilFL, rScl.chi2FL, nor control rScl2.28FL showed significant binding to rEDA. Therefore, these data provide evidence that the C-terminal 11 amino acids of $r S c l 1.1-\mathrm{V}$ loop are sufficient for conferring EDA/cFn binding, when inserted into binding-negative rScl2.28 protein.

\section{Distribution of the charged residues on the surface of $V$ domain hints at a binding} mechanism for EDA/cFn - Our initial examination of the multiple sequence alignment of Scl1- and Scl2-V domains failed to identify any reasonably conserved pattern of residues, which could be responsible for EDA/cFn binding (Fig. 1A). Our subsequent experiments, employing the chimeric loop sequences, highlighted a pattern of charged amino acids (e.g., $\mathrm{K}^{45(\mathrm{Scl1} 11)}, \mathrm{E}^{46,47(\mathrm{Scl1} 11)}$, and $\mathrm{D}^{\left.48(\mathrm{Scl1} 1)^{1}\right)}$ that were present within the uppermost portion of the Scl1-V loops and transitioning to inner a-helices, but were absent in the respective 
regions of the ECM-binding-negative Scl2-V loops (Fig. 1B). Therefore, we sought to investigate the surface potentials of the Scl1-, Scl2-, and the chimeric rScl.chi-V domains.

Electrostatic surface maps were computed from the homology models of the ECMbinding-positive and binding-negative proteins. Electrostatic maps computed for Scl1.1and Scl2.28-V domains reveal striking differences in the distribution of charges across their surfaces. A uniform, negatively-charged electrostatic potential characterizes the ECM-binding-negative Scl2.28-V on the frontal apical region of the molecule, opposite to the triple helix attachment (Fig. 5, black ovals). These charges are due to acidic residues located on the C-terminal part of the first outer $\alpha$-helix, the loop region, and $\mathrm{N}$-terminal part of the second inner $\alpha$-helix. In this same area, the electrostatic potential map of the binding-positive Scl1.1-V domain presents a central negatively charged pocket or cleft (Fig. 5, white arrowhead), surrounded by a neutral and basic crown surface. Although less pronounced, the same features of electrostatic potential, with a central negativelycharged region surrounded by a neutral/basic surface are present in maps of the bindingpositive chimeric rScl.chi1 and $\mathrm{rScl} . c h i C-\mathrm{V}$ domains, but not binding-negative chimeric $\mathrm{rScl}$ proteins. Therefore, the specific charge pattern of the $\mathrm{V}$-domain head strongly differentiates between binding-positive and binding-negative $V$ domains. These considerations suggest that interactions with partnering molecules occur through electrostatic recognition of the observed charge pattern.

\section{Scl1-V-loop is responsible for the ECM binding in phylogenetically distant Scl1} variants - Phylogenetic analysis of the amino acid sequences of the Scl1-V and Scl2-V domains show that both Scl1 and Scl2 form two distinct clusters (Fig. 6A). Within Scl1, there are two observed ligand-binding patterns. The first pattern is represented by Scl1 variants (Fig. 6A, black boxes) that bind host ECM proteins, cFn/EDA and Lm, (25) and ApoB100/LDL (20). The second pattern is represented by two Scl1 variants (Fig. 6A, black dots) that bind host complement components, CFH and CFHR1 $(26,27)$, suggesting the potential for a unique adaptation of Scl1 proteins in M6 and M55 GAS. In contrast, the majority of rScl1 constructs investigated, show the former binding pattern and these Scl1 variants are distributed all over many branches of the Scl1 cluster, highlighting the importance of ECM and LDL binding among GAS of various M types $(20,25,45,48,49)$. 
We next investigated whether the loop region of the phylogenetically distant Scl1.28-V variant, found in M28-type GAS, which shares $46 \%$ sequence identity with Scl1.1-V sequence (Fig. 6A, arrows), will support EDA/cFn binding. To do this, we substituted the loop region of Scl1.28-V (residues 25-46) in place of the loop region of Scl2.28-V (residues 20-45), resulting in the chimeric construct rScl.chi3FL (Fig. 6B). The CD spectra of rScl.chi3FL showed peak ellipticity at $220 \mathrm{~nm}$ at $20^{\circ} \mathrm{C}$, but not at $50^{\circ} \mathrm{C}$, and rotary shadowed images shows characteristic lollipop-like domain organization (Fig. 6C). Binding data to $\mathrm{cFn}$ and rEDA show that rScl.chi3FL binds both ligands (Fig. 6D). Thus, as in Scl1.1-V, the loop region of the divergent Scl1.28-V variant confers binding to both rEDA and cFn. Despite significant efforts, we could not generate a homology model of Scl1.28-V domain with a satisfactory global model quality estimation, due to the low sequence identity with the template structure of Scl2.28-V domain, close to $12 \%$.

The same structural constraints determine recognition and binding of the Scl1-V domain to laminin and apolipoprotein B100/LDL - Here, we sought to understand whether the same structural constraints apply during Scl1 binding to Lm and Apo B100/LDL (Fig. 7). Both rScl1.1FL and rScl1.3FL bound to Lm and ApoB100, while their respective V-domain-only counterparts, rScl1.1V and rScl1.3V, demonstrated negligible or no binding to both ligands (Fig. 7A). Chimeric constructs rScl.chi1FL, rScl.chiCFL, and rScl.chi3FL also bind to Lm and ApoB100/LDL, whereas binding-negative control proteins rScl2.28FL and rScl.chi2FL (reverse loop replacement to that in rScl.chi1FL) did not (Fig. $7 B, C$ ); in addition, both rScl.chiN and rScl.chil did not bind to Lm and ApoB100/LDL (data not shown). Collectively, our data show that: (i) full-length Scl1 protein is required for binding to all ligands; and (ii), the C-terminal 11 amino acids of the Scl1-V loop region is sufficient to mediate binding of Scl1 to its sequence- and structure-diverse ligands.

\section{DISCUSSION}

Adhesion to $\mathrm{Fn}$ is important for the pathogenesis of group A Streptococcus (7) by promoting the development of glycocalyx-embedded tissue microcolonies (in vivo biofilms) that are seen in clinical specimens collected from cases of pharyngitis and skin infections $(8,50)$. We previously reported that the streptococcal collagen-like protein 1 or 
Scl1 uniquely adheres to $\mathrm{cFn}$ isoforms expressed in wounded tissue, and that interactions involve the sequence-variable (V) globular domain of Scl1 and extra domain A (EDA) of cFn $(25,45)$. Here, we report on the structural determinants involved in Scl1 binding to $\mathrm{cFn}$, in the context of diversity among fibronectin-binding proteins of GAS and other bacterial pathogens. We also report that the same determinant of the Scl1 adhesin is responsible for binding to laminin (Lm) and apolipoprotein B100/low density lipoprotein (ApoB100/LDL) complex.

Several mammalian collagens, such as collagen type I, IV, and X, contain an Nterminal and/or C-terminal non-collagenous domain, which serves as a nucleation or trimerization domain, thereby facilitating proper folding of the three adjacent $\alpha$-chains into a mature collagen triple helix (51). Similarly, bacterial collagen-like proteins contain noncollagenous trimerization domains, often of unknown functions $(14,44)$. The BclA of Bacillus anthracis endospores possess a $\mathrm{C}$-terminal non-collagenous trimerization domain, BcIA-CTD, for CL-domain folding and increased thermal stability (52). Two different recombinant BclA-CTD constructs had the same crystal structure, similar to a non-collagenous globular domain of $\mathrm{C} 1 \mathrm{q}$, independent from whether these constructs included the continuous CL domain $(53,54)$. Likewise, the N-terminal V domains of Scl1 and Scl2 assisted in the trimerization of homologous and heterologous CL domains $(44,55)$, and the recombinant Scl2-V-only construct formed a trimeric 6 -helical bundle structure (22). However, here we found that the rScl1-V-only constructs, derived from the corresponding full-length rScl1FL proteins, did not bind rEDA, cFn, Lm, and ApoB100/LDL. We propose that the triple helix imposes a restraint on the $\mathrm{V}$ domain in a way to destabilize it and make it more prone to conformational changes. We expect a slight effect on the tertiary structure but not on the secondary structure. A reason of the restraint may be the one residue stagger of triple helices, whereas the $\mathrm{V}$ domains are perfectly aligned; these structural features likely distort the $V$ domain slightly, to make it more flexible.

While Scl1 is unique amongst GAS Fn-binding proteins in recognizing the EDA type III repeat of cFn, several other bacterial adhesins may in fact recognize the EDA, due to their selective binding to $\mathrm{cFn}$ but not $\mathrm{pFn}$, such as FimH of pathogenic E.coli, OppA and Msp of Treponema denticola, and Tp0155 of Treponema pallidum (56). In addition, 
numerous bacterial surface proteins bind Fn type III repeats other than EDA that are present in both pFn and cFn, including proteins ShdA, ComE1, Pap31, and Ali, that are associated with the outer membrane of gram-negative bacteria (57-60) and fibrillar surface proteins Embp and PavA-B of gram-positive bacteria $(61,62)$. Two pneumococcal Fn-binding proteins, PavA and PavB, bind to multiple Fnlll repeats via common epitopes located on $\beta$-strands $B, C$, and $F$, as well as the $A B$ and $B C$ loops (62). This is, however, different from the Scl1 binding site on EDA, mapped to C-C' loop (45). Interestingly, $\mathrm{PavA} / \mathrm{B}$ did not bind the RGD sequence on Fnlll ${ }_{10}$, which is recognized by the integrin $\alpha_{5} \beta_{1}$, nor to the synergistic sites on repeats III $_{8}$ and III (62), whereas the Scl1 binding site on C-C' loop maps in the vicinity of binding site for integrins $\alpha_{9} \beta_{1}$ and $\alpha_{4} \beta_{1}(38,45)$. PavA and PavB binding to the FnIII repeats was modeled to be largely due to electrostatic interactions; however, basic amino acids were primarily responsible for binding, rather than negatively charged residues, as modeled here for Scl1-V. Thus, Scl1 remains a unique adhesin that targets $\mathrm{EDA} / \mathrm{cFn}$ isoforms that are expressed at the pathogen portal of entry.

Here, we identified the loop region of the Scl1-V domain, as the structural determinant that is central to EDA/cFn recognition and binding. When the 22-amino-acid loop sequence of the EDA-binding positive Scl1-V variant, derived from the global M1type strain, was transplanted into the corresponding region of the binding-negative Scl2 protein, the chimeric construct gained EDA-binding activity. When the reverse transplantation was performed, ligand binding was lost both in recombinant protein and when expressed on the surface of Scl1 mutant GAS. A similar gain-of-binding function by the chimeric protein was achieved, when the loop-region from a phylogenetically distant Scl1 variant from M28-type GAS was substituted into the Scl2-V loop. A novel "catchclamp' Fn-binding mechanism was deciphered for the multifunctional filamentous adhesin of S. gordonii, CshA (63). The CshA protein, which also lacks the classical Fn-binding repeats, binds both $\mathrm{pFn}$ and $\mathrm{cFn}$ via its non-repetitive domains 1 and 2 . The NR2 globular domain has a $\beta$-sandwich structure with lectin-like fold, and likely binds glycosylated sites on Fn. The Fn-binding site of NR2 domain is capped by a flexible ligand-gating loop, which, upon ligand recognition, uncovers a presumed binding cleft. The Scl1-V domain is different, because it forms a 6 -helix bundle structure, not lectin-like fold, and binds non- 
glycosylated recombinant EDA protein; in addition, the EDA repeat in human cFn is not glycosylated (31). The lectin-like domain, containing ligand-gating loop capping the ligand-binding cleft, is represented in numerous fibrillar streptococcal adhesins, including S. mutans SpaA (64), S. gordonii SspB (65), S. agalactiae BspA (66), and M28-type $S$. pyogenes AspA (67), all correlated with the extended antigen I/II protein family. While the detailed Scl1 structure and structural organization is different from those of antigen I/II affiliates, there are several intangible similarities, where the elongated $C L$ domain projects the globular ligand-binding domain away from the cell surface, with ligand-engaging loop placed at the apex of the Scl1-V structure.

Homology modeling of the Scl1-V domain revealed that electrostatic surface potential and distribution of charged residues likely participate in ligand recognition and binding. A negatively charged region is formed in the center of the Scl-V trimer of ligandbinding-positive proteins modelled (Scl1.1, rScl.chi1, and rScl.chiC) that are surrounded by neutral and positively-charged residues. In contrast, the electrostatic models of the binding-negative Scl-V domains (Scl2.28, rScl.chi2, and rScl.chiN) display a uniform negative surface charge throughout the crown of the Scl-V domain. The arrangement of the surface potential observed in the Scl1-V domains was associated with a pattern of conserved negatively-charged amino acids, primarily $\mathrm{E}^{46,47(\mathrm{Sc} 11.1)}$ and $\mathrm{D}^{48(\mathrm{Sc} 11.1) \text {, located at }}$ the $\mathrm{C}$-terminus of the loop sequence at the junction with the a-helices forming the inner core of Scl1.1-V bundle. This charge distribution is reminiscent of the ligand-gating loops, capping negatively-charged binding clefts, proposed in CshA and BspA $(63,66)$. This conserved pattern is observed in Scl1 variants that are evolutionarily distant on the phylogenetic tree; for example, $E^{48,50}$ of Scl1.28 from M28 GAS. Therefore, phylogenetically distant Scl1 proteins, which have significant amino-acid sequence variation, exhibit conservation in the arrangement of charged amino acids, and similar ligand-binding partners.

We previously reported that Scl1-V domain bound EDA/cFn, Lm and ApoB100/LDL, thus, supporting a dichotomous nature of Scl1-ligand binding in tissue and blood (23). Here, we established that the same regions of the globular Scl1 domain are critical in binding with all three ligands. It is not unusual that bacterial adhesins are multifunctional and bind several distinct ligands. Such proteins typically contain clearly defined 
functional domains; for example, the ECM-binding protein of $S$. epidermidis Embp, discussed earlier, contains both the Fn-binding FIVAR domains and the G-related albumin binding GA domains (61). The collagen domain in streptococcal surface proteins is also found in association with additional domains; for instance, the pneumococcal protein PcIA (68), harbors both FIVAR and G5 (binds N-acetylglucosamine) domains, in addition to the collagen domain (23). The Scl1-V domain is different in that the short sequence-variable loop region and a conserved arrangement of the surface potential at the apical plane of Scl1-V structure, are involved in binding of assorted ligands that do not share substantial sequence nor structural similarities. These data, combined with previous molecular evolutionary analysis $(9,11)$, as well as structural and phylogenetic analyses $(20,22)$, indicate that retaining the conserved structure of the Scl1-V domain is the selective constraint, which influenced sequence variation.

Our work demonstrates several critical structure-function constraints that determine ligand binding capacity by the globular domain of a major group A streptococcal adhesin, Scl1. Current prospects for a global GAS vaccine, based on M protein, are problematic because more than $240 \mathrm{M}$-protein types have been reported, especially in regions outside North America and Europe, where vaccines are acutely needed. The EDA binding to diverse Scl1 variants could be developed into a nonconventional treatment option for GAS infections. The EDA-derived cyclic peptide bound Scl1 with an affinity in the micromolar range (48). The ongoing research explores the possibility of designing an improved EDA-derived peptide for targeting diverse GAS strains. This work provides an important structural understanding of Scl1 adhesin, which is essential during early stages of GAS infection, and lays the foundation for the development of non-vaccine inhibitors of Scl1-ligand interactions for the prevention of infection progression.

\section{EXPERIMENTAL PROCEDURES}

Production of recombinant streptococcal collagen-like proteins (rScls) - Gene cloning and rScl-protein production were performed in Escherichia coli TB1 and BL-21, respectively, grown in Luria-Bertani (LB) media (Fisher Scientific) with ampicillin (100 $\mu \mathrm{g}$ $\mathrm{mL}^{-1}$ ) at $37^{\circ} \mathrm{C}$. rScl-encoding clones, which were derived from the original scl alleles, were 
generated by PCR amplification from GAS genomic DNA; primers contained flanking sequences compatible E.coli expression vector pASK-IBA2 digested with Bsal. Clones encoding the chimeric $r S c l$ proteins were generated using synthetic double-stranded DNA fragments (gBlocks; Integrated DNA Technologies) flanked by PfMI and Bsal sites. All plasmids were verified by DNA sequencing. Plasmid constructs used in this study are described in Table 1 and gBlocks are shown in Table S1.

All $\mathrm{rScl}$ proteins were produced using the Strep-tag II cloning, expression, and purifications system (IBA-GmbH, Geottingen, Germany). Proteins were expressed with a C-terminal affinity tag and purified on Strep-Tactin sepharose, as described $(14,15)$. The full-length ( $\mathrm{rSclFL}$ ) and V-region-only ( $\mathrm{rSclV}$ ) constructs were made, as designated: rScl1.1FL and rScl1.1V are derived from Scl1 protein in M1-type strain MGAS6708 (14); rScl1.28FL and rScl2.28FL originate from M28 strains MGAS6274 (14); and rScl1.3FL and $\mathrm{rScl1} .3 \mathrm{~V}$ are derived from M3 strain MGAS315 (48). Both naturally-derived and chimeric $\mathrm{rScl}$ proteins were expressed in E. coli BL21 periplasm following the induction with anhydrotetracycline at $0.2 \mu \mathrm{g} \mathrm{mL}^{-1}$ for 3 hours. Cells were centrifuged and suspended in high sucrose buffer (100mM Tris-HCl, 1 mM EDTA, pH 8.0,500mM sucrose) or Cell Lytic B Buffer (Sigma) for separation of the periplasmic fraction and subsequent affinity purification. Purified proteins were analyzed by SDS-PAGE and stained with RAPIDstain ${ }^{\mathrm{TM}}$ (G-Biosciences); proteins were dialyzed against 25 mM HEPES, pH 8.0, and stored at $-20^{\circ} \mathrm{C}$. Protein concentrations were determined using Qubit fluorometric quantitation (ThermoFisher).

Recombinant extra domain A (rEDA) Production - Recombinant EDA (Table 1) was produced using the $\mathrm{pQE}-30 \mathrm{His}$-tag cloning, expression, and purification system (Qiagen) in the E.coli strain JM-109 (Promega), as described (69). E. coli containing rEDAencoding plasmid was grown in LB broth supplemented with ampicillin $\left(100 \mu \mathrm{g} / \mathrm{ml}^{-1}\right)$. Protein expression was induced with $1 \mathrm{mM}$ isopropyl $\beta$-D-1-thiogalactopyranoside for 3 hours. Cells were harvested by centrifugation, and pellets were frozen at $-20^{\circ} \mathrm{C}$ for 2 hours or overnight. Cells were next suspended in a lysis buffer ( $50 \mathrm{mM}$ Tris/HCL pH 8.0, $50 \mathrm{mM}$ $\mathrm{NaCl}, 2 \mathrm{mM} \mathrm{MgCl}$, 2\% Triton X-100, $10 \mathrm{mM} \beta$-mercaptoethanol, $0.2 \mathrm{mg} / \mathrm{ml}$ lysozyme, 1 EDTA-free protease inhibitor cocktail tablet [per $10 \mathrm{~mL}$ ] (Pierce), $1 \mathrm{mM}$ phenylmethane 
sulfonyl fluoride, $10 \mathrm{U} / \mathrm{ml} \mathrm{DNasel)} \mathrm{and} \mathrm{incubated} \mathrm{on} \mathrm{ice} \mathrm{for} 20$ minutes. Cell lysate was centrifuged at high speed (16000 $\times 20$ minutes) and supernatant was collected. Supernatant was mixed with Ni-NTA agarose resin (Qiagen) by inversion for 1.5 hours, and then poured into a column (Bio-Rad). Sample was washed with $50 x$ resin bed volume of the wash buffer ( $50 \mathrm{mM} \mathrm{NaH}_{2} \mathrm{PO}_{4}, 20 \mathrm{mM}$ imidazole, $500 \mathrm{mM} \mathrm{NaCl}$ ), and then rEDA protein was eluted in elution buffer ( $50 \mathrm{mM} \mathrm{NaH}_{2} \mathrm{PO}_{4}, 250 \mathrm{mM}$ imidazole, $300 \mathrm{mM} \mathrm{NaCl}$ ). Purified protein was dialyzed against 25 mM HEPES buffer, $\mathrm{pH} 8.0$ and protein concentration was measured with Qubit fluorometric quantitation; protein integrity and purity were assessed by $15 \%$ SDS-PAGE. Protein was stored at $-20^{\circ} \mathrm{C}$ until future use.

Rotary shadowing and electron microscopy - rScl proteins were rotary shadowed and analyzed by electron microscopy, as previously described (14). Briefly, recombinant proteins were dialyzed against $0.1 \mathrm{M}$ ammonium bicarbonate. $\mathrm{rScl}$ samples were mixed with glycerol to a final glycerol concentration of $70 \%(\mathrm{vol} / \mathrm{vol})$ and $100 \mu \mathrm{l}$ of each sample was nebulized with an airbrush onto freshly cleaved mica. Samples were then dried in a vacuum and rotary shadowed with carbon/platinum using an electron beam gun titled at an angle of 6 degrees, relative to the mica surface, in a Balzers BAE 250 evaporator. The replicas were backed with carbon at 90 degrees, and then were floated in distilled water and picked up onto bare 600-mesh copper grids. Photomicrographs were taken using a FEI G2 electron microscope operated at $120 \mathrm{kV}$ with a $30-\mu \mathrm{m}$ objective aperture.

Circular dichroism spectroscopy - Circular dichroism spectroscopy of $\mathrm{rScl}$ proteins was performed as previously described (14). Briefly, protein samples were dialyzed against 1x Dulbecco's phosphate buffered saline, $\mathrm{pH}$ 7.4. CD spectra were taken with a Jasco 810 spectropolarimeter, in a thermostatically controlled cuvette, with a path length of $0.5 \mathrm{~cm}$. Data were acquired at $10 \mathrm{~nm}$ per minute. Wavelength scans were performed from $240 \mathrm{~nm}$ to $190 \mathrm{~nm}$, at either $20^{\circ} \mathrm{C}$, or $50^{\circ} \mathrm{C}$ for denaturation.

Protein binding assays - rScl proteins $(0.5 \mu \mathrm{M}$ solutions) were immobilized onto StrepTactin-coated microplate wells for $1.5 \mathrm{~h}$ at room temperature and blocked with Trisbuffered saline supplemented with $1 \%$ fetal bovine serum albumin (BSA) overnight at $4^{\circ} \mathrm{C}$, 
followed by incubation with ECM ligands: cellular fibronectin (cFn) (Sigma), recombinant extra domain A (rEDA), murine laminin-111 (Lm) (Sigma), and Apolipoprotein B100 $(A p o B)$ and low-density lipoprotein (LDL) (Sigma). The no $\mathrm{rScl}$ controls were performed in BSA-coated wells for each ligand and each antibody used. Final ODs were normalized by subtracting the BSA controls in each experimental set-up.

In regular binding assays, ECM ligands were added to the rScl-immobilized wells at $1 \mu \mathrm{g}$ per well and incubated at room temperature for $1 \mathrm{~h}$. To assess concentrationdependent binding, rEDA was added to the wells at concentrations ranging between 0.039-5 $\mu \mathrm{M}$. Bound ECM ligands were detected with anti-cFn polyclonal antibody (Sigma/F3648; 1:4000), anti-His-Tag monoclonal antibody (Proteintech/66005; 1:100), anti-Lm polyclonal antibody (Sigma/L939; 1:500), and anti-ApoB (Chemicon/AB742; 1:500); followed by goat anti-rabbit (Bio-Rad/1721019; 1:2000), anti-mouse (BioRad/1721011; 1:1000), or rabbit anti-goat (Bio-Rad/1721034; 1:1000) secondary antibodies conjugated to horseradish peroxidase (HRP) (Bio-Rad). The HRP substrate, 2,20-azino-bis(3-ethylbenzthiazoline-6-sulphonic acid) (Fisher) was used and colorimetric reactions were recorded at $\mathrm{OD}_{415} \mathrm{~nm}$.

Protein modeling - Multiple sequence alignments of protein sequences were generated using MUSCLE and refined manually based on the results of secondary structure predictions. Predicted $\alpha$-helices represent the consensus between PSIPRED, PHD, and SSPRO. The phylogenetic tree of Scl1-V and Scl2-V regions was inferred based on multiple sequence alignments, using Neighbour-Joining method (adapted from (20)). Branch lengths were scaled to the distance computed using the Gonnet250 matrix, and bootstrap values (statistical support for given bifurcation) were calculated for all nodes.

The homology models of Scl1.1-V and Scl2.28-V domains were obtained after consensus-based sequence alignment using HMMer, against the amino acid sequence of Scl2.3-V domain with solved, crystal structure(22)(PDB accession code: $4 \mathrm{nsm}$ ). The homology models were built with MODELLER (70) and stereo-chemical quality of the models was improved by energy minimization with GROMACS (71). 
Complementation of M1 group A Streptococcus- The E.coli shuttle plasmid pSB027 (72) (Table 1) was used for in-trans complementation. The DNA fragment encompassing the scl1.1 coding sequence with upstream promoter was PCR-amplified using genomic DNA from the M1-type strain MGAS5005 (WT) with primers 232Reg and 232Rev_Bg/lI (Table S1). The amplified DNA was cloned into pSB027 between Bg/ll and Hindll sites, resulting in pSL620 (Table 1). The scl.chi2 sequence was cloned as a synthetic double stranded DNA fragment (gBlocks: Integrated DNA Technologies: SL621 gBlock, Table S1) between Spel and PpuM sites of pSL620, resulting in pSL621 (Table 1). Both pSL620 and pSL621 were electroporated into MGAS5005 $\square s c / 1$ mutant GAS (9) and transformants were selected on $\mathrm{BHI}$ agar containing $10 \mu \mathrm{g} / \mathrm{mL}^{-1}$ chloramphenicol; plasmids were verified by DNA sequencing.

GAS cultures were grown in Todd Hewitt broth (Becton Dickinson and Co) supplemented with $0.2 \%$ yeast extract (THY medium) and on Brain Heart Infusion agar (Becton Dickinson and $\mathrm{Co}$ ) at $37^{\circ} \mathrm{C}$ in an atmosphere with $5 \% \mathrm{CO}_{2}$. For antibiotic selection, chloramphenicol $\left(5 \mu \mathrm{g} \mathrm{mL}^{-1}\right)$ was added to the medium.

Western blot analysis - Expression of Scl1.1 and Scl.chi2 proteins was determined by western immunoblotting of the bacterial cell-wall protein fraction, as described previously $(9,10)$. Briefly, bacterial cultures were grown to an $\mathrm{OD}_{600}$ of 0.5 and cells were harvested by centrifugation. GAS cells were next digested with $100 \mu \mathrm{g}$ lysozyme and $5 \mathrm{U}$ mutanolysin and phenylmethylsulfonyl fluoride in a $20 \%$ sucrose buffer. $5-25 \mu \mathrm{L}$ of the sample supernatants were separated by SDS-PAGE and transferred to a nitrocellulose membrane. Detection of Scl1.1 and Scl.chi2 proteins was performed using rabbit antibody (1:2000) specific to the Scl1.1-V domain (9). Horseradish peroxidase-conjugated goat anti-rabbit IgG secondary antibody (Bio-Rad/1721019; 1:2000) was added to blots, followed by addition of Clarity ECL substrate (Bio-Rad) for detection. Images were acquired using a ChemiDoc Touch Imaging System (Bio-Rad).

Flow cytometry analysis - Determination of Scl-surface expression and rEDA binding by GAS cells was measured by flow cytometry. Bacteria grown to an OD600 of 0.5 were harvested by centrifugation and washed with flow cytometry buffer (phosphate-buffered 
saline containing $10 \%$ Todd-Hewitt broth with $0.2 \%$ yeast extract). For Scl-surface detection, the anti-Scl1.1-V antibody (9) was pre-absorbed with MGAS5005 $\Delta s c / 1$ cells and then $A b$ (1:10 dil.) was incubated with GAS cells tested for 30 minutes on ice. Cells were next washed and incubated with Allophycocyanin (APC)-conjugated donkey antirabbit antibody (Jackson ImmunoResearch; 1:150).

For rEDA binding, GAS cells tested were incubated with rEDA for 30 minutes at room temperature and bound rEDA was detected with anti-His-Tag monoclonal antibody (1:10 dil.) (Proteintech) pre-absorbed with MGAS5005 WT cells. Samples were then incubated with goat anti-mouse polyclonal antibody conjugated with Alexa Fluor ${ }^{\circledR} 568$ (ThermoFisher; $1: 150$ ). Cells were washed and fixed in $0.4 \%$ paraformaldehyde and stored at $4^{\circ} \mathrm{C}$ until analysis. 50,000 events were collected per sample using a BD LSRFortessa and data were analyzed with FCS Express Flow 6 software.

Statistical analyses - Statistics was performed using the two-tailed paired Student's $t$ test or Two-way ANOVA analyses. Significance was denoted at levels of ${ }^{*} P \leq 0.05,{ }^{* *} P$ $\leq 0.01$, or ${ }^{* * *} P \leq 0.001$. Error bars represent standard deviations with analyses based on three independent experimental repeats $(\mathrm{N}=3)$, each performed in triplicate technical replicates.

\section{ACKNOWLEDGMENTS}

We thank Paolo Fagone for assistance with rEDA purification, Kathleen Brundage for flow cytometry assistance, and Janusz Bujnicki for initial bioinformatic analyses. We thank Nyles Charon for the critical reading of the manuscript. Special thanks to the Shriners Hospital for Children for their support of the Micro-Imagining Center in the Portland Research Unit. Flow cytometry experiments were performed in the West Virginia University Flow Cytometry \& Single Cell Core Facility, which is supported by the National Institutes of Health equipment grant number S10OD016165 and the Institute of General Medicine Sciences of the National Institute of General Medicine of the National Institutes of Health under grant number P30GM103488 (CoBRE). This work was supported in part by the National Institutes of Health Grants Al50666 and Al083683, as well as West Virginia University HSC Bridge Grant Funding (SL); DHM was supported by the 
Integrative Graduate Education and Research Training (IGERT) Traineeship for Research and Education in Nanotoxicology under award number 1144676 and by the Dr. Jennifer Gossling Scholarship in Microbiology.

\section{REFERENCES}

1. Carapetis, J. R., Steer, A. C., Mulholland, E. K., and Weber, M. (2005) The global burden of group A streptococcal diseases. Lancet Infect. Dis. 5, 685-694

2. Pfoh, E., Wessels, M. R., Goldmann, D., and Lee, G. M. (2008) Burden and economic cost of group A streptococcal pharyngitis. Pediatrics 121, 229-234

3. Efstratiou, A., and Lamagni, T. (2016) Epidemiology of Streptococcus pyogenes. In Streptococcus pyogenes: Basic biology to clinical manifestations [Internet] (Ferretti, J. J., Stevens, D. L., and Fischetti, V. A. eds.), University of Oklahoma Health Sciences Center, Oklahoma City, OK.

4. Bisno, A. L., Rubin, F. A., Cleary, P. P., and Dale, J. B. (2005) Prospects for a Group A Streptococcal Vaccine: Rationale, Feasibility, and Obstacles--Report of a National Institute of Allergy and Infectious Diseases Workshop. Clin. Infect. Dis. 41, 1150-1156

5. Cunningham, M. W. (2000) Pathogenesis of group A streptococcal infections. Clin. Microbiol. Rev. 13, 470-511

6. Pavone, P., Parano, E., Rizzo, R., and Trifiletti, R. R. (2006) Autoimmune neuropsychiatric disorders associated with streptococcal infection: Sydenham chorea, PANDAS, and PANDAS variants. J. Child Neurol. 21, 727-736

7. Yamaguchi, M., Terao, Y., and Kawabata, S. (2013) Pleiotropic virulence factor - Streptococcus pyogenes fibronectin-binding proteins. Cell. Microbiol. 15, 503-511

8. Akiyama, H., Morizane, S., Yamasaki, O., Oono, T., and Iwatsuki, K. (2003) Assessment of Streptococcus pyogenes microcolony formation in infected skin by confocal laser scanning microscopy. J. Dermatol. Sci. 32, 193-199

9. Lukomski, S., Nakashima, K., Abdi, I., Cipriano, V. J., Ireland, R. M., Reid, S. D., Adams, G. G., and Musser, J. M. (2000) Identification and characterization of the $s c /$ gene encoding a group $A$ Streptococcus extracellular protein virulence factor with similarity to human collagen. Infect. Immun. 68, 6542-6553

10. Lukomski, S., Nakashima, K., Abdi, I., Cipriano, V. J., Shelvin, B. J., Graviss, E. A., and Musser, J. M. (2001) Identification and characterization of a second extracellular collagen-like protein made by group A Streptococcus: control of production at the level of translation. Infect. Immun. 69, 1729-1738 
11. Rasmussen, M., Edén, A., and Björck, L. (2000) SclA, a novel collagen-like surface protein of Streptococcus pyogenes. Infect. Immun. 68, 6370-6377

12. Whatmore, A. M. (2001) Streptococcus pyogenes sclB encodes a putative hypervariable surface protein with a collagen-like repetitive structure. Microbiology 147, 419-429

13. Rasmussen, M., and Björck, L. (2001) Unique regulation of SclB - a novel collagen-like surface protein of Streptococcus pyogenes. Mol. Microbiol. 40, 1427-1438

14. Xu, Y., Keene, D. R., Bujnicki, J. M., Höök, M., and Lukomski, S. (2002) Streptococcal Scl1 and Scl2 proteins form collagen-like triple helices. J Biol Chem 277, 27312-27318

15. Han, R., Zwiefka, A., Caswell, C. C., Xu, Y., Keene, D. R., Lukomska, E., Zhao, Z., Höök, M., and Lukomski, S. (2006) Assessment of prokaryotic collagen-like sequences derived from streptococcal Scl1 and Scl2 proteins as a source of recombinant GXY polymers. Appl. Microbiol. Biotechnol. 72, 109-115

16. Mohs, A., Silva, T., Yoshida, T., Amin, R., Lukomski, S., Inouye, M., and Brodsky, B. (2007) Mechanism of stabilization of a bacterial collagen triple helix in the absence of hydroxyproline. $J$ Biol Chem 282, 29757-29765

17. Humtsoe, J. O., Kim, J. K., Xu, Y., Keene, D. R., Höök, M., Lukomski, S., and Wary, K. K. (2005) A streptococcal collagen-like protein interacts with the $\alpha 2 \beta 1$ integrin and induces intracellular signaling. J. Biol. Chem. 280, 13848-13857

18. Caswell, C. C., Lukomska, E., Seo, N. S., Höök, M., and Lukomski, S. (2007) Scl1-dependent internalization of group A Streptococcus via direct interactions with the $\alpha 2 \beta 1$ integrin enhances pathogen survival and re-emergence. Mol. Microbiol. 64, 1319-1331

19. Caswell, C. C., Barczyk, M., Keene, D. R., Lukomska, E., Gullberg, D. E., and Lukomski, S. (2008) Identification of the first prokaryotic collagen sequence motif that mediates binding to human collagen receptors, integrins $\alpha 2 \beta 1$ and $\alpha 11 \beta 1$. J Biol Chem 283, 36168-36175

20. Han, R., Caswell, C. C., Lukomska, E., Keene, D. R., Pawlowski, M., Bujnicki, J. M., Kim, J. K., and Lukomski, S. (2006) Binding of the low-density lipoprotein by streptococcal collagen-like protein Scl1 of Streptococcus pyogenes. Mol. Microbiol. 61, 351-367

21. Squeglia, F., Bachert, B., Romano, M., Lukomski, S., and Berisio, R. (2013) Crystallization and preliminary X-ray crystallographic analysis of the variable domain of Scl2.3, a streptococcal collagen-like protein from invasive M3-type Streptococcus pyogenes. Acta Crystallogr. Sect. F Struct. Biol. Cryst. Commun. 69, 1023-1025

22. Squeglia, F., Bachert, B., De Simone, A., Lukomski, S., and Berisio, R. (2014) The crystal structure of the streptococcal collagen-like protein 2 globular domain from invasive M3-type group A Streptococcus shows significant similarity to immunomodulatory HIV protein gp41. J Biol Chem 289, 5122-5133

23. Lukomski, S., Bachert, B. A., Squeglia, F., and Berisio, R. (2017) Collagen-like proteins of pathogenic streptococci. Mol. Microbiol. 103, 919-930 
24. Påhlman, L. I., Marx, P. F., Mörgelin, M., Lukomski, S., Meijers, J. C., and Herwald, H. (2007) Thrombin-activatable fibrinolysis inhibitor binds to Streptococcus pyogenes by interacting with collagen-like proteins A and B. J Biol Chem 282, 24873-24881

25. Caswell, C. C., Oliver-Kozup, H., Han, R., Lukomska, E., and Lukomski, S. (2010) Scl1, the multifunctional adhesin of group A Streptococcus, selectively binds cellular fibronectin and laminin, and mediates pathogen internalization by human cells. FEMS Microbiol. Lett. 303, 61-68

26. Caswell, C. C., Han, R., Hovis, K. M., Ciborowski, P., Keene, D. R., Marconi, R. T., and Lukomski, S. (2008) The Scl1 protein of M6-type group A Streptococcus binds the human complement regulatory protein, factor $\mathrm{H}$, and inhibits the alternative pathway of complement. Mol. Microbiol. 67, 584-596

27. Reuter, M., Caswell, C. C., Lukomski, S., and Zipfel, P. F. (2010) Binding of the human complement regulators CFHR1 and factor $\mathrm{H}$ by streptococcal collagen-like protein 1 (Scl1) via their conserved $\mathrm{C}$ termini allows control of the complement cascade at multiple levels. $\mathrm{J}$ Biol Chem 285, 38473-38485

28. Gao, Y., Liang, C., Zhao, R., Lukomski, S., and Han, R. (2010) The Scl1 of M41-type group A Streptococcus binds the high-density lipoprotein. FEMS Microbiol. Lett. 309, 55-61

29. Pankov, R., and Yamada, K. M. (2002) Fibronectin at a glance. J. Cell Sci. 115, 3861-3863

30. Ffrench-Constant, C. (1995) Alternative splicing of fibronectin--many different proteins but few different functions. Exp. Cell Res. 221, 261-271

31. Hynes, R. O. (1990) Fibronectins, Springer-Verlag, New York, NY

32. Ffrench-Constant, C., and Hynes, R. O. (1989) Alternative splicing of fibronectin is temporally and spatially regulated in the chicken embryo. Development 106, 375-388

33. Coito, A. J., Brown, L. F., Peters, J. H., Kupiec-Weglinski, J. W., and Van De Water, L. (1997) Expression of fibronectin splicing variants in organ transplantation: a differential pattern between rat cardiac allografts and isografts. Am. J. Pathol. 150, 1757-1772

34. Ffrench-Constant, C., Van De Water, L., Dvorak, H. F., and Hynes, R. O. (1989) Reappearance of an embryonic pattern of fibronectin splicing during wound healing in the adult rat. J. Cell Biol. 109, 903-914

35. Leahy, D. J., Aukhil, I., and Erickson, H. P. (1996) $2.0 \AA$ A crystal structure of a four-domain segment of human fibronectin encompassing the RGD loop and synergy region. Cell 84, 155-164

36. Niimi, T., Osawa, M., Yamaji, N., Yasunaga, K., Sakashita, H., Mase, T., Tanaka, A., and Fujita, S. (2001) NMR structure of human fibronectin EDA. J. Biomol. NMR 21, 281-284

37. Liao, Y. F., Gotwals, P. J., Koteliansky, V. E., Sheppard, D., and Van De Water, L. (2002) The EIIIA segment of fibronectin is a ligand for integrins $\alpha 9 \beta 1$ and $\alpha 4 \beta 1$ providing a novel mechanism for regulating cell adhesion by alternative splicing. J Biol Chem 277, 14467-14474 
38. Shinde, A. V., Bystroff, C., Wang, C., Vogelezang, M. G., Vincent, P. A., Hynes, R. O., and Van De Water, L. (2008) Identification of the peptide sequences within the EIIIA (EDA) segment of fibronectin that mediate integrin a9b1-dependent cellular activities. J Biol Chem 283, 2858-2870

39. Palmer, E. L., Ruegg, C., Ferrando, R., Pytela, R., and Sheppard, D. (1993) Sequence and tissue distribution of the integrin $\alpha 9$ subunit, a novel partner of $\beta 1$ that is widely distributed in epithelia and muscle. J. Cell Biol. 123, 1289-1297

40. Singh, P., Reimer, C. L., Peters, J. H., Stepp, M. A., Hynes, R. O., and Van De Water, L. (2004) The spatial and temporal expression patterns of integrin $\alpha 9 \beta 1$ and one of its ligands, the EIIIA segment of fibronectin, in cutaneous wound healing. J. Invest. Dermatol. 123, 1176-1181

41. Singh, P., Chen, C., Pal-Ghosh, S., Stepp, M. A., Sheppard, D., and Van De Water, L. (2009) Loss of integrin $\alpha 9 \beta 1$ results in defects in proliferation, causing poor re-epithelialization during cutaneous wound healing. J. Invest. Dermatol. 129, 217-228

42. Shinde, A. V., Kelsh, R., Peters, J. H., Sekiguchi, K., Van De Water, L., and McKeown-Longo, P. J. (2015) The $\alpha 4 \beta 1$ integrin and the EDA domain of fibronectin regulate a profibrotic pheotype in dermal fibroblasts Matrix Biol. 41, 26-35

43. Longmate, W. M., Lyons, S. P., Chittur, S. V., Pumiglia, K. M., Van De Water, L., and DiPersio, C. M. (2017) Suppression of integrin $\alpha 3 \beta 1$ by $\alpha 9 \beta 1$ in the epidermis controls the paracrine resolution of wound angiogenesis. J. Cell Biol. 216, 1473-1488

44. Yu, Z., Mirochnitchenko, O., Xu, C., Yoshizumi, A., Brodsky, B., and Inouye, M. (2010) Noncollagenous region of the streptococcal collagen-like protein is a trimerization domain that supports refolding of adjacent homologous and heterologous collagenous domains. Protein Sci. 19, 775-785

45. Oliver-Kozup, H., Martin, K. H., Schwegler-Berry, D., Green, B. J., Betts, C., Shinde, A. V., Van De Water, L., and Lukomski, S. (2013) The group A streptococcal collagen-like protein-1, Scl1, mediates biofilm formation by targeting the extra domain A-containing variant of cellular fibronectin expressed in wounded tissue. Mol. Microbiol. 87, 672-689

46. Brodsky-Doyle, B., Leonard, K. R., and Reid, K. B. (1976) Circular-dichroism and electronmicroscopy studies of human subcomponent $\mathrm{C} 1 \mathrm{q}$ before and after limited proteolysis by pepsin. Biochem. J. 159, 279-286

47. Engel, J., and Furthmayr, H. (1987) Electron microscopy and other physical methods for the characterization of extracellular matrix components: laminin, fibronectin, collagen IV, collagen $\mathrm{VI}$, and proteoglycans. Methods Enzymol. 145, 3-78

48. Bachert, B. A., Choi, S. J., LaSala, P. R., Harper, T. I., McNitt, D. H., Boehm, D. T., Caswell, C. C., Ciborowski, P., Keene, D. R., Flores, A. R., Musser, J. M., Squeglia, F., Marasco, D., Berisio, R., and Lukomski, S. (2016) Unique footprint in the scl1.3 locus affects adhesion and biofilm formation of the invasive M3-type group A Streptococcus. Front Cell Infect Microbiol 6, 90 
49. Chen, S. M., Tsai, Y. S., Wu, C. M., Liao, S. K., Wu, L. C., Chang, C. S., Liu, Y. H., and Tsai, P. J. (2010) Streptococcal collagen-like surface protein 1 promotes adhesion to the respiratory epithelial cell. BMC Microbiol. 10, 320

50. Roberts, A. L., Connolly, K. L., Kirse, D. J., Evans, A. K., Poehling, K. A., Peters, T. R., and Reid, S. D. (2012) Detection of group A Streptococcus in tonsils from pediatric patients reveals high rate of asymptomatic streptococcal carriage. BMC Pediatr. 12, 3

51. Bourhis, J.-M., Mariano, N., Zhao, Y., Harlos, K., Exposito, J.-Y., Jones, E. Y., Moali, C., Aghajari, N., and Hulmes, D. J. S. (2012) Structural basis of fibrillar collagen trimerization and related genetic disorders. Nature Structural \&Amp; Molecular Biology 19, 1031

52. Boydston, J. A., Chen, P., Steichen, C. T., and Turnbough, C. L., Jr. (2005) Orientation within the exosporium and structural stability of the collagen-like glycoprotein BclA of Bacillus anthracis. J. Bacteriol. 187, 5310-5317

53. Réty, S., Salamitou, S., Garcia-Verdugo, I., Hulmes, D. J., Le Hégarat, F., Chaby, R., and LewitBentley, A. (2005) The crystal structure of the Bacillus anthracis spore surface protein BclA shows remarkable similarity to mammalian proteins. J Biol Chem 280, 43073-43078

54. Liu, C. Q., Nuttall, S. D., Tran, H., Wilkins, M., Streltsov, V. A., and Alderton, M. R. (2008) Construction, crystal structure and application of a recombinant protein that lacks the collagenlike region of BcIA from Bacillus anthracis spores. Biotechnol. Bioeng. 99, 774-782

55. Xu, C., Yu, Z., Inouye, M., Brodsky, B., and Mirochnitchenko, O. (2010) Expanding the family of collagen proteins: recombinant bacterial collagens of varying composition form triple-helices of similar stability. Biomacromolecules 11, 348-356

56. Henderson, B., Nair, S., Pallas, J., and Williams, M. A. (2011) Fibronectin: a multidomain host adhesin targeted by bacterial fibronectin-binding proteins. FEMS Microbiol. Rev. 35, 147-200

57. Kingsley, R. A., Keestra, A. M., de Zoete, M. R., and Baumler, A. J. (2004) The ShdA adhesin binds to the cationic cradle of the fibronectin 13Fnlll repeat module: evidence for molecular mimicry of heparin binding. Mol. Microbiol. 52, 345-355

58. Mullen, L. M., Nair, S. P., Ward, J. M., Rycroft, A. N., Williams, R. J., Robertson, G., Mordan, N. J., and Henderson, B. (2008) Novel adhesin from Pasteurella multocida that binds to the integrinbinding fibronectin FnIII9-10 repeats. Infect. Immun. 76, 1093-1104

59. Dabo, S. M., Confer, A. W., Anderson, B. E., and Gupta, S. (2006) Bartonella henselae Pap31, an extracellular matrix adhesin, binds the fibronectin repeat III13 module. Infect. Immun. 74, 2513-2521

60. Tsang, T. M., Annis, D. S., Kronshage, M., Fenno, J. T., Usselman, L. D., Mosher, D. F., and Krukonis, E. S. (2012) Ail protein binds ninth type III fibronectin repeat (9FNIII) within central 120$\mathrm{kDa}$ region of fibronectin to facilitate cell binding by Yersinia pestis. J Biol Chem 287, 1675916767 
61. Christner, M., Franke, G. C., Schommer, N. N., Wendt, U., Wegert, K., Pehle, P., Kroll, G., Schulze, C., Buck, F., Mack, D., Aepfelbacher, M., and Rohde, H. (2010) The giant extracellular matrix-binding protein of Staphylococcus epidermidis mediates biofilm accumulation and attachment to fibronectin. Mol. Microbiol. 75, 187-207

62. Kanwal, S., Jensch, I., Palm, G. J., Brönstrup, M., Rohde, M., Kohler, T. P., Somplatzki, D., Tegge, W., Jenkinson, H. F., and Hammerschmidt, S. (2017) Mapping the recognition domains of pneumococcal fibronectin-binding proteins PavA and PavB demonstrates a common pattern of molecular interactions with fibronectin type III repeats. Mol. Microbiol. 105, 839-859

63. Back, C. R., Sztukowska, M. N., Till, M., Lamont, R. J., Jenkinson, H. F., Nobbs, A. H., and Race, P. R. (2017) The Streptococcus gordonii adhesin CshA protein binds host fibronectin via a catchclamp mechanism. J Biol Chem 292, 1538-1549

64. Troffer-Charlier, N., Ogier, J., Moras, D., and Cavarelli, J. (2002) Crystal structure of the V-region of Streptococcus mutans antigen I/II at 2.4 A resolution suggests a sugar preformed binding site. J. Mol. Biol. 318, 179-188

65. Forsgren, N., Lamont, R. J., and Persson, K. (2009) Crystal structure of the variable domain of the Streptococcus gordonii surface protein SspB. Protein Sci. 18, 1896-1905

66. Rego, S., Heal, T. J., Pidwill, G. R., Till, M., Robson, A., Lamont, R. J., Sessions, R. B., Jenkinson, H. F., Race, P. R., and Nobbs, A. H. (2016) Structural and functional analysis of cell wall-anchored polypeptide adhesin BspA in Streptococcus agalactiae. J. Biol. Chem. 291, 1598516000

67. Green, N., Zhang, S., Porcella, S., Nagiec, M., Barbian, K., Beres, S., Lefebvre, R., and Musser, J. (2005) Genome sequence of a serotype M28 strain of group A Streptococcus: potential new insights into puerperal sepsis and bacterial disease specificity. J. Infect. Dis. 192, 760-770

68. Paterson, G. K., Nieminen, L., Jefferies, J. M. C., and Mitchell, T. J. (2008) PclA, a pneumococcal collagen-like protein with selected strain distribution, contributes to adherence and invasion of host cells. FEMS Microbiol. Lett. 285, 170-176

69. Kelsh, R., You, R., Horzempa, C., Zheng, M., and McKeown-Longo, P. J. (2014) Regulation of the innate immune response by fibronectin: synergism between the III-1 and EDA domains. PLoS One 9, e102974

70. Webb, B., and Sali, A. (2017) Protein structure modeling with MODELLER. Methods Mol. Biol. 1654, 39-54

71. Pronk, S., Páll, S., Schulz, R., Larsson, P., Bjelkmar, P., Apostolov, R., Shirts, M. R., Smith, J. C., Kasson, P. M., van der Spoel, D., Hess, B., and Lindahl, E. (2013) GROMACS 4.5: a highthroughput and highly parallel open source molecular simulation toolkit. Bioinformatics 29, 845854 
72. Cramer, T., Yamanishi, Y., Clausen, B. E., Förster, I., Pawlinski, R., Mackman, N., Haase, V. H., Jaenisch, R., Corr, M., Nizet, V., Firestein, G. S., Gerber, H. P., Ferrara, N., and Johnson, R. S. (2003) HIF-1 $\alpha$ is essential for myeloid cell-mediated inflammation. Cell 112, 645-657

73. Flores, A. R., Jewell, B. E., Versalovic, E. M., Olsen, R. J., Bachert, B. A., Lukomski, S., and Musser, J. M. (2015) Natural variant of collagen-like protein A in serotype M3 Group A

Streptococcus increases adherence and decreases invasive potential. Infect. Immun. 83, 11221129 


\section{TABLES}

Table1: Plasmids used in this study.

\begin{tabular}{|c|c|c|}
\hline Plasmid & Description & Source \\
\hline pASK-IBA2 & $\begin{array}{l}\text { E. coli expression vector, } \\
\text { C-terminal Strep-tag II }\end{array}$ & IBA, Göttingen \\
\hline pSL503 & $\begin{array}{l}\text { pASK-IBA2 encoding } \\
\text { rScl1.3FL }\end{array}$ & (73) \\
\hline pSL514 & $\begin{array}{l}\text { pASK-IBA2 encoding } \\
\text { rScl1.3V }\end{array}$ & (48) \\
\hline pSL144 & $\begin{array}{l}\text { pASK-IBA2 encoding } \\
\text { rScl1.1FL }\end{array}$ & (14) \\
\hline pSL158 & $\begin{array}{l}\text { pASK-IBA2 encoding } \\
\text { rScl1.1V }\end{array}$ & (14) \\
\hline pSL161 & $\begin{array}{l}\text { pASK-IBA2 encoding } \\
\text { rScl1.28FL }\end{array}$ & (15) \\
\hline pSL163 & $\begin{array}{l}\text { pASK-IBA2 encoding } \\
\text { rScl2.28FL }\end{array}$ & (14) \\
\hline pSL600 & $\begin{array}{l}\text { pASK-IBA2 encoding } \\
\text { rScl.chi1FL }\end{array}$ & This study \\
\hline pSL601 & $\begin{array}{l}\text { pASK-IBA2 encoding } \\
\text { rScl.chiNFL }\end{array}$ & This study \\
\hline pSL602 & $\begin{array}{l}\text { pASK-IBA2 encoding } \\
\text { rScl.chiCFL }\end{array}$ & This study \\
\hline pSL603 & $\begin{array}{l}\text { pASK-IBA2 encoding } \\
\text { rScl.chilFL }\end{array}$ & This study \\
\hline pSL613 & $\begin{array}{l}\text { pASK-IBA2 encoding } \\
\text { rScl.chi2FL }\end{array}$ & This study \\
\hline pSL614 & $\begin{array}{l}\text { pASK-IBA2 encoding } \\
\text { rScl.chi3FL }\end{array}$ & This study \\
\hline pQE-30 & $\begin{array}{l}\text { N-terminal His-tag E. coli } \\
\text { expression vector }\end{array}$ & Qiagen \\
\hline pQE-EDA & pQE-30 encoding rEDA & (69) \\
\hline pSB027 & $\begin{array}{l}\text { E. coli -GAS shuttle } \\
\text { plasmid encoding GFP }\end{array}$ & (72) \\
\hline pSL620 & $\begin{array}{l}\text { pSB027 encoding scl1.1 } \\
\text { allele from MGAS } 5005\end{array}$ & This study \\
\hline pSL621 & $\begin{array}{l}\text { pSL620 encoding } \\
\text { Scl.chi2FL }\end{array}$ & This study \\
\hline
\end{tabular}




\section{Figure 1}

\section{FIGURES}

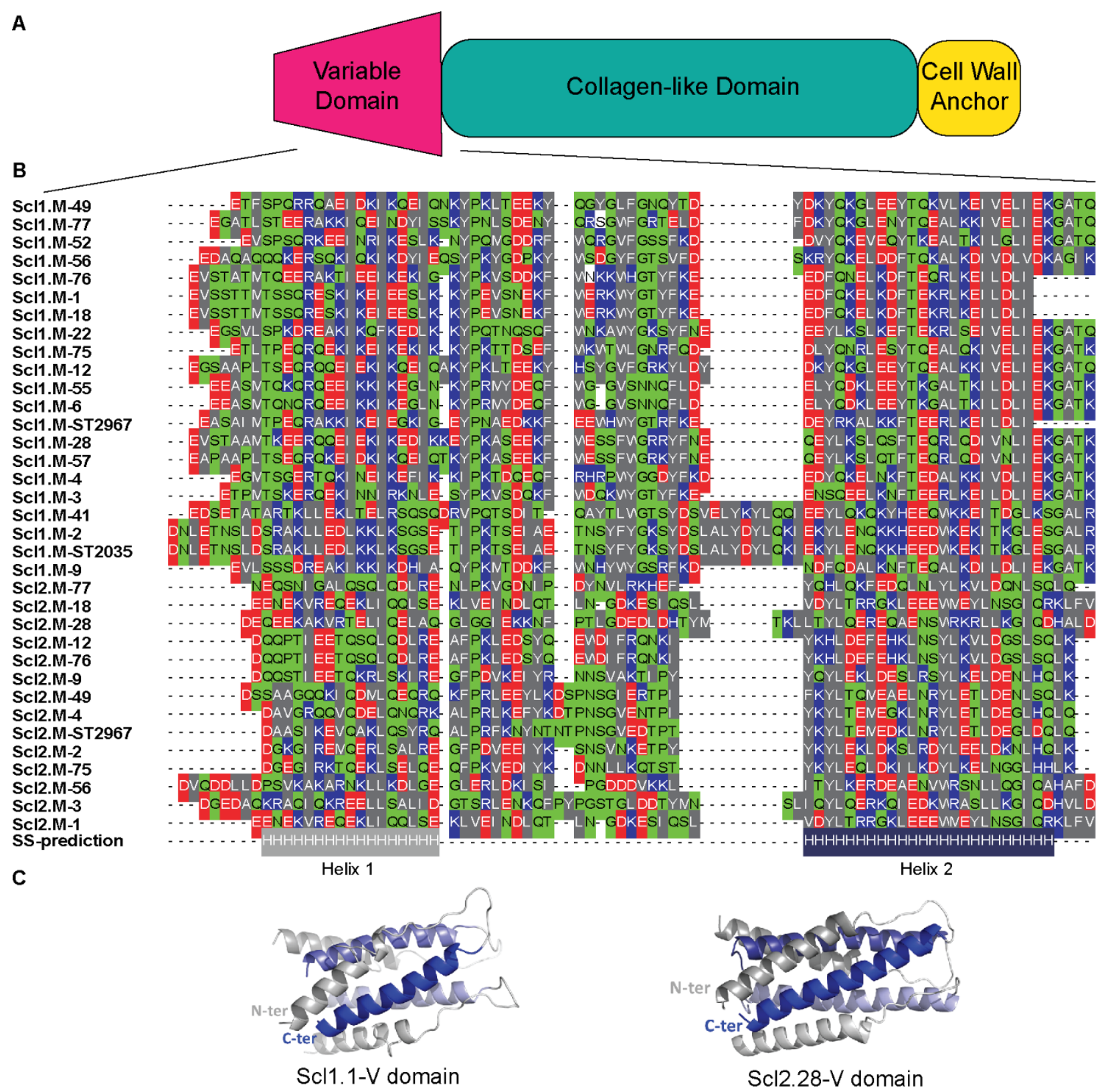

Figure 1. Conserved structure of the Scl1 and Scl2 globular domains. $(A)$ Cartoon model of full-length $\mathrm{Scl}$ proteins. Globular N-terminal sequence-variable (V) domain, central rod-shaped collagen $(\mathrm{CL})$ domain, and $\mathrm{C}$-terminal cell-wall-anchor domain are shown. $(B)$ Multiple sequence alignment of the $\mathrm{V}$ regions of Scl1 and Scl2 variants. MSA was generated using MUSCLE; amino acid residues are colored according to their physico-chemical character: red - negative $(D, E)$, blue - positive $(R, K, H)$, gray - hydrophobic, 
aliphatic or aromatic (I, L, V, A, M, F, Y, W), and green - polar, Gly, and Pro (S, T, Q, N, G, P). Predicted alpha helices: $\mathrm{N}$-terminal helix 1 (grey) and C-terminal helix 2 (blue) are shown below the alignments $(\mathrm{H})$. (C) Homology models of the Scl1.1-V and Scl2.28-V domains. Models were generated based on the crystal structure of the Scl2.3-V variant (PDB accession code: $4 \mathrm{nsm}$ ). Modeled alpha helices: $\mathrm{N}$-terminal helix 1 (grey) and C-terminal helix 2 (blue) are shown. 


\section{Figure 2}

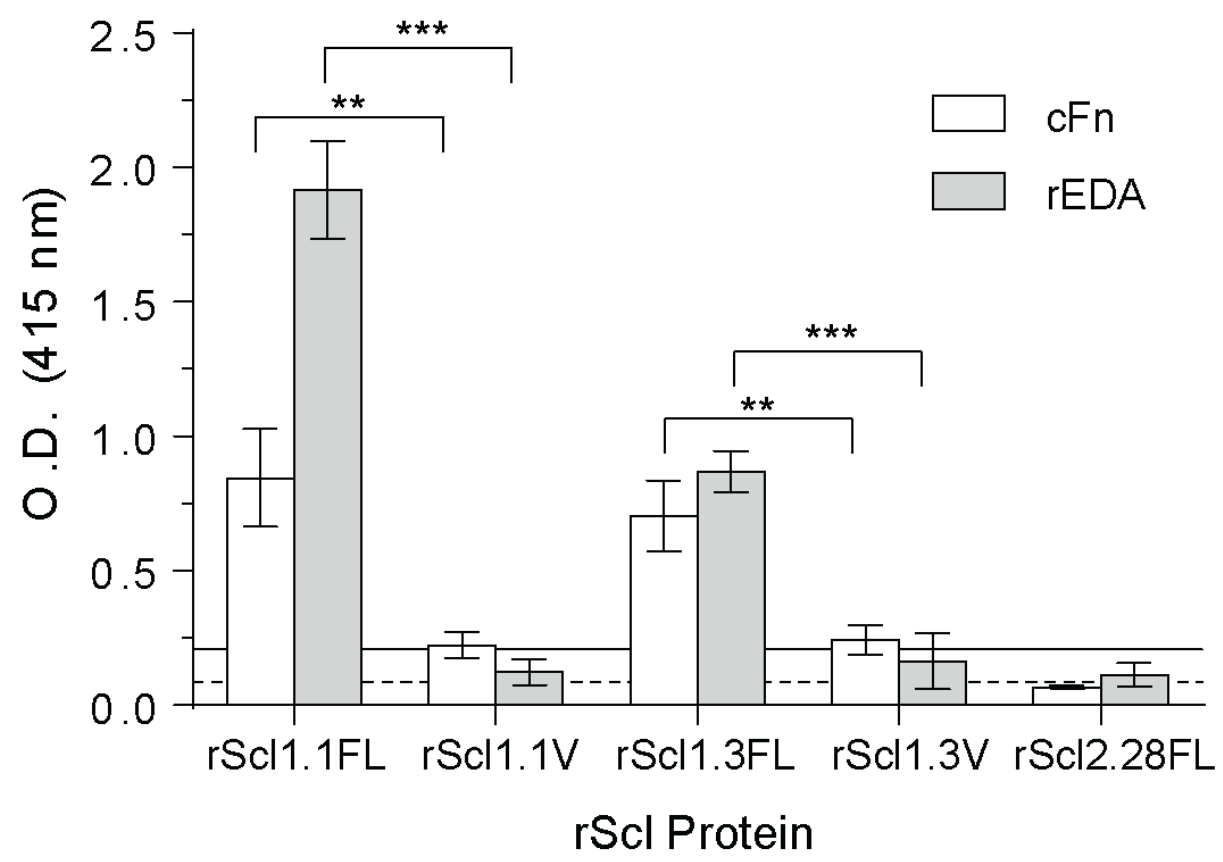

Figure 2. Full-length Scl1 proteins are required for binding to ECM ligands. Recombinant full-length proteins ( $\mathrm{rScl1} .1 \mathrm{FL}$ and $\mathrm{rScl1} .3 \mathrm{FL}$ ) and their respective V-region-only constructs ( $\mathrm{rScl1} .1 \mathrm{~V}$ and $\mathrm{rScl1} .3 \mathrm{~V}$ ) were tested for binding to cellular fibronectin (cFn) and recombinant extra domain A (rEDA). (A) ELISA binding assay. rScl proteins were immobilized onto Strep-Tactin-coated microplate wells and incubated with ECM ligands, cFn and rEDA. Primary anti-ECM Abs and HRP-conjugated secondary Abs were used for ligand detection. Graphic bars indicate the mean $\mathrm{OD}_{415 \mathrm{~nm}}$ normalized against control BSA-coated wells. Statistical analysis was calculated using Student's two-tailed $t$-test from three independent experiments, each performed in triplicate wells ( $N=3 \pm \mathrm{SD}$ ); ${ }^{* \star} P \leq 0.01$, ${ }^{* * *} P \leq 0.001$. Solid (rEDA) and dashed (cFn) lines indicate threshold $\mathrm{OD}_{415} \mathrm{~nm} \pm 2 \mathrm{SD}$ values recorded for experimentally validated ECM-binding-negative control protein rScl2.28FL. 


\section{Figure 3}

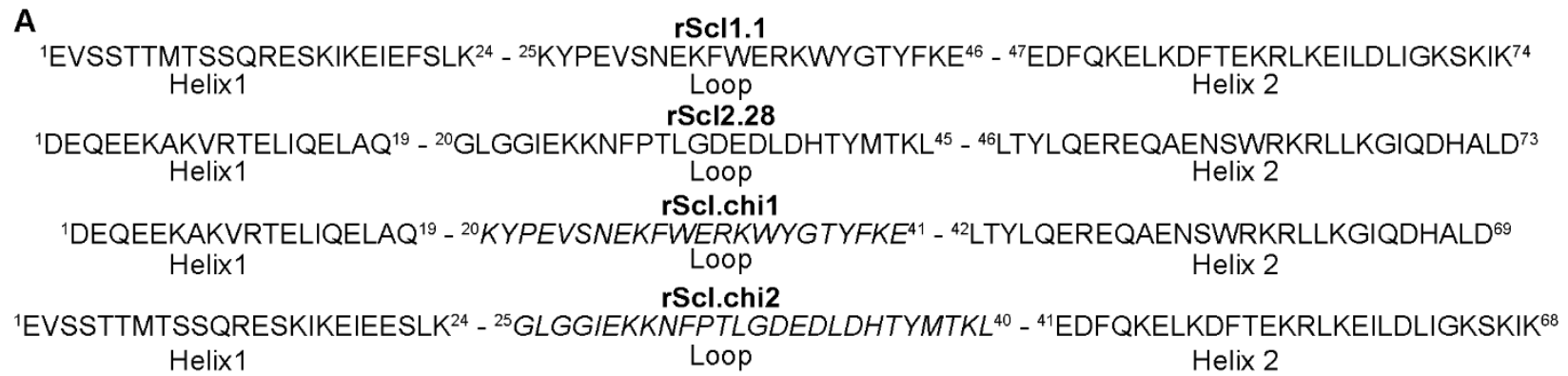
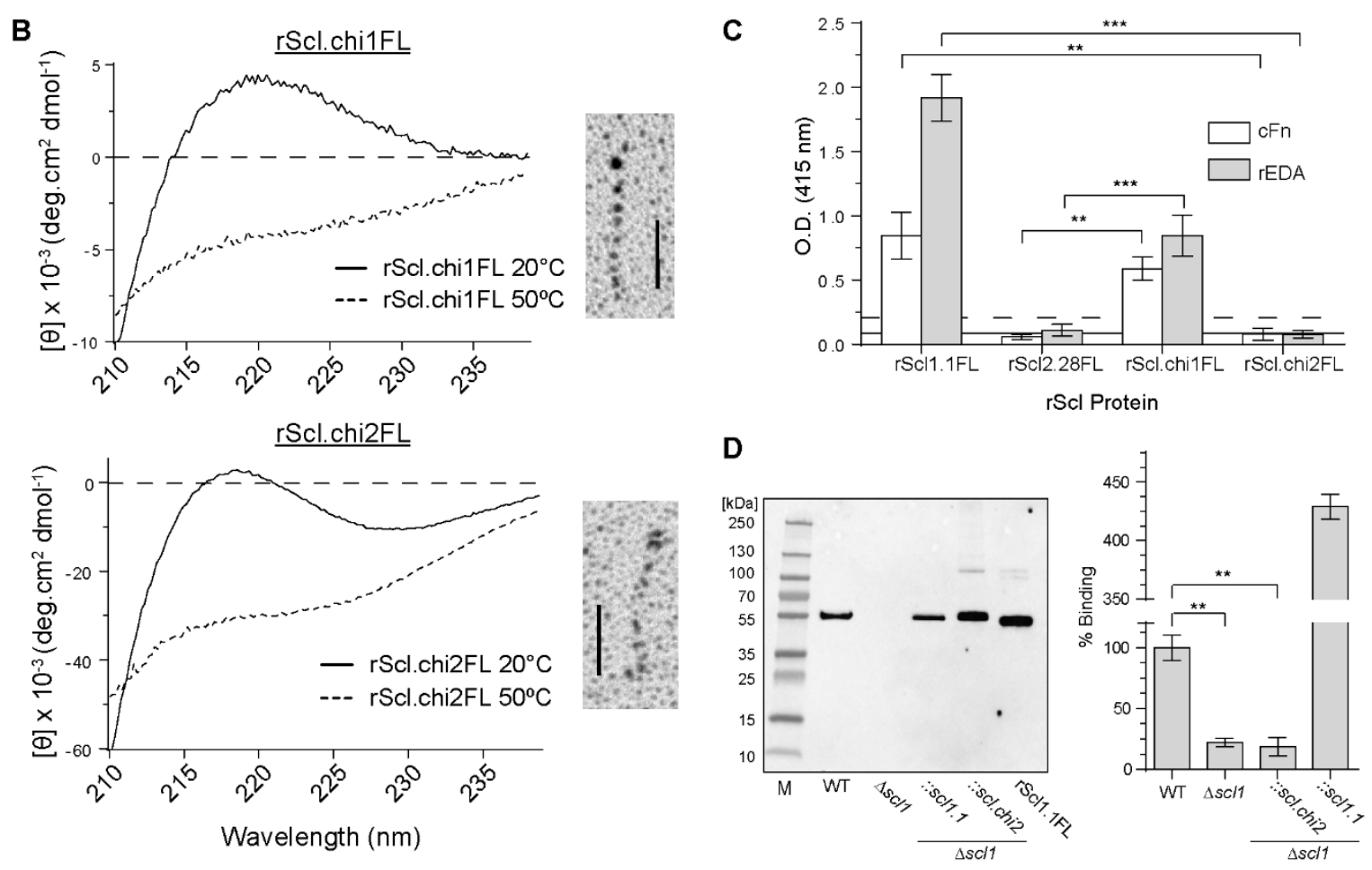

Figure 3. Surface-exposed loop of the Scl1.1-V domain is central in ECM recognition and binding. Full-length chimeric rScl proteins were generated by exchanging V-region loop sequences between ECMbinding-positive and binding-negative rScl proteins; the resulting chimeric rScl.chiFL constructs were characterized structurally by circular dichroism spectroscopy and electron microscopy, as well as functionally for ECM binding. (A) Amino acid sequences of the $\mathrm{V}$ domains of native (rScl1.1FL and rScl2.28FL) and chimeric ( $\mathrm{rScl}$. chi1FL and $\mathrm{rScl} . \mathrm{Fh} 2 \mathrm{FL}$ ) proteins. rScl.chi1FL resulted from the insertion of 22-amino-acids from the ECM-binding-positive variant $\mathrm{rScl1}$.1FL in place of the corresponding loop region of the binding-negative variant $\mathrm{rScl} 2.28 \mathrm{FL}$, whereas $\mathrm{rScl} . \mathrm{chi} 2 \mathrm{FL}$ resulted from the reverse replacement; inserted sequences are shown in italics and numbers mark residue number within V-domain. $(B)$ Structural characterization of the chimeric rScl.chiFL constructs. Left, CD wavelength scans of folded $\left(20^{\circ} \mathrm{C}\right.$, solid 
lines) or denatured $\left(50^{\circ} \mathrm{C}\right.$, dashed lines) rScl.chiFL proteins are shown. Right, structural organization viewed by electron microscopy of the rotary shadowed rScl.chi1FL and rScl.chi2FL shows characteristic two-domain organization; Bars, $50 \mathrm{~nm}$. (C) ECM binding to full-length original and chimeric rScl constructs by ELISA. rScl proteins were immobilized onto Strep-Tactin-coated microplate wells and incubated with ECM ligands, cellular fibronectin ( $\mathrm{CFn}$ ) and recombinant extra domain A (rEDA). Primary anti-ECM Abs and HRP-conjugated secondary Abs were used for ligand detection. Graph bars indicate the mean $\mathrm{OD}_{415} \mathrm{~nm}$ normalized against BSA controls. Statistical analysis was calculated using Student's two-tailed $t$-test, from three independent experiments, each performed in triplicate wells $(\mathrm{N}=3 \pm \mathrm{SD}) ;{ }^{* *} P \leq 0.01,{ }^{* * *} P \leq 0.001$. Statistical significance evaluates the differences between ECM binding by chimeric proteins $r S c l . c h i 1 F L$ and rScl.chi2FL, and the control proteins, rScl2.28FL and rScl1.1FL. Solid (rEDA) and dashed (cFn) lines indicate threshold $\mathrm{OD}_{415} \mathrm{~nm} \pm 2 \mathrm{SD}$ values recorded for experimentally validated ECM-binding-negative rScl2.28FL control protein. (D) rEDA binding to whole GAS cells. M1-type GAS wild-type (WT), isogenic $s c / 1$ inactivated mutant $(\Delta s c / 1)$, and mutant complemented for the expression of Scl1.1 ( $\Delta s c / 1:: s c / 1.1)$ and Scl.chi2 ( $\Delta s c 11:: s c l . c h i 2)$ proteins are shown. Left, Western blot detection of Scl1 expression in cell wall fractions. $25 \mu \mathrm{Ls}$ of cell wall samples prepared from GAS WT and $\Delta s c / 1$, , and $5 \mu \mathrm{Ls}$ of samples from complemented $\Delta s c / 1:: s c / 1.1$ and $\Delta s c / 1:: s c l . c h i 2$ cells overexpressing Scl proteins, were loaded per well. rScl1.1FL protein was included as a control. Scl1 was detected with anti-Scl1.1-V antibody. Right, rEDA binding to whole-GAS cells by flow cytometry. Cells were incubated with rEDA, and binding was detected with primary anti-his-tag $\mathrm{mAb}$ and secondary Ab conjugated to Alexa Fluor ${ }^{\circledR} 568$. Binding to WT cells was set as $100 \%$. Statistical analysis was calculated using Student's two-tailed $t$-test from three independent experiments $(\mathrm{N}=3 \pm \mathrm{SD}) ;{ }^{*} P \leq 0.01$. 


\section{Figure 4}

A

rScl1.1

${ }^{1}$ EVSSTTMTSSQRESKIKEIEFSLK ${ }^{24}$ - ${ }^{25}$ KYPEVSNEKFWERKWYGTYFKE ${ }^{46}$ - ${ }^{47}$ EDFQKELKDFTEKRLKEILDLIGKSKIK ${ }^{74}$ Helix1

Loop

Helix 2

rScl2.28

${ }^{1}$ DEQEEKAKVRTELIQELAQ ${ }^{19}$ - ${ }^{20}$ GLGGIEKKNFP TLGDEDLDHTYMTKL ${ }^{45}$ - ${ }^{46}$ LTYLQEREQAENSWRKRLLKGIQDHALD $^{73}$

Helix1 rScl.chiN

rScl.chiN
'DEQEEKAKVRTELIQELAQ ${ }^{19}{ }_{-}^{20}$ KYPEVSNEKFWTLGDEDLDHTYLTY ${ }^{42}-{ }^{43}$ LQEREQAENSWRKRLLKGIQDHALD $^{70}$

Helix1

Loop

Helix 2

${ }^{1}$ DEQEEKAKVRTELIQELAQ ${ }^{19}{ }^{20} \mathrm{GLGGIEKKNFPERKWY}$

Helix1

Loop

Helix 2

rScl.chil

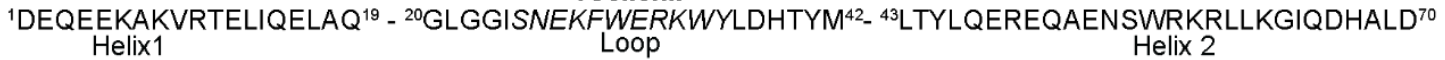

B
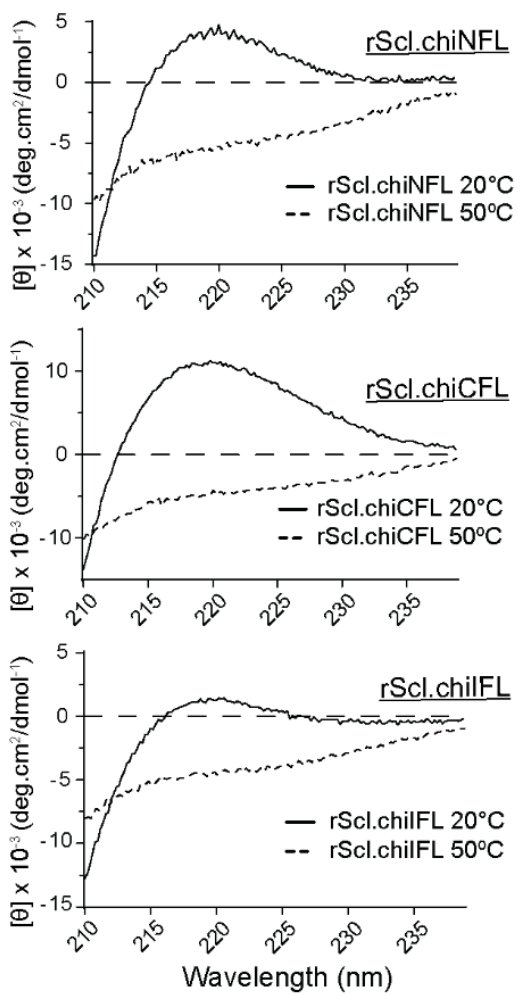

C
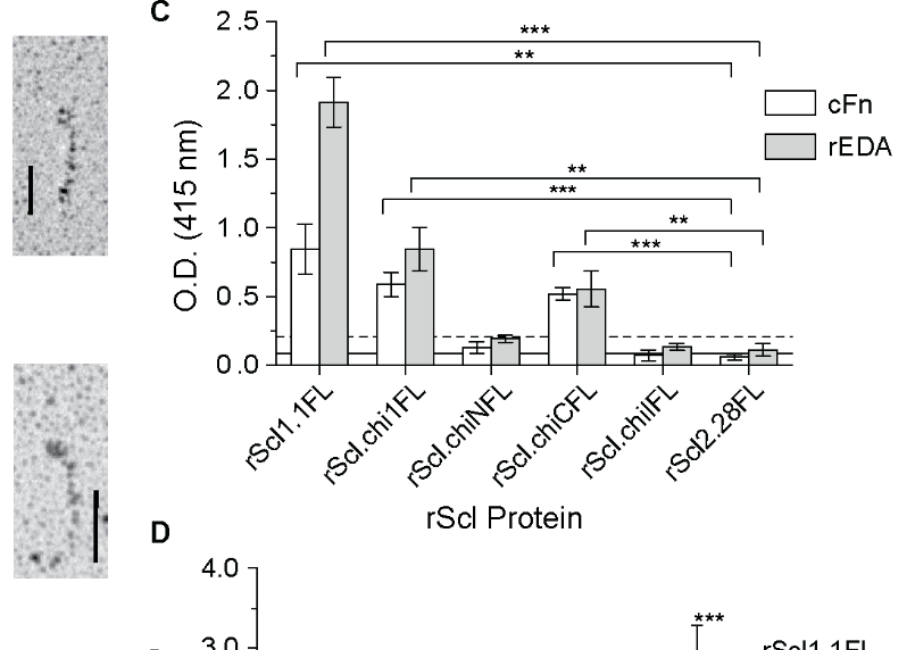

D
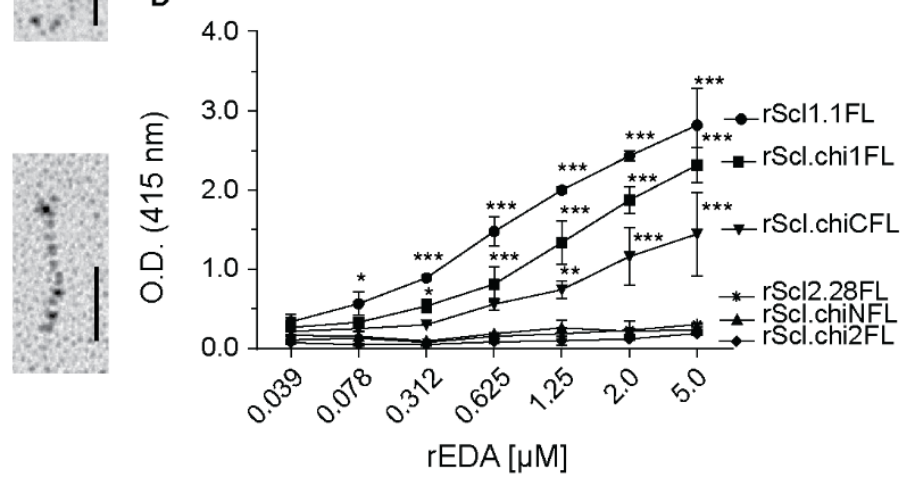

Figure 4. The C-terminal 11 amino acids of the loop segment of the Scl1.1-V domain is central in ECM recognition and binding. Chimeric $\mathrm{rScl}$ proteins were generated by exchanging portions of the loop from ECM-binding-positive rScl1.1-V with respective loop sequences of the binding-negative protein rScl2.28FL; the resulting chimeric rScl.chi constructs were characterized structurally by circular dichroism spectroscopy and electron microscopy, as well as functionally for ECM binding by ELISA. $(A)$ Amino acid sequences of the $\mathrm{V}$ domains of native (rScl1.1FL and rScl2.28FL) and chimeric ( $r S c l . c h i N F L, r S c l . c h i C F L$, rScl.chilFL) proteins. rScl.chiNFL resulted from the insertion of the N-terminal 11 amino acids of the rScl1.1FL-loop sequence in place of the corresponding $\mathrm{N}$-terminal loop region of the rScl2.28FL, whereas 
rScl.chiCFL resulted from the replacement of the C-terminal 11 amino acids and rScl.chilFL from the replacement of internal 11 amino acids; inserted sequences are shown in italics and numbers mark residue number within V-domain. (B) Structural characterization of the chimeric rScl constructs. Left, CD wavelength scans of folded $\left(20^{\circ} \mathrm{C}\right.$, solid lines) or denatured $\left(50^{\circ} \mathrm{C}\right.$, dashed lines) rScl.chi proteins are shown. Right, structural organization viewed by electron microscopy of the rotary shadowed rScl.chiNFL, rScl.chiCFL, and rScl.chilFL shows characteristic two-domain organization; Bars, $50 \mathrm{~nm}$. (C) ECM binding to chimeric $\mathrm{rScl}$ constructs by ELISA. rScl proteins were immobilized onto Strep-Tactin-coated microplate wells and incubated with ECM ligands, cellular fibronectin (cFn) and recombinant extra domain A ( $r E D A)$. Primary anti-ECM Abs and HRP-conjugated secondary Abs were used for ligand detection. Graphic bars indicate the mean $\mathrm{OD}_{415} \mathrm{~nm}$ normalized against BSA controls. Statistical analysis was calculated using Student's two-tailed $t$-test, from three independent experiments, each performed in triplicate wells ( $N=3 \pm$ $\mathrm{SD}) ;{ }^{* *} P \leq 0.01,{ }^{* * *} P \leq 0.001$. Solid (rEDA) and dashed (cFn) lines indicate threshold $\mathrm{OD}_{415} \mathrm{~nm} \pm 2 \mathrm{SD}$ values recorded for experimentally validated binding-negative $\mathrm{rScl} 2.28 \mathrm{FL}$ control protein. $(D)$ Concentrationdependent binding of rEDA to rScl constructs. rScl proteins were immobilized onto Strep-Tactin-coated microplate wells and incubated with increasing concentrations of rEDA. Wells were further developed, as described above. Statistical analysis was calculated using two-way ANOVA, from two-independent experiments, each performed in triplicate wells ( $\mathrm{N}=2 \pm \mathrm{SD}$ ), ${ }^{*} P \leq 0.05,{ }^{* *} P \leq 0.01,{ }^{* * *} P \leq 0.001$. Statistical significance evaluates difference between bindings of each rScl1-chimeric protein to binding-negative control rScl2.28FL. 
Figure 5

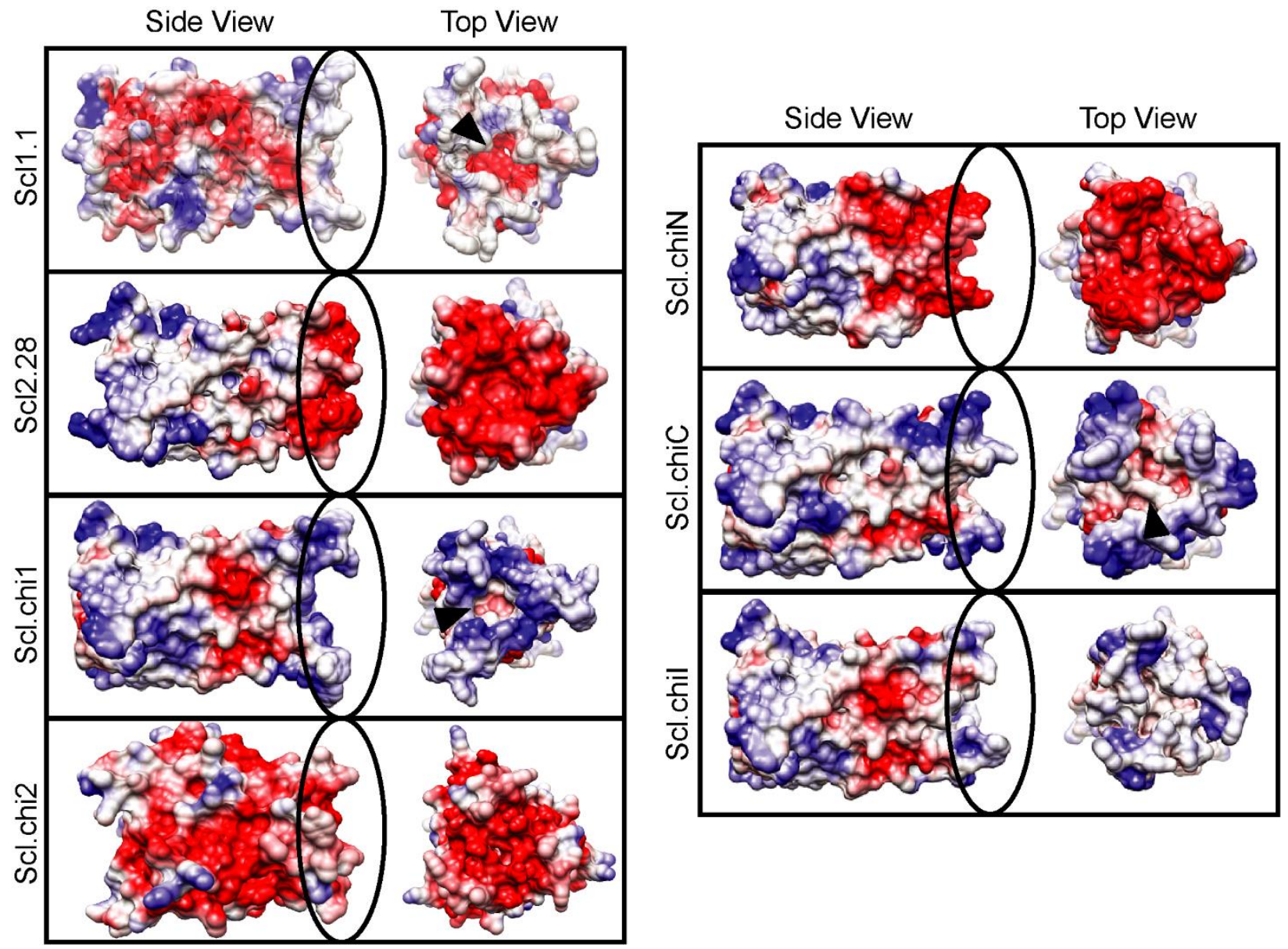

Figure 5. Electrostatic potential surfaces of Scl1-V, Scl2-V, and Scl.chi-V domains. The positive and negative charges are colored blue and red, respectively. Exposed loops are outlined by the black ovals in side views. Black arrowheads point to negatively charged pockets of the $D / E$ residues, embedded in neutral and positively-charged environment, seen within the binding-positive Scl1.1-V and Scl.chiC-V domains. Homology models were generated using the crystal structure of the Scl2.3 V domain (PDB accession code$4 \mathrm{nsm})$, as a template. 


\section{Figure 6}

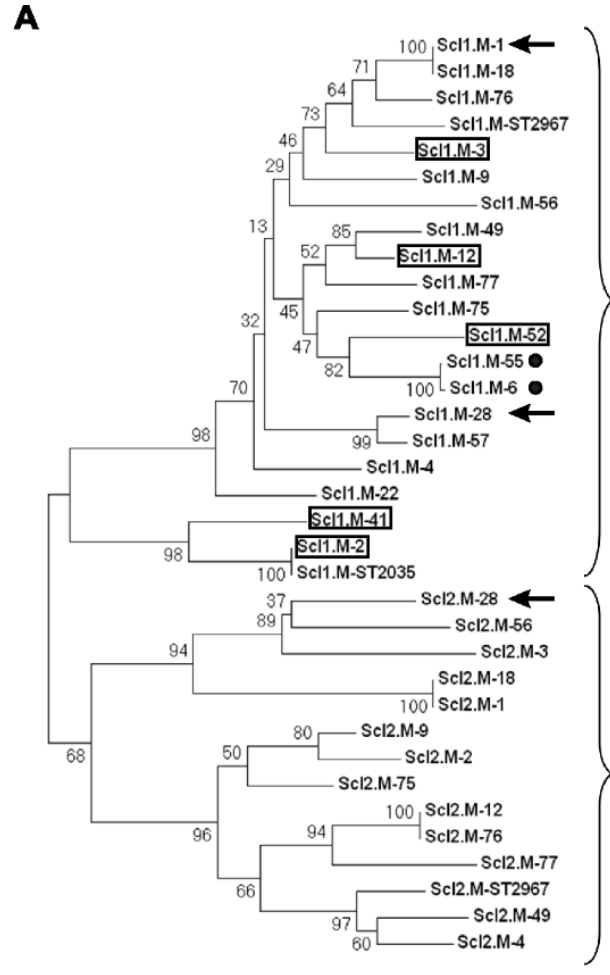

B

EVSTAAMTKEERQQEIEKIKEDIK24 - ${ }^{25}$ KEYPKASEEKFWESSFWGRRYFNE ${ }^{48}$ - ${ }^{49}$ QEYLKSLQSFTEQRLQDIVNLIEKGATK ${ }^{76}$ Helix 1
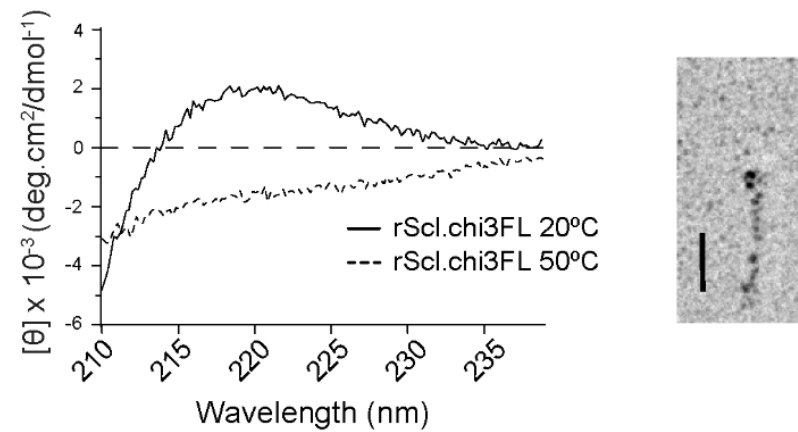

D

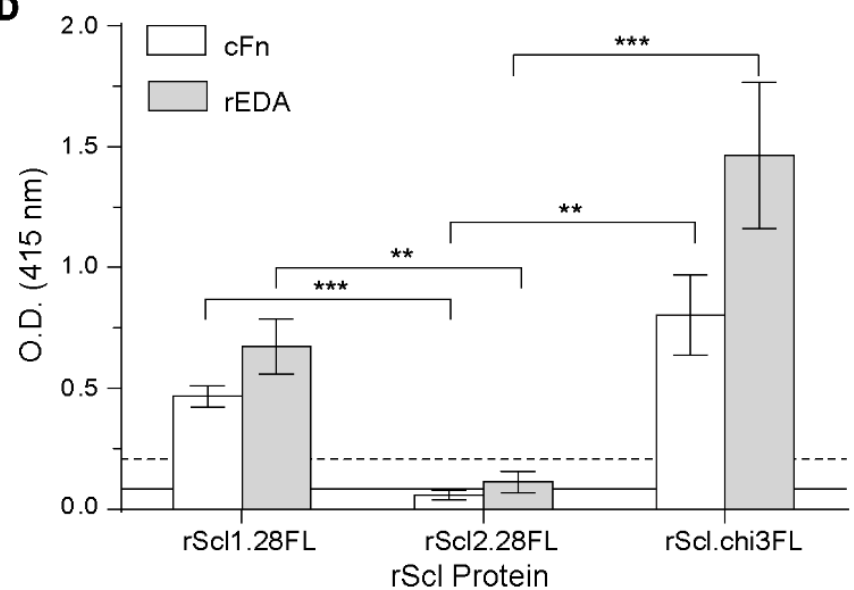

Helix 2

rScl2.28

1DEQEEKAKVRTELIQELAQ ${ }^{19}$ - ${ }^{20}$ GLGGOEKKNFPTLGDEDLDHTYMTKL 45 - ${ }^{46}$ LTYLQEREQAENSWRKRLLKGIQDHALD73 Helix 1

Helix 2

rscl.chi3

${ }^{1}$ DEQEEKAKVRTELIQELAQ ${ }^{19}$ ـ ${ }^{20}$ KEYPKASEEKFWESSFWGRRYFNE ${ }^{43}$ - ${ }^{44}$ LTYLQEREQAENSWRKRLLKGIQDHALD $^{71}$ Helix 1

Figure 6. Conservation of ECM recognition and binding by phylogenetically distant Scl1 variant. $(A)$ Phylogenetic tree of Scl1-V and Scl2-V regions. Branch lengths are scaled to genetic distance and bootstrap values are shown for all nodes. Scl1 variants with known ECM-binding capacities are boxed, while variants known to bind complement components, Factor $\mathrm{H}$ and Factor $\mathrm{H}$-related protein 1, are marked with dots. ECM-binding-positive variants, Scl1.28 and Scl1.1, and binding-negative variant Scl2.28, used for loop switching, are marked by arrows. (B) Amino acid sequences of the V-domains of native (rScl1.28FL and $\mathrm{rScl} 2.28 \mathrm{FL}$ ) and chimeric ( $\mathrm{rScl} . \mathrm{chi3FL}$ ) proteins. rScl.chi3FL resulted from the insertion of the 22amino-acids from the ECM-binding-positive variant rScl1.28FL in place of the corresponding loop-region of the binding-negative variant rScl2.28FL; inserted sequence is italicized and numbers mark residues within V-domain. (C) Structural characterization of rScl.chi3FL. Left, CD wavelength scans of folded (20C, solid 
line) or denatured $\left(50^{\circ} \mathrm{C}\right.$, dashed line) rScl.chi3FL protein are shown. Right, structural organization viewed by electron microscopy of the rotary shadowed $\mathrm{rScl}$.chi3FL shows characteristic two-domain organization; Bar, $50 \mathrm{~nm}$. (D) ECM binding to rScl.chi3FL by ELISA. rScl proteins were immobilized onto Strep-Tactincoated microplate wells and incubated with ECM ligands, cellular fibronectin (cFn) and recombinant extra domain A (rEDA). Primary anti-ECM Abs and HRP-conjugated secondary Abs were used for ligand detection. Graphic bars indicate the mean $\mathrm{OD}_{415} \mathrm{~nm}$ normalized against BSA controls. Statistical analysis was calculated using Student's two-tailed $t$-test, from three independent experiments, each performed in triplicate wells $(\mathrm{N}=3 \pm \mathrm{SD}) ;{ }^{* *} P \leq 0.01,{ }^{* * *} P \leq 0.001$. Solid (rEDA) and dashed (cFn) lines indicate threshold $\mathrm{OD}_{415} \mathrm{~nm} \pm 2 \mathrm{SD}$ values recorded for experimentally validated $\mathrm{ECM}$-binding-negative rScl2.28FL control protein. 


\section{Figure 7}

A

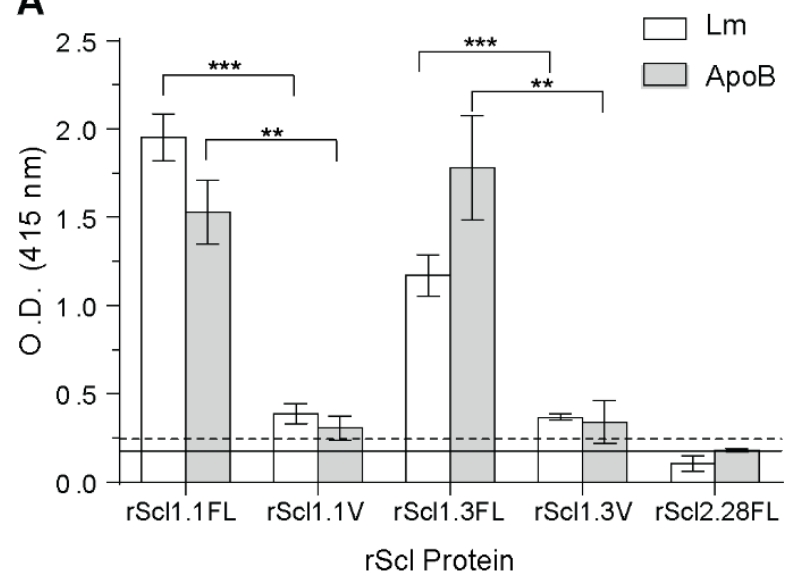

C

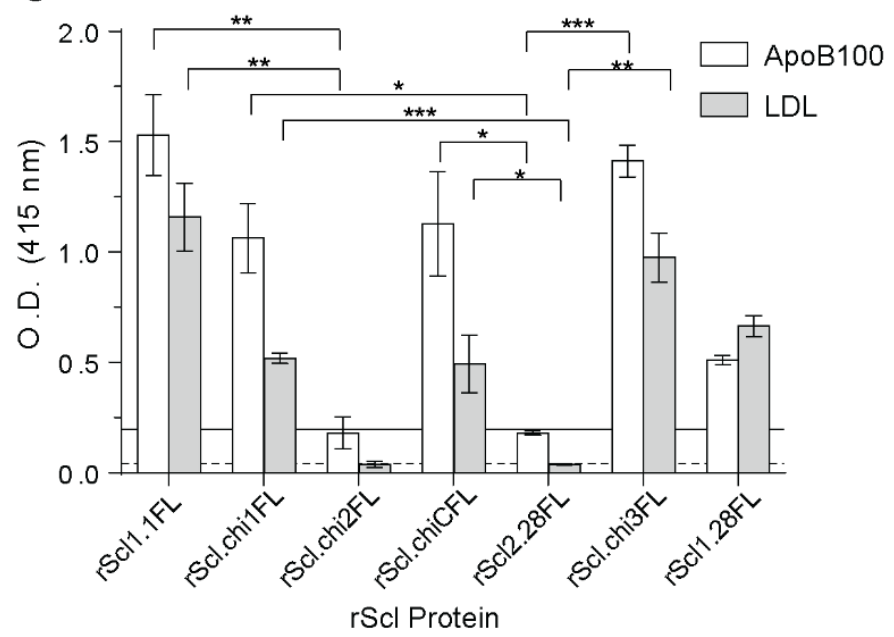

B

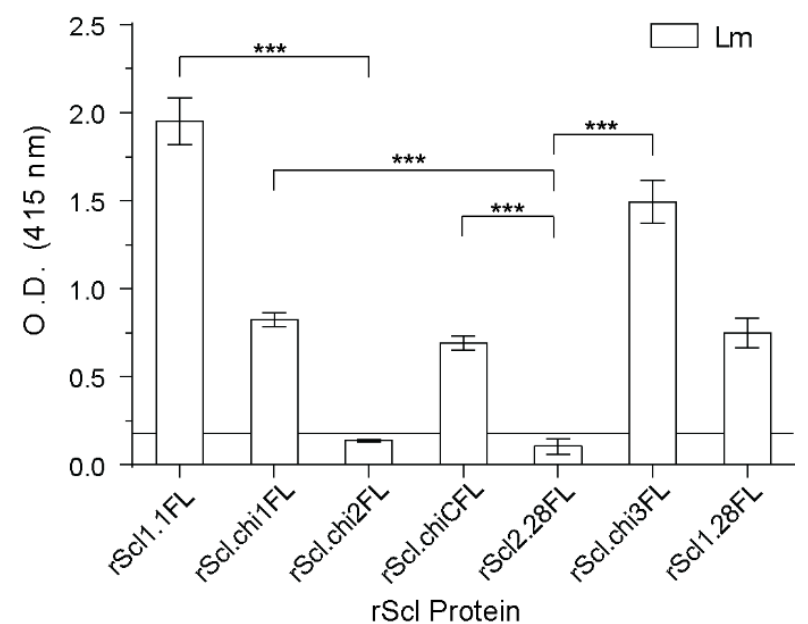

Figure 7. Conserved structural constraints and exposed loop of Scl1 proteins determine binding to laminin and apolipoprotein B100/low-density lipoprotein. Full-length recombinant Scl1 proteins, chimeric rScl proteins, and rScl1-V-only constructs were tested for binding to laminin (Lm), and apolipoprotein B100 (ApoB100) and low-density lipoprotein (LDL). rScl proteins were immobilized onto Strep-Tactin-coated microplate wells and incubated with ligands. Primary anti-Lm or anti-ApoB100 Abs and HRP-conjugated secondary Abs were used for ligand detection. Graphic bars indicate the mean OD $415 \mathrm{~nm}$ normalized against BSA-coated control wells. Statistical analysis was calculated using Student's two-tailed $t$-test from three independent experiments, each performed in triplicate wells $(\mathrm{N}=3 \pm \mathrm{SD}) ;{ }^{*} P \leq 0.05$, ${ }^{* *} P$ $\leq 0.01,{ }^{* * *} P \leq 0.001$. (A) Full-length Scl1 proteins are required for binding to $\mathrm{Lm}$ and ApoB100. Statistical significance evaluates the differences between binding by the full-length proteins, rScl1.1FL and rScl1.3FL, and their respective V-region only constructs, rScl1.1V and rScl1.3V. Dashed line (ApoB) and solid line 
(Lm) indicate threshold $\mathrm{OD}_{415} \mathrm{~nm} \pm 2 \mathrm{SD}$ values recorded for experimentally validated binding-negative control protein rScl2.28FL. $(B, C)$ Surface-exposed loop of the Scl1-V domain is central in $\mathrm{Lm}(B)$ and ApoB100/LDL $(C)$ recognition and binding. Dashed (LDL) and solid lines ( $B-L m, C-A p o B)$ indicate threshold $\mathrm{OD}_{415} \mathrm{~nm} \pm 2 S \mathrm{SD}$ values recorded for experimentally validated binding-negative rScl2.28FL control protein. 


\title{
CHAPTER 2
}

\author{
ADAPTATION OF THE GROUP A STREPTOCOCCUS ADHESIN SCL1 TO BIND \\ FIBRONECTIN TYPE III REPEATS WITHIN WOUND-ASSOCIATED \\ EXTRACELLULAR MATRIX: IMPLICATIONS IN CANCER TARGETING \\ Dudley H. McNitt, Soo Jeon Choi, Jessica L. Allen, River A. Hames, Scott A. Weed, \\ Livingston Van De Water, Rita Berisio, Slawomir Lukomski \\ Submitted in 2019 to Molecular Microbiology - Under review
}

\begin{abstract}
The human-adapted pathogen group A Streptococcus (GAS) utilizes wounds as a portal of entry into host tissues, wherein surface adhesins interact with the extracellular matrix, enabling bacterial colonization. The streptococcal collagen-like protein 1 (Scl1) is a major adhesin of GAS, which selectively binds to fibronectin type III (FnIII) repeats, extra domains $A$ and $B$ in cellular fibronectin and to several Fnlll repeats within tenascin-C. Binding to Fnlll repeats occurred through a conserved mechanism, involving the Scl1 globular domain, and facilitated GAS adherence and biofilm formation. Numerous extracellular matrix proteins can be targeted by group A streptococcal adhesins to enter the human host. The extra domains $A$ and $B$ are unique in that they are alternatively spliced in cellular fibronectin and appear transiently in the wound extracellular matrix. This Scl1-Fnlll binding is therefore a remarkable example of GAS adaptation to this transient environment that is initially encountered within the host. Cellular fibronectin isoforms and tenascin- $\mathrm{C}$ are also present in the tumor microenvironment, and here, we found that Scl1 mediated GAS attachment and biofilm formation on the extracellular matrices deposited by cancer-associated fibroblasts and osteosarcoma cells. These data lay the groundwork for the assessment of Scl1 as a cancer-targeting biomolecule.
\end{abstract}

\section{INTRODUCTION}

Group A Streptococcus (GAS or Streptococcus pyogenes) is an obligate human pathogen that is responsible for over 700 million infections worldwide each year (1). Infection by GAS can result in diseases that range in severity, from highly prevalent superficial infections to fatal conditions (1). GAS infection can also lead to the development of postinfectious autoimmune sequelae (2-4). Invasive infections and rheumatic heart diseases 
results in over 500,000 deaths globally each year $(1,5)$, which places GAS among the top ten most lethal infectious disease-causing organisms (5). Nevertheless, superficial GAS infections account for the vast majority ( 95.5\%) of the 700 million infections, mostly affecting children; GAS also asymptomatically colonizes the throat and skin of $5-25 \%$ of children of the general population (6). GAS isolates are subtyped based on the 5'hypervariable end of the emm gene, encoding the N-terminus of the M-protein, a major surface adhesin and virulence factor of GAS (7). There have been over 220 different emm types of GAS identified and there is a non-random correlation between certain M-types and infection-associated diseases (8).

In addition to protein M, GAS express numerous adhesins that contribute to host colonization (9). Two ubiquitous surface-associated proteins are the streptococcal collagen-like protein 1 (Scl1/SclA) and 2 (Scl2/SclB) (10-14). Scl1 and Scl2 share a unique structure that is distinct from other GAS adhesins; both are homotrimeric and have similar "Iollipop-like" structural organization $(15,16)$. The N-terminal sequence-variable (V) globular domain is followed by the collagen-like (CL) domain, and with a LPATG cellwall anchoring motif at the C-terminus $(11,12)$. The V-domain of the Scl2 protein from M3type GAS has been crystalized (17). It forms a six-helix bundle, where two antiparallel $\alpha$ helices in each monomer are joined by surface-exposed loops. $(18,19)$. Regardless of the conserved structure, the amino-acid sequences of the V-domains diverge between Scl1 and Scl2 proteins and also differ among Scl1 and Scl2 variants from strains of different M-types (20). The Scl1-V domain binds selected isoforms of cellular fibronectin (cFn) that are expressed within wounded tissue $(21,22)$, via the surface-exposed loops of the $V$ domain (19).

cFn is a high molecular weight glycoprotein encoded by the FN1 gene that contains three different types of repeating domains (I, II, and III) $(23,24)$. Type I and II repeats interact with fibrin, fibronectin and collagen, while the type III repeats contain numerous cell-binding sites via integrins and other receptors (25). There are over 20 different isoforms of cFn in humans due to alternative splicing of FN1-mRNA, which can lead to the inclusion of extra domains. These extra domains are both fibronectin type III (FnIII) repeats, known as extra domain $A(E D A / E I I I A)$ and extra domain $B(E D B / E I I I B)(26)$. The EDA and EDB repeats vary in sequence but retain the conserved prototypical FnIII-repeat 
$\beta$-sandwich structure $(27,28)$. Spatial and temporal inclusion of EDA and EDB domains in cFn is tightly regulated; both are expressed during embryogenesis but are not readily detectable in healthy adult tissue $(29,30)$. Conversely, isoforms of cFn that contain EDA and/or EDB are expressed in pathological adult tissues, such as within wound beds and around tumors (31-33). EDA has been shown to play important roles in wound healing $(34,35)$. The role of EDB in the wound microenvironment is not well understood, but it is known to be highly expressed during angiogenesis of healing tissues and of tumors (3638). Tenascin-C (TnC) is another ECM protein that contains canonical Fnlll repeats (39). Like EDA and EDB of cFn, $\mathrm{TnC}$ is predominately expressed during fetal development and is found at negligible levels within normal adult soft tissue (40-42); though, it is highly deposited in wound and within tumor stroma $(39,43)$.

We previously reported that Scl1 binds to the wound-associated fibronectin type III repeat, EDA, and this binding contributes to GAS colonization and biofilm formation on matrices deposited by normal human dermal fibroblasts (22). In this report, we show that Scl1 binds to recombinant EDB and to some Fnlll repeats within TnC. Binding was conserved across phylogenetically distant Scl1 variants, originating from strains of epidemiologically relevant $M$ types. Recognition of both ligands was mediated through the Scl1-V-domain loop-region, the same binding mechanism as for the EDA segment within cFn (19). Scl1 facilitates GAS biofilm formation on rEDB coatings. In addition, ECM deposited by cancer-associated fibroblasts and osteosarcoma cells contains cFn isoforms with EDA and/or EDB, and TnC, capable of binding Scl1-expressing GAS to facilitate biofilm formation. This work identifies novel wound-associated ECM targets for Scl1 that are expressed at the portal of entry for GAS and facilitate host colonization at wound sites. The unique binding of Scl1 to EDA, EDB and TnC and may present alternative opportunities to target tumors or other disease regions that selectively express these ECM variants.

\section{RESULTS}

Scl1 binds to EDB of cFn via surface exposed loops - Previously, we established that Scl1 binds to cFn, but not plasma fibronectin (pFn) (21), via the C-C' loop of EDA (22). In addition to EDA, cFn can include another extra domain, EDB, which is also upregulated 
within wounded tissue (31). EDA and EDB both adopt a typical fibronectin type III repeat structure $(27,28)$, comprised of $7 \beta$-strands $(A, B, C, C, E, F, G)$ that form $2 \beta$-sheets, with strands connected by flexible loops $(27,28)$, including the C-C' loop that mediates Scl1 binding to EDA (Fig. 1A). Interestingly, we determined that the commercial cFn preparations we used in binding assays contained cFns that included the EDB segment (data not shown). Therefore, we hypothesized that Scl1 may also bind to EDB by a similar mechanism, involving the surface-exposed loops of Scl1-V domain (19).

To test our hypothesis, we devised a panel of chimeric $\mathrm{rScl}$ constructs to be used in binding assays with rEDB protein (Fig. 1B). A chimeric rScl construct was devised, wherein we replaced the loop region (22 amino acids) of the EDA-binding-negative rScl2.28-V domain, originating from Scl2 of M28-type strain, with that originating from EDA-binding-positive rScl1.1 (Scl1 of M1 strain), resulting in rScl.chimera1 (rScl.chi1). We correspondingly did the reverse, replacing the loop-region from rScl1.1-V domain with the loop from rScl2.28-V domain, resulting in rScl.chi2. We also devised the rScl.chiC construct, with a partial loop substitution (11 amino acids) from Scl1.1 that replaced an analogous loop sequence of rScl2.28; this loop fragment was responsible for EDA recognition. To assess whether this EDB-binding mechanism is true for a different Scl1 variant, we similarly replaced the whole loop (22 aa) with another EDA-binding-positive sequence from a phylogenetically distant Scl1.28 protein, resulting in rScl.chi3. This panel of devised $\mathrm{rScl}$ constructs was next tested for rEDB binding and compared to our previously reported binding data with rEDA (19).

Both naturally-derived rScl1.1, rScl1.3, rScl1.28 and rScl1.41, as well as the chimeric proteins rScl.chi1-3 and $\mathrm{rScl}$.chiC were tested for binding to rEDB by ELISA; binding was compared to that of rEDA-binding-negative rScl2.28 control. As it was previously established for rEDA binding, all rScl1 proteins, derived from Scl1 variants originating from several GAS M-types (M1, M3, M28 and M41), bind rEDB to varying degrees, while control rScl2.28 protein did not bind (Fig. 1C). We furthermore demonstrate that the Scl1-V loop mediates rEDB binding, as evidenced by rEDB binding to the chimeric constructs rScl.chi1, rScl.chi3, and rScl.chiC, and a loss of binding to rScl.chi2, harboring a reverse loop substitution. These data reveal that the Scl1 adhesin binds to both the EDA and EDB segments that are found within cFn, expressed in the 
wounded portal of entry, and signify the importance of the Scl1-V loop in EDB/EDA recognition and binding.

Scl1 mediates GAS-EDB binding and promotes biofilm formation - We next investigated if Scl1 expressed on GAS cells mediates EDB binding, using flow cytometry (Fig. 1D). Deposition of rEDB onto the surface of M1 and M41 strains was assessed after 30-minute incubation. M1 GAS is isolated across the globe and is responsible for both superficial and invasive infections, while M41 is historically associated with superficial skin infections $(5,44)$ The rEDB binding to GAS WT cells was compared to that measured for the corresponding isogenic Scl1-deficient mutant cells, M1 $\Delta \mathrm{s} / 1$ and M41 $1 \Delta \mathrm{s} / 1$. We also tested rEDB binding to the M1 $1 \Delta \mathrm{s} / 1$ mutant cells complemented in trans for the expression of either the original Scl1.1 protein $(\Delta s c / 1:: s c / 1.1)$ or the chimeric Scl.chi2 variant ( $\triangle \mathrm{sc} / 1:: \mathrm{scl}$.chi2). There was a significant reduction in rEDB deposition on the surface of both isogenic $M 1 \Delta s c / 1$ and $M 41 \Delta s c / 1$ mutants, compared to their respective WT strains. In addition, restoration of Scl1.1 or Scl.chi2 expression on the surface of the M1 $\Delta s c / 1$ mutant restored rEDB binding (Fig. 1D). Thus, just like with rScl1-rEDB binding above, our data demonstrate for the first time that GAS can bind to EDB containing cFn isoforms through surface Scl1.

Because wounds, which contain isoforms of cFn that contain either EDA and/or EDB fibronectin, can serve as a portal of entry for GAS to gain access to host tissues, we sought to understand if the GAS-EDB interaction facilitates GAS biofilm formation in vitro, which could influence the course of GAS infection. We used rEDB-coated surfaces and analyzed biofilm formation by GAS isogenic strains spectrophotometrically, using crystal violet staining to assess biomass (Fig. 1E), as well as microscopically, with GFPexpressing M41 GAS, to examine biofilm morphology and thickness (Fig. 1F). After 24 hours, there was significantly more stained biomass of WT strains grown on rEDB, compared to amounts of biomass of the isogenic M1 $1 \Delta \mathrm{sc} / 1$ and $\mathrm{M} 41 \Delta \mathrm{sc} / 1$ mutants (Fig. $1 E)$. These results were further supported through confocal laser scanning microscopy, demonstrating that M41 WT biofilm had an average thickness of approximately $18 \mu \mathrm{m}$, while the M41 $\Delta s c / 1$ mutant had an average thickness of approximately $8 \mu \mathrm{m}$, a $40 \%$ 
decrease (Fig. 1F, bottom images). Altogether, our data validate GAS capacity for binding to the fibronectin type III repeat, EDB, which facilitates GAS biofilm formation.

Scl1 binds tenascin C via surface-exposed loops of the Scl1-V domain - TnC is a multi-domain glycoprotein with a central region made of fibronectin type III repeats (Fig. 2A) (45). Similar to EDA- and EDB-fibronectins, TnC is abundantly deposited within wounded tissue $(39,46)$. Here, we hypothesized that Scl1 could bind to TnC. We first investigated $\mathrm{TnC}$ binding to our panel of $\mathrm{rScl} 1$ and $\mathrm{rScl} 2$ constructs that we characterized previously for binding to cFn (21) and rEDB above (Fig. 1B-C). ELISA binding data demonstrate that constructs rScl1.1, rScl1.2, rScl1.12, rScl1.28 and rScl1.41, derived from diverse Scl1 variants expressed by M1-, M2-, M12, M28 and M41 GAS, all bind to TnC. In contrast, rScl2.4 (Scl2 of M4) and rScl2.28 (Scl2 of M28) constructs demonstrate minimal levels of $\mathrm{TnC}$ binding (Fig. 2B) and were considered binding-negative controls, reminiscent of their lack of binding to $\mathrm{cFn}, \mathrm{rEDA}$ and $\mathrm{rEDB}$. Based on these results we asked if $\mathrm{TnC}$ binding occurs via the loop-region of the Scl1-V domain, utilizing the same panel of chimeric rScl proteins described above. ELISA binding data demonstrate TnC binding to rScl.chi1, rScl.chiC and rScl.chi3, whereas constructs rScl.chi2 and rScl2.28 are binding-negative (Fig. 2C). Hence, our results establish the same binding pattern documented earlier for EDA of cFn and EDB of cFn, pointing towards one conserved Scl1 binding mechanism to $\mathrm{cFn}$ and $\mathrm{TnC}$, which is mediated by the surface-exposed loops present in the structure of Scl1-V domain $(18,19)$.

Scl1 binds to the type III repeats of tenascin $C$ - We next tested whether Scl1 binds to FnIII repeats within TnC. EDA in cFn interacts with Scl1 (22) and with the $\alpha_{9} \beta_{1}$ integrin receptor (47), which also binds to the TnFn3 repeat in $\mathrm{TnC}$ (48). Therefore, we hypothesized that Scl1 specifically binds to the TnFn3 repeat within the $\mathrm{TnC}$ molecule (Fig. 2D). To test this hypothesis, we produced recombinant fragments of the TnFnIII repeats, encompassing repeats 1-5 (rTnFn1-5), repeat number $3(\mathrm{rTnFn} 3)$, and repeats 6-8 ( $r T n F n 6-8)$, the later to be used as a binding-negative control. We first tested the rTnFnlll constructs for binding to our panel of naturally-derived and chimeric $\mathrm{rScl}$ constructs (Fig. 2E). Binding to rTnFn1-5 was significantly higher for all naturally-derived 
rScl1.1, rScl1.28 and rScl1.41 proteins and the chimeric EDA/EDB/TnC-binding-positive proteins rScl.chi1, rScl.chiC, and rScl.chi3, as compared to ECM-binding-negative rScl2.28 and rScl.chi2 controls. However, binding between rTnFn3 and rScl constructs that bind the larger rTnFn1-5 fragment was negligible and inconsistent. Unexpectedly, we also recorded a significant binding between control rTnFn6-8 fragment and $\mathrm{rScl}$ constructs positive for rTnFn1-5 binding (48). In summary, we have demonstrated for the first-time direct binding between Scl1 and TnFnlll repeats using recombinant proteins.

The $\alpha_{9} \beta_{1}$ and $\alpha_{4} \beta_{1}$ integrins were historically referred to as "acidic" integrin receptors that recognized LDV or similar tripeptide sequences (49), like an IDG sequence located in the B-C loop of TnFn3 (Fig. 2D). We reasoned that the Scl1 binding site in TnC may overlap with similar LDV-like sequences, particularly located within the exposed loops of the rTnFn1-5 (outside Fn3 repeat) and rTnFn6-8 fragments, and the homology models of the TnFn1-5 and TnFn6-8 fragments (48) identified several candidate sequences within both TnFn1-5 and TnFn6-8 regions (Fig. 2Fi-ii). Specifically, the VDG triplet was identified in the C'-E loop of TnFn6, the LDG sequence in the connecting segment between repeats TnFn6 and 7, and the LDS sequence in the connecting segment between TnFn7 and 8, and VDG sequence in the B-C loop of TnFn8 (Fig. 2Fi). Most of these share a DG-dipeptide with $C$ - $C$ ' loop in EDA that is critical for integrin $\alpha_{9} \beta_{1}$ binding (47). We similarly identified several LDV-like tripeptides within TnFn1-5 fragment e.g., PEG, LDA, IDS, VDV, LDT, and LEP triplets (Fig. 2Fii). We propose that Scl1 may recognize the TnFnllI repeats $1-5$ and $6-8$ via LDV/IDG-like motifs, similar to some the $\alpha_{9} \beta_{1}$ binding sequence (50).

\section{Identification of Scl1 targets within the ECM deposited by cancer-associated} fibroblasts - Tumors have many similarities to healing wounds, including extensive ECM remodeling (51). ECM deposition and remodeling is due in part to the surrounding stromal cancer-associated fibroblasts (CAFs) $(38,52,53)$ that deposit EDA/EDB-fibronectins and TnC (52,54-57). Here, we characterized the extracellular matrix deposited by primary CAFs as an in vitro model for Scl1 targeting to the tumor microenvironment, expressing significant amounts of $\mathrm{cFn}$ isoforms that contain EDA and/or EDB, and TnC. CAFs were grown to confluency and were subsequently removed non-proteolytically from deposited 
matrices by EGTA treatment. Matrices were initially visualized using Ponceau S staining, showing good overall integrity of a complex fibrillary network, without obvious signs of degradation (Fig. 3A). The composition of the matrices was then assayed by ELISA for the presence of cFn, EDA, EDB, and TnC, using specific mAbs (Fig. 3B). ELISA data shows that CAFs deposit ECM that contains 2-7 fold higher levels of cFn with EDA, EDB, as well as $\mathrm{TnC}$, relative to control BSA background. Matrices were also visualized using immunofluorescent staining of the specific distribution of ECM components with the same mAbs, used in the ELISA. Immunofluorescence imaging revealed a significant presence of cFn isoforms that contain EDA and EDB, as well as the presence of TnC within CAFdeposited ECM (Fig. 3C). We conclude that we developed a suitable in vitro model to test Scl1 binding to complex extracellular matrices potentially akin to those generated by fibroblasts in wounds and the tumor microenvironment.

Scl1 mediates GAS targeting to cancer-associated fibroblast-derived ECM-We first visualized and quantified the GFP-expressing WT and isogenic M1 $\Delta s c / 1$ and M41 $\Delta s c / 1$ mutant cells adherence to glass coverslips, containing CAF-deposited matrices, after 1 hour incubation (Fig. 4A). More fluorescent M1- and M41-WT GAS were seen adhered to the CAF-derived ECM on coverslips than their respective $\mathrm{M} 1 \Delta s \mathrm{~s} / 1$ and $\mathrm{M} 41 \Delta \mathrm{sc} / 1$ mutants (Fig. 4A, left). The quantification of attached cells revealed that significantly fewer of the M1 $\Delta s c / 1$ mutant cells, by 70\%, bound the matrices, compared to the WT strain; similarly, the M41 $\Delta$ scl1 mutant bound the matrices $\sim 80 \%$ less than the WT strain (Fig. $4 \mathrm{~A}$, right). Therefore, our results show that GAS adheres to the ECM deposited by CAFs, and this attachment is mediated through Scl1.

We next investigated if Scl1 expression facilitates GAS biofilm formation on the CAF-deposited matrix, using crystal violet staining and confocal fluorescence microscopy (Fig. 4B-C). Crystal violet staining of 24-hour bacterial biomass formed on CAF-derived ECM detected significantly more of both $M 1$ and M41 WT GAS, compared to their respective $\mathrm{M} 1 \Delta s c / 1$ and $\mathrm{M} 41 \Delta \mathrm{sc} / 1$ mutants (Fig. 4B). In addition, we compared biofilm thicknesses formed on CAF-derived ECM by GAS M41 WT and M41 $\Delta$ scl1 mutant strains (Fig. 4C). As expected, the M41 WT GAS formed biofilms that averaged $20 \mu \mathrm{m}$ in 


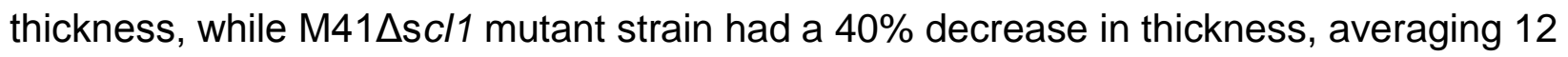
$\mu \mathrm{m}$.

\section{Scl1 mediates GAS colonization of matrix deposited by osteosarcoma cells - In} 1891, Dr. William B. Coley injected inoperable osteosarcomas with live group A streptococci and observed tumor shrinkage and increased survival in some patients (minireview by (58)). While the likely success of Coley's treatment was principally due to the stimulation of immune responses by GAS within tumors $(59,60)$, it is possible that Scl1 indirectly, although relevantly, contributed to treatment success by promoting GAS to colonize the microenvironment of osteosarcomas. Here, we applied the same in vitro model as above, but using Saos-2 osteosarcoma cell line (61). Saos-2 cells were grown to confluency; cells were removed from the matrices that were visualized by Ponceau $S$ staining (Fig. 5A) and characterized for ECM composition by ELISA (Fig. 5B). As before, Ponceau $S$ staining showed the formation fibrillary matrix characteristic of $\mathrm{Fn}$ and $\mathrm{TnC}$ (Fig. 5A), and ELISA data demonstrated that Saos-2 cells deposited some EDAcontaining $\mathrm{cFn}$, as well as considerable amounts of EDB-rich cFn isoforms and $\mathrm{TnC}$ (Fig. $5 B)$, relative to BSA background.

To assess if Scl1 promotes GAS attachment and colonization of Saos-2-deposited matrices, we performed a 1-hour whole-cell attachment assay, using GFP-expressing M1 and M41 isogenic GAS strains (Fig. $5 \mathrm{C}$ ). There were more fluorescent M1 and M41 WT GAS on the Saos-2-deposited matrices than the M1 $\Delta \mathrm{s} c / 1$ mutant and M41 $\Delta \mathrm{s} c / 1$ mutant cells (Fig. 5C, top panels). Quantification revealed significant differences in the attachment between isogenic strain pairs; there was $~ 80 \%$ reduction in the attachment of the $\mathrm{M} 1 \Delta \mathrm{sc} / 1$ mutant and $\sim 90 \%$ reduction by the M41 $1 \mathrm{sc} / 1 \mathrm{GAS}$ (Fig. $5 \mathrm{C}$, bottom graph). In addition to facilitating GAS attachment, Scl1 contributes to biofilm formation on Saos2-deposited matrices (Fig. 5D). Crystal violet staining after 24 hours showed significantly larger bacterial biomass grown by both WT strains on Saos-2 matrices, compared with their respective $\Delta s c / 1$ mutant counterparts (Fig. 5D). In summary, our data demonstrates that Scl1 targets GAS to colonize the complex ECM deposited by cancer-associated fibroblasts and osteosarcoma cells. 


\section{DISCUSSION}

GAS infections typically start within the wounded portal of entry, which is characterized by a microenvironment rich in $\mathrm{cFn}$ isoforms that contain either EDA and/or EDB, as well as TnC. Here we report that Scl1 of GAS binds multiple wound-associated ECM targets that contain FnIII-type repeats and includes EDA and EDB of cFn, as well as FnIII repeats in TnC (Fig. 6). These interactions occurred through the same conserved binding mechanism mediated by the Scl1-V domain and facilitated GAS adherence and biofilm formation.

Group A streptococcal strains may express numerous Fn-binding proteins on the cell surface and at least 11 distinct Fn adhesins have been reported (reviewed in (62)). The most common mechanism of $\mathrm{Fn}$ binding is via the classical Fn-binding repeats which are found in several Fn-binding proteins of GAS, such as protein F1/Sfbl and F2/PFBP, serum opacity factor SOF/Sfbll, FbaA and FbaB, SfbX, and Fbp54 (63-68). During the binding process, the Fn-binding repeats of those adhesins, interact with the $\mathrm{N}$-terminal fibronectin type I repeats $(\mathrm{Fnl})$ through a tandem $\beta$-zipper mechanism and contribute an additional anti-parallel $\beta$-strand within the $\square$-sheet structure of the Fnl repeats $(64,69)$. Scl1, however, lacks the classical Fn-binding repeats and binds, instead, to the Fnlll repeat EDA, via its globular $V$ domain (22). Scl1-EDA binding mechanism was mediated by the C-C' loop of EDA and surface-exposed loops of the Scl1-V domain (19). Here, we determined that several rScl1 variants, with diverse V-domain sequences, bind to FnIIIderived rEDB polypeptide. Using a series of engineered rScl constructs, we furthermore showed that Scl1-EDB binding engaged, like EDA binding, the Scl1-V-domain loops and suggests the same conserved binding mechanism. Homology modeling of EDB and analysis of the predicted loop sequences imply that the C-C' loop of EDB is a potential Scl1-binding target. To our knowledge, Scl1 is the first bacterial adhesin that directly binds the FnllI-EDB of $\mathrm{cFn}$.

In addition, we show that the Scl1-EDB interaction facilitates GAS biofilm formation in vitro on rEDB-coated surfaces. The role of EDB in bacterial infections is beginning to be appreciated. Recently, cFns that include the EDB segment were shown to be upregulated in the cerebral spinal fluid and serum of patients suffering from Staphylococcus aureus meningitis (70). EDB-containing cFns in these patients were 
released by immune cells during phagocytosis of $S$. aureus and enhanced bacterial removal by phagocytic cells. Phagocytosis of $S$. aureus in vitro was augmented by the addition of exogenous $\mathrm{EDB} / \mathrm{cFn}$, which activated the $\alpha_{\mathrm{v}} \beta_{3}$ integrin on phagocytic cells. However, it was not shown if $S$. aureus adhered directly to EDB (70). Similarly, EDA/cFn isoforms were shown to be elevated in a post-surgical bacteremia model in sheep; however, the role of EDA/cFn in facilitating phagocytosis was not investigated (71).

Other bacterial adhesins may also bind to EDA and/or EDB, like Tp0155 of Treponema pallidum and YadA of Yersinia spp. (72). The outer envelope protein Tp0155 of $T$. pallidum displayed a selective binding to $\mathrm{cFn}$ (referred to in text as insoluble matrix fibronectin), but not plasma pFn, which contributed to T. pallidum host-cell adhesion (73). YadA of $Y$. pseudotuberculosis and $Y$. enterocolitica also demonstrated preferential binding to $\mathrm{cFn}$, promoting bacterial cell entry $(74,75)$. Further investigations are needed to assess $\mathrm{EDA} / \mathrm{cFn}$ and/or EDB/cFn binding by these adhesins.

Two streptococcal proteins have been reported to bind to the Fnlll repeats of $\mathrm{TnC}$ (TnFnIII): protein $\mathrm{H}$ (76) and protein F2 (77). The M-like protein $\mathrm{H}$ binds factor $\mathrm{H}$ and IgG, as well as the neural cell adhesion molecule (N-CAM), which contains Fnlll repeats (78). Protein $\mathrm{H}$ binding to $\mathrm{N}$-CAM was competitively inhibited with $\mathrm{pFn}$ fragments encompassing the FnIII repeat region, as well as with two different Fnlll-containing recombinant proteins derived from $\mathrm{TnC}$ (76). Protein F2 contains the prototypical fibronectin-binding repeats, that bind to the N-terminal type I and II repeats of Fn $(66,79)$. To our knowledge, direct binding to the FnIII repeats in either fibronectin or TnC by F2 protein has not been reported. A recent study showed that a protein F2-deficient mutant of an M3-GAS strain bound significantly less TnC compared with the wild-type parental organism, which was restored by in-trans complementation (77). It should be noted that the M3 strain used in this study, MGAS315, does not express Scl1 adhesin (80). Here we used defined recombinant TnFnlll fragments, rTnFn1-5 and rTnFn6-8, and show for the first time that Scl1 binds directly to TnFnlll repeats of $\mathrm{TnC}$.

Cellular fibronectin isoforms and tenascin-C are also present in the tumor microenvironment. In cancerous tissues, cancer cells activate the surrounding stromal fibroblasts, known as cancer-associated fibroblasts (CAFs) $(38,81,82)$. Here, we show that cultured CAFs, isolated from a stage IV laryngeal primary tumor, deposit cFn 
isoforms that contains EDA and/or EDB, as well as TnC in vitro; and that Scl1 facilitates GAS adherence to and biofilm formation on CAF-deposited ECM preparations. GAS has a known connection to cancer, as an original and major component of Coley's toxin (succinctly reviewed in (58)). Developed by Dr. William Coley, a bone sarcoma surgeon (1862-1936), Coley's toxin was the first use of a cancer immunotherapy in medicine $(58,83)$. Coley and colleagues injected live and killed streptococci to treat patients afflicted with soft-tissue and bone tumors with relative success (84). Interestingly, we now know that osteosarcoma cells upregulate $\mathrm{cFn}$ isoforms that contain EDA and/or EDB, and TnC expression (85-87). Here we used model osteosarcoma Saos-2 cells to produce matrices containing the cFn isoforms that contain EDA and/or EDB, as well as TnC. Scl1 promoted GAS colonization and biofilm formation on osteosarcoma-derived matrices. This work adds a new dimension to the history of Coley's toxin, through the capacity of the Scl1 adhesin to target ECM proteins that are present within the tumor microenvironment; this provides a previously unrealized feature in the utilization of GAS in the potential treatment of cancer $(59,60)$.

Understanding of the GAS-fibronectin interaction has evolved since our finding that the GAS adhesin, Scl1, selectively binds to $c F n$, but not $\mathrm{pFn}$, (21) via unique mechanism involving the cFn type III repeat, EDA $(19,22)$. Here, we demonstrate that Scl1 also binds to EDB in isoforms of cFn, as well as to the Fnlll repeats within TnC. All three ECM ligands are expressed within wounded tissue, which is typically the first site GAS encounters within the host. In each case, surface-exposed loops of the globular sequence-variable but structurally-conserved V-domain of Scl1 were responsible for ligand recognition and binding. The Scl1 adhesin improves GAS adherence and biofilm formation on extracellular matrices rich in cFn isoforms that contain EDA and/or EDB, and TnC. Interestingly, the aforementioned ECM components are also constituents of tumor microenvironments and our initial experiments suggest Scl1 has the capacity for targeting tumors. In summary, the Scl1-V domain has evolved within the wound microenvironment, driven by selection for binding to multiple ECM components containing Fnlll repeats. 


\section{EXPERIMENTAL PROCEDURES}

Bacterial strains and growth - Group A Streptococcus (GAS) strains MGAS5005-M1 and MGAS6183-M41, as well as their scl1-inactivated isogenic mutants were used in this study (88,89);Lukomski, $2000 \# 119973 ; H a n, 2006 \# 119967\}$. Briefly, both mutants were generated by allelic replacement with nonpolar resistance cassettes, encoding spectinomycin (MGAS5005 $\Delta s c / 1$ ) and erythromycin (MGAS6183 $\Delta s c / 1)$ resistance. GAS cultures were grown at $37^{\circ} \mathrm{C}$, with $5 \% \mathrm{CO}_{2}$ in Todd-Hewitt broth supplemented with $0.2 \%$ yeast extract, and on Brain Heart Infusion (BHI) agar. For GAS antibiotic selection, erythromycin $\left(4 \mathrm{~g} \mathrm{~m} \mathrm{~m}^{-1}\right)$, chloramphenicol $\left(5-10 \mu \mathrm{g} \mathrm{ml}^{-1}\right)$, and spectinomycin $\left(100 \mu \mathrm{g} \mathrm{ml}^{-}\right.$ $\left.{ }^{1}\right)$ were added to the medium.

Complementation of M1 group A Streptococcus - To complement MGAS5005 Ascl1 with either scl1.1 or scl.chi2 in-trans, the E.coli shuttle vector pSB207 was used (90). Briefly, a DNA fragment, encompassing the scl1.1 coding sequence with upstream promoter, was PCR-amplified from genomic DNA and cloned into pSB207, generating plasmid pSL620 (19). A synthetic double-stranded DNA fragment (gBlocks; Integrated DNA Technologies) encoding the scl.chi2 coding sequence was cloned into pSB620, generating pSL621 (19). Clones were verified by sequencing and were then introduced into MGAS5005 $\Delta s c / 1$ mutant; transformants were selected on BHI agar containing $10 \mu \mathrm{g}$ $\mathrm{ml}^{-1}$ chloramphenicol and GAS cultures were routinely grown in THY broth with $10 \mu \mathrm{g} \mathrm{ml}^{-}$ ${ }^{1}$ chloramphenicol.

Recombinant protein production - Production of recombinant streptococcal collagenlike proteins ( $\mathrm{rScls}$ ). Gene cloning and $\mathrm{rScl}$-protein production were performed in Escherichia coli DH5a, TB1 and BL-21 strains grown in Luria-Bertani (LB) media with ampicillin $\left(100 \mathrm{mg} \mathrm{ml}^{-1}\right)$ at $37^{\circ} \mathrm{C}$. rScl-encoding clones, which were derived from the original scl alleles, were generated by PCR amplification from GAS genomic DNA and cloned into the E.coli expression vector pASK-IBA2(15) (16). Clones encoding the chimeric $\mathrm{rScl}$ proteins were generated using synthetic double-stranded DNA fragments (gBlocks; Integrated DNA Technologies), as described previously (19). All plasmids were verified by DNA sequencing. 
All rScl proteins were generated using the Strep-tag II cloning, expression, and purification system (IBA-GmbH). Proteins were expressed with a C-terminal affinity tag and purified on Strep-Tactin sepharose, as described $(15,16)$. The rScl1.1 protein is derived from Scl1 protein in M1-type strain MGAS6708 (15); rScl1.28 originates from M28 strain MGAS6274 (15), rScl1.41 is derived from M41 strain MGAS6183 (91), rScl1.3 is derived from M3 strain MGAS315 (80), rScl1.12 is derived from an M12 strain MGAS6139 (16), rScl1.2 is derived from an M2 strain MGAS3803 (92), and rScl2.4 is derived from M4 strain MGAS321 (16). Both naturally-derived and chimeric $\mathrm{rScl}$ proteins were expressed in E. coli BL21 periplasm following induction with anhydrotetracycline at 0.2 $\mu \mathrm{g} \mathrm{mL}^{-1}$ for 3 hours. Cells were centrifuged and suspended either in high sucrose buffer (100mM Tris-HCl, 1 mM EDTA, pH 8.0, 500mM sucrose) or Cell Lytic B Buffer (Sigma), for separation of the periplasmic fraction and subsequent affinity purification. Purified proteins were analyzed by SDS-PAGE and stained with RAPIDstain ${ }^{\mathrm{TM}}$; proteins were dialyzed against $25 \mathrm{mM} \mathrm{HEPES}, \mathrm{pH} 8.0$, and stored at $-20^{\circ} \mathrm{C}$. Protein concentrations were determined using Qubit fluorometric quantitation.

Recombinant extra domain $B$ ( $r E D B$ ) production - $r E D B$ was produced using the $p Q E-$ 30 His-tag cloning, expression, and purification system in the E. coli strain JM-109, as described elsewhere (93). EDB-encoding segment was amplified by PCR from rat cDNA and cloned into PQE-30; the resulting construct was a gift from Dr. John Peters. Protein expression was induced with $1 \mathrm{mM}$ isopropyl $\beta$-D-1-thiogalactopyranoside for 3 hours. Cells were harvested by centrifugation, and pellets were frozen at $-20^{\circ} \mathrm{C}$ for 2 hours or overnight. Cells were next suspended in a bacterial lysis buffer $(50 \mathrm{mM}$ Tris/HCL pH 8.0, $50 \mathrm{mM} \mathrm{NaCl}, 2 \mathrm{mM} \mathrm{MgCl}$, 2\% Triton X-100, $10 \mathrm{mM} \beta$-mercaptoethanol, $0.2 \mathrm{mg} / \mathrm{ml}$ lysozyme, 1 EDTA-free protease inhibitor cocktail tablet [per $10 \mathrm{~mL}$ ], $1 \mathrm{mM}$ phenylmethane sulfonyl fluoride, $10 \mathrm{U} \mathrm{ml} \mathrm{DNasel)} \mathrm{and} \mathrm{incubated} \mathrm{on} \mathrm{ice} \mathrm{for} 20$ minutes. Cell lysate was centrifuged at high speed (16000g $\times 20$ minutes) and supernatant was collected. Supernatant was mixed 1:1 volumetrically with wash buffer $\left(50 \mathrm{mM} \mathrm{NaH}_{2} \mathrm{PO}_{4}\right.$, $10 \mathrm{mM}$ imidazole, $300 \mathrm{mM} \mathrm{NaCl}$ ), added to $1 \mathrm{ml}$ of cobalt-agarose resin, and then poured into a column. Sample was washed 10x resin bed volume with wash buffer, and then rEDB protein was eluted in elution buffer (50 mM NaH $2 \mathrm{PO}_{4}, 150 \mathrm{mM}$ imidazole, $300 \mathrm{mM}$ 
$\mathrm{NaCl}$ ). Purified protein was dialyzed against $25 \mathrm{mM} \mathrm{HEPES}$ buffer, $\mathrm{pH} 8.0$ and stored at $-20^{\circ} \mathrm{C}$ until future use. Protein integrity and purity were assessed by $18 \%$ SDS-PAGE and concentration was measured with Qubit fluorometric quantitation.

Recombinant tenascin-C fibronectin type III fragments (rTnFnIII) - Recombinant tenascin-C fibronectin type III (FnIII) fragments: repeats 1-5, (rTnFn1-5), repeats 6-8, (rTnFn6-8), or repeat 3 ( $r T n F n 3)$ were produced after recloning into pQE-30 His-tag vector in the E. coli strain XL1-blue. Sequences for each fragment were PCR-amplified from the original constructs cloned in pET15b ( $r T n F n 1-5$ and $r T n F n 6-8)$ or pET11b (rTnFn3) expression vectors (provided by Dr. Harold P. Erickson)(94). Recombinant proteins were expressed, purified, and stored as described above with rEDB.

Homology modeling of, EDA, EDB, and TnFnIII domains - The homology models of tenascin-C domains from 1 to 5 (TnFn1-5) and from 6 to 8 (TnFn6-8) were obtained after consensus-based sequence alignment using the profile hidden Markov models (profile HMMs) implemented in the program HHpred (95). For TnFn1-5, best template model was identified as the structure of the recombinant four-domain fragment FnIII 7B89 (PDB code $3 t 1 \mathrm{w}$, seqid $27.7 \%$ with repeats $1-3$ and $25.2 \%$ with repeats $2-5$ ). In the case of TnFn6-8, best template was the structure of human fibronectin domains 12-14 (PDB code 3r8q, seqid 34.9\%). Once the best templates were identified, homology models were built with MODELLER (95). Stereo chemical quality of predicted models was improved by energy minimization using GROMACS (96). Validation of the structural quality of predicted models was carried out using the PDBsum database at PROCHECK server (http://www.ebi.ac.uk/thornton-srv/software/PROCHECK/).

Protein binding assays $-\mathrm{rScl}$ proteins $(0.5 \mu \mathrm{M}$ solutions) were immobilized onto StrepTactin-coated microplate wells for $1.5 \mathrm{~h}$ at room temperature and blocked with $1 \times$ TBS (25 $\mathrm{mM}$ Tris, $150 \mathrm{mM}$ sodium chloride, $\mathrm{pH}$ 7.4) supplemented with $1 \%$ fetal bovine serum albumin overnight at $4^{\circ} \mathrm{C}$, followed by incubation with ECM ligands: rEDB, tenascin-C (TnC) purified from glioblastoma cells (Sigma), rTnFn1-5, rTnFn6-8, and rTnFn3. The no rScl controls were performed in BSA-coated wells for each ligand and each antibody 
used. Final OD values were normalized by subtracting the BSA controls in each experimental set-up. ECM ligands were added to the rScl-immobilized wells at $1 \mu \mathrm{g}$ per well (except for $\mathrm{TnC}$, incubated at $0.5 \mu \mathrm{g}$ per well) and incubated at room temperature for 1 hour. Bound rECM ligands were detected with monoclonal antibodies (mAbs): anti-Histag for rEDA and rEDB (Proteintech; 1:1000), anti-TnC BC-24, specific for the epidermal growth factor-like repeats, (ThermoFisher; 1:1000), followed by goat anti-mouse secondary antibody conjugated to horseradish peroxidase (HRP) (Jackson ImmunoResearch; 1:1000). The HRP substrate, 2,20-azino-bis(3-ethylbenzthiazoline-6sulphonic acid) (ThermoFisher; ABTS) was used and colorimetric reactions were recorded at $\mathrm{OD}_{415} \mathrm{~nm}$.

\section{Eukaryotic cell assays}

Isolation of cancer-associated fibroblasts (CAFs) - CAFs were isolated from a stage IV laryngeal cancer resection obtained from the West Virginia University Pathology Laboratory for Translational Medicine in compliance with approved Institutional Review Board protocol \#1310105737A033 as described previously (97). Briefly, tissue was submerged in DMEM/10\% FBS containing $1 \%$ penicillin-streptomycin-amphotericin B (P$\mathrm{S}-\mathrm{A}$ ) (Millipore, 51610420ML) and mechanically digested. Tissue pieces $<2 \mathrm{~mm}$ were placed into a 24-well plate, allowed to adhere for 2-3 min, then covered with media and placed in a humidified $37^{\circ} \mathrm{C}$ incubator with $5 \% \mathrm{CO}_{2}$. Emanating $\mathrm{CAFs}$ were cultured in DMEM supplemented with 10\% FBS/1\% P-S-A, passaged 2-3 times with Accumax (Millipore, SCR006) until acclimated, then passaged with $0.25 \%$ trypsin and grown in DMEM/10\% FBS. CAFs were passaged 5 times before being cryopreserved. Thawed CAFs were passaged 2 times prior to use.

Preparation of cancer-cell-derived extracellular matrices - Cancer-associated fibroblasts (CAFs) and the osteosarcoma cell line Saos-2 (ATCC $®$ HTB-85) were used to produce cell-derived extracellular matrices. Matrices were prepared, as described previously (22), Briefly, CAFs and Saos-2 cells were cultured in high-glucose Dulbecco's Modified Eagle Medium with 10\% fetal bovine serum and 1\% penicillin and streptomycin 
at $37^{\circ} \mathrm{C}$ in an atmosphere of $5 \% \mathrm{CO}_{2}$ throughout the experiment. To produce the extracellular matrix for specific tests, cells were grown as follows: (i) for matrix characterization via immunofluorescence or for GFP expressing GAS (GFP-GAS) attachment assays, cells were grown on $15-\mathrm{mm}$ glass coverslips inserted into wells of (24-well) tissue culture plates, and (ii) for crystal violet biofilm assay, matrix characterization by ELISA or by Ponceau $S$ staining, cells were growth in plastic wells without glass coverslips. Cells were seeded at 50,000 cells per well, grown until confluent, and then removed through treatment with $5 \mathrm{mM}$ ethylene glycol tetraacetic acid (EGTA). Samples were washed gently with PBS and wells or coverslips were subsequently used for assessment.

\section{Cell-derived ECM matrix characterization}

Ponceau S staining - To visualize the matrices deposited by CAFs and Saos-2 cells, Ponceau $S$ solution $(0.1 \%$ in $5 \%$ acetic acid solution) was added to the wells for 20 minutes. Stain was then removed, and wells were examined microscopically using a Zeiss Axiovert $40 \mathrm{CFL}$ microscope with a 20x objective. Image acquisition was done using the Zeiss AxioCam Mrc5 camera and images analyzed with Zeiss AxioVision 4.8 software.

Matrix characterization by ELISA - CAFs- and Saos-2-derived matrices were prepared, as above, and wells were blocked overnight with $1 \%$ bovine serum albumin in $1 \mathrm{X}$ TBS. The next day, the following mAbs were added to wells for 1 hour at room temperature: anti-fibronectin, specific for the fourth fibronectin type III repeat of fibronectin, (Sigma; IST4, 1:1000), anti-EDA (Santa Cruz Biotechnology; IST9, 1:1000), anti-EDB containing cFn (Sirius Biotechnology; C6, 1:500), or BC-24, (1:1000); a goat anti-mouse secondary antibody conjugated with HRP (1:2000) was added for 1 hour, washed, and then developed with ABTS substrate. Each antibody was used in triplicate wells, over 3 different experiments. Wells with secondary antibody only were used for background correction. 
Matrix characterization by immunofluorescence microscopy - To visualize the matrices deposited on glass coverslips, wells were denuded of cells and blocked overnight at $4^{\circ} \mathrm{C}$ with $1 \% \mathrm{BSA} / \mathrm{TBS}$. Anti-ECM antibodies: IST4 (1:100), IST9 (1:200), C6 (1:100) and BC-24 (1:1000) were added to wells for 1 hour at room temperature, washed with TBS, followed by addition of goat anti-mouse secondary Ab conjugated with Alexa Fluor 568® (Thermofisher, 1:300). Coverslips were then washed and mounted in ProLong Gold (Invitrogen). Matrices were visualized using a Nikon A1R confocal microscope equipped with a $60 x$ objective. Images were processed using Nikon NIS-Elements Software.

\section{GAS attachment and biofilm assays}

Scl1 surface expression and rEDB binding by flow cytometry - Determination of Sclsurface expression by GAS cells, as well as rEDB binding to whole-GAS cells were measured by flow cytometry. Bacteria were grown to an $\mathrm{OD}_{600}$ of 0.5 , harvested by centrifugation and washed with flow cytometry buffer (phosphate-buffered saline containing $10 \%$ Todd-Hewitt broth with $0.2 \%$ yeast extract). For Scl-surface detection, the anti-Scl1.1-V antibody (11), pre-absorbed with MGAS5005 $\Delta s c / 1$ cells, was incubated with GAS cells for 30 minutes on ice. Cells were next washed and incubated with Allophycocyanin (APC)-conjugated donkey anti-rabbit antibody (Jackson ImmunoResearch; 1:150). For ligand binding, GAS cells tested were incubated with rEDB for 30 minutes at room temperature. GAS cells were then centrifuged and washed $2 x$ with flow cytometry buffer. Bound protein was detected with anti-His-tag mAb (1:750), followed by a goat anti-mouse secondary pAb conjugated with Alexa Fluorß 568 (1:150). Cells were washed, fixed in $0.4 \%$ paraformaldehyde and analyzed. 50,000 events were collected per sample using a BD LSRFortessa flow cytometer and data were analyzed with FCS Express Flow 6 software.

GAS whole-cell attachment assay - GFP-GAS adherence was studied on glass coverslips with rEDB coating and on cancer-cell-derived matrices, as described (21). 1 $\mathrm{ml}$ of GFP-GAS cultures, prepared as above, were seeded into wells and incubated for 1 
hour at $37^{\circ} \mathrm{C}$, washed with PBS, fixed with $3 \%$ paraformaldehyde for 30 minutes, and then mounted in ProLong Gold overnight. The total number of GAS cells were counted in 10 arbitrary fields and the numbers of WT M1 or M41 GAS cells were set as $100 \%$. The differences between WT and isogenic $\square s c / 1$ mutants were evaluated statistically.

Crystal violet staining of GAS biofilms - GAS biofilm formation was tested on rEDBcoated surfaces and on cancer-cell-derived matrices. Wild-type and isogenic scl1 mutant GAS strains were grown to an $\mathrm{OD}_{600}$ of 0.5 and 1-ml aliquots were seeded into 24-well culture plates coated either with rEDB $(2 \mu \mathrm{g} /$ well) or in wells containing CAF- or Saos-2derived extracellular matrices, prepared as above. GAS biofilms were grown for 24 hours, washed with PBS, and stained with 1\% (v/v) crystal violet solution (Becton Dickinson) for 30 minutes at room temperature. Biomass staining was solubilized with $0.5 \mathrm{ml}$ of $90 \%$ ethanol and assessed spectrophotometrically at $\mathrm{OD}_{600 \mathrm{~nm}}$.

Confocal laser scanning microscopy of GAS biofilms - GAS biofilms were also visualized on glass coverslips coated with rEDB $(2 \mu \mathrm{g})$ or on cancer-cell-derived matrices, using isogenic GFP-GAS (21,98). 24-hour biofilms were grown as above, fixed in 3\% paraformaldehyde for 30 minutes, and mounted in ProLong Gold overnight. Biofilms were imaged using a Nikon A1R confocal microscope, with a 100x objective. Images were analyzed and deconvoluted using NIS-Elements Software. Conversion to threedimensional images was performed with conventional Z-stacks, deconvoluted stepwise and transformed using NIS-Elements Software.

Statistical analyses - Statistics were performed using the two-tailed paired Student's $t$ test. Significance was denoted at levels of ${ }^{*} P \leq 0.05,{ }^{* *} P \leq 0.01$, or ${ }^{* * *} P \leq 0.001$. Error bars represent standard deviations with analyses based on three independent experimental repeats $(\mathrm{N}=3)$, each performed in triplicate technical replicates.

\section{ACKNOWLEDGEMENTS}

We thank Paolo Fagone for assistance with rEDB purification, Kathleen Brundage for flow cytometry assistance, and Amanda Ammer and Karen Martin for microscopy assistance. 
Thanks also go to John Peters for providing rEDA and rEDB expression plasmids, and to Harold Erickson for TnFnlll encoding plasmids. We also thank Nyles Charon and Beth Bachert for critical review of the manuscript. Flow cytometry experiments were performed in the West Virginia University Flow Cytometry \& Single Cell Core Facility, which is supported by the General Medicine Sciences of the National Institute of General Medicine of the National Institutes of Health under grant numbers: P30GM103488 (CoBRE) and 1P20GM121322-01A1 (CoBRE). The BD Fortessa is supported by a grant from the National Institute of General Medicine of the National Institutes of Health under grant number ODO16165. Imaging was done through the West Virginia University Imaging Facility, which is supported in part by the National Institute of General Medical Sciences of the National Institutes of Health, under grant numbers: P20GM103434 and U45GM104942. This work was supported in part by the National Institutes of Health Grants AI50666 and by a West Virginia University HSC Bridge Grant Funding from the Office of Research and Graduate Education (SL); DHM was supported by the Integrative Graduate Education and Research Training (IGERT) Traineeship for Research and Education in Nanotoxicology under award number 1144676 and by the Dr. Jennifer Gossling Scholarship in Microbiology. The authors report no conflicts of interest with the contents of this article. The content is solely the responsibility of the authors and does not necessarily represent the official views of the National Institutes of Health.

\section{REFERENCES}

1. Carapetis, J. R., Steer, A. C., Mulholland, E. K., and Weber, M. (2005) The global burden of group A streptococcal diseases. Lancet Infect. Dis. 5, 685-694

2. Bisno, A. L., Pearce, I. A., Wall, H. P., Moody, M. D., and Stollerman, G. H. (1970) Contrasting epidemiology of acute rheumatic fever and acute glomerulonephritis. N. Engl. J. Med. 283, 561565

3. Swedo, S. E., Leonard, H. L., Mittleman, B. B., Allen, A. J., Rapoport, J. L., Dow, S. P., Kanter, M. E., Chapman, F., and Zabriskie, J. (1997) Identification of children with pediatric autoimmune neuropsychiatric disorders associated with streptococcal infections by a marker associated with rheumatic fever. Am. J. Psychiatry 154, 110-112

4. Cunningham, M. W. (2000) Pathogenesis of group A streptococcal infections. Clin. Microbiol. Rev. 13, 470-511 
5. Ralph, A. P., and Carapetis, J. R. (2013) Group a streptococcal diseases and their global burden. Curr. Top. Microbiol. Immunol. 368, 1-27

6. Efstratiou, A., and Lamagni, T. (2016) Epidemiology of Streptococcus pyogenes. In Streptococcus pyogenes: Basic biology to clinical manifestations [Internet] (Ferretti, J. J., Stevens, D. L., and Fischetti, V. A. eds.), University of Oklahoma Health Sciences Center, Oklahoma City, OK.

7. Fischetti, V. (2016) M Protein and other surface proteins on streptococci. In Streptococcus pyogenes : Basic biology to clinical manifestations [Internet] (Ferretti JJ, S. D., Fischetti VA ed.), Univeristy of Oklahoma Health Sciences Center, Oklahoma City, OK.

8. Sanderson-Smith, M., De Oliveira, D. M., Guglielmini, J., McMillan, D. J., Vu, T., Holien, J. K., Henningham, A., Steer, A. C., Bessen, D. E., Dale, J. B., Curtis, N., Beall, B. W., Walker, M. J., Parker, M. W., Carapetis, J. R., Van Melderen, L., Sriprakash, K. S., and Smeesters, P. R. (2014) A systematic and functional classification of Streptococcus pyogenes that serves as a new tool for molecular typing and vaccine development. J. Infect. Dis. 15; 1325-38

9. Walker, M. J., Barnett, T. C., McArthur, J. D., Cole, J. N., Gillen, C. M., Henningham, A., Sriprakash, K. S., Sanderson-Smith, M. L., and Nizet, V. (2014) Disease manifestations and pathogenic mechanisms of group A Streptococcus. Clin. Microbiol. Rev. 27, 264-301

10. Rasmussen, M., Edén, A., and Björck, L. (2000) SclA, a novel collagen-like surface protein of Streptococcus pyogenes. Infect. Immun. 68, 6370-6377

11. Lukomski, S., Nakashima, K., Abdi, I., Cipriano, V. J., Ireland, R. M., Reid, S. D., Adams, G. G., and Musser, J. M. (2000) Identification and characterization of the $s c /$ gene encoding a group $A$ Streptococcus extracellular protein virulence factor with similarity to human collagen. Infect. Immun. 68, 6542-6553

12. Lukomski, S., Nakashima, K., Abdi, I., Cipriano, V. J., Shelvin, B. J., Graviss, E. A., and Musser, J. M. (2001) Identification and characterization of a second extracellular collagen-like protein made by group A Streptococcus: control of production at the level of translation. Infect. Immun. 69, 1729-1738

13. Rasmussen, M., and Björck, L. (2001) Unique regulation of SclB - a novel collagen-like surface protein of Streptococcus pyogenes. Mol. Microbiol. 40, 1427-1438

14. Whatmore, A. M. (2001) Streptococcus pyogenes sclB encodes a putative hypervariable surface protein with a collagen-like repetitive structure. Microbiology 147, 419-429

15. Xu, Y., Keene, D. R., Bujnicki, J. M., Höök, M., and Lukomski, S. (2002) Streptococcal Scl1 and Scl2 proteins form collagen-like triple helices. J Biol Chem 277, 27312-27318

16. Han, R., Zwiefka, A., Caswell, C. C., Xu, Y., Keene, D. R., Lukomska, E., Zhao, Z., Höök, M., and Lukomski, S. (2006) Assessment of prokaryotic collagen-like sequences derived from streptococcal Scl1 and Scl2 proteins as a source of recombinant GXY polymers. Appl. Microbiol. Biotechnol. 72, 109-115 
17. Squeglia, F., Bachert, B., Romano, M., Lukomski, S., and Berisio, R. (2013) Crystallization and preliminary X-ray crystallographic analysis of the variable domain of Scl2.3, a streptococcal collagen-like protein from invasive M3-type Streptococcus pyogenes. Acta Crystallogr. Sect. F Struct. Biol. Cryst. Commun. 69, 1023-1025

18. Squeglia, F., Bachert, B., De Simone, A., Lukomski, S., and Berisio, R. (2014) The crystal structure of the streptococcal collagen-like protein 2 globular domain from invasive M3-type group A Streptococcus shows significant similarity to immunomodulatory HIV protein gp41. J Biol Chem 289, 5122-5133

19. McNitt, D. H., Choi, S. J., Keene, D. R., Van De Water, L., Squeglia, F., Berisio, R., and Lukomski, S. (2018) Surface-exposed loops and an acidic patch in the Scl1 protein of group A Streptococcus enable Scl1 binding to wound-associated fibronectin. J. Biol. Chem. 293, 77967810

20. Lukomski, S., Bachert, B. A., Squeglia, F., and Berisio, R. (2017) Collagen-like proteins of pathogenic streptococci. Mol. Microbiol. 103, 919-930

21. Caswell, C. C., Oliver-Kozup, H., Han, R., Lukomska, E., and Lukomski, S. (2010) Scl1, the multifunctional adhesin of group A Streptococcus, selectively binds cellular fibronectin and laminin, and mediates pathogen internalization by human cells. FEMS Microbiol. Lett. 303, 61-68

22. Oliver-Kozup, H., Martin, K. H., Schwegler-Berry, D., Green, B. J., Betts, C., Shinde, A. V., Van De Water, L., and Lukomski, S. (2013) The group A streptococcal collagen-like protein-1, Scl1, mediates biofilm formation by targeting the extra domain A-containing variant of cellular fibronectin expressed in wounded tissue. Mol. Microbiol. 87, 672-689

23. Ffrench-Constant, C. (1995) Alternative splicing of fibronectin--many different proteins but few different functions. Exp. Cell Res. 221, 261-271

24. Hynes, R. O. (1990) Fibronectins, Springer-Verlag, New York, NY

25. Pankov, R., and Yamada, K. M. (2002) Fibronectin at a glance. J. Cell Sci. 115, 3861-3863

26. To, W. S., and Midwood, K. S. (2011) Plasma and cellular fibronectin: distinct and independent functions during tissue repair. Fibrogenesis Tissue Repair 4, 21

27. Leahy, D. J., Aukhil, I., and Erickson, H. P. (1996) 2.0 Å crystal structure of a four-domain segment of human fibronectin encompassing the RGD loop and synergy region. Cell 84, 155-164

28. Niimi, T., Osawa, M., Yamaji, N., Yasunaga, K., Sakashita, H., Mase, T., Tanaka, A., and Fujita, S. (2001) NMR structure of human fibronectin EDA. J. Biomol. NMR 21, 281-284

29. Ffrench-Constant, C., and Hynes, R. O. (1989) Alternative splicing of fibronectin is temporally and spatially regulated in the chicken embryo. Development 106, 375-388

30. Oyama, F., Murata, Y., Suganuma, N., Kimura, T., Titani, K., and Sekiguchi, K. (1989) Patterns of alternative splicing of fibronectin pre-mRNA in human adult and fetal tissues. Biochemistry 28 , 1428-1434 
31. Ffrench-Constant, C., Van De Water, L., Dvorak, H. F., and Hynes, R. O. (1989) Reappearance of an embryonic pattern of fibronectin splicing during wound healing in the adult rat. J. Cell Biol. 109, 903-914

32. Zardi, L., Carnemolla, B., Siri, A., Petersen, T. E., Paolella, G., Sebastio, G., and Baralle, F. E. (1987) Transformed human cells produce a new fibronectin isoform by preferential alternative splicing of a previously unobserved exon. EMBO J. 6, 2337-2342

33. Singh, P., Reimer, C. L., Peters, J. H., Stepp, M. A., Hynes, R. O., and Van De Water, L. (2004) The spatial and temporal expression patterns of integrin $\alpha 9 \beta 1$ and one of its ligands, the EIIIA segment of fibronectin, in cutaneous wound healing. J. Invest. Dermatol. 123, 1176-1181

34. Muro, A. F., Chauhan, A. K., Gajovic, S., laconcig, A., Porro, F., Stanta, G., and Baralle, F. E. (2003) Regulated splicing of the fibronectin EDA exon is essential for proper skin wound healing and normal lifespan. J. Cell Biol. 162, 149-160

35. Longmate, W. M., Lyons, S. P., Chittur, S. V., Pumiglia, K. M., Van De Water, L., and DiPersio, C. M. (2017) Suppression of integrin $\alpha 3 \beta 1$ by $\alpha 9 \beta 1$ in the epidermis controls the paracrine resolution of wound angiogenesis. J. Cell Biol. 216, 1473-1488

36. Castellani, P., Viale, G., Dorcaratto, A., Nicolo, G., Kaczmarek, J., Querze, G., and Zardi, L. (1994) The fibronectin isoform containing the ED-B oncofetal domain: A marker of angiogenesis. Int. J. Cancer 59, 612-618

37. Birchler, M. T., Milisavlijevic, D., Pfaltz, M., Neri, D., Odermatt, B., Schmid, S., and Stoeckli, S. J. (2003) Expression of the extra domain B of fibronectin, a marker of angiogenesis, in head and neck tumors. Laryngoscope 113, 1231-1237

38. Gopal, S., Veracini, L., Grall, D., Butori, C., Schaub, S., Audebert, S., Camoin, L., Baudelet, E., Radwanska, A., Beghelli-de la Forest Divonne, S., Violette, S. M., Weinreb, P. H., Rekima, S., Ilie, M., Sudaka, A., Hofman, P., and Van Obberghen-Schilling, E. (2017) Fibronectin-guided migration of carcinoma collectives. Nature Comm. 8, 14105

39. Midwood, K. S., and Orend, G. (2009) The role of tenascin-C in tissue injury and tumorigenesis. $J$ Cell Commun Signal 3, 287-310

40. Karus, M., Denecke, B., Ffrench-Constant, C., Wiese, S., and Faissner, A. (2011) The extracellular matrix molecule tenascin $C$ modulates expression levels and territories of key patterning genes during spinal cord astrocyte specification. Development 138, 5321-5331

41. Sahlberg, C., Aukhil, I., and Thesleff, I. (2001) Tenascin-C in developing mouse teeth: expression of splice variants and stimulation by TGF $\beta$ and FGF. Eur. J. Oral Sci. 109, 114-124

42. Chiquet-Ehrismann, R., Orend, G., Chiquet, M., Tucker, R. P., and Midwood, K. S. (2014) Tenascins in stem cell niches. Matrix Biol. 37, 112-123

43. Chiquet-Ehrismann, R., Mackie, E. J., Pearson, C. A., and Sakakura, T. (1986) Tenascin: an extracellular matrix protein involved in tissue interactions during fetal development and oncogenesis. Cell 47, 131-139 
44. Anthony, B. F. (2000) Streptococcal pyoderma. In Streptococcal infections (Stevens, D. L., and Kaplan, E. L. eds.), Oxford University Press, New York, N. Y. pp 144-151

45. Midwood, K. S., Chiquet, M., Tucker, R. P., and Orend, G. (2016) Tenascin-C at a glance. J. Cell Sci. 129, 4321-4327

46. Midwood, K. S., Valenick, L. V., Hsia, H. C., and Schwarzbauer, J. E. (2004) Coregulation of fibronectin signaling and matrix contraction by tenascin-C and syndecan-4. Mol. Biol. Cell 15, 5670-5677

47. Shinde, A. V., Bystroff, C., Wang, C., Vogelezang, M. G., Vincent, P. A., Hynes, R. O., and Van De Water, L. (2008) Identification of the peptide sequences within the EIIIA (EDA) segment of fibronectin that mediate integrin a9b1-dependent cellular activities. J Biol Chem 283, 2858-2870

48. Yokosaki, Y., Matsuura, N., Higashiyama, S., Murakami, I., Obara, M., Yamakido, M., Shigeto, N., Chen, J., and Sheppard, D. (1998) Identification of the ligand binding site for the integrin $\alpha 9 \beta 1$ in the third fibronectin type III repeat of tenascin-C. J Biol Chem 273, 11423-11428

49. Humphries, J. D., Byron, A., and Humphries, M. J. (2006) Integrin ligands at a glance. J. Cell Sci. 119, 3901-3903

50. McNitt, D. H., Van De Water, L., Marasco, D., Berisio, R., and Lukomski, S. (2018) Streptococcal collagen-like protein 1 binds wound fibronectin: implications in pathogen targeting. Curr. Med.

Chem. 25, 1-1

51. Dvorak, H. F. (2015) Tumors: wounds that do not heal-redux. Cancer Immunol. Res. 3, 1-11

52. Marsh, T., Pietras, K., and McAllister, S. S. (2013) Fibroblasts as architects of cancer pathogenesis. Biochim. Biophys. Acta 1832, 1070-1078

53. Gaggioli, C., Hooper, S., Hidalgo-Carcedo, C., Grosse, R., Marshall, J. F., Harrington, K., and Sahai, E. (2007) Fibroblast-led collective invasion of carcinoma cells with differing roles for RhoGTPases in leading and following cells. Nat. Cell Biol. 9, 1392-1400

54. O'Connell, J. T., Sugimoto, H., Cooke, V. G., MacDonald, B. A., Mehta, A. I., LeBleu, V. S., Dewar, R., Rocha, R. M., Brentani, R. R., Resnick, M. B., Neilson, E. G., Zeisberg, M., and Kalluri, R. (2011) VEGF-A and Tenascin-C produced by S100A4+ stromal cells are important for metastatic colonization. Proc. Natl. Acad. Sci. U. S. A. 108, 16002-16007

55. Mackie, E. J., Chiquet-Ehrismann, R., Pearson, C. A., Inaguma, Y., Taya, K., Kawarada, Y., and Sakakura, T. (1987) Tenascin is a stromal marker for epithelial malignancy in the mammary gland. Proc. Natl. Acad. Sci. U. S. A. 84, 4621-4625

56. Norton, P. A., and Hynes, R. O. (1987) Alternative splicing of chicken fibronectin in embryos and in normal and transformed cells. Mol. Cell. Biol. 7, 4297-4307

57. Rybak, J. N., Roesli, C., Kaspar, M., Villa, A., and Neri, D. (2007) The extra-domain A of fibronectin is a vascular marker of solid tumors and metastases. Cancer Res. 67, 10948-10957

58. McCarthy, E. F. (2006) The toxins of William B. Coley and the treatment of bone and soft-tissue sarcomas. lowa Orthop. J. 26, 154-158 
59. Linnebacher, M., Maletzki, C., Emmrich, J., and Kreikemeyer, B. (2008) Lysates of S. pyogenes serotype M49 induce pancreatic tumor growth delay by specific and unspecific antitumor immune responses. J. Immunother. 31, 704-713

60. Maletzki, C., Linnebacher, M., Kreikemeyer, B., and Emmrich, J. (2008) Pancreatic cancer regression by intratumoural injection of live Streptococcus pyogenes in a syngeneic mouse model. Gut 57, 483-491

61. Fogh, J., Fogh, J. M., and Orfeo, T. (1977) One hundred and twenty-seven cultured human tumor cell lines producing tumors in nude mice. J. Natl. Cancer Inst. 59, 221-226

62. Yamaguchi, M., Terao, Y., and Kawabata, S. (2013) Pleiotropic virulence factor - Streptococcus pyogenes fibronectin-binding proteins. Cell. Microbiol. 15, 503-511

63. Signäs, C., Raucci, G., Jönsson, K., Lindgren, P. E., Anantharamaiah, G. M., Höök, M., and Lindberg, M. (1989) Nucleotide sequence of the gene for a fibronectin-binding protein from Staphylococcus aureus: use of this peptide sequence in the synthesis of biologically active peptides. Proc. Natl. Acad. Sci. U. S. A. 86, 699-703

64. Schwarz-Linek, U., Werner, J. M., Pickford, A. R., Gurusiddappa, S., Kim, J. H., Pilka, E. S., Briggs, J. A., Gough, T. S., Höök, M., Campbell, I. D., and Potts, J. R. (2003) Pathogenic bacteria attach to human fibronectin through a tandem beta-zipper. Nature 423, 177-181

65. Talay, S. R., Valentin-Weigand, P., Jerlstrom, P. G., Timmis, K. N., and Chhatwal, G. S. (1992) Fibronectin-binding protein of Streptococcus pyogenes: sequence of the binding domain involved in adherence of streptococci to epithelial cells. Infect. Immun. 60, 3837-3844

66. Jaffe, J., Natanson-Yaron, S., Caparon, M. G., and Hanski, E. (1996) Protein F2, a novel fibronectin-binding protein from Streptococcus pyogenes, possesses two domains. Mol. Microbiol. 21, 373-384

67. Rakonjac, J. V., Robbins, J. C., and Fischetti, V. A. (1995) DNA sequence of the serum opacity factor of group A streptococci: identification of a fibronectin-binding repeat domain. Infect. Immun. 63, 622-631

68. Terao, Y., Kawabata, S., Kunitomo, E., Murakami, J., Nakagawa, I., and Hamada, S. (2001) Fba, a novel fibronectin-binding protein from Streptococcus pyogenes, promotes bacterial entry into epithelial cells, and the fba gene is positively transcribed under the Mga regulator. Mol. Microbiol. 42, 75-86

69. House-Pompeo, K., Xu, Y., Joh, D., Speziale, P., and Höök, M. (1996) Conformational changes in the fibronectin binding MSCRAMMs are induced by ligand binding. J Biol Chem 271, 1379-1384

70. Kraft, S., Klemis, V., Sens, C., Lenhard, T., Jacobi, C., Samstag, Y., Wabnitz, G., Kirschfink, M., Wallich, R., Hänsch, G. M., and Nakchbandi, I. A. (2016) Identification and characterization of a unique role for EDB fibronectin in phagocytosis. J. Mol. Med. 94, 567-581 
71. Rizk, T. A., Rebres, R. A., Vincent, P. A., Charash, W. E., McKeown-Longo, P. J., Lewis, E. P., Brien, T. P., Minnear, F. L., Fortune, J. B., and Saba, T. M. (1994) Delayed elevation of ED1cellular fibronectin in plasma following postsurgical bacteremia. Am J Physio/ 266, L689-L697

72. Henderson, B., Nair, S., Pallas, J., and Williams, M. A. (2011) Fibronectin: a multidomain host adhesin targeted by bacterial fibronectin-binding proteins. FEMS Microbiol. Rev. 35, 147-200

73. Cameron, C. E., Brown, E. L., Kuroiwa, J. M. Y., Schnapp, L. M., and Brouwer, N. L. (2004) Treponema pallidum fibronectin-binding proteins. J. Bacteriol. 186, 7019-7022

74. Schulze-Koops, H., Burkhardt, H., Heesemann, J., Kirsch, T., Swoboda, B., Bull, C., Goodman, S., and Emmrich, F. (1993) Outer membrane protein YadA of enteropathogenic yersiniae mediates specific binding to cellular but not plasma fibronectin. Infect. Immun. 61, 2513-2519

75. Heise, T., and Dersch, P. (2006) Identification of a domain in Yersinia virulence factor YadA that is crucial for extracellular matrix-specific cell adhesion and uptake. Proc. Natl. Acad. Sci. U. S. A. 103, 3375-3380

76. Frick, I. M., Crossin, K. L., Edelman, G. M., and Björck, L. (1995) Protein H-a bacterial surface protein with affinity for both immunoglobulin and fibronectin type III domains. EMBO J. 14, 16741679

77. Herrera, A. L., Faal, H., Moss, D., Addengast, L., Fanta, L., Eyster, K., Huber, V. C., and Chaussee, M. S. (2018) The Streptococcus pyogenes fibronectin/tenascin-binding protein PrtF.2 contributes to virulence in an influenza superinfection. Sci. Rep. 8, 12126

78. Hemperly, J. J., Murray, B. A., Edelman, G. M., and Cunningham, B. A. (1986) Sequence of a cDNA clone encoding the polysialic acid-rich and cytoplasmic domains of the neural cell adhesion molecule N-CAM. Proc. Natl. Acad. Sci. U. S. A. 83, 3037-3041

79. Kreikemeyer, B., Oehmcke, S., Nakata, M., Hoffrogge, R., and Podbielski, A. (2004) Streptococcus pyogenes Fibronectin-binding protein F2: expression profile, bindingcharacteristics, and impact on eukaryotic cell interactions. J. Biol. Chem. 279, 15850-15859

80. Bachert, B. A., Choi, S. J., LaSala, P. R., Harper, T. I., McNitt, D. H., Boehm, D. T., Caswell, C. C., Ciborowski, P., Keene, D. R., Flores, A. R., Musser, J. M., Squeglia, F., Marasco, D., Berisio, R., and Lukomski, S. (2016) Unique footprint in the scl1.3 locus affects adhesion and biofilm formation of the invasive M3-type group A Streptococcus. Front Cell Infect Microbio/ 6, 90

81. Erez, N., Truitt, M., Olson, P., Arron, S. T., and Hanahan, D. (2010) Cancer-associated fibroblasts are activated in incipient neoplasia to orchestrate tumor-promoting inflammation in an NF-kBdependent manner. Cancer Cell 17, 135-147

82. Kalluri, R. (2016) The biology and function of fibroblasts in cancer. Nature Rev. Cancer 16, 582

83. Burdick, C. G. (1937) William Bradley Coley 1862-1936. Ann. Surg. 105, 152-155

84. Decker, W. K., da Silva, R. F., Sanabria, M. H., Angelo, L. S., Guimarães, F., Burt, B. M., Kheradmand, F., and Paust, S. (2017) Cancer immunotherapy: historical perspective of a clinical revolution and emerging preclinical animal models. Front. Immunol. 8, 829-829 
85. Kilian, O., Dahse, R., Alt, V., Zardi, L., Hentschel, J., Schnettler, R., and Kosmehl, H. (2008) mRNA expression and protein distribution of fibronectin splice variants and high-molecular weight tenascin- $C$ in different phases of human fracture healing. Calcif. Tissue Int. 83, 101-111

86. Kilian, O., Dahse, R., Alt, V., Zardi, L., Rosenhahn, J., Exner, U., Battmann, A., Schnettler, R., and Kosmehl, H. (2004) Expression of EDA+ and EDB+ fibronectin splice variants in bone. Bone 35, 1334-1345

87. Sun, Z., Schwenzer, A., Rupp, T., Murdamoothoo, D., Vegliante, R., Lefebvre, O., Klein, A., Hussenet, T., and Orend, G. (2018) Tenascin-C Promotes Tumor Cell Migration and Metastasis through Integrin a9ß1-Mediated YAP Inhibition. Cancer Res. 78, 950-961

88. Hoe, N. P., Nakashima, K., Lukomski, S., Grigsby, D., Liu, M., Kordari, P., Dou, S.-J., Pan, X., Vuopio-Varkila, J., Salmenlinna, S., McGeer, A., Low, D. E., Schwartz, B., Schuchat, A., Naidich, S., De Lorenzo, D., Fu, Y.-X., and Musser, J. M. (1999) Rapid selection of complement-inhibiting protein variants in group A Streptococcus epidemic waves. Nat. Med. 5, 924-929

89. Caswell, C. C., Lukomska, E., Seo, N. S., Höök, M., and Lukomski, S. (2007) Scl1-dependent internalization of group A Streptococcus via direct interactions with the $\alpha 2 \beta 1$ integrin enhances pathogen survival and re-emergence. Mol. Microbiol. 64, 1319-1331

90. Cramer, T., Yamanishi, Y., Clausen, B. E., Förster, I., Pawlinski, R., Mackman, N., Haase, V. H., Jaenisch, R., Corr, M., Nizet, V., Firestein, G. S., Gerber, H. P., Ferrara, N., and Johnson, R. S. (2003) HIF-1 $\alpha$ is essential for myeloid cell-mediated inflammation. Cell 112, 645-657

91. Humtsoe, J. O., Kim, J. K., Xu, Y., Keene, D. R., Höök, M., Lukomski, S., and Wary, K. K. (2005) A streptococcal collagen-like protein interacts with the $\alpha 2 \beta 1$ integrin and induces intracellular signaling. J. Biol. Chem. 280, 13848-13857

92. Caswell, C. C., Han, R., Hovis, K. M., Ciborowski, P., Keene, D. R., Marconi, R. T., and Lukomski, S. (2008) The Scl1 protein of M6-type group A Streptococcus binds the human complement regulatory protein, factor $\mathrm{H}$, and inhibits the alternative pathway of complement. Mol. Microbiol. 67, 584-596

93. Kelsh, R., You, R., Horzempa, C., Zheng, M., and McKeown-Longo, P. J. (2014) Regulation of the innate immune response by fibronectin: synergism between the III-1 and EDA domains. PLoS One 9, e102974

94. Aukhil, I., Joshi, P., Yan, Y., and Erickson, H. P. (1993) Cell- and heparin-binding domains of the hexabrachion arm identified by tenascin expression proteins. J Biol Chem 268, 2542-2553

95. Alva, V., Nam, S. Z., Söding, J., and Lupas, A. N. (2016) The MPI bioinformatics toolkit as an integrative platform for advanced protein sequence and structure analysis. Nucleic Acids Res. 44, W410-415

96. Van Der Spoel, D., Lindahl, E., Hess, B., Groenhof, G., Mark, A. E., and Berendsen, H. J. (2005) GROMACS: fast, flexible, and free. J. Comput. Chem. 26, 1701-1718 
97. Kumar, D., New, J., Vishwakarma, V., Joshi, R., Enders, J., Lin, F., Dasari, S., Gutierrez, W. R., Leef, G., Ponnurangam, S., Chavan, H., Ganaden, L., Thornton, M. M., Dai, H., Tawfik, O., Straub, J., Shnayder, Y., Kakarala, K., Tsue, T. T., Girod, D. A., Van Houten, B., Anant, S., Krishnamurthy, P., and Thomas, S. M. (2018) Cancer-associated fibroblasts drive glycolysis in a targetable signaling loop implicated in head and neck squamous cell carcinoma progression. Cancer Res. 78, 3769-3782

98. Oliver-Kozup, H. A., Elliott, M., Bachert, B. A., Martin, K. H., Reid, S. D., Schwegler-Berry, D. E., Green, B. J., and Lukomski, S. (2011) The streptococcal collagen-like protein-1 (Scl1) is a significant determinant for biofilm formation by group A Streptococcus. BMC Microbiol. 11, 262 
Figure 1

\section{FIGURES}

A
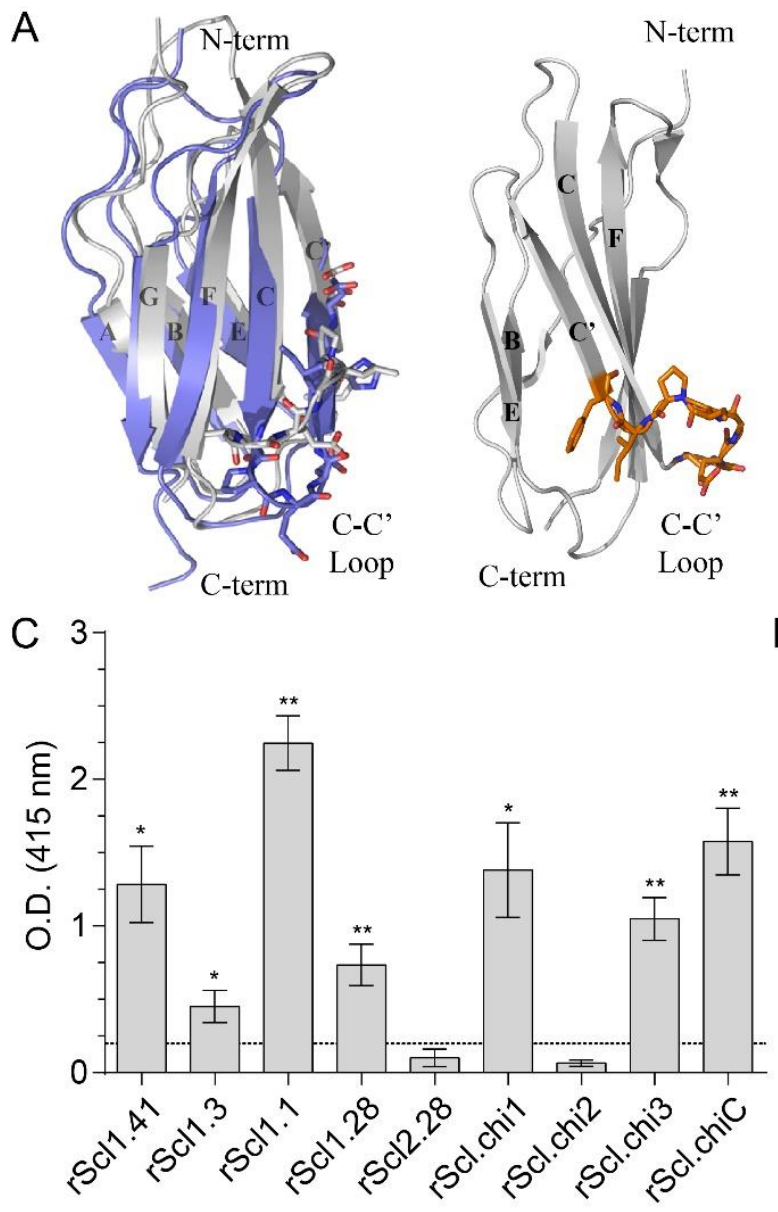

E

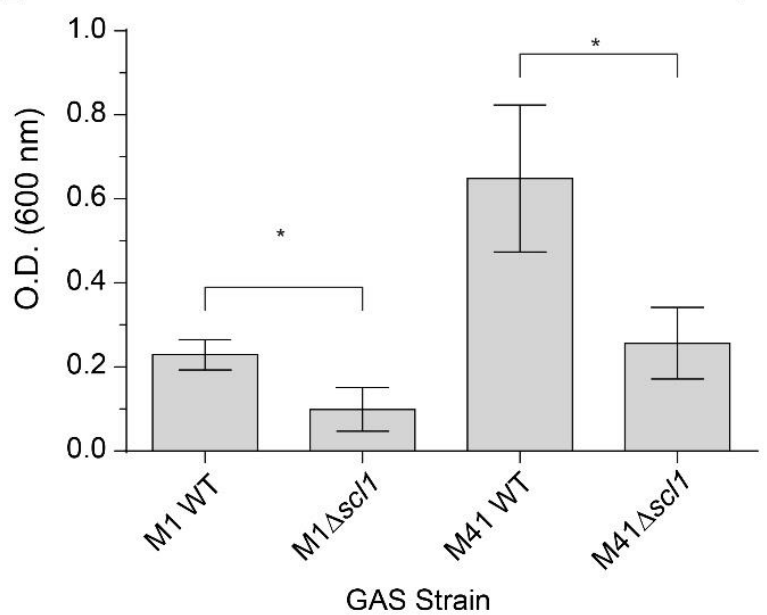

F
B

\begin{tabular}{|c|c|c|c|}
\hline J & & $\mathrm{C}$ & \\
\hline a-Helix & Loop-region & a-Helix & $\begin{array}{l}\mathrm{rScl} 1.1-\mathrm{V} / \\
\mathrm{rScl} 128-\mathrm{V}\end{array}$ \\
\hline a-Helix & Loop-region & a-Helix & $\mathrm{rScl} 2.28-\mathrm{V}$ \\
\hline a-Helix & Loop-region & a-Helix & $\begin{array}{l}\text { rScl.chi1-V/ } \\
\text { rScl.chi3-V }\end{array}$ \\
\hline a-Helix & Loop-region & a-Helix & rScl.chi2 \\
\hline a-Helix & Loop-region & a-Helix & rScl.chiC \\
\hline
\end{tabular}

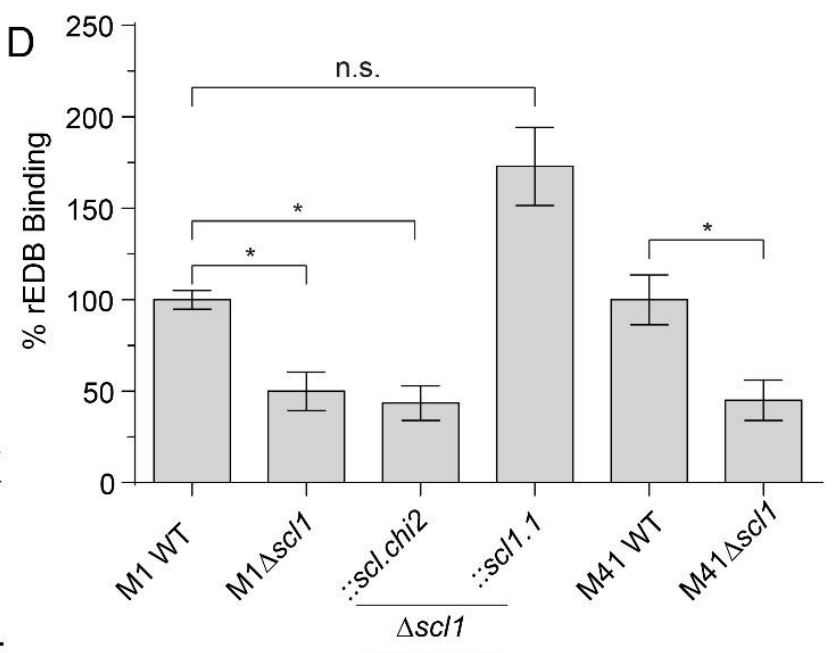
GAS Strain
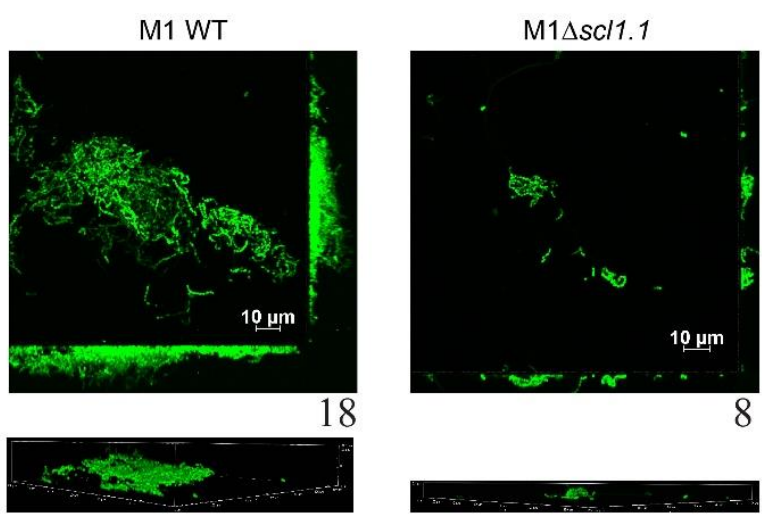
Figure 1. Scl1-EDB binding mediates GAS adherence and biofilm formation. Recombinant fibronectin type III repeat, extra domain $B(r E D B)$ was tested for binding to recombinant streptococcal collagen-like proteins $(\mathrm{rScl})$, as well to whole-GAS cell. Scl1-mediated biofilm formation was studied on rEDB coatings. $(A)$ Cartoon representations of the EDB and EDA domains of fibronectin. Structures of EDB (grey - adapted from PDB accession code: 5n48), and EDA (blue - adapted from PDB accession code: 1j8k) are shown in forward (left) and side (right) views. Left, front view of superimposed $\beta$-sandwich structures of EDA and EDB, with each $\beta$-strand marked (A, B, C, C', E, F, G) and C-C' loops shown in stick representation. Right, side view of EDB structure, depicting the $\mathrm{C}-\mathrm{C}^{\prime}$ loop in ball and stick representation. The $\mathrm{N}$ - and $\mathrm{C}$-terminal ends are labeled in both models. $(B)$ Schematic representation of the variable $(V)$ domains in recombinant Scl constructs used. Homotrimeric rScl1.1-, rScl1.28 (gray box), and rScl2.28-V domains (white box) consist of three conserved pairs of anti-parallel $\alpha$-helices, with connecting loops (19). Chimeric proteins were generated by replacing either the entire ( $r S c l . c h i 1-3)$ or partial (rScl.chiC) loop sequences between different constructs. (C) rEDB binding to naturally-derived and chimeric $\mathrm{rScl}$ constructs. $\mathrm{rScl}$ proteins were immobilized onto Strep-Tactin coated microplate wells and incubated with rEDB. Primary anti-His-tag mAb and HRP-conjugated secondary Ab were used for ligand detection by ELISA. Graph bars indicate the mean $\mathrm{OD}_{415 \mathrm{~nm}}$ normalized against BSA controls. Statistical analysis was calculated using Student's two-tailed $t$ test from three independent experiments, each performed in triplicate wells $(N=3 \pm S D) ;{ }^{*} P \leq 0.05,{ }^{* *} P \leq 0.01$. Statistical significance evaluates the differences in rEDB binding by naturally-derived rScl1 (left 4) and chimeric proteins (right 4), as compared to ECM-binding-negative rScl2.28 control protein; rEDB binding by rScl.chi2 was compared to that of rScl1.1. Dashed line indicate threshold $\mathrm{OD}_{415 \mathrm{~nm}}+2 \mathrm{SD}$ values recorded for binding-negative rScl2.28 control protein. (D) rEDB binding to whole GAS cells. Isogenic M1- and M41type GAS strains were used, as well as the M1 isogenic $\Delta$ scl1 mutant complemented for the expression of Scl1.1 ( $\Delta \mathrm{scl1}:: s c / 1.1)$ and Scl.chi2 ( $\Delta \mathrm{scl1}:: s c l . c h i 2)$ proteins. rEDB binding to whole GAS cells was detected by flow cytometry with primary anti-His-tag mAb; binding to GAS WT cells was set as $100 \%$. Statistical analysis was calculated using Student's two-tailed $t$-test from three independent experiments $(\mathrm{N}=3 \pm \mathrm{SD})$; ${ }^{*} \mathrm{P} \leq 0.05$. ( $E$ ) Assessment of biofilm formation on rEDB-coated surfaces. Isogenic M1- and M41-type GAS strains were compared. Biofilm formation was evaluated spectrophotometrically following crystal violet staining. Graphic bars indicate the mean $\mathrm{OD}_{600 \mathrm{~nm}}$ normalized against BSA controls. Statistical analysis was calculated using Student's two-tailed $t$-test from three independent experiments $(\mathrm{N}=3 \pm \mathrm{SD})$; ${ }^{*} \mathrm{P} \leq 0.05$. $(F)$ Microscopy imaging of GAS biofilms formed on rEDB coating. GFP-expressing M1 WT and $\Delta s c / 1.1$ mutant strains were grown on rEDB-coated glass coverslips for $24 \mathrm{~h}$. Two-dimensional orthogonal views of GAS biofilms are representative of $Z$ stacks from 10 fields within a single experiment. Average vertical thickness is indicated in micrometers below two-dimensional orthogonal views, taken from 10 arbitrary fields per experiment. 
Figure 2

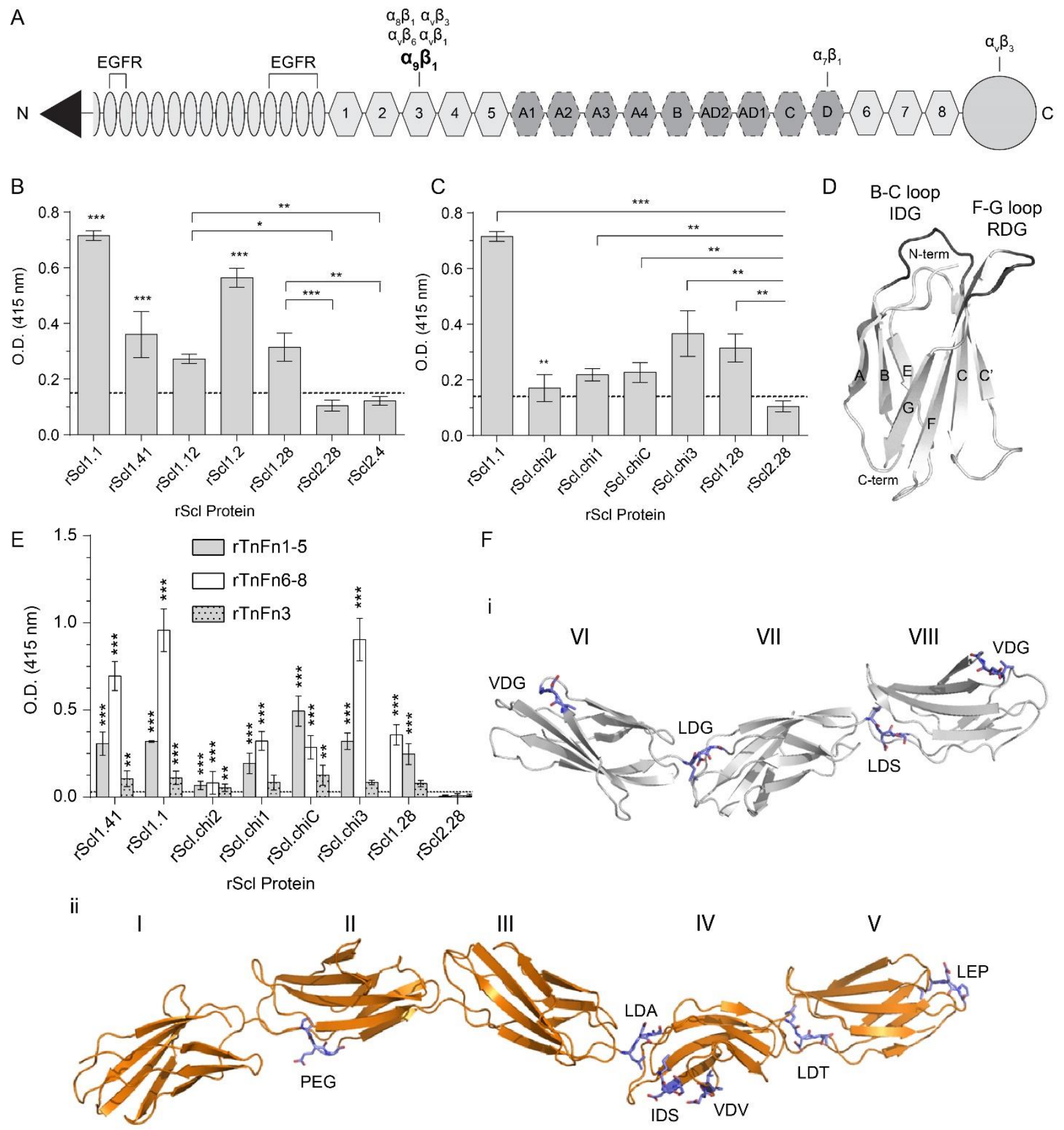

Figure 2. Scl1 of GAS binds to the fibronectin type III repeats in tenascin $\mathrm{C}(\mathrm{TnC})$. For ligand binding by ELISA, rScl proteins were immobilized onto Strep-Tactin coated microplate wells and incubated with full-

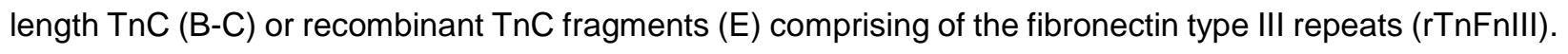
Primary anti-ECM mAb and HRP-conjugated secondary Ab were used for ligand detection. Graph bars 
indicate the mean $\mathrm{OD}_{415 \mathrm{~nm}}$ normalized against BSA controls. Statistical analysis was calculated using Student's two-tailed $t$-test from three independent experiments, each performed in triplicate wells $(\mathrm{N}=3 \pm \mathrm{SD}) ;{ }^{*} \mathrm{P} \leq 0.05,{ }^{* *} \mathrm{P} \leq 0.01,{ }^{* * *} \mathrm{P} \leq 0.001$. Statistical significance evaluates the differences in $\mathrm{TnC}$ and rTnFn binding by rScl1 and chimeric proteins, as compared to ECM-binding-negative rScl2.28 control protein; $\mathrm{TnC}$ and $\mathrm{rTnFn}$ binding by rScl.chi2 was compared to that of rScl1.1. Dashed line indicate threshold $\mathrm{OD}_{415 \mathrm{~nm}}+2 \mathrm{SD}$ values recorded for binding-negative $\mathrm{rScl} 2.28$ control protein. $(A)$ Schematic representation of full-length $\mathrm{TnC}$. Depicted are from the N-terminus: assembly domain (triangle), epidermal growth factorlike repeats (ovals), constitutively expressed fibronectin type III repeats 1-5 and 6-8 (light hexagons), alternatively spliced fibronectin type III repeats (dark hexagons), fibrinogen-related domain (circle). Known integrin-binding domains are marked above the model. (B) TnC binding to recombinant Scl1- and Scl2derived constructs. rScl panel represents diverse Scl1 and Scl2 variants originating from strains of several M-types. (C) TnC binding to $\mathrm{rScl}$ chimeric proteins. $\mathrm{rScl}$ panel represents constructs shown in Fig. 1B-C and described (19). (D) Cartoon representation of the third fibronectin type III repeat of TnC PDB accession code: 1TEN). $\beta$-strands of the EDB $\beta$-sandwich are labelled (A, B, C, C', D, E, F, G); the B-C loop with LDV motif and the F-G loop harboring the RGD sequence is shown in dark gray. $(E$ ) $r S c l$ binding to recombinant fibronectin type III fragments within TnC. rScl panel represents constructs shown in Fig. 1B-C above and previously described (19). ( $F$ ) Cartoon representations of the homology models of the fibronectin type III repeats 1-5 (orange) and 6-8 (gray) of TnC. Models of (I) TnFn6-8 repeats, and (ii) TnFn1-5 repeats. Segments harboring the potential LDV-like Scl1-binding motifs are shown in light blue ball and stick representation. Model of TnFn1-5 based on PDB accession code 3t1w; model of TnFn6-8 based on PDB accession code $3 r 8 q$. 


\section{Figure 3}

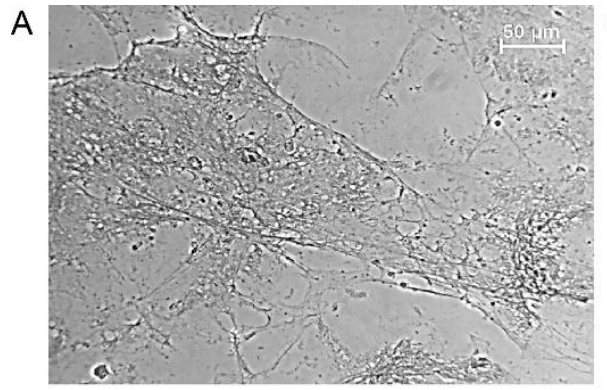

B

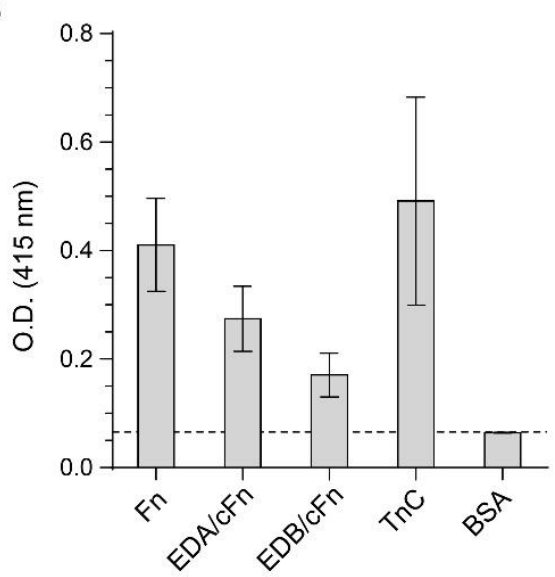

C
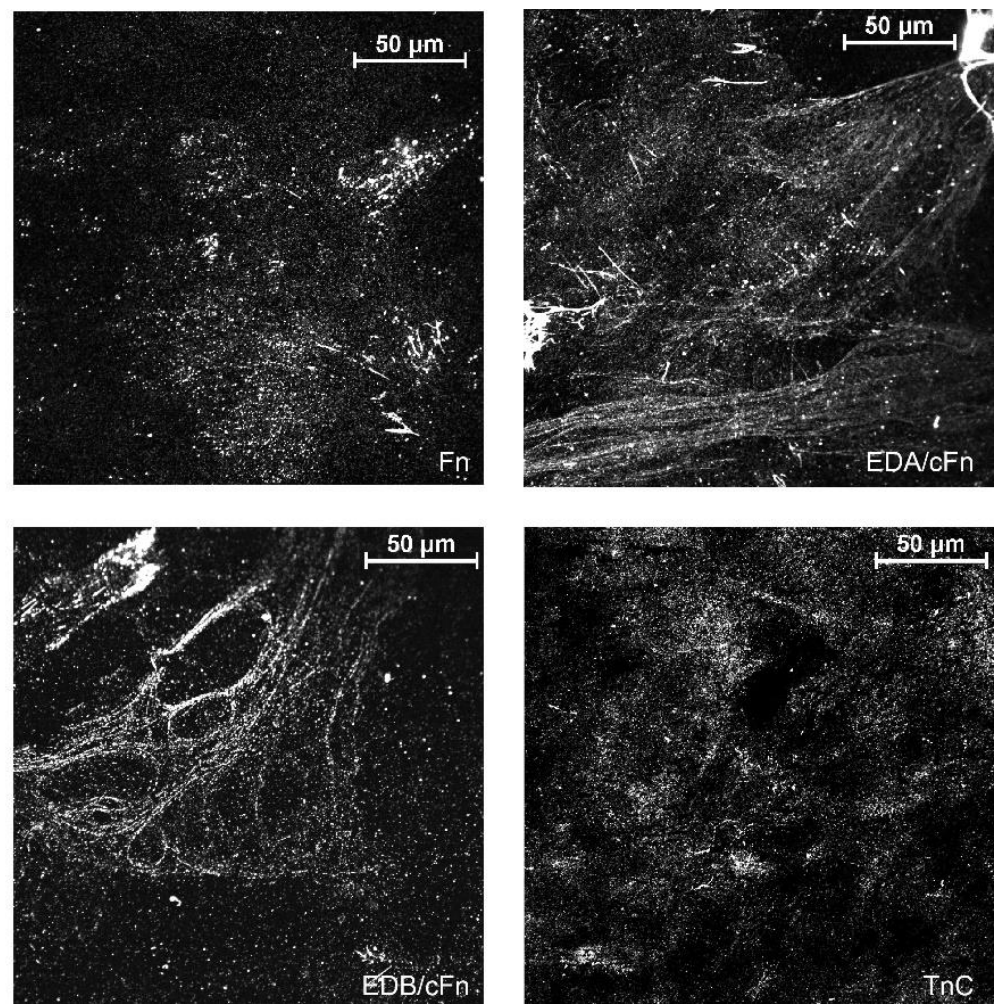

Figure 3. Characterization of extracellular matrices deposited by cancer-associated fibroblasts (CAFs). CAFs were isolated from a stage IV laryngeal primary tumor and grown to confluency; matrices were prepared after the removal of cells by treatment with EGTA. Matrices were evaluated for the presence of EDA- and EDB-containing fibronectins and TnC. $(A)$ Visualization of the overall structure of ECM deposited by CAFs. Ponceau $S$ staining reveals complex fibrillary network of the matrices used in this study. $(B)$ Characterization of the ECM deposited by CAFs by ELISA. The presence of total cFn, EDA/cFn, EDB/cFn, and $\mathrm{TnC}$ was assessed with specific mAbs and secondary HRP-conjugated antibody. Graph bars indicate the mean $\mathrm{OD}_{415 \mathrm{~nm}}$. Three independent experiments were performed, each with triplicate wells $(\mathrm{N}=3 \pm S D)$. Dashed line indicate threshold $\mathrm{OD}_{415 \mathrm{~nm}}+2 \mathrm{SD}$ values recorded for BSA control wells. $(C)$ Immunofluorescent visualization of the ECM deposited by CAFs. CAFs were grown on glass coverslips, then cells were removed from matrices and incubated with primary mAbs specific for cFn, EDA/cFn, EDB/cFn, and TnC, followed by secondary Ab conjugated with Alexa Fluor® 568. Images were taken using confocal microscope with 60x objective; representative images are shown from 2 independent experiments, imaging 10 arbitrary fields per coverslip. 


\section{Figure 4}
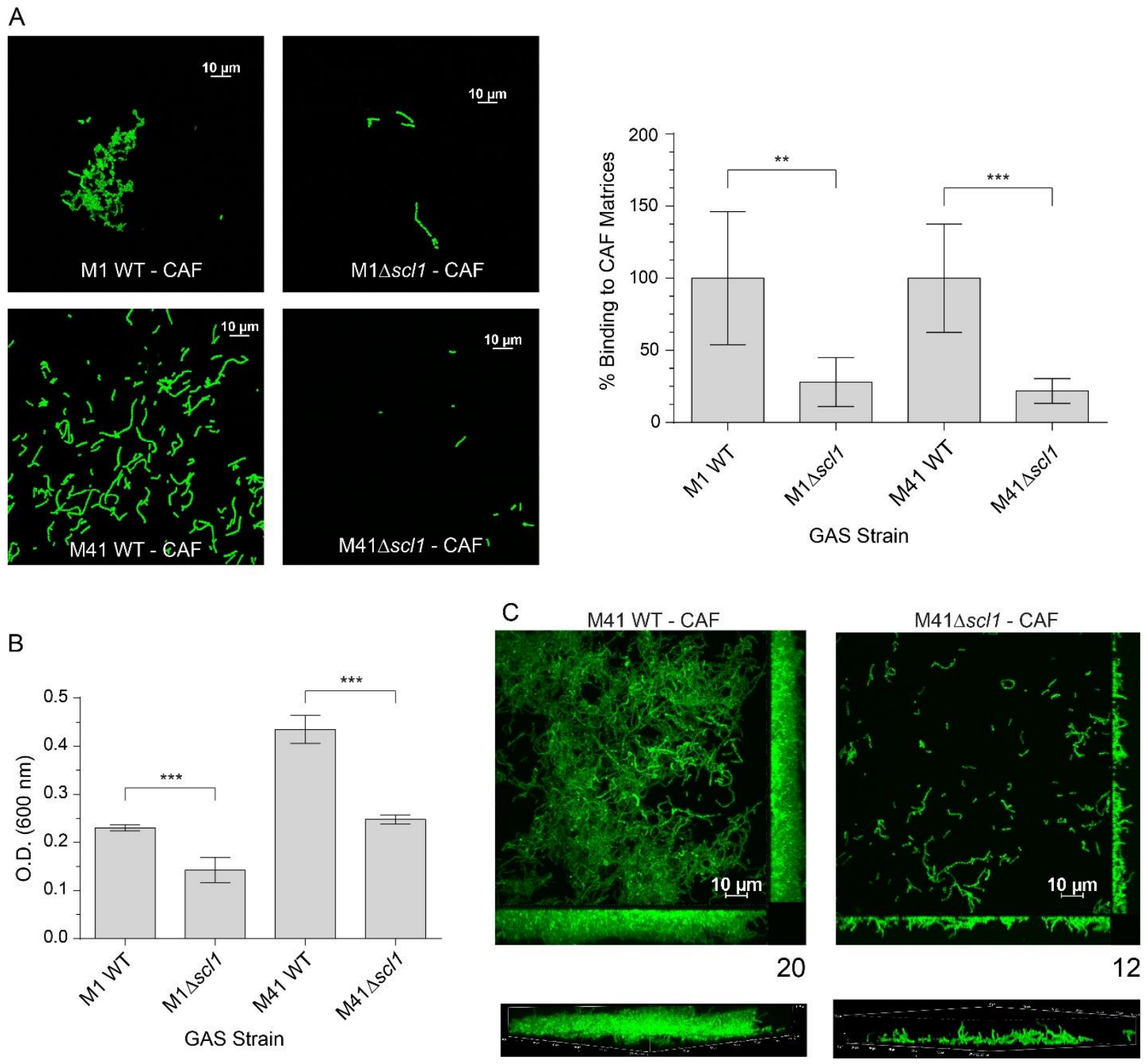

Figure 4. Scl1-mediated GAS attachment to and biofilm formation on ECM deposited by cancer-derived fibroblasts. Wild type (WT) GAS strains M1 and M41, and their isogenic $\Delta s c / 1$ mutants were compared for attachment and biofilm formation. (A) GAS adherence to CAF-derived ECM. Isogenic GFP-GAS strains were inoculated onto CAF-derived ECM coatings, allowed to attach for 1 hour, and imaged using fluorescent confocal microscope with 100x objective. Left, representative images of attached GAS were taken in 10 fields, over three independent experiments. Right, quantification of GAS attachment with WT binding set as a 100\%; bacteria were counted in 10 fields, and the average from all 10-fields was calculated. 
Statistical significance was calculated using Student's two-tailed $t$-test from three independent experiments, each performed in duplicate wells $(N=3 \pm S D) ;{ }^{* *} P \leq 0.01,{ }^{* * *} P \leq 0.001$. Statistical analysis evaluates the difference between adherence to CAF-derived matrices by the WT and their respective isogenic $\Delta s c / 1$ mutants. (B) Assessment of biofilm formation on CAF matrices. Isogenic M1- and M41-type GAS strains were compared. Biofilm formation was evaluated spectrophotometrically following crystal violet staining. Graphic bars indicate the mean $\mathrm{OD}_{600 \mathrm{~nm}}$ normalized against BSA controls. Statistical analysis was calculated using Student's two-tailed $t$-test from three independent experiments $(\mathrm{N}=3 \pm S D)$; ${ }^{*} \leq 0.05$. (C) Microscopy imaging of GAS biofilms formed on CAF matrices. GFP-expressing M1 WT and $\Delta \mathrm{scl1.1}$ mutant strains were grown for $24 \mathrm{~h}$ on CAF matrices coated glass coverslips. Two-dimensional orthogonal views of GAS biofilms are representative of $Z$ stacks from 10 fields within a single experiment. Average vertical thickness is indicated in micrometers below two-dimensional orthogonal views, taken from 10 arbitrary fields per experiment. 
Figure 5

A

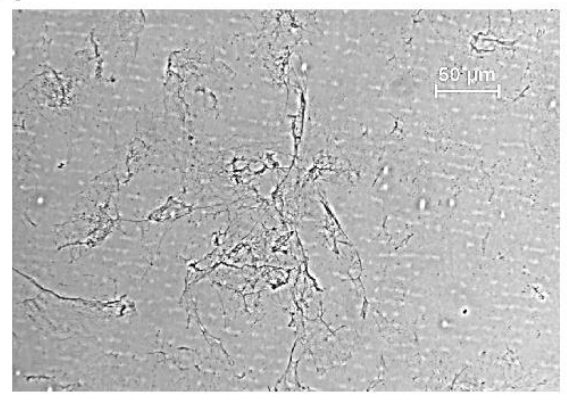

B

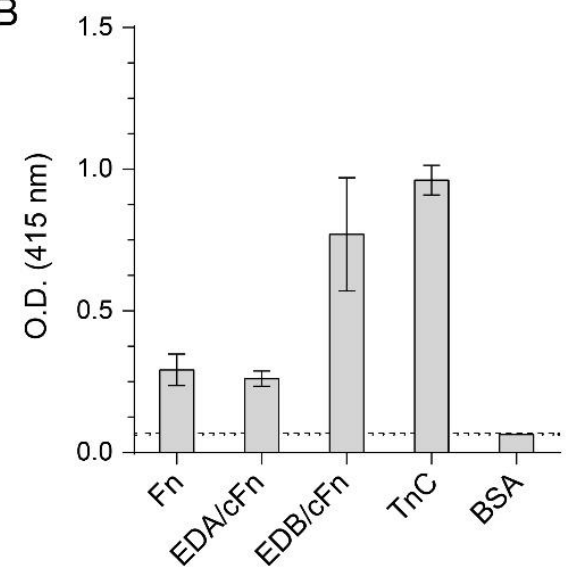

C
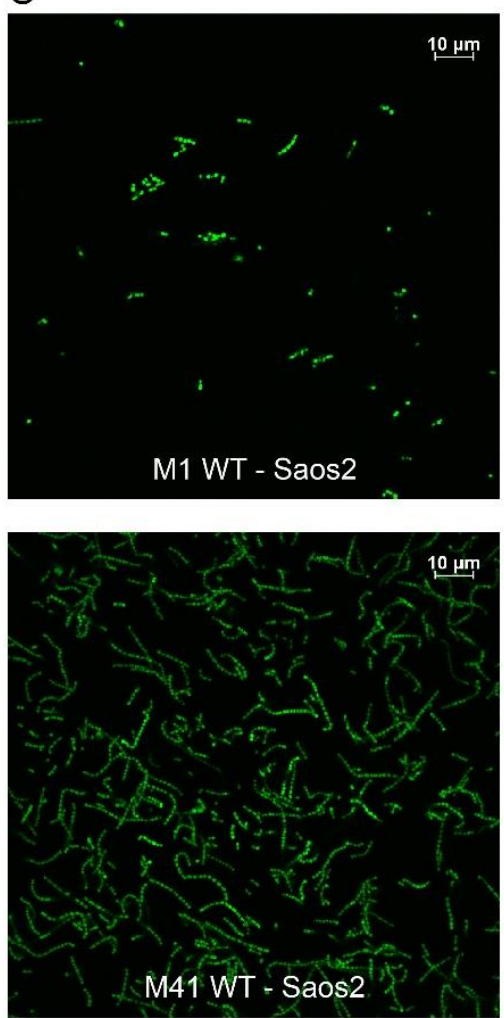

M41 WT - Saos2
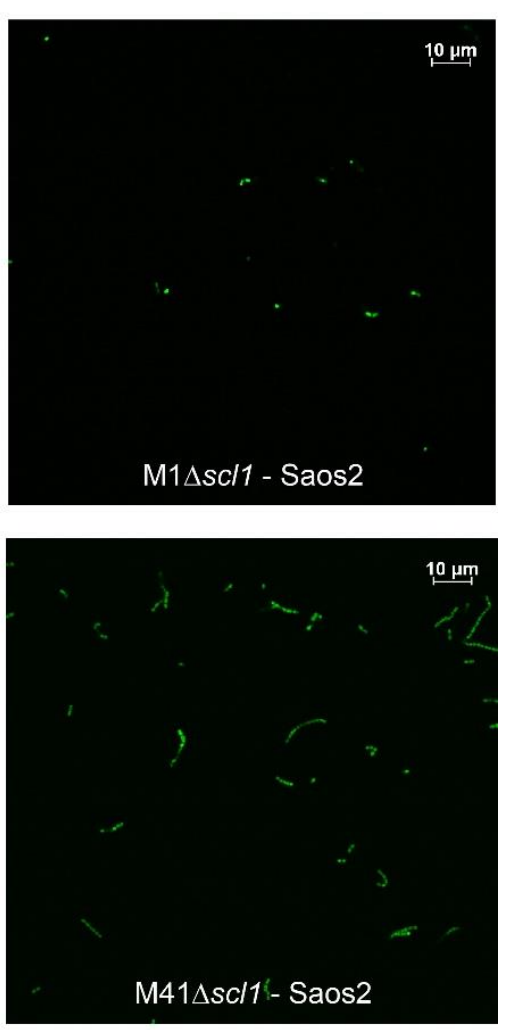

$\mathrm{M} 41 \Delta \mathrm{sc} / 1$ - Saos2

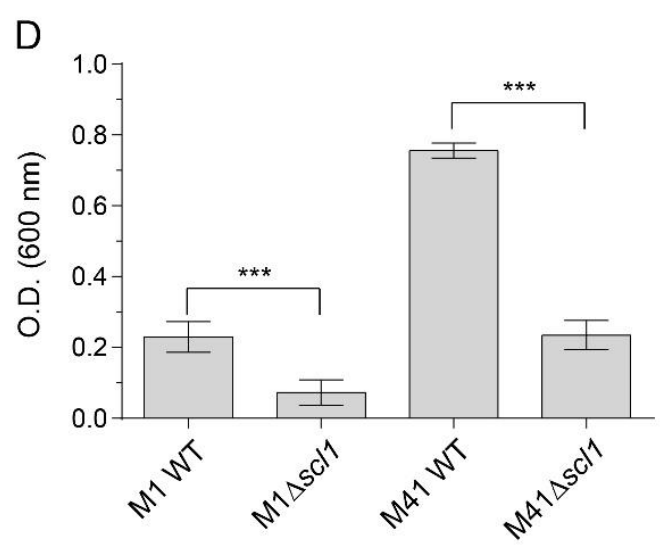

GAS Strain

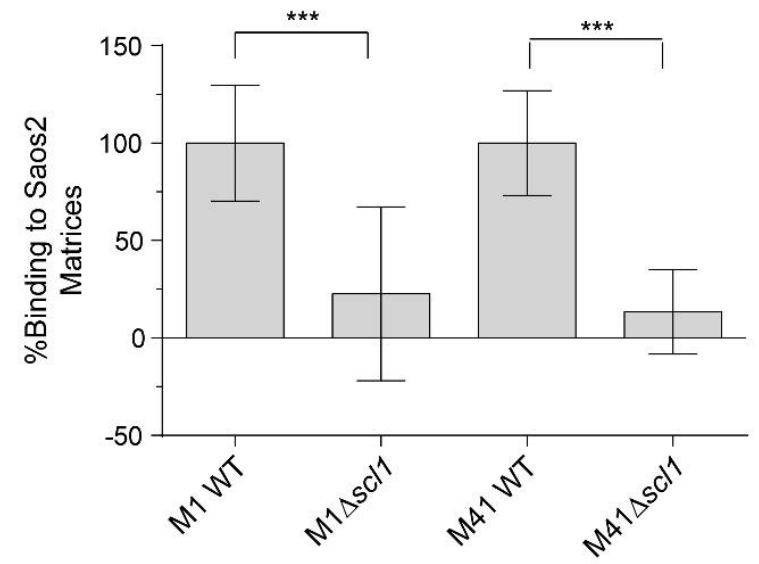

GAS Strain

Figure 5. Scl1-mediated GAS attachment to and biofilm formation on ECM deposited by bone osteosarcoma cells. Wild type (WT) GAS strains M1 and M41, and their isogenic $\Delta s c / 1$ mutants were compared for attachment and biofilm formation on the ECM produced by osteosarcoma Saos-2 cells. $(A)$ Representative image of Ponceau $S$ staining of ECM network deposited by Saos-2 cells. (B) 
Characterization of the ECM deposited by Saos-2 cells by ELISA. The presence of total cFn, EDA/cFn, $\mathrm{EDB} / \mathrm{cFn}$, and TnC was assessed with specific $\mathrm{mAbs}$ and secondary HRP-conjugated antibody. Graph bars indicate the mean $\mathrm{OD}_{415 \mathrm{~nm}}$. Three independent experiments were performed, each with triplicate wells $(\mathrm{N}=3 \pm S D)$. Dashed line indicate threshold $\mathrm{OD}_{415 \mathrm{~nm}}+2 \mathrm{SD}$ values recorded for BSA control wells. $(C)$ GAS attachment on Saos-2-derived ECM. Isogenic GFP-GAS strains were inoculated onto Saos-2-derived matrices, allowed to attach for 1 hour, and imaged using fluorescent confocal microscope with 100x objective. Top, representative images of attached GAS were taken in 10 fields, over three independent experiments. Bottom, quantification of GAS attachment with WT binding set as a $100 \%$; bacteria were counted in 10 fields, and the average from all 10-fields was calculated. Statistical analysis was calculated using Student's two-tailed $t$-test from three independent experiments, each performed in duplicate wells $(N=3 \pm S D) ;{ }^{* *} P \leq 0.01,{ }^{* *} P \leq 0.001$. Statistical significance evaluates the difference between adherence to Saos-2-derived matrices by the WT and their respective isogenic $\Delta s c / 1$ mutants. (D) GAS biofilm formation on Saos-2-derived ECM. Isogenic GAS strains were inoculated onto Saos-2-derived matrices and grown for 24 hours. Bacterial biomass was evaluated spectrophotometrically following crystal violet staining. Graphic bars indicate the mean $\mathrm{OD}_{600 \mathrm{~nm}}$ normalized against BSA controls. Statistical analysis was calculated using Student's two-tailed $t$-test from three independent experiments $(N=3 \pm S D) ;{ }^{* *} \mathrm{P} \leq 0.001$. 
Figure 6

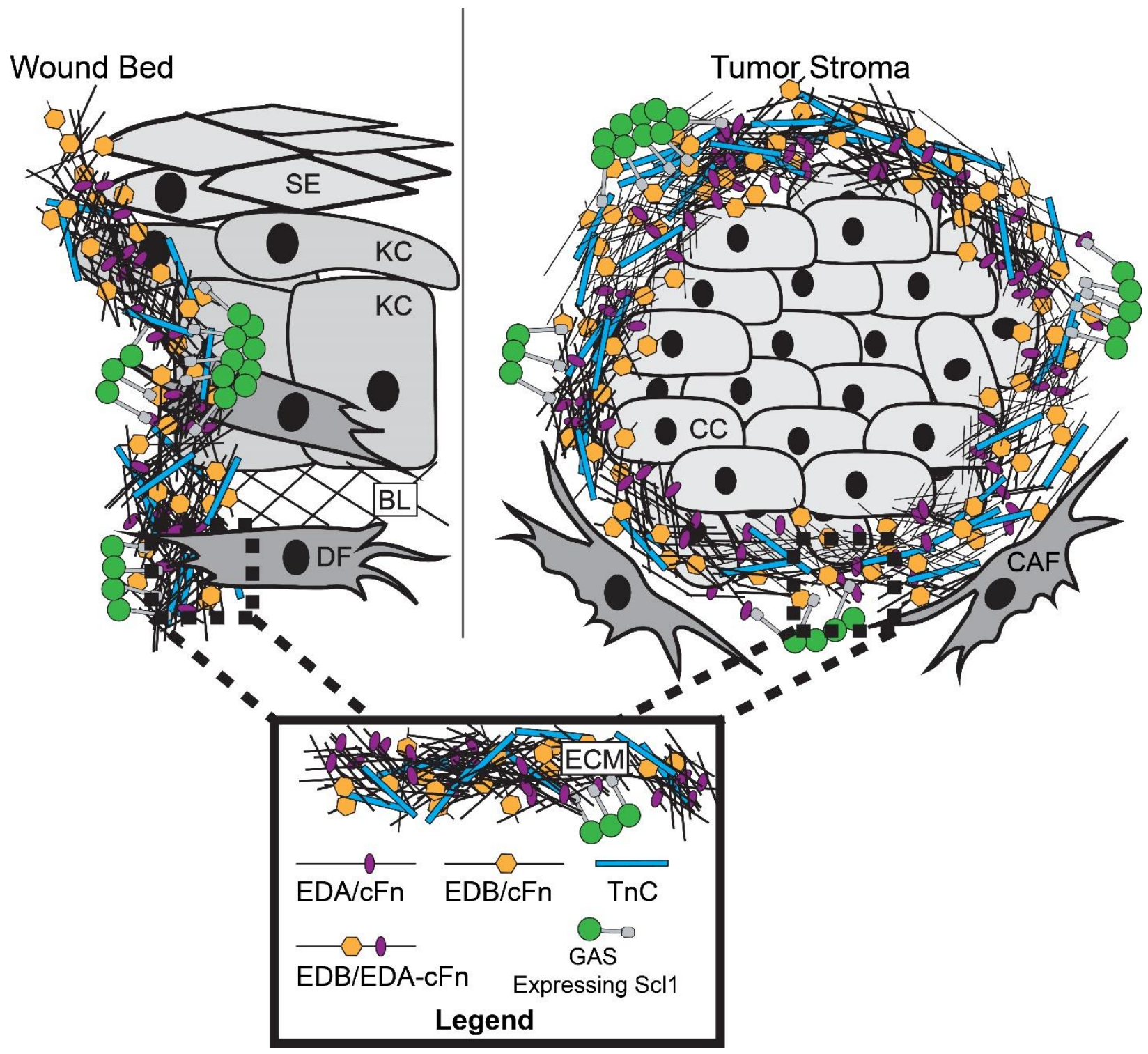

Figure 6. Model of GAS colonization of wound and tumor microenvironments. The wound and tumor microenvironments are enriched in isoforms of cellular fibronectin (cFn) that contain extra domain $A$ (EDA) and extra domain $B(E D B)$, as well as monomer of tenascin-C (TnC). Left, GAS gains access to the host via portal of entry, such as through a breach in keratinized squamous epithelium (SE), into a tissue environment that contains keratinocytes (KC), basal lamina (BL) ECM, and dermal fibroblasts (DF). Within wound, cells such as DFs deposit cFn isoforms that contain EDA and EDB, as well as TnC. GAS-Scl1 adhesin binds EDA and EDB of $\mathrm{cFn}$, and $\mathrm{TnC}$ within the wound, promoting wound colonization. Right, Cancer cells (CC) are surrounded by cancer-associated fibroblasts (CAF), which deposit cFn isoforms that 
contain EDA and/or EDB, and TnC, recognized by GAS-Scl1. Enlarged insert, close-up view of the woundand tumor-associated ECM 


\section{DISCUSSION AND PROSPECTS}

Our work sought to investigate the interactions between the Scl1 adhesin of GAS and wound-associated ECM, and the contribution of these interactions to GAS adherence and host colonization. The work presented in detail within preceding chapters elaborates on important structural determinants and mechanisms of binding for Scl1 and identifies FnIII repeats that are associated with the wound microenvironment, as Scl1 targets. In this chapter, we will selectively discuss our research in the context of important implications, resulting from Scl1-Fnlll binding, in GAS wound colonization and pathogenesis, GAS targeting, and application to cancer therapies.

\section{Scl1 is a unique Fn-binding adhesin of GAS that adapted to colonize wound microenvironment}

Classical fibronectin-binding proteins of GAS, such as proteins F1 and F2, contain a block of fibronectin-binding repeats that recognize and bind to Fn type I repeats present in both pFn and cFn (1). These classical Fn-binding adhesins of GAS bind FnI repeats via a $\beta$ zipper mechanism, wherein the Fn-binding repeats adopt a $\beta$-strand conformation and then align parallel to the outer most $\beta$-strand within the Fnl structure (2). Scl1 lacks these canonical Fn-binding repeats and binds Fnlll repeats through a different mechanism. Scl1 interacts with EDA, EDB, and TnFnlll via surface-exposed guiding loops of the Scl1-V domain that surround a negatively-charged cleft bordered by a crown of positive and neutral surface charges present in binding-positive Scl1 proteins but not in bindingnegative Scl2 proteins (3). In this regard, Scl1 is similar to streptococcal adhesins, CshA of $S$. gordonii and BspA of $S$. agalactiae, that exploit a "catch-clamp" Fn-binding mechanism $(4,5)$. In "catch-clamp" binding model, a surface-exposed loop caps the binding cleft, and guides Fn recognition towards binding cleft. The "catch-clamp" adhesins and Scl1-V domain share similar electrostatic surface charge distributions. Future crystallization of Scl1 in complex with Fnlll ligand will aim to verify binding mechanism.

Scl1 variants from several different M-types of GAS were shown to selectively bind cFn, but not pFn (6), and our initial work showed that Scl1 binds EDA (7). Here, I identified that Scl1 also binds to EDB of cFn, as well as to Fnlll repeats of TnC. Two other GAS 
proteins have been reported to bind to the FnIII repeats of $\mathrm{TnC}$ and $\mathrm{Fn}$ : the protein $\mathrm{H}$, and protein $\mathrm{F} 2$. The $\mathrm{M}$-like protein $\mathrm{H}$ binds factor $\mathrm{H}$ and $\mathrm{IgG}$, as well as the neural cell adhesion molecule (N-CAM), which contains Fnlll repeats (8). Protein $\mathrm{H}$ binding to $\mathrm{N}-\mathrm{CAM}$ was competitively inhibited with $\mathrm{pFn}$ fragments encompassing the FnIII repeat region, as well as with two different Fnlll-containing recombinant proteins derived from TnC (9). Protein F2 contains the prototypical Fn-binding repeats $(10,11)$. To our knowledge, direct binding to the Fnlll repeats in either Fn or TnC by F2 protein has not been reported. We, on the contrary, showed direct binding between rScl1 and rFnlll. Scl1 is, therefore, the only GAS adhesin with demonstrated binding to EDA, EDB, and to the TnFnlll repeats, which promotes GAS attachment and biofilm development within the wound. Additional bacterial adhesins may also bind to EDA and/or EDB, like Tp0155 of Treponema pallidum and YadA of Yersinia spp. (12). The outer envelope protein Tp0155 of T. pallidum displayed a selective binding to $\mathrm{cFn}$ (referred to in text as insoluble matrix fibronectin), but not plasma $\mathrm{pFn}$, which contributed to $T$. pallidum host-cell adhesion (13). YadA of $Y$. pseudotuberculosis and $Y$. enterocolitica also demonstrated preferential binding to cFn, promoting bacterial cell entry $(14,15)$. Further investigations are needed to assess $\mathrm{EDA} / \mathrm{cFn}$ and/or EDB/cFn binding by these adhesins.

\section{EDA/EDB cFn isoforms and TnC are expressed during wound healing and are accessible to Scl1}

Immediately after the initial wounding (portal of entry), platelets deposit EDA and/or EDB containing $\mathrm{cFn}$ isoforms within the wound, as a component of the initial clot and first provisional matrix during wound healing. These isoforms of $\mathrm{cFn}$ are released from granules that are formed during the development of platelets from megakaryocytes (16). In addition, TnC circulates at low levels in human blood, and is deposited within the initial matrix formed during clotting $(17,18)$. Several hours after wounding, fibroblasts and endothelial cells begin to deposit large amounts of EDA- and/or EDB-containing cFn isoforms, and $\mathrm{TnC}$, within the wound bed as a transitional provisional matrix, contributing to proper wound healing (19); macrophages have also been shown to release EDA containing $\mathrm{cFn}$ within the wound bed to signal pro-inflammatory cytokine release (20). These isoforms of $\mathrm{cFn}$ are present within healing tissue for up to 2 weeks 
after wound is healed (19). As we have shown previously (7), and outlined above, Scl1 binds to EDA and EDB isoforms of $\mathrm{cFn}$, and to all splice-variants of $\mathrm{TnC}$ via the constitutively-expressed Fnlll repeats of TnC. This may enable GAS to colonize the immediate wound area (Fig. 1), rather than become invasive and disseminate to other tissues. As an example, the M3-type GAS strains that are associated with invasive infections $(21,22)$ lack surface Scl1, due to a null mutation within collagen domain, which prevents cellular attachment (23). Restoring Scl1-surface expression enables Scl1mediated ECM binding in these strains, enhances biofilm formation on ECM coated surfaces (24), and decreases overall virulence (25). Therefore, the capacity of Scl1 to recognize multiple targets within wound microenvironment augments GAS colonization, while decreasing invasive spread and avoiding killing the host. In addition to adhesion, we have shown that the Scl1-EDA and -EDB interaction contributes to GAS biofilm formation in vitro ((7), current work). GAS biofilms are found in both non-invasive and invasive infections $(26,27)$, and are thought to play an immune evasive role and offer protection from antibiotics $(28,29)$. Therefore, the Scl1 capacity to recognize and bind $\mathrm{EDA} /$ and $\mathrm{EDB} / \mathrm{cFn}$, and $\mathrm{TnC}$ within the wound facilitates enhanced tissue-microcolony formation (Fig. 1), thus, contributing to the pathogenesis of GAS infections. To examine the importance of EDA/cFn, EDB/cFn, and TnC in GAS wound-colonization, both in vitro and in vivo studies can be conducted. Using cell-derived matrices or organotypic cultures with EDA, EDB, TnC, or combinations of the three, could be used to assess changes in GAS colonization without these components. Inasmuch as double EDA/EDB-knockouts are embryonic-lethal, single knockout mouse lines exist for both EDA and EDB, as well as whole TnC knockout animals (30-32), enabling the study of EDA, EDB, and TnC during GAS wound infections, assessing differences in wound colonization.

\section{Scl1 alters human-cell integrin binding to EDA/cFn}

EDA and EDB are highly included in cFn isoforms expressed within wound beds and perform important functions in wound healing (33-36). Inasmuch as, the role of EDA in wound healing is well defined, limited knowledge exist for EDB. Multiple cells have been shown to bind to EDA via integrin receptors $\alpha_{9} \beta_{1}, \alpha_{4} \beta_{1}$, and $\alpha_{4} \beta_{7}$. EDB, on the contrary, is 


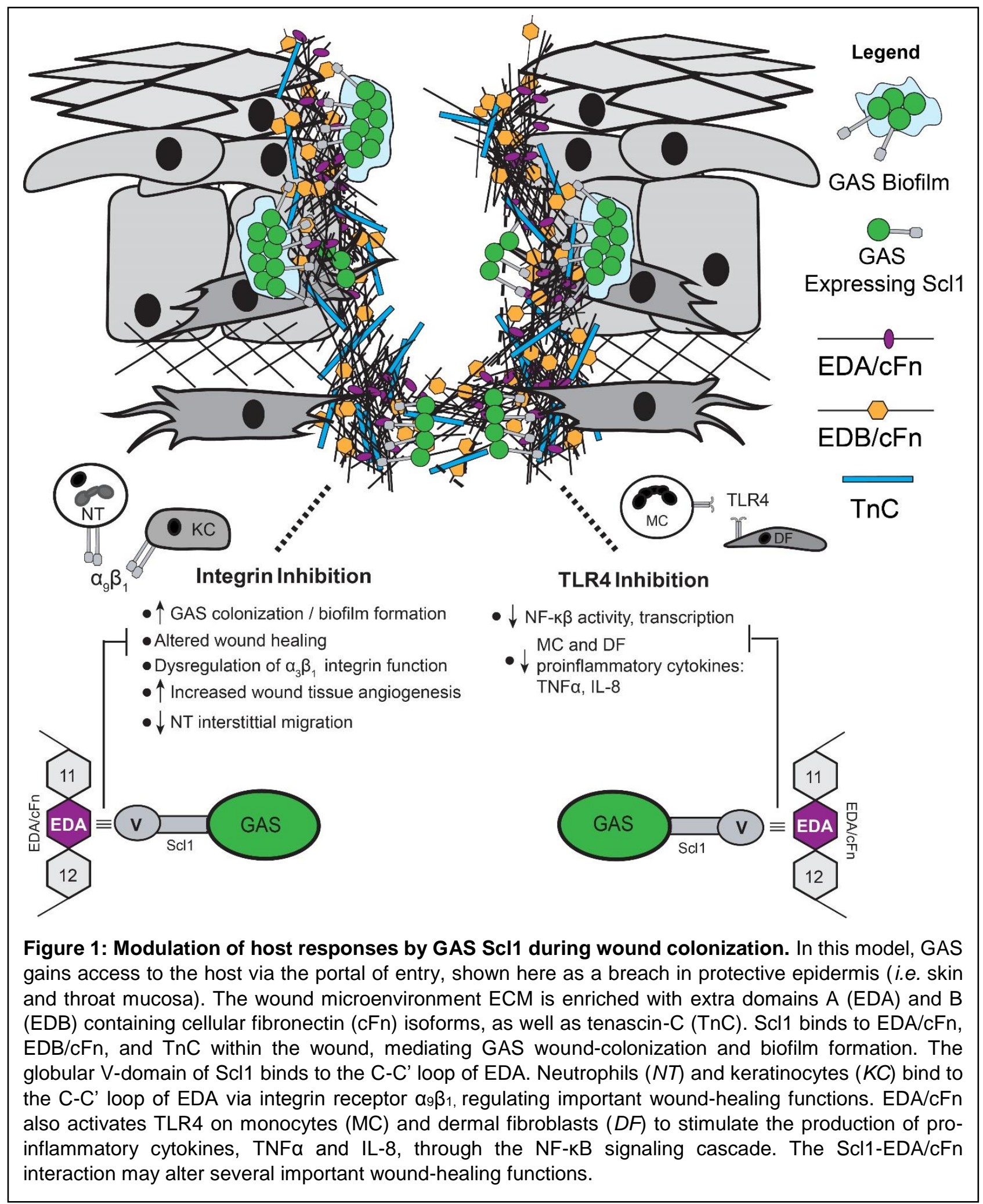

not known to interact with integrin receptors. Both $\alpha_{9} \beta_{1}$ and $\alpha_{4} \beta_{1}$ bind directly to the C-C' loop of EDA, and this binding regulates important wound-healing functions. Scl1 binding 
to EDA was also mapped to the C-C' loop (7), at or near the binding site of host cell integrin receptor $\alpha_{9} \beta_{1}$ (37). Since Scl1 and integrin $\alpha_{9} \beta_{1}$ bind to the same peptide region of the EDA segment, Scl1 may compete with $\alpha_{9} \beta_{1}$ binding to EDA, impacting functions of cells, such as keratinocytes. To this extent, our preliminary unpublished data suggests that $r S c l 1$ competes in vitro with $\alpha_{9} \beta_{1}$ binding to EDA and impedes cell attachment and spreading. By competing with the host's integrins, Scl1 binding to EDA/cFn could lead to alterations of the dynamic wound-healing processes, such as changes in angiogenesis, dysregulation of $\alpha_{3} \beta_{1}$ function, and neutrophil migration to the wound (Fig. 1).

Wound keratinocytes express $\alpha_{9} \beta_{1}$, which exerts a "trans-dominant" inhibitory effect on $\alpha_{3} \beta_{1}$ during angiogenesis in healing wounds (36). On keratinocytes, $\alpha_{3} \beta_{1}$ binds to laminin-332, and this binding mediates endothelial cell migration and angiogenesis through the secretion of growth factors $(38,39)$. Binding of $\alpha_{9} \beta_{1}$ to EDA/cFn controls $\alpha_{3} \beta_{1}$ mediated angiogenesis by modulating growth factor secretion, thereby reducing endothelial cell migration and leading to increases in endothelial cell apoptosis during the regression of growing blood vessels (36). Inhibition of $\alpha_{3} \beta_{1}$ by $\alpha_{9} \beta_{1}$ occurs through changes in Src-mediated phosphorylation of focal adhesion kinase (36). The temporal expression of EDA/cFn, during wound healing, suggests that the $\alpha_{9} \beta_{1}$-to- $\alpha_{3} \beta_{1}$ integrin cross-talk is important for controlling the level of angiogenesis. Therefore, Scl1 binding to $\mathrm{EDA} / \mathrm{cFn}$, within the $\alpha_{9} \beta_{1}$ binding site, may disrupt this inhibition, leading to dysregulated $\alpha_{3} \beta_{1}$ function (Fig. 1). This could result in: altered angiogenesis, increased keratinocyte activation and the development of hypertrophic scars (40), or aberrant tissue remodeling due to ECM production and increased MMP9 secretion $(36,41)$. Future in vitro and in vivo experiments are needed to test if Scl1 mediates such effects.

In addition to keratinocytes, the $\beta_{1}$ integrin receptors on neutrophils play important roles in inflammation and wound healing. Neutrophils are one of the first immune cells to enter into infected tissues. Neutrophils have multiple effector functions, such as the production of reactive-oxygen species, proteases, antimicrobial peptides and cytokines, as well as killing the pathogens by phagocytosis and via neutrophil extracellular traps (42). During infection, GAS causes the release of chemoattractants from the site of infection, into the blood stream that lead to the extravasation of neutrophils from the blood stream into the interstitial tissue, where they migrate through the ECM to the site of the 
infection (43). The $\alpha_{9} \beta_{1}$ expression is upregulated on activated neutrophils, and plays an essential role during neutrophil extravasation and in interstitial tissue migration $(44,45)$. The $\alpha_{9} \beta_{1}$ integrin acts in concert with $\beta_{2}$ integrins during the trans-endothelial movement of neutrophils from the bloodstream into the interstitial space (46). In vitro studies demonstrated that the $\alpha_{9} \beta_{1}$ integrin receptor significantly contributes to the passage of neutrophils through human-dermal fibroblast-derived matrices, implicating $\alpha_{9} \beta_{1}$ in the movement of neutrophils through dermal tissue (44). EDA/cFn is highly upregulated in damaged tissues (33), as well as increased in the blood in a post-surgical bacteria model in sheep (47); in vitro studies have demonstrated co-localization of fibronectin and $\alpha_{9} \beta_{1}$ in areas of tissue damage in human patients (48). It is possible that the $\alpha_{9} \beta_{1}$ integrin receptor adheres to EDA/cFn during migration through infected tissue, and Scl1 may disrupt this interaction to block neutrophil migration into damaged tissues (Fig. 1).Experiments examining neutrophil migration in the presence of Scl1, on ECM coated surfaces could address this potential impact of Scl1 on neutrophils.

\section{Perturbation of EDB/cFn and TnC function by Scl1 binding}

The role of EDB in the wound microenvironment is not well understood, as no integrinbinding sites have been identified directly within EDB. EDB/cFn isoforms are associated with angiogenesis during fetal development and within healing tissues $(49,50)$. Inclusion of EDB into cFn has been shown in vitro to contribute to head-to-tail dimerization of Fn, leading to fibril formation (51). It is possible that Scl1 binding to EDB may block important sites necessary for head-to-tail dimerization and prevent or delay fibril formation on the surface of cells. Moreover, EDB inclusion into cFn is known to expose cryptic sites that are necessary for EDB/cFn attachment to cells (52). Scl1 binding to EDB may alter the structural conformation of cFn, preventing full-exposure of those cryptic sites in EDB/cFn isoforms. Other bacterial adhesins that bind to the FnIII repeats of Fn have been shown to have a such effect on Fn structure $(53,54)$. These potential impacts of Scl1 binding to $\mathrm{EDB} / \mathrm{cFn}$ isoforms require further investigations.

Similar to affecting EDA/EDB containing cFn isoforms, TnC plays important functions in wound healing (outlined in the General Introduction). Scl1 binds within the Fnlll repeats of $\mathrm{TnC}$. The TnFnlll repeats of $\mathrm{TnC}$ have been implicated in facilitating 
fibroblast proliferation, fibronectin fibril formation, proteoglycan binding and assembly, and regulating myofibroblast contracture of wounded tissues (55-58). It is possible that Scl1 binding to TnFnlll repeats leads to alterations in fibroblast function, proper matrix assembly, fibrotic tissue development, and wound closure. It is important to note that the exact motif within the TnFnlll repeat(s) that confers these effects on host cells is not known. Specifically, the TnFn3 repeat is suggested to be bound by $\alpha_{9} \beta_{1}$ receptor on neutrophils during neutrophil migration to damaged tissue (43). Though our data does not support rScl1 binding to $\mathrm{rTnFn} 3$, it is possible that this lack of rScl1 binding to the individual $r T n F n 3$ construct is due to structural differences between the individual repeat and when it is embedded within neighboring TnFnIII repeats (e.g. Fnlll repeats 1-5 of TnC, see Chapter 2). Since the exact binding site(s) within the TnFnlll repeats for Scl1 have not been identified, these concepts require experimental testing.

\section{Scl1-EDA binding inhibits TLR4}

TLR4 is a member of the pattern recognition receptors that recognize host- and pathogenassociated molecules linked with damaged and infected tissue, and induce the release of pro-inflammatory cytokines $(59,60)$. TLR4 is expressed by immune cells such as monocytes, dendritic cells, as well as endothelial cells and fibroblasts within tissues (60). On the surface of cells, TLR4 is expressed in complex with two other surface proteins, CD14 and MD2, which facilitate proper receptor activation and ligand specificity, respectively $(61,62)$. The major agonist for TLR4 is bacterial LPS; however, several hostderived molecules have also been shown to activate TLR4, including EDA/cFn fragments (63-65).

During wound healing, one of the critical steps is tissue remodeling, fostered by matrix metalloproteinases that are secreted by both resident tissue cells and infiltrating immune cells $(66,67)$. EDA/cFn fragments, generated by this proteolysis, have been shown to activate TLR4 on dermal fibroblasts, as well as monocytes and dendritic cells $(65,67,68)$. This interaction induces the NF-KB signaling cascade, promoting proinflammatory responses, such as production of cytokines IL-8 and TNFa (65). In addition to cytokines, EDA activation of TLR4 on neutrophils and monocytes was shown to facilitate release of leukotriene $B_{4}$, a chemoattractant for leukocytes, resulting in 
increased leukocyte migration, both in vitro and in vivo (69). EDA-mediated activation of TLR4 is dependent on the TLR4-co-receptor, MD-2, and evidence suggests that activation of TLR4 is enhanced by synergy from neighboring Fn domains $(65,67,70)$.

It is currently not known, whether Scl1 binding to EDA/cFn fragments affects TLR4 function. In such a scenario, Scl1 may inhibit TLR4 activation, leading to a subsequent reduction in pro-inflammatory cytokines and a reduction of neutrophil infiltration to the site of infection (Fig. 1). This could serve as a novel Scl1-based mechanism for GAS immune evasion. To advance this project, I cloned an rFnlll11-EDA fragment, that was previously shown to stimulate TLR4 in vitro (67), into pET21a expression vector in the ClearColi $E$. coli strain, which produces a modified LPS that does not stimulate TLR4 (71). Having an LPS-free system, will facilitate our laboratory to study whether Scl1 blocks EDA/cFn mediated TLR4 activation, using an in vitro HEK-blue cell system (67). In vivo animal experiments, using WT and Scl1 mutant GAS strains could also be performed, looking for differences in tissue NF-KB activation and differences in cytokines and leukocyte recruitment in tissues.

Therefore, the Scl1 interaction between EDA and EDB/cFn isoforms, as well as $\mathrm{TnC}$, raises an exciting prospect that not only does Scl1 facilitate GAS wound colonization, but colonization of GAS via Scl1 may alter wound healing responses via competition between Scl1 and host cell receptors.

\section{Utilization of wound-associated ECM proteins to target GAS via Scl1}

Currently, GAS infections are treated either with penicillin or erythromycin in patients with a penicillin allergy (72). Alarmingly, several studies documented penicillin treatment failure, with up to $30 \%$ of pharyngitis patients showing persistence of GAS following treatment (73-76). These observations underscore the need for new alternative strategies to treat GAS infection. Considerable efforts have been undertaken by research laboratories in search of vaccine targets to prevent GAS infections and the development of a universal GAS vaccine. The immunogenic surface $M$ protein serves as the basis for most GAS vaccine candidates, as it confers M type-specific immunity (77). Historically, cross-reactive antibody concerns between $M$ protein and cardiac tissue impeded development of GAS vaccines for years; however, current methods utilize peptides 
corresponding to the $\mathrm{N}$-terminal hypervariable region, thereby avoiding cross-reactive portions $(78,79)$. A 26 -valent vaccine, which is composed of $\mathrm{N}$-terminal hypervariable region peptides derived from 26 different M-protein types, has completed stage $\mathrm{I} / \mathrm{II}$ clinical trials in the United States (80). Still, other challenges exist, such as the high number of $M$ protein types, the lack of vaccine protection from throat infections, and differences in $\mathrm{M}$ type distribution across the globe $(81,82)$. Antibodies against the Scl1 antigen were produced by humans and in experimental animals (83). In general, anti-Scl1 antibodies could be opsonic or may well block the interactions between Scl1 and EDA/cFn, thus, preventing GAS colonization. Although infection with GAS can lead to the production of anti-Scl1 antibodies, others have found that recombinant Scl-based collagen proteins are non-immunogenic (84). Variability of the amino acid sequence of the Scl1 V-domain would face challenges similar to those concerning the $M$ protein-based vaccine. Therefore, alternative targeting strategies utilizing Scl1 recognition by EDA, EDB, or TnFnlll fragments could overcome the aforementioned hurdles.

\section{EDA-derived peptides for GAS targeting}

Of the known Scl1 ligands, the C-C' loop of EDA is the best studied peptide sequence and can potentially serve as a targeting strategy for Scl1. Inasmuch as utilizing peptides derived from the C-C' loop of EDA is a rational premise for treating GAS infections, there are significant challenges that need to be overcome. First, the binding affinities between C-C'-loop circular peptide and two different recombinant Scl1 variants, originating from M1- and M3-type GAS, were in a range of 40-60 $\mu \mathrm{M} K_{D}$, as estimated through surface plasmon resonance and tryptophan fluorescence binding assays $((24,85)$ appendix 2 and 3). This binding is relatively weak, especially when compared with the binding affinity of an antibody, typically in the $\mathrm{nM}$ range, and mutagenesis approach may improve the binding affinity. Second, off-target binding effects of the C-C' loop peptide directly to host cell integrin receptor $\alpha_{9} \beta_{1}$ could lead to dysregulation of the dynamic wound-healing processes, such as dysregulation in tissue angiogenesis (Fig. 1). Alternatively, peptides derived from EDB could be used in targeting Scl1 on GAS. Third, although all strains carry the scl1 gene, a small subset of strains do not express Scl1, such as M3-type GAS (24). In these strains, however, Scl2 protein would serve as an alternative target. Fourth, the 
C-C' loop-peptide could increase bacterial spread via microcolony dispersion. Nevertheless, sessile GAS cells will be susceptible to drugs or C-C' loop-derived peptide conjugated to an antimicrobial peptide, like LL-37 $(86,87)$. A similar strategy, utilizing peptides derived from EDB and/or TnFnlll repeats to target Scl1 would be feasible.

\section{Anti-Scl1 Fnlll-based monobodies for GAS targeting}

Alternatively to the utilization of EDA-derived peptides, the whole EDA or a modified FnIII repeat, known as a monobody (88), could be used in targeting Scl1. Monobodies are protein constructs that utilize FnIll scaffolds to achieve specific protein binding, as they share structural homology to the antigen recognition region of antibodies (Fig. 2), and serve as an alternative to antibodies $(89,90)$. While commonly used in medicine, monoclonal antibodies are large in size $(\sim 150 \mathrm{kDa})$ limiting their tissue distribution; Moreover, monoclonal antibodies are complex to produce (91). Monobodies, however, are much smaller ( 10-20 kDa) enabling effective tissue distribution, are less complex to produce, and can exhibit $\mathrm{nM}$ and $\mathrm{pM}$ affinities to their designed targets, similar to monoclonal antibodies (91-93). The surface exposed loops within the monobody (Fig. 2) serve as the ligand-recognition site and can be engineered to bind a multitude of epitopes (90). Monobodies serve as diagnostic and therapeutic tools, often via the conjugation of markers or drugs (94). Originally, monobodies were developed using the Fnll repeat number 10 from fibronectin (95), however, monobodies derived from other fibronectin Fnlll repeats of both Fn and $\mathrm{TnC}$ are currently undergoing clinical trials (94). The EDB of cFn could serve as a monobody to target GAS via Scl1, as it offers the benefit of reduced off-target binding, compared to EDA, which has the potential to bind to cells expressing integrin receptors $\alpha_{4} \beta_{1}, \alpha_{4} \beta_{7}, \alpha_{9} \beta_{1}$, as well as stimulate TLR4. Conversely, EDA may serve as the preferential monobody, as the Scl1-binding site and potential binding affinities have been delineated $(7,24)$. The EDA or EDB-derived monobody can be conjugated directly to antimicrobial peptides or anti-microbials to treat GAS infection, localizing antibiotics to the site of GAS infection, reducing impacts on the microbiome. 

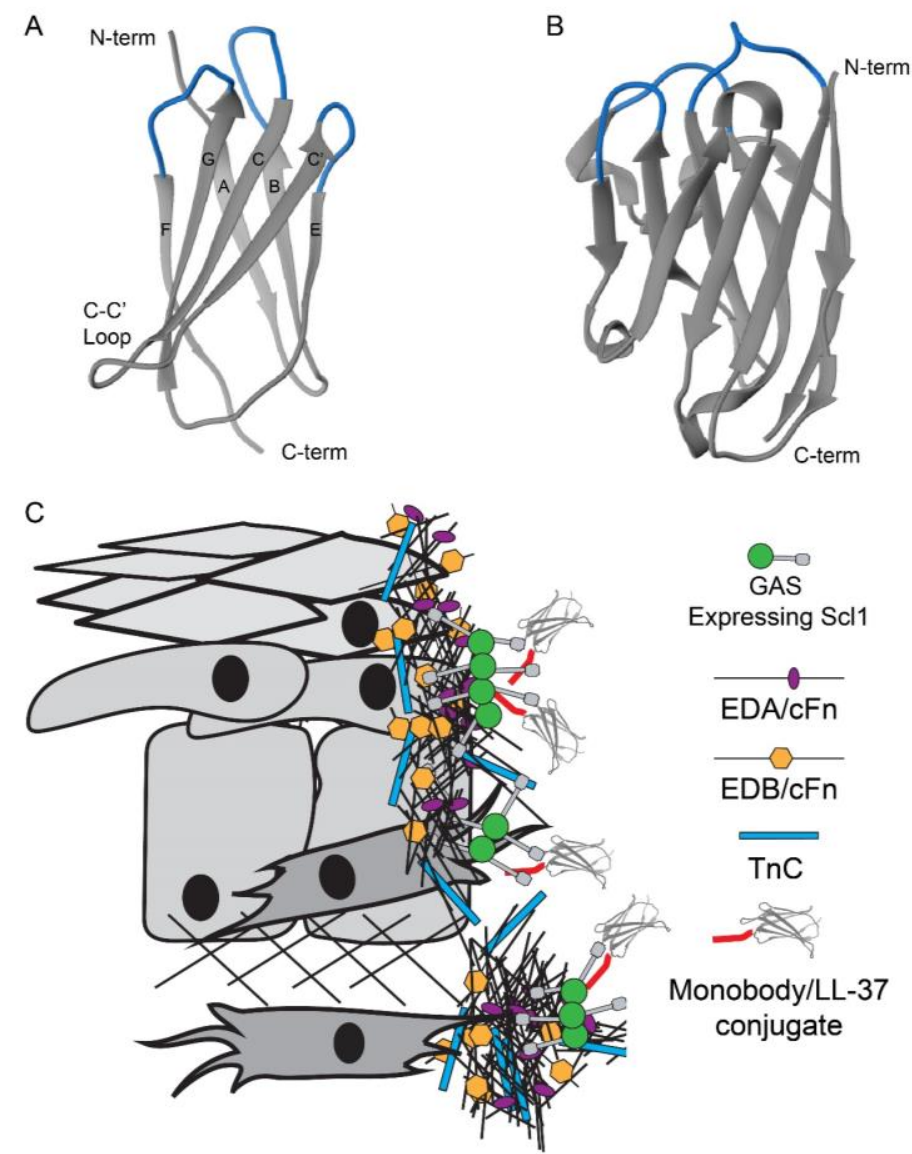

Figure 2: Monobodies to target GAS via Scl1-. A) Cartoon representation of the fibronectin type III (FnIII) repeat, extra domain B (EDB), from cellular fibronectin (PDB accession code: 2GEE); showing the fibronectin-type III repeat $\beta$-sandwich structure, with each $\beta$-strands marked $\left(A, B, C, C^{\prime}, D, E, F\right)$. Loops of EDB that share similar orientation to the antigenbinding domain of single-chain antibodies are highlighted in blue $\mathbf{B})$ Cartoon representation of the antigen-binding domain of a camelid heavy-chain antibody $\left(\mathrm{V}_{\mathrm{H}} \mathrm{H} ; \mathrm{PDB}\right.$ accession code: 1ZVY); showing that both EDB and the antigen-binding domain of antibodies consist of a Fnlll repeat structure. The antigen-recognition sites are highlighted in blue. C) Schematic, depicting the rationale behind monobody targeting of group A Streptococcus (GAS) via the streptococcal collagen-like 1 (Scl1) surface adhesin. Due to shared structures, Fnlll repeats can be engineered to as antibody mimics. Scl1 binds to isoforms of cellular fibronectin that contain EDA, EDB, as well as tenascin-C (TnC), which are associated-with the wound microenvironment. During host wound colonization, EDB-derived monobodies that are conjugated to anti-microbial peptides (e.g. LL-37, in red) can be administered to wound to target and kill GAS.

\section{Implications of Scl1 in cancer targeting}

Isoforms of $\mathrm{CFn}$ that contain EDA and/or EDB, as well as TnC, are expressed in different types of cancer, such as prostate cancer, colorectal cancer, head and neck squamous cell carcinoma, cervical cancer, and lung cancer (96-101). Expression and deposition of EDA/EDB cFn isoforms, and TnC, is predominantly done by the surrounding stromal cells, 
primarily by cancer-associated fibroblasts (CAFs)/myofibroblasts, but also by endothelial cells during neovascularization of growing tumors $(102,103)$. Some cancer cells will directly produce EDA/EDB cFn, but $\mathrm{TnC}$ has been shown to be directly deposited on the invasive edge of malignant cancer cells and is found to be deposited in newly localized metastatic cancers $(104,105)$. Current research supports a model where cancer progression is driven by the surrounding cancer microenvironment, rather than intrinsic cancer cell factors (106), as expression of these ECM proteins by CAFs is linked to several important pathological mechanisms of cancer, such as the epithelial to mesenchymal transition, cell proliferation, and metastasis $(107,108)$. Epidemiological studies have found that patients with elevated deposition of EDA/EDB cFn isoforms or $\mathrm{TnC}$ within their tumors have lower survival rates compared to patients with lower levels of EDA and EDB cFn isoforms and TnC (109,110). Thus, these ECM substrates are unique targets for the delivery of diagnostics and therapeutics to the tumor sites. Several research groups and companies across the world have developed monoclonal antibodydrug conjugates, peptides and aptamers to target EDA/EDB cFn isoforms and to $\mathrm{TnC}$ for such purposes $(111,112)$. We have shown that Scl1 specifically targets EDA/EDB, and the Fnlll repeats of $\mathrm{TnC}$ within the matrices deposited by CAFs (Fig. 3). Thus, Scl1 offers similar potential as a biomolecule for the selective recognition and targeting of GAS or Scl1-functionalized nanomaterials to cancerous tissue.

\section{GAS infection in cancer immunotherapy}

GAS has a historical connection with cancers, particularly sarcomas, as it was noted since the early 1800s that patients who develop erysipelas, commonly caused by GAS, demonstrated shrinkage of their tumors (113). Currently, there is interest in using microbes in the treatment of cancer. Microbes offer advantages to traditional chemotherapies, as they can be engineered to favorably colonize the tumor microenvironment and lead to the death of cancer cells either directly through toxin production or indirectly via immune stimulation (113). While there is renewed interest in the utilization of GAS as a cancer-therapy, showing promise in the treatment of pancreatic cancer in a murine model $(114,115)$, there are still fears of the development of 

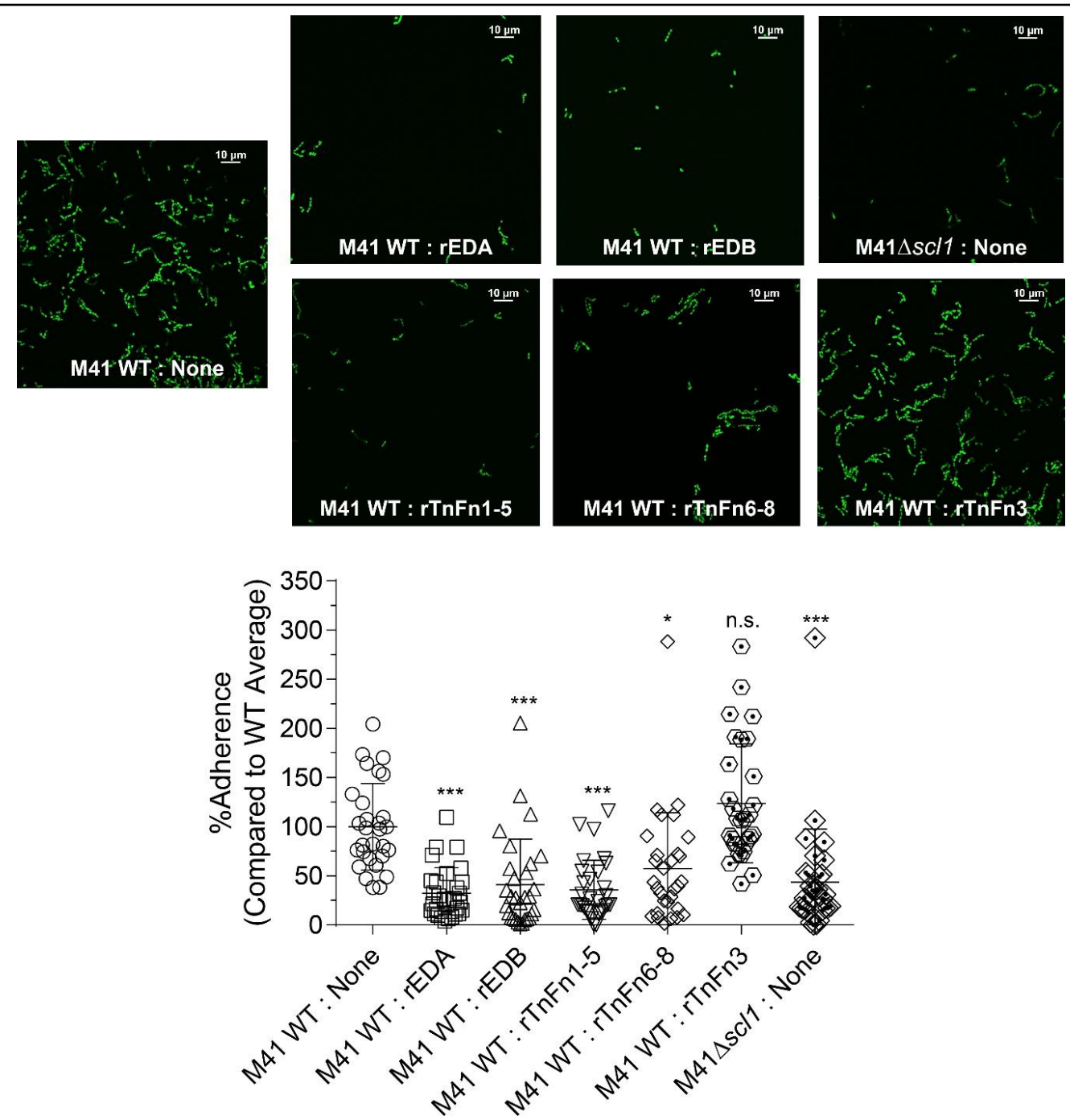

GAS Strain

Figure 3. Specificity of Scl1-mediated GAS attachment to cancer-associated fibroblast matrices. Wild type (WT) M41 was pre-incubated with recombinant ECM ligands prior to attachment to CAF-derived ECM. GFP-WT M41 GAS were pre-incubated with recombinantly expressed extra domain $A$, extra domain $B$, and fibronectin type III repeats of tenascin- $C$, for 30 minutes prior to inoculation onto CAF-derived ECM coatings. GAS were allowed to attach for 1 hour and then subsequently imaged using fluorescent confocal microscope with 100x objective. Images were taken in 10 random fields over three experiments. Top, representative images Bottom, quantification of GAS attachment with WT binding set as $100 \%$; bacteria were counted in 30 fields, and the average from all 30 -fields was calculated. Percentage based on the average number of counted bacteria for the parental WT strain. Statistical significance was calculated using a one-way ANOVA from three independent experiments, each performed in duplicate wells $(N=3 \pm S D) ;{ }^{* *} P \leq 0.01,{ }^{* *} P \leq 0.001$. Statistical analysis evaluates the difference in adherence between WT GAS and WT GAS pre-incubated with ECM. Each symbol represents one image.

autoimmune sequalae, such as rheumatic fever, in patients treated with GAS. Other candidate bacteria do not share this concern, such as a Salmonella typhimurium and Clostridium novyi, and treatments are under development or in clinical trials to evaluate 
their efficacy $(116,117)$. Yet, significant challenges remain in the utilization of these candidate bacteria in the treatment of cancer, such as the improved bacterial colonization and retention within the cancer tissues and the development of multi-tissue specific bacteria that can target metastatic cancers (113).

\section{rScl1-functionalized nanoparticles for cancer diagnostics and treatment}

Another approach is the use of $\mathrm{rScl} 1$ proteins as targeting biomolecules on the surface of nanoparticles for targeted drug delivery to cancer tissues. Nanomaterials offer unique advantages, due to their size, durability, surface area, and composition, relative to

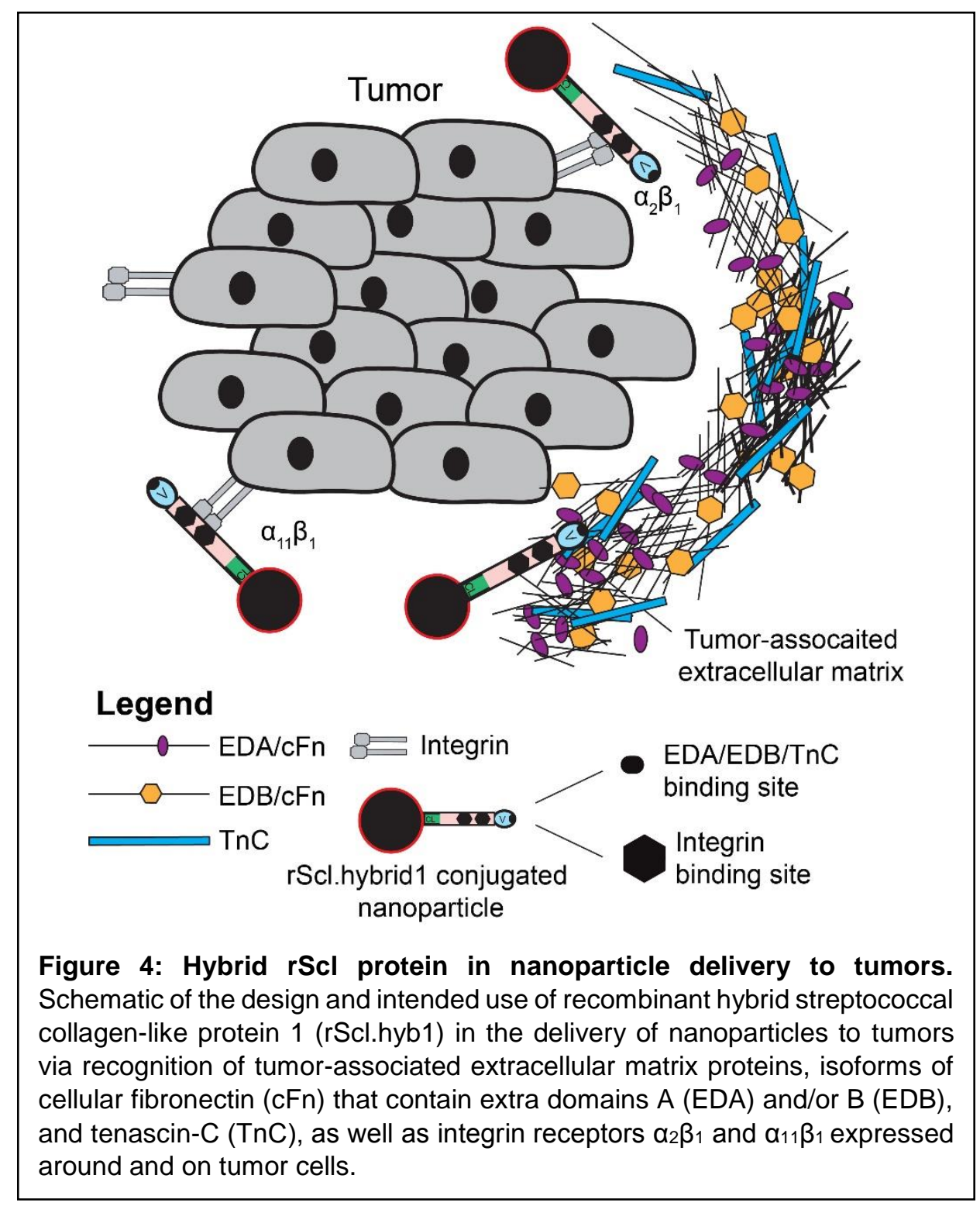


bacteria. Moreover, nanoparticles do not pose the same off-target infection risks as bacterial-based cancer immunotherapies. Nanoparticles can be loaded with drugs (e.g. doxorubicin) or biologicals (e.g. siRNA) for delivery to cancer cells $(118,119)$. However, one of the major challenges to nanoparticle cancer therapy is the lack of localization and retention of particles within the cancer microenvironment. This is due in part to the lack of targets unique to the cancer microenvironment, not present healthy tissue (106). Targeting EDA/EDB cFn isoforms and TnC, using rScl-based proteins may solve this problem. Other groups have developed aptamers and antibodies that target these ECM proteins with the same goal in mind, some of which have been conjugated to nanomaterials $(111,119,120)$. Scl-derived proteins may have superior features, like 1) Scl1 proteins bind multiple cancer-associated ligands, thereby reducing the number of different biomolecules needed to be conjugated to a nanomaterial, and 2) the potential to target integrin receptors on tumor cells through incorporation of the $\alpha_{2} \beta_{1}$ and $\alpha_{11} \beta_{1}$ recognition motif (e.g. GLPGER) into the CL-domain of Scl proteins (121). As a part of my IGERT research, I helped engineer a hybrid rScl protein (rScl.hybrid1; Fig. 4) that possess both the EDA/EDB and TnC binding site in the V-domain, as well as integrin recognition sequences within the $\mathrm{CL}$-domain. This hybrid protein $\mathrm{rScl}$.hybrid1 offers the potential to target $\mathrm{TnC}$, as well as EDA/cFn and EDB/cFn isoforms, highly expressed within and around tumor/cancerous tissues, as well as promote internalization via integrin receptors $\alpha_{2} \beta_{1}$ and $\alpha_{11} \beta_{1}$ (Fig. 4)(122,123).

\section{Overall Conclusions}

My work identified important structural determinants that facilitate Scl1 ligand-binding, highlighting a binding mechanism that is unique relative to the other Fn-binding proteins of GAS. In addition, I discovered that Scl1 binds to the FnIII repeats, EDB of cFn, as well as to the Fnlll repeats within $\mathrm{TnC}$, increasing the number of known targets for Scl1 that are associated with the wound microenvironment. My work adds new evidence in support that $\mathrm{Scl} 1$ is a wound-adapted adhesin for GAS.

As GAS is a human-obligate pathogen, it has co-evolved with us over time, evident through the plethora of virulence factors that GAS employs during pathogenesis to facilitate host colonization (124). Scl1 is a major GAS adhesin, and is under the regulation 
of one of the major transcriptional regulators, Mga, which controls the expression of several other key GAS virulence factors (23,125-129). Scl1 is expressed by almost every $M$ type studied (23), and as a surface protein, Scl1 is under selective pressure which results in sequence variation within the Scl1-V domain across different M-types of GAS (23). Yet, despite this sequence variation, Scl1 proteins from across different M-types maintain the capacity to bind to EDA, EDB, and TnC, underscoring both the evolution of this adhesin for host adaptation and the importance of these ligands for GAS. The first environment GAS will typically encounter within our bodies is wounded tissue. This selective binding of Scl1 to Fnlll repeats that are highly associated with wounded tissue, in addition to the conservation of this interactions across phylogenetically-diverse $M$ types, serves as strong evidence that Scl1 has adapted to the wound microenvironment, contributing to GAS wound colonization.

The work presented here lays the foundation for several future directions to take research pertaining to Scl1, having implications in the development of strategies to target GAS using Fnlll monobody, or the use of Scl1 proteins in targeting cancers for drug delivery. Ultimately, the strongest implications of this research are in the roles the Scl1$\mathrm{EDA} / \mathrm{EDB} / \mathrm{TnC}$ interactions play in GAS pathogenesis, altering host wound healing and in mediating GAS wound colonization.

\section{REFERENCES}

1. Yamaguchi, M., Terao, Y., and Kawabata, S. (2013) Pleiotropic virulence factor - Streptococcus pyogenes fibronectin-binding proteins. Cell. Microbiol. 15, 503-511

2. Schwarz-Linek, U., Werner, J. M., Pickford, A. R., Gurusiddappa, S., Kim, J. H., Pilka, E. S., Briggs, J. A., Gough, T. S., Höök, M., Campbell, I. D., and Potts, J. R. (2003) Pathogenic bacteria attach to human fibronectin through a tandem beta-zipper. Nature 423, 177-181

3. McNitt, D. H., Choi, S. J., Keene, D. R., Van De Water, L., Squeglia, F., Berisio, R., and Lukomski, S. (2018) Surface-exposed loops and an acidic patch in the Scl1 protein of group A Streptococcus enable Scl1 binding to wound-associated fibronectin. J. Biol. Chem. 293, 77967810

4. Back, C. R., Sztukowska, M. N., Till, M., Lamont, R. J., Jenkinson, H. F., Nobbs, A. H., and Race, P. R. (2017) The Streptococcus gordonii adhesin CshA protein binds host fibronectin via a catchclamp mechanism. J Biol Chem 292, 1538-1549 
5. Rego, S., Heal, T. J., Pidwill, G. R., Till, M., Robson, A., Lamont, R. J., Sessions, R. B., Jenkinson, H. F., Race, P. R., and Nobbs, A. H. (2016) Structural and functional analysis of cell wall-anchored polypeptide adhesin BspA in Streptococcus agalactiae. J. Biol. Chem. 291, 1598516000

6. Caswell, C. C., Oliver-Kozup, H., Han, R., Lukomska, E., and Lukomski, S. (2010) Scl1, the multifunctional adhesin of group A Streptococcus, selectively binds cellular fibronectin and laminin, and mediates pathogen internalization by human cells. FEMS Microbiol. Lett. 303, 61-68

7. Oliver-Kozup, H., Martin, K. H., Schwegler-Berry, D., Green, B. J., Betts, C., Shinde, A. V., Van De Water, L., and Lukomski, S. (2013) The group A streptococcal collagen-like protein-1, Scl1, mediates biofilm formation by targeting the extra domain A-containing variant of cellular fibronectin expressed in wounded tissue. Mol. Microbiol. 87, 672-689

8. Hemperly, J. J., Murray, B. A., Edelman, G. M., and Cunningham, B. A. (1986) Sequence of a cDNA clone encoding the polysialic acid-rich and cytoplasmic domains of the neural cell adhesion molecule N-CAM. Proc. Natl. Acad. Sci. U. S. A. 83, 3037-3041

9. Frick, I. M., Crossin, K. L., Edelman, G. M., and Björck, L. (1995) Protein H-a bacterial surface protein with affinity for both immunoglobulin and fibronectin type III domains. EMBO J. 14, 16741679

10. Jaffe, J., Natanson-Yaron, S., Caparon, M. G., and Hanski, E. (1996) Protein F2, a novel fibronectin-binding protein from Streptococcus pyogenes, possesses two domains. Mol. Microbiol. 21, 373-384

11. Kreikemeyer, B., Oehmcke, S., Nakata, M., Hoffrogge, R., and Podbielski, A. (2004) Streptococcus pyogenes Fibronectin-binding Protein F2: Expression profile, binding characteristics, and impact on eukaryotic cell interactions. J. Biol. Chem. 279, 15850-15859

12. Henderson, B., Nair, S., Pallas, J., and Williams, M. A. (2011) Fibronectin: a multidomain host adhesin targeted by bacterial fibronectin-binding proteins. FEMS Microbiol. Rev. 35, 147-200

13. Cameron, C. E., Brown, E. L., Kuroiwa, J. M. Y., Schnapp, L. M., and Brouwer, N. L. (2004) Treponema pallidum fibronectin-binding proteins. J. Bacteriol. 186, 7019-7022

14. Schulze-Koops, H., Burkhardt, H., Heesemann, J., Kirsch, T., Swoboda, B., Bull, C., Goodman, S., and Emmrich, F. (1993) Outer membrane protein YadA of enteropathogenic yersiniae mediates specific binding to cellular but not plasma fibronectin. Infect. Immun. 61, 2513-2519

15. Heise, T., and Dersch, P. (2006) Identification of a domain in Yersinia virulence factor YadA that is crucial for extracellular matrix-specific cell adhesion and uptake. Proc. Natl. Acad. Sci. U. S. A. 103, 3375-3380

16. Sakai, T., Johnson, K. J., Murozono, M., Sakai, K., Magnuson, M. A., Wieloch, T., Cronberg, T., Isshiki, A., Erickson, H. P., and Fassler, R. (2001) Plasma fibronectin supports neuronal survival and reduces brain injury following transient focal cerebral ischemia but is not essential for skinwound healing and hemostasis. Nat. Med. 7, 324-330 
17. Schenk, S., Liénard, D., Gérain, J., Baumgartner, M., Lejeune, F. J., Chiquet-Ehrismann, R., and Rüegg, C. (1995) Rapid increase in plasma tenascin-C concentration after isolated limb perfusion with high-dose tumor necrosis factor (TNF), interferon gamma (IFNY) and melphalan for regionally advanced tumors. Int. J. Cancer 63, 665-672

18. Midwood, K. S., Hussenet, T., Langlois, B., and Orend, G. (2011) Advances in tenascin-C biology. Cell. Mol. Life Sci. 68, 3175

19. Barnes, J. L., Torres, E. S., Mitchell, R. J., and Peters, J. H. (1995) Expression of alternatively spliced fibronectin variants during remodeling in proliferative glomerulonephritis. Am. J. Pathol. 147, 1361-1371

20. Gratchev, A., Guillot, P., Hakiy, N., Politz, O., Orfanos, C. E., Schledzewski, K., and Goerdt, S. (2001) Alternatively activated macrophages differentially express fibronectin and its splice variants and the extracellular matrix protein BIG-H3. Scand. J. Immunol. 53, 386-392

21. Musser, J. M., Hauser, A. R., Kim, M. H., Schlievert, P. M., Nelson, K., and Selander, R. K. (1991) Streptococcus pyogenes causing toxic-shock-like syndrome and other invasive diseases: clonal diversity and pyrogenic exotoxin expression. Proc. Natl. Acad. Sci. U. S. A. 88, 2668-2672

22. Lamagni, T., Darenberg, J., Luca-Harari, B., Siljander, T., Efstratiou, A., Henriques-Normark, B., Vuopio-Varkila, J., Bouvet, A., Creti, R., Ekelund, K., Koliou, M., Reinert, R., Stathi, A., Strakova, L., Ungureanu, V., Schalen, C., , and Jasir, A. (2008) The epidemiology of severe Streptococcus pyogenes disease in Europe. J. Clin. Microbiol.

23. Lukomski, S., Nakashima, K., Abdi, I., Cipriano, V. J., Ireland, R. M., Reid, S. D., Adams, G. G., and Musser, J. M. (2000) Identification and characterization of the $\mathrm{scl}$ gene encoding a group $A$ Streptococcus extracellular protein virulence factor with similarity to human collagen. Infect. Immun. 68, 6542-6553

24. Bachert, B. A., Choi, S. J., LaSala, P. R., Harper, T. I., McNitt, D. H., Boehm, D. T., Caswell, C. C., Ciborowski, P., Keene, D. R., Flores, A. R., Musser, J. M., Squeglia, F., Marasco, D., Berisio, R., and Lukomski, S. (2016) Unique footprint in the sc/1.3 locus affects adhesion and biofilm formation of the invasive M3-type group A Streptococcus. Front Cell Infect Microbio/ 6, 90

25. Flores, A. R., Jewell, B. E., Versalovic, E. M., Olsen, R. J., Bachert, B. A., Lukomski, S., and Musser, J. M. (2015) Natural variant of collagen-like protein A in serotype M3 Group A Streptococcus increases adherence and decreases invasive potential. Infect. Immun. 83, 11221129

26. Akiyama, H., Morizane, S., Yamasaki, O., Oono, T., and Iwatsuki, K. (2003) Assessment of Streptococcus pyogenes microcolony formation in infected skin by confocal laser scanning microscopy. J. Dermatol. Sci. 32, 193-199

27. Siemens, N., Chakrakodi, B., Shambat, S. M., Morgan, M., Bergsten, H., Hyldegaard, O., Skrede, S., Arnell, P., Madsen, M. B., Johansson, L., Juarez, J., Bosnjak, L., Mörgelin, M., Svensson, M., 
and Norrby-Teglund, A. (2016) Biofilm in group A streptococcal necrotizing soft tissue infections. JCl Insight 1

28. Sumby, P., Barbian, K., Gardner, D., Whitney, A., Welty, D., Long, R., Bailey, J., Parnell, M., Hoe, N., Adams, G., Deleo, F., and Musser, J. (2005) Extracellular deoxyribonuclease made by group A Streptococcus assists pathogenesis by enhancing evasion of the innate immune response., -

29. Ogawa, T., Terao, Y., Okuni, H., Ninomiya, K., Sakata, H., Ikebe, K., Maeda, Y., and Kawabata, S. (2011) Biofilm formation or internalization into epithelial cells enable Streptococcus pyogenes to evade antibiotic eradication in patients with pharyngitis. Microb. Pathog. 51, 58-68

30. Tan, M. H., Sun, Z., Opitz, S. L., Schmidt, T. E., Peters, J. H., and George, E. L. (2004) Deletion of the alternatively spliced fibronectin EIIIA domain in mice reduces atherosclerosis. Blood 104, 11-18

31. Fukuda, T., Yoshida, N., Kataoka, Y., Manabe, R.-i., Mizuno-Horikawa, Y., Sato, M., Kuriyama, K., Yasui, N., and Sekiguchi, K. (2002) Mice lacking the EDB segment of fibronectin develop normally but exhibit reduced cell growth and fibronectin matrix assembly in vitro. Cancer Res. 62, 5603-5610

32. Saga, Y., Yagi, T., Ikawa, Y., Sakakura, T., and Aizawa, S. (1992) Mice develop normally without tenascin. Genes Dev. 6, 1821-1831

33. Ffrench-Constant, C., Van De Water, L., Dvorak, H. F., and Hynes, R. O. (1989) Reappearance of an embryonic pattern of fibronectin splicing during wound healing in the adult rat. J. Cell Biol. 109, 903-914

34. Singh, P., Reimer, C. L., Peters, J. H., Stepp, M. A., Hynes, R. O., and Van De Water, L. (2004) The spatial and temporal expression patterns of integrin $\alpha 9 \beta 1$ and one of its ligands, the EIIIA segment of fibronectin, in cutaneous wound healing. J. Invest. Dermatol. 123, 1176-1181

35. Muro, A. F., Chauhan, A. K., Gajovic, S., laconcig, A., Porro, F., Stanta, G., and Baralle, F. E. (2003) Regulated splicing of the fibronectin EDA exon is essential for proper skin wound healing and normal lifespan. J. Cell Biol. 162, 149-160

36. Longmate, W. M., Lyons, S. P., Chittur, S. V., Pumiglia, K. M., Van De Water, L., and DiPersio, C. M. (2017) Suppression of integrin $\alpha 3 \beta 1$ by $\alpha 9 \beta 1$ in the epidermis controls the paracrine resolution of wound angiogenesis. J. Cell Biol. 216, 1473-1488

37. Liao, Y. F., Gotwals, P. J., Koteliansky, V. E., Sheppard, D., and Van De Water, L. (2002) The EIIIA segment of fibronectin is a ligand for integrins $\alpha 9 \beta 1$ and $\alpha 4 \beta 1$ providing a novel mechanism for regulating cell adhesion by alternative splicing. J Biol Chem 277, 14467-14474

38. Mitchell, K., Szekeres, C., Milano, V., Svenson, K. B., Nilsen-Hamilton, M., Kreidberg, J. A., and DiPersio, C. M. (2009) a3 $\beta 1$ integrin in epidermis promotes wound angiogenesis and keratinocyte-to-endothelial-cell crosstalk through the induction of MRP3. J. Cell Sci. 122, 17781787 
39. Longmate, W. M., Monichan, R., Chu, M.-L., Tsuda, T., Mahoney, M. G., and DiPersio, C. M. (2014) Reduced fibulin-2 contributes to loss of basement membrane integrity and skin blistering in mice lacking integrin $\alpha 3 \beta 1$ in the epidermis. J. Invest. Dermatol. 134, 1609-1617

40. Machesney, M., Tidman, N., Waseem, A., Kirby, L., and Leigh, I. (1998) Activated keratinocytes in the epidermis of hypertrophic scars. Am. J. Pathol. 152, 1133-1141

41. Longmate, W. M., Lyons, S. P., DeFreest, L., Van De Water, L., and DiPersio, C. M. (2018) Opposing Roles of Epidermal Integrins $\alpha 3 \beta 1$ and $\alpha 9 \beta 1$ in Regulation of mTLD/BMP-1-Mediated Laminin-y2 Processing during Wound Healing. J. Invest. Dermatol. 138, 444-451

42. Mayadas, T. N., Cullere, X., and Lowell, C. A. (2014) The multifaceted functions of neutrophils. Annu. Rev. Pathol. 9, 181-218

43. Lindbom, L., and Werr, J. (2002) Integrin-dependent neutrophil migration in extravascular tissue. Semin. Immunol. 14, 115-121

44. Shang, T., Yednock, T., and Issekutz, A. C. (1999) a9ß1 Integrin is expressed on human neutrophils and contributes to neutrophil migration through human lung and synovial fibroblast barriers. J. Leukoc. Biol. 66, 809-816

45. Taooka, Y., Chen, J., Yednock, T., and Sheppard, D. (1999) The integrin alpha9beta1 mediates adhesion to activated endothelial cells and transendothelial neutrophil migration through interaction with vascular cell adhesion molecule-1. J. Cell Biol. 145, 413-420

46. Mambole, A., Bigot, S., Baruch, D., Lesavre, P., and Halbwachs-Mecarelli, L. (2010) Human neutrophil integrin $\alpha 9 \beta 1$ : up-regulation by cell activation and synergy with $\beta 2$ integrins during adhesion to endothelium under flow. J. Leukoc. Biol. 88, 321-327

47. Rizk, T. A., Rebres, R. A., Vincent, P. A., Charash, W. E., McKeown-Longo, P. J., Lewis, E. P., Brien, T. P., Minnear, F. L., Fortune, J. B., and Saba, T. M. (1994) Delayed elevation of ED1cellular fibronectin in plasma following postsurgical bacteremia. Am J Physiol 266, L689-L697

48. Weinacker, A., Ferrando, R., Elliott, M., Hogg, J., Balmes, J., and Sheppard, D. (1995) Distribution of integrins $\alpha \vee \beta 6$ and $\alpha 9 \beta 1$ and their known ligands, fibronectin and tenascin, in human airways. Am. J. Respir. Cell Mol. Biol. 12, 547-556

49. Castellani, P., Viale, G., Dorcaratto, A., Nicolo, G., Kaczmarek, J., Querze, G., and Zardi, L. (1994) The fibronectin isoform containing the ED-B oncofetal domain: A marker of angiogenesis. Int. J. Cancer 59, 612-618

50. Birchler, M., Neri, G., Tarli, L., Halin, C., Viti, F., and Neri, D. (1999) Infrared photodetection for the in vivo localisation of phage-derived antibodies directed against angiogenic markers. $J$. Immunol. Methods 231, 239-248

51. Schiefner, A., Gebauer, M., and Skerra, A. (2012) Extra-domain B in oncofetal fibronectin structurally promotes fibrillar head-to-tail dimerization of extracellular matrix protein. J. Biol. Chem. 287, 17578-17588 
52. Chen, S., Chakrabarti, R., Keats, E. C., Chen, M., Chakrabarti, S., and Khan, Z. A. (2012) Regulation of Vascular Endothelial Growth Factor Expression by Extra Domain B Segment of Fibronectin in Endothelial CellsVEGF Expression by Fibronectin. Invest. Ophthalmol. Vis. Sci. 53, 8333-8343

53. Liang, X., Garcia, B. L., Visai, L., Prabhakaran, S., Meenan, N. A. G., Potts, J. R., Humphries, M. J., and Höök, M. (2016) Allosteric Regulation of Fibronectin/a5ß1 Interaction by FibronectinBinding MSCRAMMs. PLoS One 11, e0159118

54. Prabhakaran, S., Liang, X., Skare, J. T., Potts, J. R., and Höök, M. (2009) A Novel Fibronectin Binding Motif in MSCRAMMs Targets F3 Modules. PLoS One 4, e5412

55. End, P., Panayotou, G., Entwistle, A., Waterfield, M. D., and Chiquet, M. (1992) Tenascin: a modulator of cell growth. Eur. J. Biochem. 209, 1041-1051

56. Kusubata, M., Hirota, A., Ebihara, T., Kuwaba, K., Matsubara, Y., Sasaki, T., Kusakabe, M., Tsukada, T., Irie, S., and Koyama, Y. (1999) Spatiotemporal changes of fibronectin, tenascin-C, fibulin-1, and fibulin-2 in the skin during the development of chronic contact dermatitis. J. Invest. Dermatol. 113, 906-912

57. Day, J. M., Olin, A. I., Murdoch, A. D., Canfield, A., Sasaki, T., Timpl, R., Hardingham, T. E., and Aspberg, A. (2004) Alternative splicing in the aggrecan G3 domain influences binding interactions with tenascin-C and other extracellular matrix proteins. J Biol Chem 279, 12511-12518

58. Tamaoki, M., Imanaka-Yoshida, K., Yokoyama, K., Nishioka, T., Inada, H., Hiroe, M., Sakakura, T., and Yoshida, T. (2005) Tenascin-C regulates recruitment of myofibroblasts during tissue repair after myocardial injury. Am. J. Pathol. 167, 71-80

59. Medzhitov, R. (2001) Toll-like receptors and innate immunity. Nat. Rev. Immunol. 1, 135-145

60. Vaure, C., and Liu, Y. (2014) A Comparative Review of Toll-Like Receptor 4 Expression and Functionality in Different Animal Species. Front. Immunol. 5

61. Zanoni, I., Ostuni, R., Marek, Lorri R., Barresi, S., Barbalat, R., Barton, Gregory M., Granucci, F., and Kagan, Jonathan C. (2011) CD14 Controls the LPS-Induced Endocytosis of Toll-like Receptor 4. Cell 147, 868-880

62. Vašl, J., Oblak, A., Gioannini, T. L., Weiss, J. P., and Jerala, R. (2009) Novel Roles of Lysines 122, 125, and 58 in Functional Differences between Human and Murine MD-2. The Journal of Immunology 183, 5138-5145

63. Choi, S.-H., Harkewicz, R., Lee Jee, H., Boullier, A., Almazan, F., Li Andrew, C., Witztum Joseph, L., Bae Yun, S., and Miller Yury, I. (2009) Lipoprotein Accumulation in Macrophages via Toll-Like Receptor-4-Dependent Fluid Phase Uptake. Circ. Res. 104, 1355-1363

64. Kim, F., Pham, M., Luttrell, I., Bannerman Douglas, D., Tupper, J., Thaler, J., Hawn Thomas, R., Raines Elaine, W., and Schwartz Michael, W. (2007) Toll-Like Receptor-4 Mediates Vascular Inflammation and Insulin Resistance in Diet-Induced Obesity. Circ. Res. 100, 1589-1596 
65. Okamura, Y., Watari, M., Jerud, E. S., Young, D. W., Ishizaka, S. T., Rose, J., Chow, J. C., and Strauss, J. F., 3rd. (2001) The extra domain A of fibronectin activates Toll-like receptor 4. J Biol Chem 276, 10229-10233

66. Xue, M., and Jackson, C. J. (2015) Extracellular matrix reorganization during wound healing and its impact on abnormal scarring. Adv. Wound Care 4, 119-136

67. Julier, Z., Martino, M. M., de Titta, A., Jeanbart, L., and Hubbell, J. A. (2015) The TLR4 agonist fibronectin extra domain $A$ is cryptic, exposed by elastase-2; use in a fibrin matrix cancer vaccine. Sci. Rep. 5, 8569

68. Kohan, M., Muro, A. F., White, E. S., and Berkman, N. (2010) EDA-containing cellular fibronectin induces fibroblast differentiation through binding to a4ß37 integrin receptor and MAPK/Erk 1/2dependent signaling. FASEB J. 24, 4503-4512

69. Lefebvre, J. S., Lévesque, T., Picard, S., Paré, G., Gravel, A., Flamand, L., and Borgeat, P. (2011) Extra domain $A$ of fibronectin primes leukotriene biosynthesis and stimulates neutrophil migration through activation of Toll-like receptor 4. Arthritis Rheum. 63, 1527-1533

70. Kelsh, R., You, R., Horzempa, C., Zheng, M., and McKeown-Longo, P. J. (2014) Regulation of the innate immune response by fibronectin: synergism between the III-1 and EDA domains. PLoS One 9, e102974

71. Mamat, U., Woodard, R. W., Wilke, K., Souvignier, C., Mead, D., Steinmetz, E., Terry, K., Kovacich, C., Zegers, A., and Knox, C. (2013) Endotoxin-free protein production-ClearColi ${ }^{\mathrm{TM}}$ technology. Nat. Methods 10, 916

72. Hayes, C. S., and Williamson, H., Jr. (2001) Management of group A beta-hemolytic streptococcal pharyngitis. Am. Fam. Physician 63, 1557-1564

73. Neeman, R., Keller, N., Barzilai, A., Korenman, Z., and Sela, S. (1998) Prevalence of internalisation-associated gene, prtF1, among persisting group-A Streptococcus strains isolated from asymptomatic carriers. Lancet 352, 1974-1977

74. Stjernquist-Desatnik, A., Orrling, A., Schalén, C., and Kamme, C. (1992) Penicillin tolerance in group A streptococci and treatment failure in streptococcal tonsillitis. Acta Otolaryngol. 492, 68-71

75. Pichichero, M. E., Casey, J. R., Mayes, T., Francis, A. B., Marsocci, S. M., Murphy, A. M., and Hoeger, W. (2000) Penicillin failure in streptococcal tonsillopharyngitis: causes and remedies. Pediatr. Infect. Dis. J. 19, 917-923

76. Kaplan, E., Oakes, J., and Johnson, D. (2007) Unexpected Individual Clinical Site Variation in Eradication Rates of Group A Streptococci by Penicillin in Multisite Clinical Trials. Pediatr. Infect. Dis. J. 26, 1110-1116

77. Lancefield, R. C. (1959) Persistence of type-specific antibodies in man following infection with group A streptococci. J. Exp. Med. 110, 271-292 
78. Cunningham, M. W. (2000) Cross-reactive antigens of group A streptococci. in Gram-positive pathogens (Fischetti, V. A., Novick, R. P., Ferretti, J. J., Portnoy, D. A., and Rood, J. I. eds.), American Society for Microbiology Press, Washington, D.C. pp 66-77

79. Dale, J. B. (2008) Current status of group A streptococcal vaccine development. Adv. Exp. Med. Biol. 609, 53-63

80. Steer, A. C., Carapetis, J. R., Dale, J. B., Fraser, J. D., Good, M. F., Guilherme, L., Moreland, N. J., Mulholland, E. K., Schodel, F., and Smeesters, P. R. (2016) Status of research and development of vaccines for Streptococcus pyogenes. Vaccine 34, 2953-2958

81. Steer, A. C., Law, I., Matatolu, L., Beall, B. W., and Carapetis, J. R. (2009) Global emm type distribution of group A streptococci: systematic review and implications for vaccine development. The Lancet Infectious Diseases 9, 611-616

82. Guirguis, N., Fraser, D. W., Facklam, R. R., El Kholy, A., and Wannamaker, L. W. (1982) Typespecific immunity and pharyngeal acquisition of group A Streptococcus. Am. J. Epidemiol. 116, 933-939

83. Hoe, N., Lukomska, E., Musser, J., and Lukomski, S. (2007) Characterization of the immune response to collagen-like proteins Scl1 and Scl2 of serotype M1 and M28 group A Streptococcus. FEMS Microbiol. Lett. 277, 142-149

84. Peng, Y. Y., Yoshizumi, A., Danon, S. J., Glattauer, V., Prokopenko, O., Mirochnitchenko, O., Yu, Z., Inouye, M., Werkmeister, J. A., Brodsky, B., and Ramshaw, J. A. (2010) A Streptococcus pyogenes derived collagen-like protein as a non-cytotoxic and non-immunogenic cross-linkable biomaterial. Biomaterials 31, 2755-2761

85. McNitt, D. H., Van De Water, L., Marasco, D., Berisio, R., and Lukomski, S. (2018) Streptococcal collagen-like protein 1 binds wound fibronectin: implications in pathogen targeting. Curr. Med. Chem. 25, 1-1

86. Nizet, V., Ohtake, T., Lauth, X., Trowbridge, J., Rudisill, J., Dorschner, R. A., Pestonjamasp, V., Piraino, J., Huttner, K., and Gallo, R. L. (2001) Innate antimicrobial peptide protects the skin from invasive bacterial infection. Nature 414, 454-457

87. Dorschner, R. A., Pestonjamasp, V. K., Tamakuwala, S., Ohtake, T., Rudisill, J., Nizet, V., Agerberth, B., Gudmundsson, G. H., and Gallo, R. L. (2001) Cutaneous injury induces the release of cathelicidin anti-microbial peptides active against group A Streptococcus. J. Invest. Dermatol. 117, 91-97

88. Koide, A., Gilbreth, R. N., Esaki, K., Tereshko, V., and Koide, S. (2007) High-affinity singledomain binding proteins with a binary-code interface. Proceedings of the National Academy of Sciences 104, 6632-6637

89. Sha, F., Salzman, G., Gupta, A., and Koide, S. (2017) Monobodies and other synthetic binding proteins for expanding protein science. Protein science : a publication of the Protein Society 26, 910-924 
90. Koide, A., Wojcik, J., Gilbreth, R. N., Hoey, R. J., and Koide, S. (2012) Teaching an old scaffold new tricks: monobodies constructed using alternative surfaces of the FN3 scaffold. J. Mol. Biol. 415, 393-405

91. Vazquez-Lombardi, R., Phan, T. G., Zimmermann, C., Lowe, D., Jermutus, L., and Christ, D. (2015) Challenges and opportunities for non-antibody scaffold drugs. Drug Discov. Today 20, 1271-1283

92. Martin, H. L., Bedford, R., Heseltine, S. J., Tang, A. A., Haza, K. Z., Rao, A., McPherson, M. J., and Tomlinson, D. C. (2018) Non-immunoglobulin scaffold proteins: Precision tools for studying protein-protein interactions in cancer. New Biotechnol. 45, 28-35

93. Plückthun, A. (2015) Designed Ankyrin Repeat Proteins (DARPins): Binding Proteins for Research, Diagnostics, and Therapy. Annu. Rev. Pharmacol. Toxicol. 55, 489-511

94. Škrlec, K., Štrukelj, B., and Berlec, A. (2015) Non-immunoglobulin scaffolds: a focus on their targets. Trends Biotechnol. 33, 408-418

95. Koide, A., Bailey, C. W., Huang, X., and Koide, S. (1998) The fibronectin type III domain as a scaffold for novel binding proteins11Edited by J. Wells. J. Mol. Biol. 284, 1141-1151

96. Albrecht, M., Renneberg, H., Wennemuth, G., Moschler, O., Janssen, M., Aumuller, G., and Konrad, L. (1999) Fibronectin in human prostatic cells in vivo and in vitro: expression, distribution, and pathological significance. Histochem. Cell Biol. 112, 51-61

97. Katenkamp, K., Berndt, A., Hindermann, W., Wunderlich, H., Haas, K. M., Borsi, L., Zardi, L., and Kosmehl, H. (2004) mRNA expression and protein distribution of the unspliced tenascin-C isoform in prostatic adenocarcinoma. J. Pathol. 203, 771-779

98. Inufusa, H., Nakamura, M., Adachi, T., Nakatani, Y., Shindo, K., Yasutomi, M., and Matsuura, H. (1995) Localization of oncofetal and normal fibronectin in colorectal cancer. Correlation with histologic grade, liver metastasis, and prognosis. Cancer 75, 2802-2808

99. Hanamura, N., Yoshida, T., Matsumoto, E.-i., Kawarada, Y., and Sakakura, T. (1997) Expression of fibronectin and tenascin-C mRNA by myofibroblasts, vascular cells and epithelial cells in human colon adenomas and carcinomas. Int. J. Cancer 73, 10-15

100. Gopal, S., Veracini, L., Grall, D., Butori, C., Schaub, S., Audebert, S., Camoin, L., Baudelet, E., Radwanska, A., Beghelli-de la Forest Divonne, S., Violette, S. M., Weinreb, P. H., Rekima, S., Ilie, M., Sudaka, A., Hofman, P., and Van Obberghen-Schilling, E. (2017) Fibronectin-guided migration of carcinoma collectives. Nature Comm. 8, 14105

101. Lyons, A. J., Bateman, A. C., Spedding, A., Primrose, J. N., and Mandel, U. (2001) Oncofetal fibronectin and oral squamous cell carcinoma. Br. J. Oral Maxillofac. Surg. 39, 471-477

102. Marsh, T., Pietras, K., and McAllister, S. S. (2013) Fibroblasts as architects of cancer pathogenesis. Biochim. Biophys. Acta 1832, 1070-1078

103. Kalluri, R. (2016) The biology and function of fibroblasts in cancer. Nature Rev. Cancer 16, 582 
104. Gulubova, M., and Vlaykova, T. (2006) Immunohistochemical assessment of fibronectin and tenascin and their integrin receptors $\alpha 5 \beta 1$ and $\alpha 9 \beta 1$ in gastric and colorectal cancers with lymph node and liver metastases. Acta Histochem. 108, 25-35

105. Christofori, G. (2006) New signals from the invasive front. Nature 441, 444-450

106. Lu, P., Weaver, V. M., and Werb, Z. (2012) The extracellular matrix: A dynamic niche in cancer progression. J. Cell Biol. 196, 395-406

107. Nagaharu, K., Zhang, X., Yoshida, T., Katoh, D., Hanamura, N., Kozuka, Y., Ogawa, T., Shiraishi, T., and Imanaka-Yoshida, K. (2011) Tenascin C induces epithelial-mesenchymal transition-like change accompanied by SRC activation and focal adhesion kinase phosphorylation in human breast cancer cells. Am. J. Pathol. 178, 754-763

108. Ou, J., Peng, Y., Deng, J., Miao, H., Zhou, J., Zha, L., Zhou, R., Yu, L., Shi, H., and Liang, H. (2014) Endothelial cell-derived fibronectin extra domain A promotes colorectal cancer metastasis via inducing epithelial-mesenchymal transition. Carcinogenesis

109. Bae, Y. K., Kim, A., Kim, M. K., Choi, J. E., Kang, S. H., and Lee, S. J. (2013) Fibronectin expression in carcinoma cells correlates with tumor aggressiveness and poor clinical outcome in patients with invasive breast cancer. Hum. Pathol. 44, 2028-2037

110. Yang, Z.-T., Yeo, S.-Y., Yin, Y.-X., Lin, Z.-H., Lee, H.-M., Xuan, Y.-H., Cui, Y., and Kim, S.-H. (2016) Tenascin-C, a Prognostic Determinant of Esophageal Squamous Cell Carcinoma. PLoS One 11, e0145807

111. Spenlé, C., Saupe, F., Midwood, K., Burckel, H., Noel, G., and Orend, G. (2015) Tenascin-C: Exploitation and collateral damage in cancer management. Cell Adh. Mig. 9, 141-153

112. Strohl, W. R. (2018) Current progress in innovative engineered antibodies. Protein \& cell 9, 86120

113. Forbes, N. S., Coffin, R. S., Deng, L., Evgin, L., Fiering, S., Giacalone, M., Gravekamp, C., Gulley, J. L., Gunn, H., Hoffman, R. M., Kaur, B., Liu, K., Lyerly, H. K., Marciscano, A. E., Moradian, E., Ruppel, S., Saltzman, D. A., Tattersall, P. J., Thorne, S., Vile, R. G., Zhang, H. H., Zhou, S., and McFadden, G. (2018) White paper on microbial anti-cancer therapy and prevention. J Immunother. Cancer 6, 78

114. Linnebacher, M., Maletzki, C., Emmrich, J., and Kreikemeyer, B. (2008) Lysates of S. pyogenes serotype M49 induce pancreatic tumor growth delay by specific and unspecific antitumor immune responses. J. Immunother. 31, 704-713

115. Maletzki, C., Linnebacher, M., Kreikemeyer, B., and Emmrich, J. (2008) Pancreatic cancer regression by intratumoural injection of live Streptococcus pyogenes in a syngeneic mouse model. Gut 57, 483-491

116. Eisenstark, A., Kazmierczak, R. A., Dino, A., Khreis, R., Newman, D., and Schatten, H. (2007) Development of Salmonella strains as cancer therapy agents and testing in tumor cell lines. Methods Mol. Biol. 394, 323-354 
117. Dang, L. H., Bettegowda, C., Huso, D. L., Kinzler, K. W., and Vogelstein, B. (2001) Combination bacteriolytic therapy for the treatment of experimental tumors. Proceedings of the National Academy of Sciences 98, 15155-15160

118. Saw, P. E., Kim, S., Lee, I.-h., Park, J., Yu, M., Lee, J., Kim, J.-I., and Jon, S. (2013) Aptideconjugated liposome targeting tumor-associated fibronectin for glioma therapy. J Mat. Chem. B 1, 4723-4726

119. Han, Z., and Lu, Z.-R. (2017) Targeting fibronectin for cancer imaging and therapy. Journal of Materials Chemistry B 5, 639-654

120. Dobrovolskaia, M. A., and McNeil, S. E. (2007) Immunological properties of engineered nanomaterials. Nat Nano 2, 469-478

121. Caswell, C. C., Barczyk, M., Keene, D. R., Lukomska, E., Gullberg, D. E., and Lukomski, S. (2008) Identification of the first prokaryotic collagen sequence motif that mediates binding to human collagen receptors, integrins $\alpha 2 \beta 1$ and $\alpha 11 \beta 1$. J Biol Chem 283, 36168-36175

122. Naci, D., Vuori, K., and Aoudjit, F. (2015) $\alpha 2 \beta 1$ integrin in cancer development and chemoresistance. Semin. Cancer Biol. 35, 145-153

123. Navab, R., Strumpf, D., To, C., Pasko, E., Kim, K. S., Park, C. J., Hai, J., Liu, J., Jonkman, J., Barczyk, M., Bandarchi, B., Wang, Y. H., Venkat, K., Ibrahimov, E., Pham, N. A., Ng, C., Radulovich, N., Zhu, C. Q., Pintilie, M., Wang, D., Lu, A., Jurisica, I., Walker, G. C., Gullberg, D., and Tsao, M. S. (2016) Integrin [alpha]11[beta]1 regulates cancer stromal stiffness and promotes tumorigenicity and metastasis in non-small cell lung cancer. Oncogene 35, 1899-1908

124. Terao, Y. (2012) The virulence factors and pathogenic mechanisms of Streptococcus pyogenes. J. Oral Biosci. 54, 96-100

125. Spanier, J. G., Jones, S. J., and Cleary, P. (1984) Small DNA deletions creating avirulence in Streptococcus pyogenes. Science 225, 935-938

126. Scott, J. R., Cleary, P., Caparon, M. G., Heden, K. M. L., Musser, J. M., Hollingshead, S., and Podbielski, A. (1995) New name for the positive regulator of the M protein of group A Streptococcus. Mol. Microbiol. 17, 799

127. Rasmussen, M., Edén, A., and Björck, L. (2000) SclA, a novel collagen-like surface protein of Streptococcus pyogenes. Infect. Immun. 68, 6370-6377

128. Lukomski, S., Montgomery, C. A., Rurangirwa, J., Geske, R. S., Barrish, J. P., Adams, G. J., and Musser, J. M. (1999) Extracellular cysteine protease produced by Streptococcus pyogenes participates in the pathogenesis of invasive skin infection and dissemination in mice. Infect. Immun. 67, 1779-1788

129. Lukomski, S., Nakashima, K., Abdi, I., Cipriano, V. J., Shelvin, B. J., Graviss, E. A., and Musser, J. M. (2001) Identification and characterization of a second extracellular collagen-like protein made by group A Streptococcus: control of production at the level of translation. Infect. Immun. 69, $1729-1738$ 


\section{APPENDICES}

\section{APPENDIX 1: SUPPLEMENTARY MATERIAL FOR CHAPTER 1 SURFACE-EXPOSED LOOPS AND AN ACIDIC PATCH IN THE SCL1 PROTEIN OF GROUP A STREPTOCOCCUS ENABLE SCL1 BINDING TO WOUND-ASSOCIATED FIBRONECTIN}

Dudley H. McNitt, Soo Jeon Choi, Douglas R. Keene, Livingston Van De Water, Flavia Squeglia, Rita Berisio, Slawomir Lukomski Published in The Journal of Biological Chemistry 2018 293(20): 7796-7810.

Table S1: Synthetic double stranded DNA blocks (gBlocks) and primer nucleotide sequences used in this study.

\begin{tabular}{l} 
gBlock - pSL600 [rScl.chi1] \\
\hline ACACCATCGAATGGCCAGATGATTAATTCCTAATTTTTGTTGACACTCTATCATTG \\
ATAGAGTTATTTTACCACTCCCTATCAGTGATAGAGAAAAGGAATGAATAGTTC \\
GACAAAAATCTAGATAACGAGGGCAAAAAATGAAAAAGACAGCTATCGCGATTG \\
CAGTGGCACTGGCTGGTTTCGCTACCGTAGCGCAGGCCGATGAACAAGAAGAG \\
AAAGCTAAAGTTAGAACTGAATTAATTCAAGAGTTAGCTCAGAAATATCCAGAAG \\
TGTCCAATGAGAAATTTTGGGAAGAAAGTGGTATGGAACCTATTTAAAGAATT \\
AACATACCTACAGGAACGAGAACAAGCTGAGAATAGTTGGCGAAAAAGACTACT \\
AAAGGGTATACAAGATCATGCCCTTGATGGTCTCGCTGGTAAA \\
\hline gBlock - pSL613 [rScl.chi2] \\
ACACCATCGAATGGCCAGATGATTAATTCCTAATTTTTGTTGACACTCTATCATTG \\
ATAGAGTTATTTTACCACTCCCTATCAGTGATAGAGAAAAGTGAAATGAATAGTTC \\
GACAAAAATCTAGATAACGAGGGCAAAAATGAAAAAGACAGCTATCGCGATTG \\
CAGTGGCACTGGCTGGTTTCGCTACCGTAGCGCAGGCCGAGGTTTCTTCTACGA \\
CTATGACGTCGAGTCAAAGAGAGTCAAAAATAAAAGAGATCGAAGAAAGTCTTAA \\
AGGACTAGGGGGTATTGAGAAAAAAAATTTCCAACTCTAGGTGATGAAGATTTA \\
GATCATACTTATATGACAAAGCTAGAAGATTTTCAAAAGGAGCTAAAAGATTTTAC \\
TGAGAAGAGGCTTAAGGAGATTCTAGATTTAATTGGTAAATCTGGAATCAAGGGA \\
GACCGCGG \\
\hline gBlock - pSL614 [rSCI.chi3]
\end{tabular}


ACACCATCGAATGGCCAGATGATTAATTCCTAATTTTTGTTGACACTCTATCATTG ATAGAGTTATTTTACCACTCCCTATCAGTGATAGAGAAAAGTGAAATGAATAGTTC GACAAAAATCTAGATAACGAGGGCAAAAAATGAAAAAGACAGCTATCGCGATTG CAGTGGCACTGGCTGGTTTCGCTACCGTAGCGCAGGCCGATGAACAAGAAGAG AAAGCTAAAGTTAGAACTGAATTAATTCAAGAGTTAGCTCAGAAAGAATATCCAAA AGCGTCTGAGGAAAAATTTTGGGAGAGTAGCTTTTGGGGAAGAAGGTACTTTAAT GAATTAACATACCTACAGGAACGAGAACAAGCTGAGAATAGTTGGCGAAAAAGA CTACTAAAGGGTATACAAGATCATGCCCTTGATGGTCTCGCTGGTAAA

gBlock - pSL601 [rScl.chiN]

ACACCATCGAATGGCCAGATGATTAATTCCTAATTTTTGTTGACACTCTATCATTG ATAGAGTTATTTTACCACTCCCTATCAGTGATAGAGAAAAGTGAAATGAATAGTTC GACAAAAATCTAGATAACGAGGGCAAAAAATGAAAAAGACAGCTATCGCGATTG CAGTGGCACTGGCTGGTTTCGCTACCGTAGCGCAGGCCGATGAACAAGAAGAG AAAGCTAAAGTTAGAACTGAATTAATTCAAGAGTTAGCTCAGAAATATCCAGAAG TGTCCAATGAGAAATTTTGGACTCTAGGTGATGAAGATTTAGATCATACTTATTTA ACATACCTACAGGAACGAGAACAAGCTGAGAATAGTTGGCGAAAAAGACTACTA AAGGGTATACAAGATCATGCCCTTGATGGTCTCGCTGGTAAA

gBlock - pSL602 [rScl.chiC]

ACACCATCGAATGGCCAGATGATTAATTCCTAATTTTTGTTGACACTCTATCATTG ATAGAGTTATTTTACCACTCCCTATCAGTGATAGAGAAAAGTGAAATGAATAGTTC GACAAAAATCTAGATAACGAGGGCAAAAAATGAAAAAGACAGCTATCGCGATTG CAGTGGCACTGGCTGGTTTCGCTACCGTAGCGCAGGCCGATGAACAAGAAGAG AAAGCTAAAGTTAGAACTGAATTAATTCAAGAGTTAGCTCAGGGACTAGGGGGTA TTGAGAAAAAAAATTTTCCAGAAAGAAAGTGGTATGGAACCTATTTTAAAGAATTA ACATACCTACAGGAACGAGAACAAGCTGAGAATAGTTGGCGAAAAAGACTACTA AAGGGTATACAAGATCATGCCCTTGATGGTCTCGCTGGTAAA

gBlock - pSL603 [rScl.chil]

ACACCATCGAATGGCCAGATGATTAATTCCTAATTTTTGTTGACACTCTATCATTG ATAGAGTTATTTTACCACTCCCTATCAGTGATAGAGAAAAGTGAAATGAATAGTTC GACAAAAATCTAGATAACGAGGGCAAAAAATGAAAAAGACAGCTATCGCGATTG CAGTGGCACTGGCTGGTTTCGCTACCGTAGCGCAGGCCGATGAACAAGAAGAG AAAGCTAAAGTTAGAACTGAATTAATTCAAGAGTTAGCTCAGGGACTAGGGGGTA TTTCCAATGAGAAATTTTGGGAAAGAAAGTGGTATTTAGATCATACTTATATGTTA ACATACCTACAGGAACGAGAACAAGCTGAGAATAGTTGGCGAAAAAGACTACTA AAGGGTATACAAGATCATGCCCTTGATGGTCTCGCTGGTAAA

gBlock - pSL621 [Trans-complementation Scl.chi2]

CTCACCTTTTTCACCAGCTTTACCTTGGATTCCTTGCTCACCGCGGTCACCTTTA GGACCTTGGGCGCCCCTCTCACCAGTTTTACCTTGTGGTCCGGCTGGGCCAGC AGGACCAGTCTCACCACGGTCTCCCTTGATTCCAGATTTACCAATTAAATCTAGA ATCTCCTTAAGCCTCTTCTCAGTAAAATCTTTTAGCTCCTTTTGAAAATCTTCTAG CTTTGTCATATAAGTATGATCTAAATCTTCATCACCTAGAGTTGGAAAATTTTTTTT CTCAATACCCCCTAGTCCTTTAAGACTTTCTTCGATCTCTTTTATTTTTGACTCTCT TTGACTCGACGTCATAGTCGTAGAAGAAACCTCAGCCTTAACGCTGCTTGCCCC 


\begin{tabular}{|l|}
\hline GCCTCCAAAGGCTAGAAGGACGGCAGCGGCTGAGGTTAGCCCGTAGCGCCAGA \\
CTAGTTTGTTGAGATTATGGTGCTTTGATGTC \\
\hline Primer - pSL600_insert_1F \\
\hline ACACCATCGAATGGCCAGATG \\
\hline Primer - pSL600_insert_1R \\
\hline TTTACCAGCGAGACCATCAAGG \\
\hline Primer - pSL609_2R \\
\hline CCGCGGTCTCCCTTGATTCCA \\
\hline
\end{tabular}

(rScl.chi): recombinant chimeric streptococcal collagen-like protein. 


\title{
APPENDIX 2: MINI-REVIEW \\ STREPTOCOCCAL COLLAGEN-LIKE PROTEIN 1 BINDS WOUND FIBRONECTIN: IMPLICATIONS IN PATHOGEN TARGETING \\ McNitt DH, Van De Water L, Marasco D, Berisio R, Lukomski S \\ Published in Current Medicinal Chemistry 2018: Epub ahead of print
}

(Publication was part of invited review on biomarkers of disease. I wrote on the merits of utilizing the streptococcal collagen-like proteins 1 and 2 as biomarkers for group A Streptococcus infections)

\begin{abstract}
Group A Streptococcus (GAS) infections are responsible for significant morbidity and mortality worldwide. The outlook for an effective global vaccine is reduced because of significant antigenic variation among GAS strains worldwide. Other challenges in GAS therapy include the lack of common access to antibiotics in developing countries, as well as allergy to and treatment failures with penicillin and increasing erythromycin resistance in the industrialized world. At the portal of entry, GAS binds to newly deposited extracellular matrix, which is rich in cellular fibronectin isoforms with extra domain A (EDA, also termed EIIIA) via the surface adhesin, the streptococcal collagen-like protein 1 (Scl1). Recombinant Scl1 constructs, derived from diverse GAS strains, bind the EDA loop segment situated between the $C$ and $C$ ' $\beta$-strands. Despite the sequence diversity in Scl1 proteins, multiple sequence alignments and secondary structure predictions of Scl1 variants, as well as crystallography and homology modeling studies, point to a conserved mechanism of Scl1-EDA binding. We propose that targeting this interaction may prevent the progression of infection. A synthetic cyclic peptide, derived from the EDA C-C' loop, binds to recombinant Scl1 with a micromolar dissociation constant. This review highlights the current concept of EDA binding to Scl1 and provides incentives to exploit this binding to treat GAS infections and wound colonization.
\end{abstract}




\section{INTRODUCTION}

"A biomarker is any measurement reflecting an interaction between a biological system and a potential hazard, which may be chemical, physical, or biological. The measured response may be functional and physiological, biochemical at the cellular level, or a molecular interaction." (1). In the case of pathogens, typical biomarkers are genes or surface proteins/components that are expressed during the infection. Biomarker identification and characterization aid in the understanding of pathogenesis and can be used for diagnosis or treatment (2), e.g., the surface adhesin HBHA of Mycobacterium tuberculosis, as biomarker to distinguish between latent and active tuberculosis infections (3). The identification of novel biomarkers is a compelling need for many bacterial pathogens, including highly virulent bioterrorism threat pathogens, such as Francisella tularensis and Burkholderia spp. $(4,5)$, or pathogens with antibiotic resistance concerns, such as Staphylococcus aureus and group A Streptococcus (6).

Group A Streptococcus (GAS) is a human-adapted pathogen that is responsible for over 700 million infections worldwide each year (7). Diseases linked to GAS infection range in severity from non-invasive superficial pharyngitis and impetigo to severe and invasive diseases like necrotizing fasciitis or streptococcal toxic shock syndrome (7). Additionally, post-infectious autoimmune sequelae include rheumatic fever and rheumatic heart disease, acute poststreptococcal glomerulonephritis, and pediatric autoimmune neurologic disorder associated with streptococci, also known as PANDAS (8-10). Invasive and autoimmune diseases account for approximately 500,000 deaths globally each year (7). GAS can transiently colonize barrier tissue sites, such as throat mucosa and the surface of the skin (11). When the barrier is breached, the resultant wound provides GAS with a portal of entry into host tissues and the interstitium. Once inside the wound, GAS adheres to exposed extracellular matrix (ECM) and cells, thus enabling colonization of the host. These essential first steps of infection that are critical to colonization are augmented by several GAS surface adhesins, such as a prominent GAS surface adhesin and key virulence factor, the M protein (12). DNA sequence encoding the outermost amino-terminal end of the $M$ protein is hypervariable and is used for GAS typing, resulting in more than 220 emm types of GAS reported globally (13). Two other major GAS surface proteins are the streptococcal collagen-like proteins 1 (Scl1/SclA) and 2 (Scl2/SclB), 
which are ubiquitous among GAS strains (14-18). In vitro assays and animal model experiments show that Scl1 contributes to host colonization and biofilm formation within tissues by binding to host extracellular matrix proteins, cellular fibronectin (cFn), and laminin $(19,20)$. Scl1 binds to wound-enriched isoforms of $c F n$, containing the extra domain A (EDA, also termed EIIIA), which is a unique target and distinct from other GAS fibronectin adhesins (21). The ubiquitous presence of Scl1 in GAS strains across different $M$ types and the distinctive binding to the EDA/cFn isoforms within the wound microenvironment make Scl1 a potential biomarker for GAS infections.

GAS infections are initially identified in physician's offices, using a Rapid Antigen Detecting Test based on the original Lancefield's concept to detect group A carbohydrate surface antigen (22). More recent methods target nucleic acids, such as GASDirect hybridization test, which identifies rRNA sequences specific to $S$. pyogenes in tissue swabs (23), and a PCR-based method was developed to detect GAS within clinical isolates (24); more information can be found elsewhere (25). In this review, we will discuss the merit of Scl1 and Scl2 proteins as potential GAS biomarkers, their unique functions in GAS pathogenesis, and strategies to utilize the Scl1-EDA interaction to target diverse and epidemiologically important GAS M types. The long-term goal is to disrupt wound colonization and prevent the spread of infection.

\section{SCL1 AND SCL2 AS BIOMARKERS OF GAS INFECTION}

A hallmark of a pathogen biomarkers is that it is expressed by all strains of the organism and during critical stages of infection. Both $s c / 1$ and $s c / 2$ genes have been identified in many epidemiologically important $M$ types of GAS $(14-17,26)$. sc/1 expression is positively regulated by the multiple-gene regulator, Mga $(14,15,27)$. Mga is a major virulence-associated transcriptional regulator expressed in all GAS strains (28-31). It controls the expression of key GAS virulence factors, including the $M$ and $M$-like proteins, C5a peptidase, Sic, and Scl1 (14,15,32-34). Elevated transcription of $s c / 1$ has been found in GAS isolates from both non-invasive and invasive infections $(26,35)$, underscoring the premise of Scl1 as a biomarker. Unlike Scl1, expression of full-length Scl2 protein is controlled during polypeptide translation by phase variation, which is associated with varying numbers of pentanucleotide CAAAA repeats located downstream of the scl2-start 
codon (15-17). Like scl1, scl2 transcription has been observed during GAS infection, but protein expression varies between strains due to the aforementioned phase variation $(15,36)$. The invasive M3-type GAS strains (37-39) show elevated levels of scl2 transcripts and low levels of $s c / 1$ transcripts, compared with strains of several other $\mathrm{M}$ types (40). Therefore, Scl2 protein is a better biomarker in M3 organisms.

Detection of specific antibodies elicited during infection is commonly used in diagnostic assays. Host seroconversion to Scl1 and Scl2 antigens has been documented in humans and experimental murine models. Convalescent and acute-phase sera were positive for anti-Scl1 and anti-Scl2 antibodies following infections with M1-type and M28type strains. M1 GAS is a global pandemic M type, linked with both noninvasive and invasive infections across the globe $(41,42)$. M28 GAS strains have historically been associated with puerperal sepsis and neonatal infections (43-45), but also with current cases of pharyngitis and invasive infections worldwide (46-49). Retrospective examination of sera from patients with known previous infection by M1 GAS strains detected anti-Scl 1 antibodies in $64 \%$ of samples (50). Examination of acute and convalescent sera from pediatric patients with non-invasive M1 and M28 GAS infections identified broad presence of anti-Scl1 antibodies, whereas anti-Scl2 antibodies associated with M28 infections were detected with lower frequency; the Scl2 antigen is not expressed in M1 GAS strains due to an off-phase effect associated with CAAAA repeats $(15,40)$. Others have reported the presence of both anti-Scl1 and anti-Scl2 antibodies in patient sera infected by GAS strains of various $M$ types that were collected from cases of pharyngitis and impetigo, as well as acute rheumatic fever $(51)^{1}$. Interestingly, antibody levels against Scl1 and Scl2 antigens in patients suffering from severe and invasive infections (toxic shock and necrotizing fasciitis) with $M 1, M 3$, and M28 GAS were significantly lower than in patients with non-severe bacteremia, suggesting that humoral adaptive immunity plays an important role in the protection against severe invasive diseases (52). In murine models of pharyngitis and impetigo, mice administered intranasally and subcutaneously, respectively, with M1 and M28 strains seroconverted to Scl1 antigen, apparently expressed during infections (50).

\footnotetext{
${ }^{1}$ SL unpublished data
} 
Comparatively, there was moderate seroconversion for Scl2 antigen in mice infected with M28 GAS. Altogether, utilization of anti-Scl1 and anti-Scl2 antibodies as biomarkers depends on the type of GAS infection (e.g., throat versus skin infection and superficial versus invasive infection). Furthermore, variation in the primary Scl1 and Scl2 sequences across GAS strains pose additional diagnostic challenge. Alternative approaches, utilizing ligands that target Scl1 and Scl2 variants in multiple M types could circumvent these challenges.

\subsection{SCL1 AND SCL2 STRUCTURE}

Both Scl1 and Scl2 proteins have similar structural organization (53) (Figure 1A). Both are homotrimeric, with an $\mathrm{N}$-terminal globular, sequence-variable $(\mathrm{V})$ domain, followed by the collagen-like $(C L)$ domain and a C-terminal cell wall-anchoring domain. The $C L$ domain of both Scl1 and Scl2 is composed of varying numbers of Gly-Xaa-Yaa collagen repeats, analogous to triple-helical mammalian collagen $(54,55)$. Structurally, the triple helix is a motif characterized by the association of three distinct polypeptide chains wrapped around a common axis, where each chain assumes a polyproline-II (PPII)-type conformation (56-59). However, the CL domain does not contain hydroxyproline, which provides the main stabilizing contribution to triple-helix structure in mammalian collagens (60-64). Indeed, bacteria lack the prolyl-hydroxylase, an enzyme necessary for posttranslational modification of Pro to Hyp. Despite the lack of Hyp, however, Scl1- and Scl2$C L$ domains form triple-helix structures with a stability ranging between 35 and $40^{\circ} \mathrm{C}$, similar to mammalian collagens $(54,65-68)$. Instead, alternative stabilization mechanisms are employed in bacteria that secure triple-helix stability, such as hydration networks, electrostatic interactions between side chains, and specific stabilization repeats $(55,68)$.

The primary sequences of $\mathrm{V}$ domains differ both between and within Scl1 and Scl2 variants originating from strains of different $M$ types (53). Despite sequence differences, secondary-structure prediction algorithms identified two conserved a-helices interspaced by a hypervariable segment $(14,69)$. This prediction was validated by crystallography data of the recombinant protein, corresponding to the Scl2-V domain from an M3-type GAS $(70,71)$ (Figure $1 \mathrm{~A})$. The crystal structure showed that the Scl2-V domain folds into a trimeric six-helical bundle, forming a cylinder that is $30 \AA$ in diameter and $60 \AA$ in height, 
which is stabilized by a hydrophobic core and salt bridges. The inner core of this bundle consists of a parallel, trimeric structure in which helices are wrapped in a gradual lefthanded superhelix. Three further helices wrap antiparallel to the internal helices in a lefthanded direction around the exterior of the central trimer. The pairs of anti-parallel $\alpha$ helices are each connected with PPII-type loop segments. Structural and biophysical data suggested that $\mathrm{V}$ domains of Scls display dichotomous functions by first acting as triplehelix stabilization domains through facilitating proper folding and refolding of the collagen triple-helix (72) and second, by mediating host-pathogen interaction (73-75). Consistently, recombinant collagen-domain constructs cannot refold following thermal denaturation $(54,72)$.

This crystal structure served as the basis for homology models of sequencediverse Scl1- and Scl2-V domains (76) (Figure 1B). Homology models predicted with confidence that both the Scl1- and Scl2-V domains adopt similar six-helical bundle structures, where a flexible loop segment connects each $\alpha$-helix pair. Crystal structure and homology models revealed two important features found in Scl1- and Scl2-V domains that are essential for EDA-fibronectin binding. First, the electrostatic potential surface of ligand-binding-positive Scl1 protein displays a negatively charged region in the center of the Scl1-V trimer, which is surrounded by neutral and positively charged residues. In contrast, the electrostatic surface of the binding-negative Scl2 protein displays a uniform negative surface charge throughout the crown of the Scl2-V domain (76). The arrangement of the electrostatic surface potential observed in the Scl1-V domains was associated with a pattern of conserved negatively charged amino acids, primarily $E^{46}$,

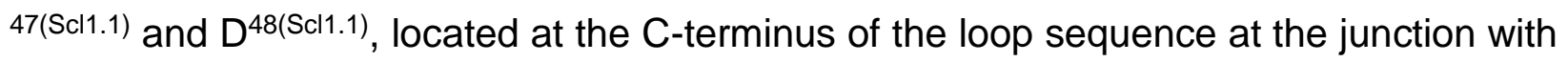
the $\alpha$-helices forming the inner core of the Scl1.1-V bundle. Second, productive ligand binding is further due to the surface-exposed loops of the Scl1-V domain. Surfaceexposed loops and a characteristic distribution of charged residues are proposed as the key molecular determinants that generate the long-range interactions needed for ligand recognition and binding. 


\subsection{FIBRONECTIN WITHIN THE WOUND MICROENVIRONMENT}

Scl1 binds to several ligands found in tissue and blood, compared with Scl2; the latter's function in GAS pathogenesis is less clear (reviewed in (53)). The collagen domain of Scl1 can bind and activate human collagen integrin receptors $(73,74,77)$, whereas the $V$ domain is responsible for the majority of ligand binding (19,69,77-80). Interestingly, Scl1 mutants of M1-type GAS were attenuated in a mouse skin infection model, but not following intraperitoneal inoculation(18), conceivably due to differences in the capacity to evade innate immune defenses between wild type and mutant strains $(18,81)$. In contrast, Scl1 mutants of two other M types exhibited increased lesion size in mouse skin (40). Here we focus on the interaction between Scl1 protein and the human ECM protein, cellular fibronectin $(19,21)$.

GAS utilizes a wounded portal of entry to gain access to host tissues, initiating host colonization and subsequent infection. One of the essential measures during wound healing is the deposition of new distinct matrices that participate in tissue remodeling and signal pro-inflammatory responses. These complex processes engage macrophages and neutrophils in clearing out tissue debris and keratinocytes in facilitating wound closure $(82,83)$. One of the major constituents of these matrices is fibronectin (Fn) (84-86). Fn is a high-molecular weight glycoprotein that exists in two main forms within the body, e.g., as a plasma fibronectin ( $\mathrm{pFn}$ ) secreted by hepatocytes into the blood stream and as a cellular fibronectin (cFn) secreted by cells within tissues (87). Both forms of fibronectin originate from the same gene, $F N 1$, and are composed of three different types of repeating domains: types I, II, and III $(87,88)$ (Figure $2 A)$. cFn-mRNA can be alternatively spliced, resulting in more than 20 different isoforms found within the body (88-90). This alternative splicing leads to the inclusion of extra domains that are found in $\mathrm{cFn}$, but not pFn. The alternatively spliced type III domains are known as extra domain A (EDA/EIIIA) and extra domain $B(E D B / E I I B)$, as well as different combinations of the type III connecting segment (IIICS), also known as the variable domain (90). The EDA-containing cFn isoforms (EDA/cFn) are highly expressed during embryonic development but only at negligible levels within healthy adult tissues (91-93). On the other hand, EDA/cFn isoforms are deposited in high quantities within inflamed and wounded tissue environments in adults and play essential roles in wound healing (92,94-96). EDA adopts 
the prototypical type III repeat structure, which is composed of two $\beta$-sheets, forming a $\beta$ sandwich consisting of seven $\beta$-strands (i.e. A, B, C, C', D, E, and F) connected by loop segments $(97,98)$ (Figure $2 \mathrm{~B}$ ). An important role for EDA/cFn within wounded tissue is to support host cell attachment, achieved by the interactions between the C-C' loop of EDA and cellular integrin receptors, $\alpha_{9} \beta_{1}$ and $\alpha_{4} \beta_{1}$, as well as $\alpha_{4} \beta_{7}$, which binds EDA by an unknown mechanism (99-101). Integrin $\alpha_{9} \beta_{1}$ is upregulated by keratinocytes during tissue injury $(94,102)$. Integrins $\alpha_{9} \beta_{1}, \alpha_{4} \beta_{1}$, and $\alpha_{4} \beta_{7}$ through their attachment to EDA/cFn regulate important repair mechanisms, such as cell migration, angiogenesis, myofibroblast differentiation, growth factor secretion, and tissue remodeling $(94,96,101,103,104)$.

Wound keratinocytes express $\alpha_{9} \beta_{1}$, which exerts a "trans-dominant" inhibitory effect on $\alpha_{3} \beta_{1}$ during angiogenesis in healing wounds (96). On keratinocytes, $\alpha_{3} \beta_{1}$ binds to laminin-332, and this binding mediates endothelial cell migration and angiogenesis through the secretion of growth factors $(105,106)$. Binding of $\alpha_{9} \beta_{1}$ to EDA/cFn controls $\alpha_{3} \beta_{1}$-mediated angiogenesis by modulating growth factor secretion, thereby reducing endothelial cell migration and survival during angiogenesis (96). Inhibition of $\alpha_{3} \beta_{1}$ by $\alpha_{9} \beta_{1}$ occurs through changes in Src-mediated phosphorylation of focal adhesion kinase (96). The temporal expression of EDA/cFn, during wound healing, suggests that the $\alpha_{9} \beta_{1}$-to$\alpha_{3} \beta_{1}$ integrin cross-talk is important for controlling the level of angiogenesis. Given that Scl1 and integrin $\alpha_{9} \beta_{1}$ bind to the same peptide region of the EDA segment, these findings suggest that $\alpha_{3} \beta_{1}$-mediated effects in keratinocytes could be perturbed by Scl1-mediated streptococcal adhesion.

One of the critical steps of wound healing is tissue remodeling, which is fostered by matrix metalloproteinases, which are secreted by both resident tissue cells and infiltrating immune cells $(86,107)$. EDA/cFn fragments generated by this proteolysis activate toll-like receptor 4 (TLR4) on dermal fibroblasts, as well as monocytes and dendritic cells $(101,107,108)$. This interaction induces the NF-KB signaling cascade, promoting pro-inflammatory responses, such as production of cytokines IL-8 and TNFa (108). EDA-mediated activation of TLR4 is dependent on the TLR4-co-receptor, MD-2, and evidence suggests that activation of TLR4 is enhanced by synergy from neighboring Fn domains (107-109). 


\subsection{SCL1 BINDING TO WOUND FIBRONECTIN}

The Scl1 proteins of several different M types of GAS bind to the C-C' loop of EDA, promoting GAS wound-specific recognition and tissue microcolony (in vivo biofilm) formation (21). The Scl1-EDA interaction is different from other fibronectin-binding proteins of GAS, such as PrtF1 and F2, Fba, FBp54, etc. These proteins bind to the Nterminal fibronectin module composed of type I repeats, which is found in both $\mathrm{pFn}$ and cFn (110-113) (Figure 2A). Adherence to the N-terminal type I repeats occurs through a $\beta$-zipper mechanism, where the adhesin contributes an additional $\beta$-strand to one of the $\beta$-sheets of the type I Fn repeat (112). In contrast, Scl1 binding to the type III repeat of cFn, the EDA, engages the surface-exposed loops and negatively charged cleft of the Scl1-V domain (76).

Although not demonstrated experimentally, the Scl1-EDA/cFn interaction may alter normal wound-healing responses. First, Scl1 binding to EDA was mapped to the C-C' loop (21) at or near the binding site of interaction with host cell integrin receptor $\alpha_{9} \beta_{1}$ (99). By competing with the host's integrins, Scl1 binding to EDA/cFn could lead to alterations of the dynamic wound-healing process, such as changes in angiogenesis (Figure 3). In addition, Scl1 could prevent or delay the formation of fibrosis by blocking $\alpha_{4} \beta_{1}$-mediated attachment to EDA/cFn. It is currently not known, whether Scl1 binding to EDA affects TLR4 function. In such a scenario, Scl1 action could reduce the production of proinflammatory cytokines and infiltration of neutrophils to the site of infection, thereby adding a new immune evasion strategy to the GAS repertoire (Figure 3). Ongoing experiments will test this hypothesis. Altogether, Scl1 plays essential roles in the pathogenesis of GAS and has the potential to alter host functions during infection.

\section{DIFFERENT STRATEGIES FOR TARGETING GAS INFECTIONS}

Currently, GAS infections are treated either with penicillin or erythromycin in patients with a penicillin allergy (114). Alarmingly, several studies documented penicillin treatment failure, with up to $30 \%$ of pharyngitis patients showing persistence of GAS following treatment (115-117). Additionally, there is an increasing frequency of erythromycin 
resistance among GAS strains circulating in the industrialized world $(118,119)$, again underscoring the need for new alternative strategies to treat GAS infection.

Considerable efforts have been undertaken by research laboratories in search of vaccine targets to prevent GAS infections and the development of a universal GAS vaccine. The immunogenic surface $M$ protein serves as the basis for most GAS vaccine candidates, as it confers M type-specific immunity (120). Historically, cross-reactive antibody concerns between M protein and cardiac tissue impeded development of GAS vaccines for years; however, current methods utilize peptides corresponding to the $\mathrm{N}$ terminal hypervariable region, thereby avoiding autoreactive portions $(121,122)$. A 26 valent vaccine, which is composed of $\mathrm{N}$-terminal hypervariable region peptides derived from 26 different M-protein types, has completed stage I/II clinical trials in the United States (123). Still, other challenges exist, such as the high number of M protein types, the lack of vaccine protection from throat infections, and differences in M-type distribution across the globe $(124,125)$.

Antibodies against the Scl1 antigen may possibly be developed as a strategy to treat GAS infections. In general, anti-Scl1 antibodies could be opsonic or may well block the interactions between Scl1 and EDA/cFn, thus, preventing GAS colonization. Although infection with GAS can lead to the production of anti-Scl1 cross-reactive autoantibodies (outlined in section 2), others have found that recombinant Scl1-based collagen proteins are non-immunogenic (126). Variability of the amino acid sequence of the Scl1 V-domain would face challenges similar to those concerning the $M$ protein-based vaccine. Therefore, targeting GAS with C-C' loop-derived peptides could hypothetically overcome this hurdle.

\subsection{POTENTIAL PROBLEMS AND SOLUTIONS}

Inasmuch as utilizing peptides derived from the C-C' loop of EDA is a rational premise for treating GAS infections, there are significant challenges that need to be overcome.

\subsubsection{BINDING AFFINITIES}

First, the binding affinities between C-C'-loop circular peptide and two different recombinant Scl1 variants, originating from M1- and M3-type GAS, were in a range of 
40-60 $\mu \mathrm{M} K_{D}$, as estimated through surface plasmon resonance and tryptophan fluorescence binding assays (Fig. 2 C) (40). This binding is relatively weak, especially when compared with the binding affinity of an antibody. EDA-derived peptides can be improved for Scl1 binding by first systematically determining the minimum amino acids involved in the critical binding, using alanine scanning mutagenesis. After the minimum

pharmacophore is found, then synthetic modifications of peptidomimetics can be generated and tested for binding; these compounds will incorporate natural and unnatural amino acids (e.g., D-amino acid, $\alpha$ - $\alpha$-disubstituted amino acids, macrocycles, etc.) (127129).

\subsubsection{OFF-TARGET BINDING EFFCTS}

Second, off-target binding effects of the C-C' loop peptide directly to host cell integrin receptor $\alpha_{9} \beta_{1}$ could lead to dysregulation of the dynamic wound-healing processes, such as dysregulation in tissue angiogenesis (Figure 3). To address these challenges, the optimized C-C' loop peptide must be tested for the potential recognition by host cell integrins and other receptors.

\subsubsection{STRAINS THAT DO NOT EXPRESS SCL1}

Third, although all strains carry the sc/1 gene, a small subset of strains do not express Scl1, such as M3-type GAS (40). In these strains, however, Scl2 protein would serve as an alternative target.

\subsubsection{INCREASED INVASIVENESS}

Fourth, the C-C' loop-peptide could increase bacterial spread via microcolony dispersion. Nevertheless, sessile GAS cells will be susceptible to drugs or C-C' loop-derived peptide conjugated to an antimicrobial peptide, like LL-37 $(130,131)$.

\section{CONCLUSIONS}

- The scl1 and scl2 genes are found in every strain of GAS, and both Scl1 and Scl2 proteins elicit humoral immunity during infection. These factors make Scl1 and Scl2 promising biomarkers of GAS infection. 
- The Scl1-V domain structure is conserved among diverse GAS M types, and several rScl1 constructs bind to the C-C' loop of EDA through the same mechanism. The conserved Scl1 structure and function in strains of epidemiologically important $M$ types serve as the foundation for effective targeting of GAS.

- EDA/cFn is highly upregulated within wounded tissues and plays important healing roles. Scl1 binds to the C-C' loop of EDA, and this interaction stimulates wound-specific colonization of GAS, by facilitating pathogen adherence and tissue microcolony formation. Scl1 binding probably impedes the important wound-healing functions that are normally executed by EDA/cFn isoforms

\section{ACKNOWLEGEMENTS}

We thank Nyles Charon for critical reading of the manuscript. We thank Soo Jeon Choi and previous laboratory members for their contributions in procurement of data for the manuscript. This work was supported in part by: (DHM) the Integrative Graduate Education and Research (IGERT) Traineeship for Research and Education in Nanotoxicology under Award 1144676 and the Jennifer Gossling Scholarship in Microbiology; (SL) National Institutes of Health Grants AI50666 and Al083683 as well as West Virginia University HSC Bridge Grant Funding.

\section{REFERENCES}

1. Strimbu, K., and Tavel, J. A. (2010) What are biomarkers? Current opinion in HIV and AIDS 5, 463-466

2. Hsueh, C. T., Liu, D., and Wang, H. (2013) Novel biomarkers for diagnosis, prognosis, targeted therapy and clinical trials. Biomarker Research 1, 1

3. Squeglia, F., Ruggiero, A., De Simone, A., and Berisio, R. (2018) A structural overview of mycobacterial adhesins: Key biomarkers for diagnostics and therapeutics. Protein Sci. 27, 369380

4. Durighello, E., Bellanger, L., Ezan, E., and Armengaud, J. (2014) Proteogenomic biomarkers for identification of Francisella species and subspecies by matrix-assisted laser desorption ionization-time-of-flight mass spectrometry. Anal. Chem. 86, 9394-9398

5. Bachert, B. A., Choi, S. J., Snyder, A. K., Rio, R. V. M., Durney, B. C., Holland, L. A., Amemiya, K., Welkos, S. L., Bozue, J. A., Cote, C. K., Berisio, R., and Lukomski, S. (2015) A unique set of 
the Burkholderia collagen-like proteins provides insight into pathogenesis, genome evolution and niche adaptation, and infection detection. PLoS One 10, e0137578

6. Solomon, S. L., and Oliver, K. B. (2014) Antibiotic resistance threats in the United States: stepping back from the brink. Am. Fam. Physician 89, 938-941

7. Carapetis, J. R., Steer, A. C., Mulholland, E. K., and Weber, M. (2005) The global burden of group A streptococcal diseases. Lancet Infect. Dis. 5, 685-694

8. Bisno, A. L., Pearce, I. A., Wall, H. P., Moody, M. D., and Stollerman, G. H. (1970) Contrasting epidemiology of acute rheumatic fever and acute glomerulonephritis. N. Engl. J. Med. 283, 561565

9. Swedo, S. E., Leonard, H. L., Mittleman, B. B., Allen, A. J., Rapoport, J. L., Dow, S. P., Kanter, M. E., Chapman, F., and Zabriskie, J. (1997) Identification of children with pediatric autoimmune neuropsychiatric disorders associated with streptococcal infections by a marker associated with rheumatic fever. Am. J. Psychiatry 154, 110-112

10. Cunningham, M. W. (2000) Pathogenesis of group A streptococcal infections. Clin. Microbiol. Rev. 13, 470-511

11. Bessen, D. E., and Lizano, S. (2010) Tissue tropisms in group A streptococcal infections. Future Microbiol. 5, 623-638

12. Fischetti, V. (2016) M Protein and Other Surface Proteins on Streptococci. in Streptococcus pyogenes : Basic Biology to Clinical Manifestations [Internet] (Ferretti JJ, S. D., Fischetti VA ed.), Univeristy of Oklahoma Health Sciences Center, Oklahoma City, OK. pp

13. Sanderson-Smith, M., De Oliveira, D. M., Guglielmini, J., McMillan, D. J., Vu, T., Holien, J. K., Henningham, A., Steer, A. C., Bessen, D. E., Dale, J. B., Curtis, N., Beall, B. W., Walker, M. J., Parker, M. W., Carapetis, J. R., Van Melderen, L., Sriprakash, K. S., and Smeesters, P. R. (2014) A systematic and functional classification of Streptococcus pyogenes that serves as a new tool for molecular typing and vaccine development. J. Infect. Dis. 15; 1325-38

14. Rasmussen, M., Edén, A., and Björck, L. (2000) SclA, a novel collagen-like surface protein of Streptococcus pyogenes. Infect. Immun. 68, 6370-6377

15. Lukomski, S., Nakashima, K., Abdi, I., Cipriano, V. J., Shelvin, B. J., Graviss, E. A., and Musser, J. M. (2001) Identification and characterization of a second extracellular collagen-like protein made by group A Streptococcus: control of production at the level of translation. Infect. Immun. 69, 1729-1738

16. Rasmussen, M., and Björck, L. (2001) Unique regulation of SclB - a novel collagen-like surface protein of Streptococcus pyogenes. Mol. Microbiol. 40, 1427-1438

17. Whatmore, A. M. (2001) Streptococcus pyogenes sclB encodes a putative hypervariable surface protein with a collagen-like repetitive structure. Microbiology 147, 419-429

18. Lukomski, S., Nakashima, K., Abdi, I., Cipriano, V. J., Ireland, R. M., Reid, S. D., Adams, G. G., and Musser, J. M. (2000) Identification and characterization of the $s c /$ gene encoding a group $A$ 
Streptococcus extracellular protein virulence factor with similarity to human collagen. Infect. Immun. 68, 6542-6553

19. Caswell, C. C., Oliver-Kozup, H., Han, R., Lukomska, E., and Lukomski, S. (2010) Scl1, the multifunctional adhesin of group A Streptococcus, selectively binds cellular fibronectin and laminin, and mediates pathogen internalization by human cells. FEMS Microbiol. Lett. 303, 61-68

20. Oliver-Kozup, H. A., Elliott, M., Bachert, B. A., Martin, K. H., Reid, S. D., Schwegler-Berry, D. E., Green, B. J., and Lukomski, S. (2011) The streptococcal collagen-like protein-1 (Scl1) is a significant determinant for biofilm formation by group A Streptococcus. BMC Microbiol. 11, 262

21. Oliver-Kozup, H., Martin, K. H., Schwegler-Berry, D., Green, B. J., Betts, C., Shinde, A. V., Van De Water, L., and Lukomski, S. (2013) The group A streptococcal collagen-like protein-1, Scl1, mediates biofilm formation by targeting the extra domain A-containing variant of cellular fibronectin expressed in wounded tissue. Mol. Microbiol. 87, 672-689

22. Lancefield, R. C. (1933) A SEROLOGICAL DIFFERENTIATION OF HUMAN AND OTHER GROUPS OF HEMOLYTIC STREPTOCOCCI. J. Exp. Med. 57, 571-595

23. Steed, L. L., Korgenski, E. K., and Daly, J. A. (1993) Rapid detection of Streptococcus pyogenes in pediatric patient specimens by DNA probe. J. Clin. Microbiol. 31, 2996-3000

24. Anderson, N. W., Buchan, B. W., Mayne, D., Mortensen, J. E., Mackey, T.-L. A., and Ledeboer, N. A. (2013) Multicenter Clinical Evaluation of the illumigene Group A Streptococcus DNA Amplification Assay for Detection of Group A Streptococcus from Pharyngeal Swabs. J. Clin. Microbiol. 51, 1474-1477

25. Spellerberg, B., Brandt C. . (2016) Laboratory Diagnosis of Streptococcus pyogenes (group A streptococci). in Streptococcus pyogenes : Basic Biology to Clinical Manifestations (Ferretti JJ, S. D., Fischetti VA ed., University of Oklahoma Health Sciences Center, Internet

26. Sumby, P., Whitney, A. R., Graviss, E. A., DeLeo, F. R., and Musser, J. M. (2006) Genome-wide analysis of group A streptococci reveals a mutation that modulates global phenotype and disease specificity. PLoS Pathog. 2, e5

27. Almengor, A. C., and Mclver, K. S. (2004) Transcriptional activation of sclA by Mga requires a distal binding site in Streptococcus pyogenes. J. Bacteriol. 186, 7847-7857

28. Spanier, J. G., Jones, S. J., and Cleary, P. (1984) Small DNA deletions creating avirulence in Streptococcus pyogenes. Science 225, 935-938

29. Podbielski, A. (1992) Ubiquitous occurrence of virR and $s c p A$ genes in group A streptococci. Med. Microbiol. Immunol. 181, 227-240

30. Scott, J. R., Cleary, P., Caparon, M. G., Heden, K. M. L., Musser, J. M., Hollingshead, S., and Podbielski, A. (1995) New name for the positive regulator of the M protein of group A Streptococcus. Mol. Microbiol. 17, 799 
31. Bessen, D., Manoharan, A., Luo, F., Wertz, J., and Robinson, D. (2005) Evolution of transcription regulatory genes is linked to niche specialization in the bacterial pathogen Streptococcus pyogenes. J. Bacteriol. 187, 4163-4172

32. Caparon, M. G., and Scott, J. R. (1987) Identification of a gene that regulates expression of M protein, the major virulence determinant of group A streptococci. Proc. Natl. Acad. Sci. U. S. A. 84, 8677-8681

33. Hoe, N. P., Nakashima, K., Lukomski, S., Grigsby, D., Liu, M., Kordari, P., Dou, S.-J., Pan, X., Vuopio-Varkila, J., Salmenlinna, S., McGeer, A., Low, D. E., Schwartz, B., Schuchat, A., Naidich, S., De Lorenzo, D., Fu, Y.-X., and Musser, J. M. (1999) Rapid selection of complement-inhibiting protein variants in group A Streptococcus epidemic waves. Nat. Med. 5, 924-929

34. Podbielski, A. (1993) Three different types of organization of the vir regulon in group A streptococci. Mol. Gen. Genet. 237, 287-300

35. Tsatsaronis, J. A., Hollands, A., Cole, J. N., Maamary, P. G., Gillen, C. M., Ben Zakour, N. L., Kotb, M., Nizet, V., Beatson, S. A., Walker, M. J., and Sanderson-Smith, M. L. (2013) Streptococcal collagen-like protein $A$ and general stress protein 24 are immunomodulating virulence factors of group A Streptococcus. FASEB J. 27, 2633-2643

36. Cole, J. N., Ramirez, R. D., Currie, B. J., Cordwell, S. J., Djordjevic, S. P., and Walker, M. J. (2005) Surface analyses and immune reactivities of major cell wall-associated proteins of group $A$ Streptococcus. Infect. Immun. 73, 3137-3146

37. Stevens, D. L., Tanner, M. H., and Winship, J. (1989) Reappearance of scarlet fever toxin A among streptococci in the Rocky Mountain West: severe group A streptococcal infections associated with a toxic shock-like syndrome. N. Engl. J. Med. 321, 1-7

38. Musser, J. M., Hauser, A. R., Kim, M. H., Schlievert, P. M., Nelson, K., and Selander, R. K. (1991) Streptococcus pyogenes causing toxic-shock-like syndrome and other invasive diseases: clonal diversity and pyrogenic exotoxin expression. Proc. Natl. Acad. Sci. U. S. A. 88, 2668-2672

39. Cleary, P. P., Kaplan, E. L., Handley, J. P., Wlazlo, A., Kim, M. H., Hauser, A. R., and Schlievert, P. M. (1992) Clonal basis for resurgence of serious Streptococcus pyogenes disease in the 1980s. Lancet 339, 518-521

40. Bachert, B. A., Choi, S. J., LaSala, P. R., Harper, T. I., McNitt, D. H., Boehm, D. T., Caswell, C. C., Ciborowski, P., Keene, D. R., Flores, A. R., Musser, J. M., Squeglia, F., Marasco, D., Berisio, R., and Lukomski, S. (2016) Unique footprint in the sc/1.3 locus affects adhesion and biofilm formation of the invasive M3-type group A Streptococcus. Front Cell Infect Microbio/ 6, 90

41. Sumby, P., Porcella, S., Madrigal, A., Barbian, K., Virtaneva, K., Ricklefs, S., Sturdevant, D., Graham, M., Vuopio-Varkila, J., Hoe, N., and Musser, J. (2005) Evolutionary origin and emergence of a highly successful clone of serotype M1 group A Streptococcus involved multiple horizontal gene transfer events. J. Infect. Dis. 192, 771-782 
42. Aziz, R., and Kotb, M. (2008) Rise and persistence of global M1T1 clone of Streptococcus pyogenes. Emerg. Infect. Dis. 14, 1511-1517

43. Green, N. M., Beres, S. B., Graviss, E. A., Allison, J. E., McGeer, A. J., Vuopio-Varkila, J., LeFebvre, R. B., and Musser, J. M. (2005) Genetic diversity among type emm28 group A Streptococcus strains causing invasive infections and pharyngitis. J. Clin. Microbiol. 43, 40834091

44. Stevens, D. L., and Kaplan, E. L. (eds). (2000) Streptococcal infections: clinical aspects, microbiology, and molecular pathogenesis, Oxford University Press, New York, N.Y.

45. Chuang, I., Van Beneden, C., Beall, B., and Schuchat, A. (2002) Population-based surveillance for postpartum invasive group a Streptococcus infections, 1995-2000. Clin. Infect. Dis. 35, 665670

46. Gaworzewska, E., and Colman, G. (1988) Changes in the pattern of infection caused by Streptococcus pyogenes. Epidemiol. Infect. 100, 257-269

47. Eriksson, B. K., Norgren, M., McGregor, K., Spratt, B. G., and Normark, B. H. (2003) Group A streptococcal infections in Sweden: a comparative study of invasive and noninvasive infections and analysis of dominant T28 emm28 isolates. Clin. Infect. Dis. 37, 1189-1193

48. Shulman, S. T., Tanz, R. R., Kabat, W., Kabat, K., Cederlund, E., Patel, D., Li, Z., Sakota, V., Dale, J. B., and Beall, B. (2004) Group A streptococcal pharyngitis serotype surveillance in North America, 2000-2002. Clin. Infect. Dis. 39, 325-332

49. O'Brien, K. L., Beall, B., and Barrett, N. L. (2002) Epidemiology of invasive group A Streptococcus disease in the United States, 1995-1999. Clinical and Infectious Diseases 35, 268276

50. Hoe, N., Lukomska, E., Musser, J., and Lukomski, S. (2007) Characterization of the immune response to collagen-like proteins Scl1 and Scl2 of serotype M1 and M28 group A Streptococcus. FEMS Microbiol. Lett. 277, 142-149

51. Chaudhary, P., Kumar, R., Sagar, V., Sarkar, S., Singh, R., Ghosh, S., Singh, S., and Chakraborti, A. (2018) Assessment of Cpa, Scl1 and Scl2 in clinical group A streptococcus isolates and patients from north India: an evaluation of the host pathogen interaction. Res. Microbiol. 169, 11-19

52. Akesson, P., Rasmussen, M., Mascini, E., von Pawel-Rammingen, U., Janulczyk, R., Collin, M., Olsen, A., Mattsson, E., Olsson, M. L., Björck, L., and Christensson, B. (2004) Low antibody levels against cell wall-attached proteins of Streptococcus pyogenes predispose for severe invasive disease. The Journal of Infectious Diseases 189, 797-804

53. Lukomski, S., Bachert, B. A., Squeglia, F., and Berisio, R. (2017) Collagen-like proteins of pathogenic streptococci. Mol. Microbiol. 103, 919-930

54. Xu, Y., Keene, D. R., Bujnicki, J. M., Höök, M., and Lukomski, S. (2002) Streptococcal Scl1 and Scl2 proteins form collagen-like triple helices. J Biol Chem 277, 27312-27318 
55. Mohs, A., Silva, T., Yoshida, T., Amin, R., Lukomski, S., Inouye, M., and Brodsky, B. (2007) Mechanism of stabilization of a bacterial collagen triple helix in the absence of hydroxyproline. $J$ Biol Chem 282, 29757-29765

56. Okuyama, K. (2008) Revisiting the molecular structure of collagen. Connect. Tissue Res. 49, 299310

57. Brodsky, B., and Persikov, A. V. (2005) Molecular structure of the collagen triple helix. Adv. Protein Chem. 70, 301-339

58. Shoulders, M. D., and Raines, R. T. (2009) Collagen structure and stability. Annu. Rev. Biochem. 78, 929-958

59. Berisio, R., Vitagliano, L., Mazzarella, L., and Zagari, A. (2002) Crystal structure of the collagen triple helix model [(Pro-Pro-Gly) $\left.{ }_{10}\right]_{3}$. Protein Sci. 11, 262-270

60. Berisio, R., Granata, V., Vitagliano, L., and Zagari, A. (2004) Imino acids and collagen triple helix stability: characterization of collagen-like polypeptides containing Hyp-Hyp-Gly sequence repeats. J. Am. Chem. Soc. 126, 11402-11403

61. Berisio, R., Granata, V., Vitagliano, L., and Zagari, A. (2004) Characterization of collagen-like heterotrimers: Implications for triple-helix stability. Biopolymers 73, 682-688

62. Vitagliano, L., Berisio, R., Mastrangelo, A., Mazzarella, L., and Zagari, A. (2001) Preferred proline puckerings in cis and trans peptide groups: Implications for collagen stability. Protein Sci: 10, 2627-2632

63. Berisio, R., De Simone, A., Ruggiero, A., Improta, R., and Vitagliano, L. (2009) Role of side chains in collagen triple helix stabilization and partner recognition. J. Pept. Sci. 15, 131-140

64. Improta, R., Berisio, R., and Vitagliano, L. (2008) Contribution of dipole-dipole interactions to the stability of the collagen triple helix. Protein Sci. 17, 955-961

65. Chan, V. C., Ramshaw, J. A., Kirkpatrick, A., Beck, K., and Brodsky, B. (1997) Positional preferences of ionizable residues in Gly-X-Y triplets of the collagen triple-helix. J Biol Chem 272, 31441-31446

66. Leikina, E., Mertts, M. V., Kuznetsova, N., and Leikin, S. (2002) Type I collagen is thermally unstable at body temperature. Proc. Natl. Acad. Sci. U. S. A. 99, 1314-1318

67. Han, R., Zwiefka, A., Caswell, C. C., Xu, Y., Keene, D. R., Lukomska, E., Zhao, Z., Höök, M., and Lukomski, S. (2006) Assessment of prokaryotic collagen-like sequences derived from streptococcal Scl1 and Scl2 proteins as a source of recombinant GXY polymers. Appl. Microbiol. Biotechnol. 72, 109-115

68. Xu, C., Yu, Z., Inouye, M., Brodsky, B., and Mirochnitchenko, O. (2010) Expanding the family of collagen proteins: recombinant bacterial collagens of varying composition form triple-helices of similar stability. Biomacromolecules 11, 348-356 
69. Han, R., Caswell, C. C., Lukomska, E., Keene, D. R., Pawlowski, M., Bujnicki, J. M., Kim, J. K., and Lukomski, S. (2006) Binding of the low-density lipoprotein by streptococcal collagen-like protein Scl1 of Streptococcus pyogenes. Mol. Microbiol. 61, 351-367

70. Squeglia, F., Bachert, B., Romano, M., Lukomski, S., and Berisio, R. (2013) Crystallization and preliminary X-ray crystallographic analysis of the variable domain of Scl2.3, a streptococcal collagen-like protein from invasive M3-type Streptococcus pyogenes. Acta Crystallogr. Sect. F Struct. Biol. Cryst. Commun. 69, 1023-1025

71. Squeglia, F., Bachert, B., De Simone, A., Lukomski, S., and Berisio, R. (2014) The crystal structure of the streptococcal collagen-like protein 2 globular domain from invasive M3-type group A Streptococcus shows significant similarity to immunomodulatory HIV protein gp41. J Biol Chem 289, 5122-5133

72. Yu, Z., Mirochnitchenko, O., Xu, C., Yoshizumi, A., Brodsky, B., and Inouye, M. (2010) Noncollagenous region of the streptococcal collagen-like protein is a trimerization domain that supports refolding of adjacent homologous and heterologous collagenous domains. Protein Sci. 19, $775-785$

73. Humtsoe, J. O., Kim, J. K., Xu, Y., Keene, D. R., Höök, M., Lukomski, S., and Wary, K. K. (2005) A streptococcal collagen-like protein interacts with the $\alpha 2 \beta 1$ integrin and induces intracellular signaling. J. Biol. Chem. 280, 13848-13857

74. Caswell, C. C., Lukomska, E., Seo, N. S., Höök, M., and Lukomski, S. (2007) Scl1-dependent internalization of group A Streptococcus via direct interactions with the $\alpha 2 \beta 1$ integrin enhances pathogen survival and re-emergence. Mol. Microbiol. 64, 1319-1331

75. Caswell, C. C., Barczyk, M., Keene, D. R., Lukomska, E., Gullberg, D. E., and Lukomski, S. (2008) Identification of the first prokaryotic collagen sequence motif that mediates binding to human collagen receptors, integrins $\alpha 2 \beta 1$ and $\alpha 11 \beta 1$. J Biol Chem 283, 36168-36175

76. McNitt, D. H., Choi, S. J., Keene, D. R., Van De Water, L., Squeglia, F., Berisio, R., and Lukomski, S. (2018) Surface-exposed loops and an acidic patch in the Scl1 protein of group A Streptococcus enable Scl1 binding to wound-associated fibronectin. J. Biol. Chem. 293, 77967810

77. Caswell, C. C., Han, R., Hovis, K. M., Ciborowski, P., Keene, D. R., Marconi, R. T., and Lukomski, S. (2008) The Scl1 protein of M6-type group A Streptococcus binds the human complement regulatory protein, factor $\mathrm{H}$, and inhibits the alternative pathway of complement. Mol. Microbiol. 67, 584-596

78. Reuter, M., Caswell, C. C., Lukomski, S., and Zipfel, P. F. (2010) Binding of the human complement regulators CFHR1 and factor $\mathrm{H}$ by streptococcal collagen-like protein 1 (Scl1) via their conserved $\mathrm{C}$ termini allows control of the complement cascade at multiple levels. $J$ Biol Chem 285, 38473-38485 
79. Gao, Y., Liang, C., Zhao, R., Lukomski, S., and Han, R. (2010) The Scl1 of M41-type group A Streptococcus binds the high-density lipoprotein. FEMS Microbiol. Lett. 309, 55-61

80. Påhlman, L. I., Marx, P. F., Mörgelin, M., Lukomski, S., Meijers, J. C., and Herwald, H. (2007) Thrombin-activatable fibrinolysis inhibitor binds to Streptococcus pyogenes by interacting with collagen-like proteins A and B. J Biol Chem 282, 24873-24881

81. Dohrmann, S., Anik, S., Olson, J., Anderson, E. L., Etesami, N., No, H., Snipper, J., Nizet, V., and Okumura, C. Y. (2014) Role for streptococcal collagen-like protein 1 in M1T1 group A Streptococcus resistance to neutrophil extracellular traps. Infect. Immun. 82, 4011-4020

82. Van De Water, L., Varney, S., and Tomasek, J. J. (2013) Mechanoregulation of the myofibroblast in wound contraction, scarring, and fibrosis: opportunities for new therapeutic intervention. Adv Wound Care (New Rochelle) 2, 122-141

83. Gurtner, G. C., Werner, S., Barrandon, Y., and Longaker, M. T. (2008) Wound repair and regeneration. Nature 453, 314-321

84. Clark, R. A., Lanigan, J. M., DellaPelle, P., Manseau, E., Dvorak, H. F., and Colvin, R. B. (1982) Fibronectin and fibrin provide a provisional matrix for epidermal cell migration during wound reepithelialization. J. Invest. Dermatol. 79, 264-269

85. Olczyk, P., Mencner, Ł., and Komosinska-Vassev, K. (2014) The role of the extracellular matrix components in cutaneous wound healing. BioMed Research International 2014, 747584

86. Xue, M., and Jackson, C. J. (2015) Extracellular matrix reorganization during wound healing and its impact on abnormal scarring. Adv. Wound Care 4, 119-136

87. Pankov, R., and Yamada, K. M. (2002) Fibronectin at a glance. J. Cell Sci. 115, 3861-3863

88. Ffrench-Constant, C. (1995) Alternative splicing of fibronectin--many different proteins but few different functions. Exp. Cell Res. 221, 261-271

89. Hynes, R. O. (1990) Fibronectins, Springer-Verlag, New York, NY

90. To, W. S., and Midwood, K. S. (2011) Plasma and cellular fibronectin: distinct and independent functions during tissue repair. Fibrogenesis Tissue Repair 4, 21

91. Ffrench-Constant, C., and Hynes, R. O. (1989) Alternative splicing of fibronectin is temporally and spatially regulated in the chicken embryo. Development 106, 375-388

92. Ffrench-Constant, C., Van De Water, L., Dvorak, H. F., and Hynes, R. O. (1989) Reappearance of an embryonic pattern of fibronectin splicing during wound healing in the adult rat. J. Cell Biol. 109, 903-914

93. Coito, A. J., Brown, L. F., Peters, J. H., Kupiec-Weglinski, J. W., and Van De Water, L. (1997) Expression of fibronectin splicing variants in organ transplantation: a differential pattern between rat cardiac allografts and isografts. Am. J. Pathol. 150, 1757-1772

94. Singh, P., Reimer, C. L., Peters, J. H., Stepp, M. A., Hynes, R. O., and Van De Water, L. (2004) The spatial and temporal expression patterns of integrin $\alpha 9 \beta 1$ and one of its ligands, the EIIIA segment of fibronectin, in cutaneous wound healing. J. Invest. Dermatol. 123, 1176-1181 
95. Muro, A. F., Chauhan, A. K., Gajovic, S., laconcig, A., Porro, F., Stanta, G., and Baralle, F. E. (2003) Regulated splicing of the fibronectin EDA exon is essential for proper skin wound healing and normal lifespan. J. Cell Biol. 162, 149-160

96. Longmate, W. M., Lyons, S. P., Chittur, S. V., Pumiglia, K. M., Van De Water, L., and DiPersio, C. M. (2017) Suppression of integrin $\alpha 3 \beta 1$ by $\alpha 9 \beta 1$ in the epidermis controls the paracrine resolution of wound angiogenesis. J. Cell Biol. 216, 1473-1488

97. Leahy, D. J., Aukhil, I., and Erickson, H. P. (1996) $2.0 \AA$ A crystal structure of a four-domain segment of human fibronectin encompassing the RGD loop and synergy region. Cell 84, 155-164

98. Niimi, T., Osawa, M., Yamaji, N., Yasunaga, K., Sakashita, H., Mase, T., Tanaka, A., and Fujita, S. (2001) NMR structure of human fibronectin EDA. J. Biomol. NMR 21, 281-284

99. Liao, Y. F., Gotwals, P. J., Koteliansky, V. E., Sheppard, D., and Van De Water, L. (2002) The EIIIA segment of fibronectin is a ligand for integrins $\alpha 9 \beta 1$ and $\alpha 4 \beta 1$ providing a novel mechanism for regulating cell adhesion by alternative splicing. J Biol Chem 277, 14467-14474

100. Shinde, A. V., Bystroff, C., Wang, C., Vogelezang, M. G., Vincent, P. A., Hynes, R. O., and Van De Water, L. (2008) Identification of the peptide sequences within the EIIIA (EDA) segment of fibronectin that mediate integrin a9b1-dependent cellular activities. J Biol Chem 283, 2858-2870

101. Kohan, M., Muro, A. F., White, E. S., and Berkman, N. (2010) EDA-containing cellular fibronectin induces fibroblast differentiation through binding to $\alpha 4 \beta 7$ integrin receptor and MAPK/Erk 1/2dependent signaling. FASEB J. 24, 4503-4512

102. White, E. S., and Muro, A. F. (2011) Fibronectin splice variants: understanding their multiple roles in health and disease using engineered mouse models. IUBMB Life 63, 538-546

103. Singh, P., Chen, C., Pal-Ghosh, S., Stepp, M. A., Sheppard, D., and Van De Water, L. (2009) Loss of integrin $\alpha 9 \beta 1$ results in defects in proliferation, causing poor re-epithelialization during cutaneous wound healing. J. Invest. Dermatol. 129, 217-228

104. Shinde, A. V., Kelsh, R., Peters, J. H., Sekiguchi, K., Van De Water, L., and McKeown-Longo, P. J. (2015) The $\alpha 4 \beta 1$ integrin and the EDA domain of fibronectin regulate a profibrotic pheotype in dermal fibroblasts Matrix Biol. 41, 26-35

105. Mitchell, K., Szekeres, C., Milano, V., Svenson, K. B., Nilsen-Hamilton, M., Kreidberg, J. A., and DiPersio, C. M. (2009) $\alpha 3 \beta 1$ integrin in epidermis promotes wound angiogenesis and keratinocyte-to-endothelial-cell crosstalk through the induction of MRP3. J. Cell Sci. 122, 17781787

106. Longmate, W. M., Monichan, R., Chu, M.-L., Tsuda, T., Mahoney, M. G., and DiPersio, C. M. (2014) Reduced fibulin-2 contributes to loss of basement membrane integrity and skin blistering in mice lacking integrin $\alpha 3 \beta 1$ in the epidermis. J. Invest. Dermatol. 134, 1609-1617

107. Julier, Z., Martino, M. M., de Titta, A., Jeanbart, L., and Hubbell, J. A. (2015) The TLR4 agonist fibronectin extra domain A is cryptic, exposed by elastase-2; use in a fibrin matrix cancer vaccine. Sci. Rep. 5, 8569 
108. Okamura, Y., Watari, M., Jerud, E. S., Young, D. W., Ishizaka, S. T., Rose, J., Chow, J. C., and Strauss, J. F., 3rd. (2001) The extra domain A of fibronectin activates Toll-like receptor 4. J Biol Chem 276, 10229-10233

109. Kelsh, R., You, R., Horzempa, C., Zheng, M., and McKeown-Longo, P. J. (2014) Regulation of the innate immune response by fibronectin: synergism between the III-1 and EDA domains. PLoS One 9, e102974

110. Ozeri, V., Rosenshine, I., Mosher, D. F., Fässler, R., and Hanski, E. (1998) Roles of integrins and fibronectin in the entry of Streptococcus pyogenes into cells via protein F1. Mol. Microbiol. 30, 625-637

111. Joh, D., Speziale, P., Gurusiddappa, S., Manor, J., and Höök, M. (1998) Multiple specificities of the staphylococcal and streptococcal fibronectin-binding microbial surface components recognizing adhesive matrix molecules. Eur. J. Biochem. 258, 897-905

112. Schwarz-Linek, U., Werner, J. M., Pickford, A. R., Gurusiddappa, S., Kim, J. H., Pilka, E. S., Briggs, J. A., Gough, T. S., Höök, M., Campbell, I. D., and Potts, J. R. (2003) Pathogenic bacteria attach to human fibronectin through a tandem beta-zipper. Nature 423, 177-181

113. Yamaguchi, M., Terao, Y., and Kawabata, S. (2013) Pleiotropic virulence factor - Streptococcus pyogenes fibronectin-binding proteins. Cell. Microbiol. 15, 503-511

114. Hayes, C. S., and Williamson, H., Jr. (2001) Management of group A beta-hemolytic streptococcal pharyngitis. Am. Fam. Physician 63, 1557-1564

115. Neeman, R., Keller, N., Barzilai, A., Korenman, Z., and Sela, S. (1998) Prevalence of internalisation-associated gene, prtF1, among persisting group-A Streptococcus strains isolated from asymptomatic carriers. Lancet 352, 1974-1977

116. Stjernquist-Desatnik, A., Orrling, A., Schalén, C., and Kamme, C. (1992) Penicillin tolerance in group A streptococci and treatment failure in streptococcal tonsillitis. Acta Otolaryngol. 492, 68-71

117. Pichichero, M. E., Casey, J. R., Mayes, T., Francis, A. B., Marsocci, S. M., Murphy, A. M., and Hoeger, W. (2000) Penicillin failure in streptococcal tonsillopharyngitis: causes and remedies. Pediatr. Infect. Dis. J. 19, 917-923

118. Martin , J. M., Green , M., Barbadora , K. A., and Wald, E. R. (2002) Erythromycin-resistant group A streptococci in schoolchildren in Pittsburgh. N. Engl. J. Med. 346, 1200-1206

119. Perez-Trallero, E., Montes, M., Orden, B., Tamayo, E., Garcia-Arenzana, J., and Marimon, J. (2007) Phenotypic and genotypic characterization of Streptococcus pyogenes displaying the MLSB-phenotype of macrolide resistance in Spain: 1999-2005. Antimicrob. Agents Chemother.

120. Lancefield, R. C. (1959) Persistence of type-specific antibodies in man following infection with group A streptococci. J. Exp. Med. 110, 271-292

121. Cunningham, M. W. (2000) Cross-reactive antigens of group A streptococci. in Gram-positive pathogens (Fischetti, V. A., Novick, R. P., Ferretti, J. J., Portnoy, D. A., and Rood, J. I. eds.), American Society for Microbiology Press, Washington, D.C. pp 66-77 
122. Dale, J. B. (2008) Current status of group A streptococcal vaccine development. Adv. Exp. Med. Biol. 609, 53-63

123. Steer, A. C., Carapetis, J. R., Dale, J. B., Fraser, J. D., Good, M. F., Guilherme, L., Moreland, N. J., Mulholland, E. K., Schodel, F., and Smeesters, P. R. (2016) Status of research and development of vaccines for Streptococcus pyogenes. Vaccine 34, 2953-2958

124. Steer, A. C., Law, I., Matatolu, L., Beall, B. W., and Carapetis, J. R. (2009) Global emm type distribution of group A streptococci: systematic review and implications for vaccine development. The Lancet Infectious Diseases 9, 611-616

125. Guirguis, N., Fraser, D. W., Facklam, R. R., El Kholy, A., and Wannamaker, L. W. (1982) Typespecific immunity and pharyngeal acquisition of group A Streptococcus. Am. J. Epidemiol. 116, 933-939

126. Peng, Y. Y., Yoshizumi, A., Danon, S. J., Glattauer, V., Prokopenko, O., Mirochnitchenko, O., Yu, Z., Inouye, M., Werkmeister, J. A., Brodsky, B., and Ramshaw, J. A. (2010) A Streptococcus pyogenes derived collagen-like protein as a non-cytotoxic and non-immunogenic cross-linkable biomaterial. Biomaterials 31, 2755-2761

127. Bhardwaj, G., Mulligan, V. K., Bahl, C. D., Gilmore, J. M., Harvey, P. J., Cheneval, O., Buchko, G. W., Pulavarti, S. V. S. R. K., Kaas, Q., Eletsky, A., Huang, P.-S., Johnsen, W. A., Greisen, P., Jr., Rocklin, G. J., Song, Y., Linsky, T. W., Watkins, A., Rettie, S. A., Xu, X., Carter, L. P., Bonneau, R., Olson, J. M., Coutsias, E., Correnti, C. E., Szyperski, T., Craik, D. J., and Baker, D. (2016) Accurate de novo design of hyperstable constrained peptides. Nature 538, 329

128. Gaillard, V., Galloux, M., Garcin, D., Eléouët, J.-F., Le Goffic, R., Larcher, T., Rameix-Welti, M.A., Boukadiri, A., Héritier, J., Segura, J.-M., Baechler, E., Arrell, M., Mottet-Osman, G., and Nyanguile, O. (2017) A short double-stapled peptide inhibits respiratory syncytial virus entry and spreading. Antimicrob. Agents Chemother. 61

129. Wiedmann, M. M., Tan, Y. S., Wu, Y., Aibara, S., Xu, W., Sore, H. F., Verma, C. S., Itzhaki, L., Stewart, M., Brenton, J. D., and Spring, D. R. (2017) Development of cell-permeable, non-helical constrained peptides to target a key protein-protein interaction in ovarian cancer. Angewandte Chemie International Edition 56, 524-529

130. Nizet, V., Ohtake, T., Lauth, X., Trowbridge, J., Rudisill, J., Dorschner, R. A., Pestonjamasp, V., Piraino, J., Huttner, K., and Gallo, R. L. (2001) Innate antimicrobial peptide protects the skin from invasive bacterial infection. Nature 414, 454-457

131. Dorschner, R. A., Pestonjamasp, V. K., Tamakuwala, S., Ohtake, T., Rudisill, J., Nizet, V., Agerberth, B., Gudmundsson, G. H., and Gallo, R. L. (2001) Cutaneous injury induces the release of cathelicidin anti-microbial peptides active against group A Streptococcus. J. Invest. Dermatol. 117, 91-97 


\section{FIGURES}

\section{Figure 1}

A
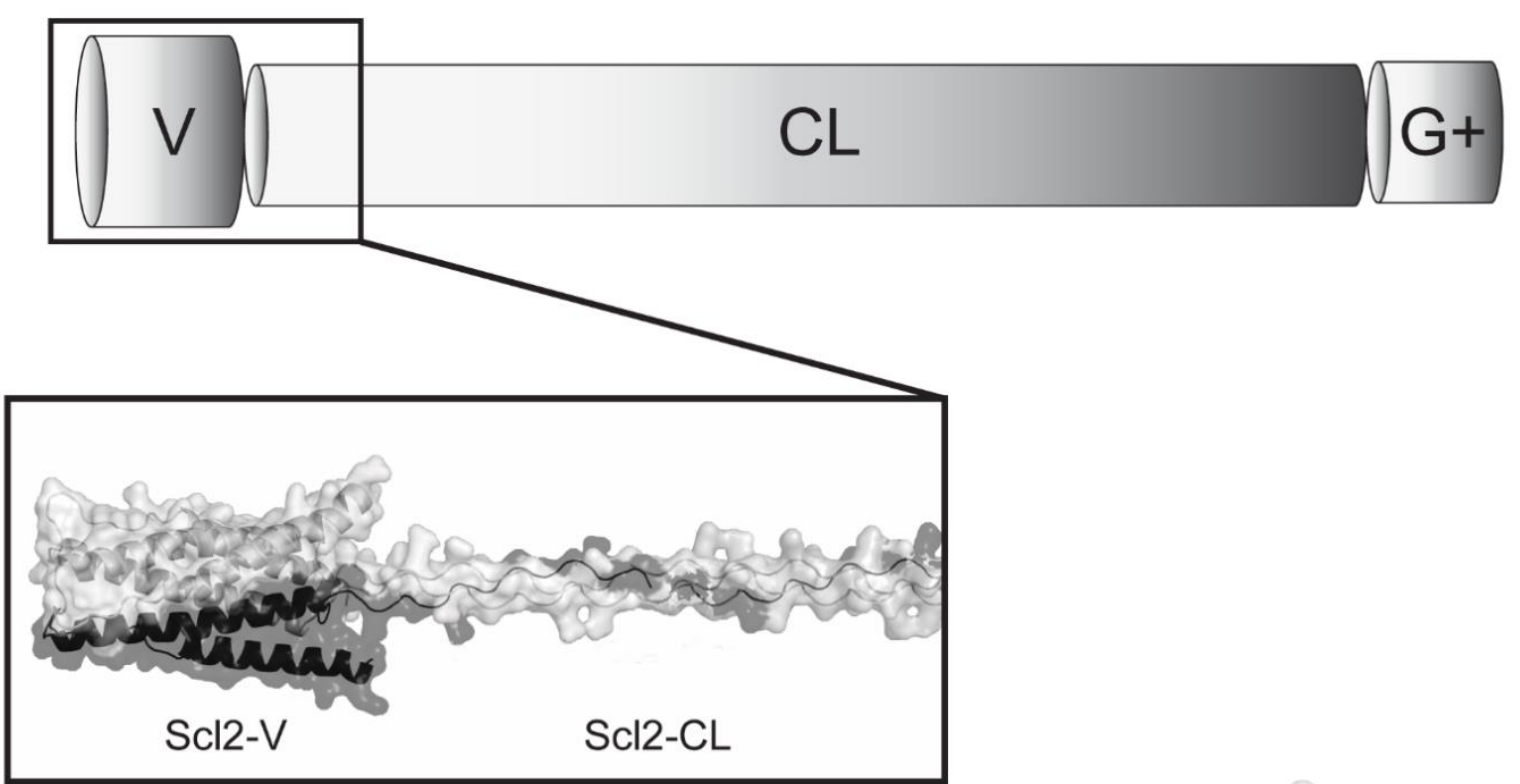

B
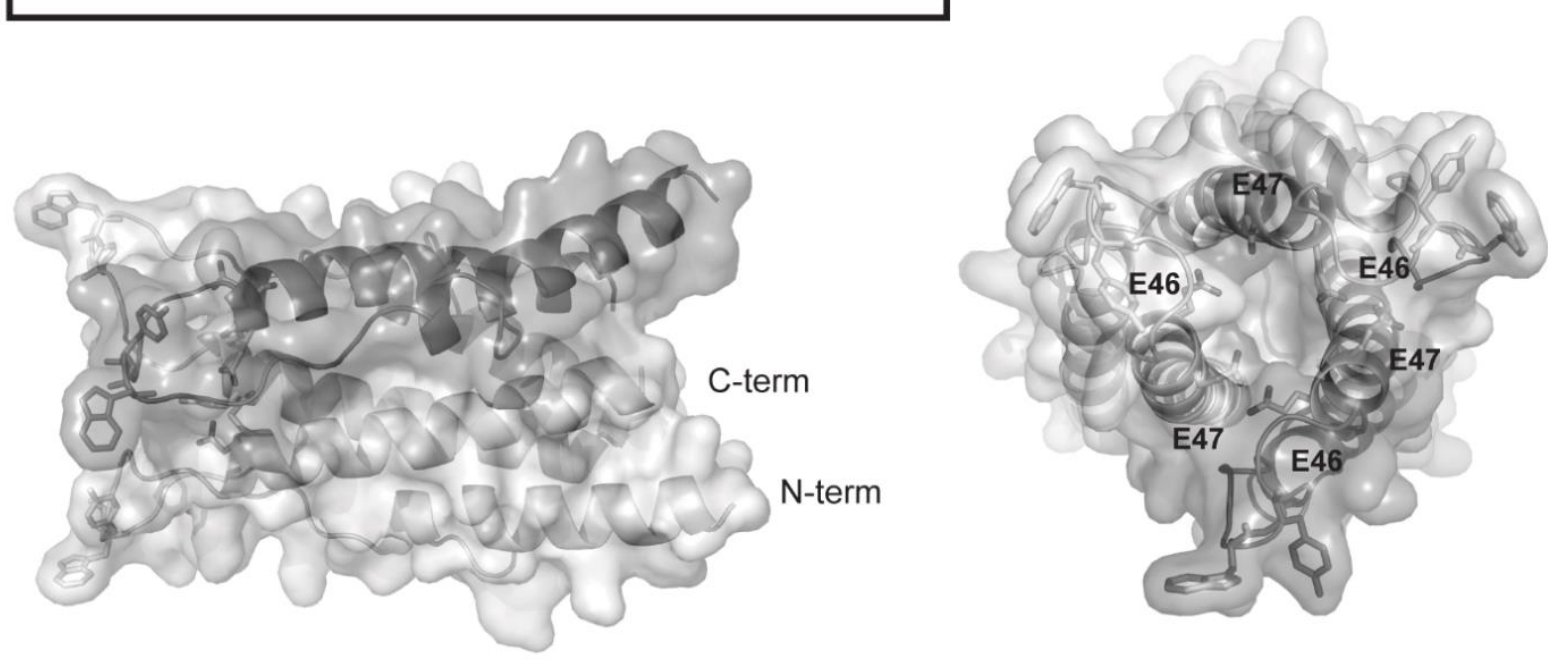

Figure 1: Scl1 and Scl2 structure. (A) Top, Schematic representation of the common domain organization of Scl1 and Scl2 proteins. Domains: V, the N-terminal globular sequence-variable domain; $\mathrm{CL}$, the collagenlike domain; $\mathrm{G}_{+}$, the C-terminal cell-wall-anchor domain. Bottom insert, Cartoon representation of Scl2-V$\mathrm{CL}$ structure. This structure has been obtained by combining the crystal structure of the Scl2-V domain, with the modeled structure of the CL domain, generated using the structure of the collagen peptide (ProPro-Gly) 10 as a template; only the first 15 Gly-X-Y repeats is shown for clarity. (B), Homology model of the Scl1-V domain. Left, Side view of the Scl1-V domain, showing the six-helix bundle fold, with three pairs of anti-parallel $\alpha$-helices and connecting loop segments. The $\mathrm{N}$-terminus and C-terminus of a single 
representative $\alpha$-helix pair is marked. Right, Top-down view of the Scl1-V domain. The three helix-pairs are shown, wrapped in a left-handed orientation. Negatively-charged amino acids, and their positions within the Scl1-V domain, are marked. These residues are conserved in EDA-binding-positive Scl1 proteins. The homology model is based on the crystal structure of the Scl2-V domain originating from M3-type group A Streptococcus (PDB accession code: $4 \mathrm{nsm}$ ). 


\section{Figure 2}

A

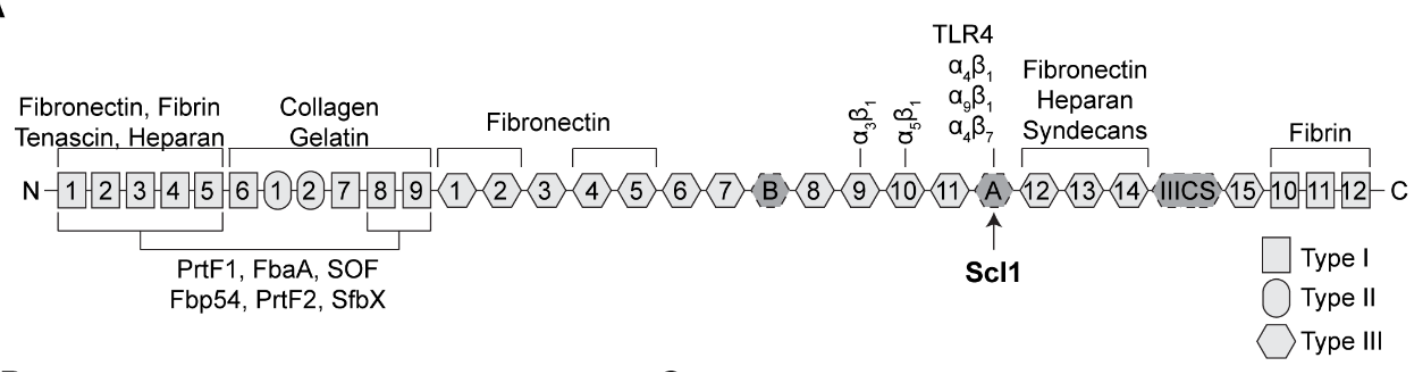

B

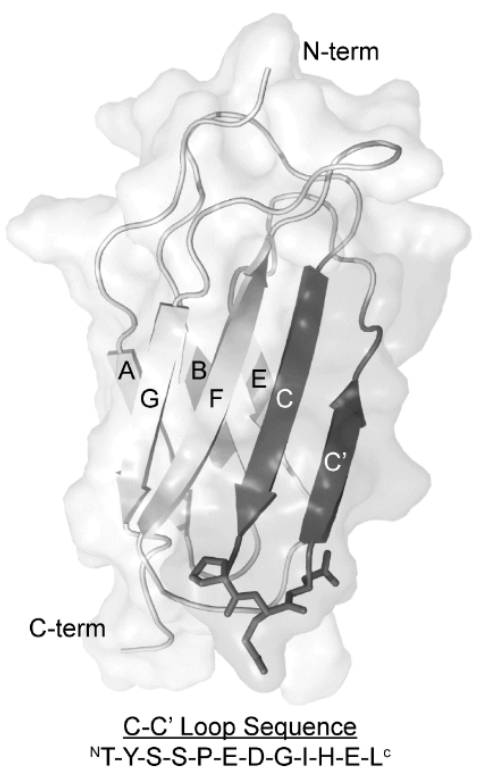

C

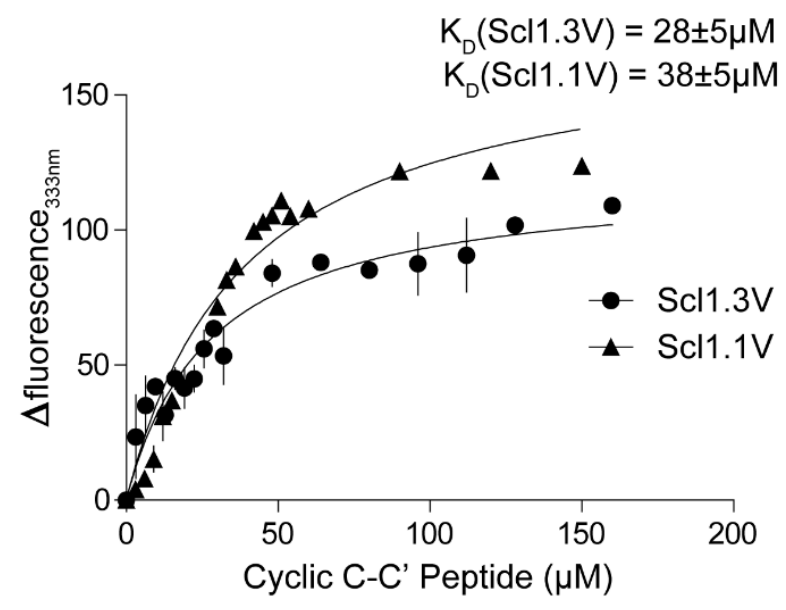

Figure 2: Scl1 binds to cellular fibronectin that contains extra domain A. Schematic of fibronectin, model of EDA, and fluorescence binding data between C-C' loop peptide and recombinant Scl1 (rScl1) proteins. ( $A$ ) Schematic of fibronectin, with fibronectin type I, II, and III repeat domains denoted by squares, ovals, and hexagons, respectively. Regular Fn domains are shown in light grey and are numbered, constitutively, whereas alternatively spliced extra domains A, B and IIICS are shown in dark gray. Scl1EDA binding is marked with an arrow and binding regions for several GAS Fn-binding proteins (PrtF1, FbaA, SOF, etc.) are also indicated. (B) Cartoon representation of the EDA domain of fibronectin. Model (PDB accession code: $1 \mathrm{~J} 8 \mathrm{~K}$ ) shows the $\beta$-sandwich structure, with each $\beta$-strand marked $\left(A, B, C, C^{\prime}, D\right.$, $E, F)$; the $C$-C' loop is shown in ball-and-stick style and the corresponding amino acid sequence is shown below. $(C)$ Binding assay based on tryptophan fluorescence for the Scl1-EDA interaction. Binding was assessed between a synthetic C-C' loop-derived cyclic peptide and recombinant Scl1 forms derived from M1 (Scl1.1V) and M3-type (Scl1.3V) GAS. Tryptophan fluorescence quenching analysis shows the doseresponse curve of the fluorescence values for both Scl1.1V and Scl1.3V at $333 \mathrm{~nm}$ versus the concentration of C-C' cyclic peptide. 


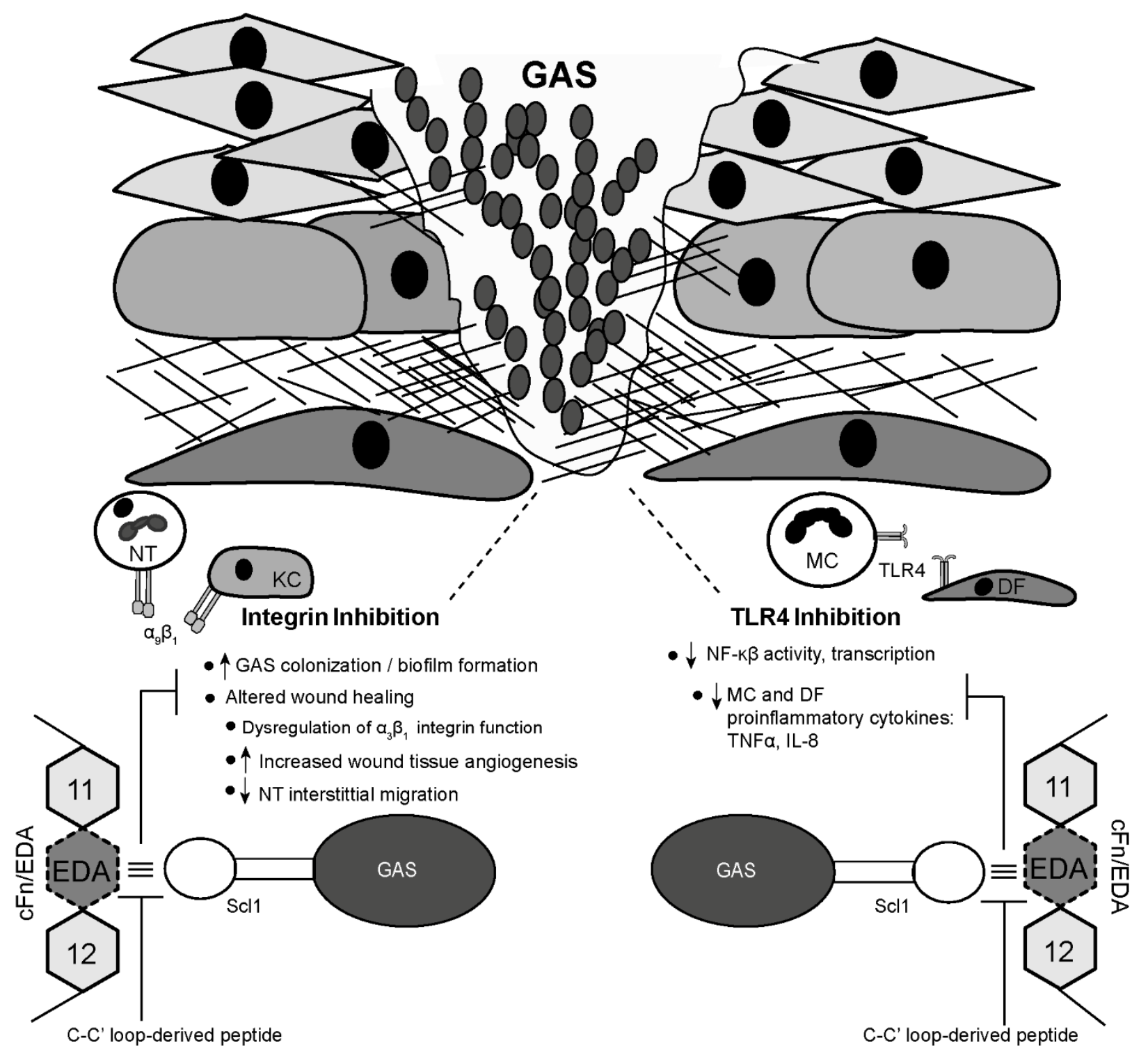

Figure 3: Modulation of host responses by GAS Scl1 during wound colonization. In this model, GAS gains access to the host via the portal of entry, shown here as a breach in protective epidermis (i.e. skin and throat mucosa). The wound microenvironment ECM is enriched with EDA/cFn isoforms. The globular $V$ domain of Scl1 binds to the C-C' loop of EDA, facilitating GAS biofilm formation and wound colonization. Neutrophils $(N T)$ and keratinocytes $(K C)$ bind to the C-C' loop of EDA via integrin receptor $\alpha_{9} \beta_{1}$, regulating important wound-healing functions. EDA/cFn also activates TLR4 on monocytes (MC) and dermal fibroblasts $(D F)$ to stimulate the production of pro-inflammatory cytokines, TNFa and IL-8, through the NFKB signaling cascade. The Scl1-EDA/cFn interaction may alter several important wound-healing functions. Designed C-C' loop-derived peptides could prevent GAS wound colonization and reverse potentially adverse effects of the Scl1-EDA/cFn interaction. 


\title{
APPENDIX 3: ADDITIONAL PUBLISHED DATA UNIQUE FOOTPRINT IN THE SCL1.3 LOCUS AFFECTS ADHESION AND BIOFILM FORMATION OF THE INVASIVE M3-TYPE GROUP A STREPTOCOCCUS
}

\author{
Beth A. Bachert, Soo Jeon Choi, Paul R. LaSala, Tiffany Harper, Dudley H. McNitt, \\ Dylan T. Boehm, Clayton C. Caswell, Pawel Ciborowski, Douglas R. Keene, Anthony R. \\ Flores, James M. Musser, Flavia Squeglia, Daniela Marasco, Rita Berisio, and Slawomir \\ Lukomski \\ Published in Frontiers in Cellular and Infection Microbiology 2016 6:90

\begin{abstract}
(Publication demonstrated that the lack of surface expression of Scl1 from M3 serotype of group $A$ Streptococcus contributes to the invasiveness of this $M$ type. I constructed and assisted in the construction of two plasmids used in this study - pSI503, pSL514)
\end{abstract}

\begin{abstract}
The streptococcal collagen-like proteins 1 and 2 ( $\mathrm{Scl} 1$ and $\mathrm{Scl} 2$ ) are major surface adhesins that are ubiquitous among group A Streptococcus (GAS). Invasive M3-type strains, however, have evolved two unique conserved features in the scl1 locus: (i) an IS1548 element insertion in the scl1 promoter region and (ii) a nonsense mutation within the scl1 coding sequence. The scl1 transcript is drastically reduced in M3-type GAS, contrasting with a high transcription level of scl1 allele in invasive M1-type GAS. This leads to a lack of Scl1 expression in M3 strains. In contrast, while scl2 transcription and Scl2 production are elevated in M3 strains, M1 GAS lack Scl2 surface expression. M3type strains were shown to have reduced biofilm formation on inanimate surfaces coated with cellular fibronectin and laminin, and in human skin equivalents. Repair of the nonsense mutation and restoration of Scl1 expression on M3-GAS cells, restores biofilm formation on cellular fibronectin and laminin coatings. Inactivation of sc/1 in biofilmcapable M28 and M41 strains results in larger skin lesions in a mouse model, indicating that lack of Scl1 adhesin promotes bacterial spread over localized infection. These studies suggest the uniquely evolved sc/1 locus in the M3-type strains, which prevents surface expression of the major Scl1 adhesin, contributed to the emergence of the invasive M3-type strains. Furthermore these studies provide insight into the molecular
\end{abstract}


mechanisms mediating colonization, biofilm formation, and pathogenesis of group A streptococci.

\section{INTRODUCTION}

Group A Streptococcus (GAS) or Streptococcus pyogenes is a human-specific Grampositive pathogen responsible for significant morbidity and mortality worldwide $(1,2)$. The clinical outcomes resulting from GAS infection range from superficial pharyngitis and impetigo to severe life-threatening diseases, such as streptococcal toxic shock syndrome and necrotizing fasciitis, as well as post-infectious sequelae, including rheumatic fever, rheumatic heart disease, and post-streptococcal glomerulonephritis (3). Historically, GAS has been a significant cause of puerperal sepsis, scarlet fever, erysipelas, and pharyngitis (4). GAS strains are epidemiologically subtyped based on nucleotide sequence variation at the 5'-end of the emm gene, reflecting differences in the hypervariable $\mathrm{N}$-terminal region of the anti-phagocytic surface protein M. Over $240 \mathrm{M}$-types have been identified (http://www.cdc.gov/abcs/index.html), and certain M-types have been shown to have nonrandom associations with specific disease outcomes (3,5). Since the 1980's there has been resurgence in invasive GAS diseases in the U.S. and other parts of the world. Numerous epidemiology studies conducted in the U.S. (6-11), Canada (12-15), and Europe (16-19) have found associations between infections with M1- and M3-type strains and invasive diseases. Specifically, M3-type strains have been associated with severe invasive disease $(6,19)$ and fatal outcomes $(14,16,17)$.

These epidemiological observations have spurred significant whole genome sequencing efforts aimed at identification of the underlying genetic basis for virulence in M3-type GAS. The complete annotated genomes of invasive M3 strains MGAS315 and SSI-1 have been reported $(20,21)$. These studies have revealed that differing phage elements, insertion sequences, and the large-scale chromosomal inversion identified in SSI-1, contributed to much of the genetic variation between M3 and other M-types. Acquisition of prophages and SNP's drive the expansion of different M3 subclones during epidemic waves of infection $(22,23)$. Additionally, strains causing pharyngitis and those causing invasive infections are derived from the same pool of M3 strains (24). In addition, 
genetic variation in virulence regulators, including RopB and CovRS, which are "hotspots" for the accumulation of mutations, also contributes to the hypervirulence of M3 strains (23-28). The cause of the hyper-invasive phenotype of M3 strains is multifactorial and involves multiple virulence factors controlled by complex regulatory networks that continually undergo remodeling.

The streptococcal collagen-like proteins 1 and 2, or Scl1 and Scl2 (also known as ScIA and ScIB), are major GAS surface adhesins known to contribute to pathogenesis. Both $\mathrm{Scl}$ proteins contain an $\mathrm{N}$-terminal variable region, followed by a collagen-like region containing Gly-X-Y repeats and a cell-wall-anchored C-terminal region (29-33). Transcription of $s c / 1$ is positively regulated by the multiple gene regulator Mga $(30,32,34)$. Scl1 binds cellular fibronectin and laminin (35), and contributes to the formation of biofilm by strains of multiple M-types $(36,37)$. GAS adherence and biofilm formation is enhanced on extracellular matrix (ECM) coatings and on fibroblast-deposited ECM network $(37,38)$, supporting a role for Scl1 in tissue-microcolony formation described during GAS skin infection (39). While the $s c / 1$ gene has been found in every GAS strain tested $(29,32)$, the scl1.3-allele in M3-type strains harbors a null mutation within the coding sequence (29). A rare natural reversion of this polymorphism was identified in a small subset of $M 3$ carrier strains (40).

Scl2-protein expression is regulated during translation by varying numbers of short CAAAA repeats downstream of the start codon that determine whether the protein-coding sequence is in-frame or translation will be prematurely terminated $(30,31)$; genome sequencing indicates majority of the M3-type strain contain in-frame scl2.3 alleles $(18,22)$. Scl2 has been shown in some strains to bind the human thrombin-activatable fibrinolysis inhibitor (41) and contribute to adherence to fibroblasts (31), although its role in GAS pathogenesis is less understood. Recently, the crystal structure of the Scl2.3 globular domain, which is structurally conserved between Scl1 and Scl2, has been reported $(42,43)$ providing insight into the potential binding interactions with host ligands.

In this study, we show that invasive M3-type GAS harbor two unique conserved features of the sc/1 locus including the IS1548 insertion in the promoter region and the null mutation in the coding sequence, which results in a secreted instead of cell-attached Scl1 protein. We demonstrate significantly different expression patterns of $s c / 1$ and scl2 
in M3-type GAS compared to representative strains of M1-, M28- and M41-types. We demonstrate that the expression of the Scl1 adhesin is deficient in serotype M3 strains, as opposed to M1-, M28-, and M41-type strains. However, the Scl2 protein is upregulated in M3 strains compared to M1, M28, and M41 GAS. The M3 strains lacked significant biofilms on cellular fibronectin and laminin coatings, compared to M41-type GAS, and did not form tissue microcolonies in a wounded pseudo-organ skin equivalent model of infection. Recombinant Scl1.3 specifically bound cellular fibronectin and laminin, and restoration of surface expression of Scl1.3 conferred significant biofilm formation by M3 strains. Inactivation of Scl1 expression in biofilm-capable M28- and M41-type GAS resulted in larger skin lesions produced by the mutants in a mouse model of subcutaneous infection, supporting a role for Scl1 in maintaining a localized infection. Our model advocates that the lack of surface-associated Scl1 adhesin in M3-type strains causes decreased tissue adherence and decreased capacity for stable microcolony formation, thus, promoting bacterial spread over localized nidus of infection.

\section{EXPERIMENTAL PROCEDURES}

1. Bacterial strains and growth - MGAS315 and MGAS10870 are fully sequenced invasive M3-type strains $(20,23)$. MGAS315 was isolated from a patient with streptococcal toxic syndrome in the 1980's (6) and MGAS10870 was isolated from a patient with soft tissue infection in Ontario in 2002 (23). Additional strains from epidemiologically diverse M-types were used for comparison: MGAS6183 (M41), MGAS5005 (M1), and MGAS6143

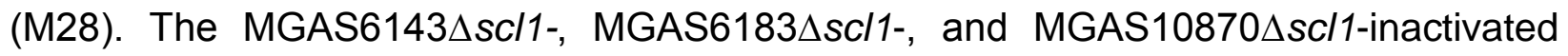
mutant strains have been previously described $(40,44,45)$. Group A Streptococcus cultures were grown in Todd-Hewitt broth (Becton Dickinson and Co.) supplemented with $0.2 \%$ yeast extract (THY medium) and on Brain Heart Infusion agar (Becton Dickinson and Co.) at $37^{\circ} \mathrm{C}$ in an atmosphere with $5 \% \mathrm{CO}_{2}$. For antibiotic selection, erythromycin (5 $\left.\mu \mathrm{g} \mathrm{mL}^{-1}\right)$, chloramphenicol $\left(10 \mu \mathrm{g} \mathrm{mL}^{-1}\right)$, kanamycin $\left(200 \mu \mathrm{g} \mathrm{mL}^{-1}\right)$, and spectinomycin $\left(100 \mu \mathrm{g} \mathrm{mL}^{-1}\right)$ were added to the medium.

Cloning experiments were performed in XL-1 Blue and TB1 E. coli cells, while protein expression experiments were performed in BL21 and TB1 E. coli cells grown in Luria-Bertani media (Difco Laboratories) at $37^{\circ} \mathrm{C}$. For antibiotic selection, 
chloramphenicol $\left(10 \mu \mathrm{g} \mathrm{mL}^{-1}\right)$, kanamycin $\left(50 \mu \mathrm{g} \mathrm{mL}^{-1}\right)$, spectinomycin $\left(100 \mu \mathrm{g} \mathrm{mL}^{-1}\right)$, and ampicillin $\left(100 \mu \mathrm{g} \mathrm{mL}^{-1}\right)$ were added to the medium.

\section{PCR assays}

\subsection{Analytical PCR}

2.1.1. Detection of IS element upstream of scl1 in GAS strains - The presence of IS1548 upstream of scl1 was determined by PCR with primers IS1548F and Scl1R (Figure 1) using Qiagen Taq DNA polymerase (Qiagen, Germantown, MD) as follows: $95^{\circ} \mathrm{C}, 5 \mathrm{~min}-\left[95^{\circ} \mathrm{C} 1 \mathrm{~min}, 62^{\circ} \mathrm{C} 1 \mathrm{~min}, 72^{\circ} \mathrm{C} 1 \mathrm{~min}\right] \times 30$ cycles- $72^{\circ} \mathrm{C}, 10 \mathrm{~min}$. Sequences of primers used in all PCR assays are listed in Table S1.

2.1.2. PCR amplification of $s c / 1.3$ and $s c / 2.3$ alleles - PCR was performed on genomic DNA isolated from M3 strains with primer pairs $232 \mathrm{Up} / 232 \mathrm{Rev}$ for sc/1.3 amplification and sequencing, and Scl2.3 F/R and SclUp/ SclRev for length polymorphism analysis and sequencing of scl2.3, respectively. Amplification was performed using Qiagen Taq DNA polymerase as follows: $s c / 1.3: 95^{\circ} \mathrm{C}, 5 \mathrm{~min}-\left[95^{\circ} \mathrm{C} 1 \mathrm{~min}, 55^{\circ} \mathrm{C} 1 \mathrm{~min}, 72^{\circ} \mathrm{C} 1 \mathrm{~min}\right] \times 30$ cycles- $72^{\circ} \mathrm{C}, 10 \mathrm{~min}$; scl2.3: $95^{\circ} \mathrm{C}, 5 \mathrm{~min}-\left[95^{\circ} \mathrm{C} 1 \mathrm{~min}, 51^{\circ} \mathrm{C} 1 \mathrm{~min}, 72^{\circ} \mathrm{C} 1 \mathrm{~min} 45 \mathrm{sec}\right]$ x30 cycles- $72^{\circ} \mathrm{C}, 10 \mathrm{~min}$. All PCR products were analyzed in a $1 \%$ agarose gel with $1 \mathrm{~kb}$ Plus DNA ladder as a molecular size marker (Life Technologies, Grand Island, NY).

\subsection{Quantitative reverse transcription PCR (qRT-PCR)}

2.2.1. RNA isolation - Total RNA was isolated from the logarithmic phase ( $\left.O D_{600} 0.5\right)$ GAS cultures using RNeasy Protect Bacteria Mini kit (Qiagen), employing an additional pretreatment step with $250 \mu \mathrm{g}$ lysozyme, $100 \mu \mathrm{g}$ proteinase $\mathrm{K}$, and $12.5 \mathrm{U}$ mutanolysin per sample to augment cell wall disruption. Trace genomic DNA was removed by incubation with TurboDNase enzyme (Ambion). RNA quality was assessed in $1 \%$ agarose gel and spectrophotometrically; A260/280 and A260/230 ratios of $>1.8$ were considered 
acceptable. RNA was used immediately in cDNA synthesis or stored at $-80^{\circ} \mathrm{C}$ for no more than 1 week.

2.2.2. Determination of transcription by $\mathbf{q R T}-\mathbf{P C R}$ - Synthesis of cDNA was performed using iScript Select cDNA synthesis kit (Bio-Rad) with $1 \mu \mathrm{g}$ of RNA per reaction and random primer mix. For each sample, a no reverse transcriptase control was performed, containing only RNA, reaction buffer, and random primer mix, to ensure the absence of genomic DNA. Incubations were carried out on a Bio-Rad C1000 Touch Thermal cycler: $25^{\circ} \mathrm{C} 5 \mathrm{~min}, 42^{\circ} \mathrm{C} 30 \mathrm{~min}, 85^{\circ} \mathrm{C} 5 \mathrm{~min}$, and cDNA was stored at $-20^{\circ} \mathrm{C}$ until used in qRTPCR. qRT-PCR reactions were performed with $250 \mathrm{nM}$ primers using SsoAdvanced SYBR Green Universal Supermix (Bio-Rad). For each qRT-PCR reaction, template cDNA, diluted 1:20, control genomic DNA, or no reverse transcriptase control were used. PCR was performed using the following conditions on a Bio-Rad CFX96 Thermal cycler: $95^{\circ} \mathrm{C} 2$ min- $\left[95^{\circ} \mathrm{C} 5 \mathrm{sec}, 60^{\circ} \mathrm{C} 30 \mathrm{sec}\right] \times 30-\left[65^{\circ} \mathrm{C}-95^{\circ} \mathrm{C}, 5 \mathrm{sec}, 0.5^{\circ} \mathrm{C} /\right.$ step]. Standard curves were generated for each primer set using cDNA from MGAS315 to determine the linear range and estimate reaction efficiency. Gene expression of $s c / 1, s c l 2, e m m$, and mga was normalized against the expression of tufA gene, which has previously been identified and validated as an appropriate endogenous control (46). The $\Delta \Delta \mathrm{Ct}$ method was utilized to compare gene expression between MGAS315 and other M3 strains, as well as M1, M28, and M41-type strains. Data was averaged from three independent experiments, each containing three technical replicates. Statistical significance was determined using an unpaired $t$-test.

\section{Recombinant Scl (rScl) proteins}

3.1. Cloning, expression, and purification of M3-derived $\mathrm{rScl}$ proteins - The $\mathrm{rScl}$ proteins were generated using the Strep-tag II cloning, expression and purification system (IBA-GmbH, Gottingen, Germany). Proteins were expressed with a C-terminal affinity tag and purified on Strep-Tactin sepharose, as described (47). rScl proteins rScl1.3V and rScl1.3FL are derived from MGAS315, whereas rScl2.3 protein is derived from the M3 strain MGAS3375. The recombinant Scl2.3V protein has been described previously (42). 
Construct containing the recombinant full-length Scl1.3FL ( $\mathrm{rScl} 1.3 \mathrm{FL}$ ) protein was previously described (40). Briefly, the scl1.3 coding region from MGAS315 was cloned into the E. coli/GAS shuttle vector pJRS525 (48), generating plasmid pSL501. PCR mutagenesis was used to convert the internal TAA stop codon into a GAA glutamate codon, resulting in plasmid pSL502 with continuous full-length sc/1.3FL allele. This sequence was subsequently cloned into the pASK-IBA2 expression vector for recombinant Scl1.3FL protein production; each clone was verified by sequencing. Protein expression constructs are listed in Table 1.

Protein expression was induced by the addition of anhydrotetracycline at $0.2 \mu \mathrm{g}$ $\mathrm{mL}^{-1}$ for 3 hours. Cells were centrifuged and resuspended in high sucrose buffer $(100 \mathrm{mM}$ Tris-HCl, $1 \mathrm{mM}$ EDTA, pH 8.0, $500 \mathrm{mM}$ sucrose) or Cell Lytic B Buffer (Sigma), for separation of the periplasmic fraction and subsequent affinity purification. Proteins were dialyzed against $25 \mathrm{mM}$ HEPES, pH 8.0, and analyzed by SDS-PAGE stained with RAPIDstain $^{\mathrm{TM}}$ (G-Biosciences). Protein sequence was confirmed using mass spectrometry (University of Nebraska Medical Center) and N-terminal Edman Degradation sequencing (lowa State University).

3.2. Electron microscopy of rotary shadowed rScl proteins - Electron microscopy visualization of the rotary shadowed preparations of $\mathrm{rScl} 1.3 \mathrm{FL}$ and $\mathrm{rScl} 2.3$ was used to assess the domain organization of $\mathrm{rScl}$ proteins, as employed previously (49). The $\mathrm{rScl}$ proteins were dialyzed against $0.1 \mathrm{M}$ ammonium bicarbonate and mixed with glycerol to a final concentration of $70 \%$ (vol:vol). Samples were nebulized onto mica chips with an airbrush and rotary-shadowed with carbon/platinum using an electron beam gun. Photomicrograph images were acquired using a transmission electron microscope FEI G2 operated at $80 \mathrm{KV}$.

3.3. Mass spectrometry analysis - Samples from in-gel trypsin digested proteins were cleaned using a Millipore $\mu \mathrm{C} 18$ ZipTip, then resuspended in $0.1 \%$ formic acid. Samples were fractionated on a Eksigent cHiPLC column $(75 \mu \mathrm{m} \times 15 \mathrm{~cm}$ ChromXP C18-CL $3 \mu \mathrm{m}$ $120 \AA$ ) , and resulting peptides were sequenced using 5600 TripleTOF (typical gradient 2$60 \%$ ACN in 60 minutes). Peptides identified were searched against the NCBI protein 
database with Protein Pilot software employing the following settings: search effort, thorough; taxonomy, none. Positive identification was considered as the identification of two or more unique peptides at high confidence $\geq 95 \%$, FDR $=0.05,0.01$, or 0.001 , which matched the same protein entry in the database searched.

\subsection{Binding of rScl proteins to extracellular matrix proteins and synthetic peptide}

3.4.1. ELISA binding assays - Binding of $r S c l$ proteins to extracellular matrix proteins, cellular fibronectin (cFn) and laminin (Lm), was tested by ELISA (35). rScl proteins were immobilized onto Strep-Tactin® coated microplate wells (IBA $\mathrm{GmbH}$ ) at $0.5 \mu \mathrm{M}$ at room temperature for 1.5 hours, then blocked with Tris-buffered saline (TBS) containing 1\% bovine serum albumin (BSA) overnight at $4^{\circ} \mathrm{C}$. The cellular fibronectin from human foreskin fibroblasts (Sigma) and murine laminin (Invitrogen) were added to the wells at 1 $\mu \mathrm{g}$ per well and incubated at room temperature for 1 hour. Bound ECM proteins were detected with rabbit anti-laminin at 1:100 (Sigma) and anti- human fibronectin at 1:4,000 (Sigma) polyclonal antibodies. Secondary antibody goat anti-rabbit $\lg G(H+L) H R P$ conjugate (Bio-Rad) was next used with 1-step ABTS substrate (2,2'-Azinobis [3ethylbenzothiazoline-6-sulfonic acid]-diammonium salt) (ThermoScientific). Absorption was measured using Spectramax 190 at a wavelength of $415 \mathrm{~nm}$. Statistical analysis is based on three independent experiments each containing three technical replicates, using an unpaired $t$-test.

In antibody inhibition assay, the IST-9 mAb targeting the C-C' loop of EDA domain was utilized (37). cFn was either untreated or pre-treated with increasing concentrations of IST-9 blocking mAb $(0.1 \mu \mathrm{g}-1.0 \mu \mathrm{g})$, and added to Strep-Tactin®-coated microplate wells immobilized with rScl proteins, then incubated for 1 hour. Bound ECM proteins were detected as above.

3.4.2. Design and synthesis of the C-C' cyclic peptide - The C-C' cyclic peptide was designed based on the crystal structure of the EDA domain of cFn (PDB code 1J8K). In particular, the region, which was reported to be involved in Scl1 binding, connecting to two $\beta$-strands $C$ and C' of EDA was elongated to the whole C-C' $\beta$-hairpin by allowing the 
formation the electrostatic interaction between Arg33 and Glu45. The terminal Tyr32 and Pro48 were mutated to Cys to stabilize the $\beta$-hairpin by introducing a disulfide bond (Figure S4). The obtained sequence, CRVTYSSPEDGIHELFC (molecular weight: 1997.1 Da), endowed with a cyclic structure to mimic the structure of this region in EDA, was acetylated and amidated at the $\mathrm{N}$ - and C-terminus, respectively. Synthesis of the designed peptide was performed employing the solid phase method on a $50 \mu \mathrm{mol}$ scale initially following standard Fmoc strategies (50). Due to aspartimide formation during traditional acylation reactions, peptide synthesis was carried out employing microwave technology (51). Cyclization was achieved by treating the peptide at $0.1 \mathrm{mg} / \mathrm{mL}$ (to avoid intermolecular disulphide formation) with buffer carbonate $50 \mathrm{mM}, \mathrm{pH}=9$, overnight. The peptide was purified by RP-HPLC and the identity and purity (>97\%) was assessed by LC-MS (data not shown).

\subsubsection{Surface plasmon resonance (SPR) experiments - Real time binding assays} were performed at $25^{\circ} \mathrm{C}$ on a Biacore 3000 Surface Plasmon Resonance (SPR) instrument (GE Healthcare). For immobilization, rScl1.3FL protein containing the Cterminal Strep-tag II was injected at a concentration of $40 \mu \mathrm{M}$ on streptavidin-coated sensor chip, SA Biacore, until the desired level of immobilization was achieved (averaged value of $100 \mathrm{RU}$ ). Binding assays were carried out by injecting the C-C' cyclic peptide at concentrations ranging between 10-500 $\mu \mathrm{M}$. Experiments were carried out in HBS buffer (10 mM HEPES, $150 \mathrm{mM} \mathrm{NaCl}, 3 \mathrm{mM}$ EDTA, pH 7.4). The association phase (kon) was followed for $270 \mathrm{~s}$, whereas the dissociation phase (Koff) was followed for $300 \mathrm{~s}$. The reference chip sensorgrams were subtracted from sample sensorgrams. Experiments were carried out in duplicates. Kinetic parameters were estimated assuming a 1:1 Langmuir binding model and using version 4.1 Evaluation Software (GE Healthcare).

3.4.4. Fluorescence binding analysis - $r S c l 1.3 F L$ protein, at a concentration of $30 \mu \mathrm{M}$, was incubated with increasing concentrations of C-C' cyclic peptide $(0-300 \mu \mathrm{M})$ at 25.0 ${ }^{\circ} \mathrm{C}$, using an excitation wavelength of $298.0 \mathrm{~nm}$ and a fluorescence emission wavelength ranging from 300 to $400 \mathrm{~nm}$. The acquisition parameters were set as follows: excitation 
and emission slits at $5 \mathrm{~nm} ; 120 \mathrm{~nm} / \mathrm{min}$ scan rate; $1.00 \mathrm{~nm}$ data interval averaging time at $0.500 \mathrm{~s}$, PMT voltage at "high". Fluorescence values were recorded at $333 \mathrm{~nm}$, and subtracted from the fluorescence intensity of the ligand-free protein, generating $\Delta$ fluorescence. The - $\Delta$ fluorescence values were plotted against the peptide concentration. (52). Experiments were carried out in duplicates. A control assay was carried out employing the buffer as titrant to assess that the dilution effect was under $3 \%$, not affecting the results.

4. Complementation of GAS strains with full-length Scl1.3FL - For transcomplementation experiments, plasmid pSL502, encoding full-length cell-associated Scl1.3FL protein, was electroporated into MGAS315 WT and MGAS6183 $\Delta s c / 1$ electrocompetent cells. The pJRS525 vector was electroporated as a control. Transformants were selected on $\mathrm{BHI}$ agar containing $100 \mu \mathrm{g} \mathrm{mL}^{-1}$ spectinomycin, and plasmids were re-sequenced. For MGAS10870 $\Delta$ scl1, which contains a spectinomycin resistance cassette in place of the scl1.3 allele, the spectinomycin resistance marker in pSL502 was replaced with a kanamycin resistance, generating the plasmid pSL518 and colonies were selected on $\mathrm{BHI}$ agar containing $150 \mu \mathrm{g} \mathrm{mL}^{-1}$ kanamycin.

\subsection{Determination of Scl1.3- and Scl2.3-protein expression in wild-type and complemented GAS strains}

4.1.1. Western blot analysis - Expression of the Scl1.3 and Scl2.3 proteins was determined by western immunoblotting of the bacterial cell wall and culture supernatant protein fractions, as described before $(29,30)$. Briefly, bacterial cultures were grown to an $\mathrm{OD}_{600}$ of 0.5 and cells were harvested by centrifugation. Culture supernatant proteins were precipitated with trichloroacetic acid (Sigma) to a final concentration of $10 \%$ (vol:vol). The cell wall protein fraction was obtained after cell digestion with lysozyme and mutanolysin in a high sucrose buffer. A total of $10 \mu \mathrm{g}$ of protein samples were separated by SDS-PAGE and transferred to a nitrocellulose membrane. Detection of Scl1.3, Scl2.3, and $\mathrm{M} 3$ proteins was performed using the same sample preparations with rabbit polyclonal antibodies generated against the truncated rScl1.3WT protein (anti-Scl1 
1:15,000 dilution, reported in (40)) and the rScl2.3V region (anti-rScl2.3V 1:2,500; generated by Proteintech (43)). Horseradish peroxidase-conjugated goat anti-rabbit IgG $(\mathrm{H}+\mathrm{L})$ secondary antibody (Bio-Rad), combined with Pierce ${ }^{\mathrm{TM}} \mathrm{ECL}$ western blotting substrate (Thermo Scientific) was used for detection. Images were acquired using a ChemiDoc Touch Imaging System (Bio-Rad).

4.1.2. Flow cytometry analysis - Surface detection of Scl1.3 and Scl2.3 proteins was determined by flow cytometry. Bacteria grown to an $\mathrm{OD}_{600}$ of 0.5 were harvested by centrifugation, and washed with flow cytometry buffer (sterile phosphate-buffered saline containing $10 \%$ Todd-Hewitt broth supplemented with $0.2 \%$ yeast extract). Cells were incubated with polyclonal antibodies against Scl1.3 and Scl2.3 described above at a dilution of 1:100 for 30 minutes on ice, then washed and incubated with Allophycocyanin (APC)-conjugated donkey anti-rabbit IgG $(\mathrm{H}+\mathrm{L})$ (Jackson ImmunoResearch) for 30 minutes on ice. Cells were washed and fixed in $0.4 \%$ paraformaldehyde, and stored at $4^{\circ} \mathrm{C}$ until analysis. Before analysis, cells were washed twice and resuspended in flow cytometry buffer. Cells were analyzed using a BD LSRFortessa, and 50,000 events were collected per sample. Data was analyzed using the FCS Express Flow 5 software.

\section{Assessment of biofilm formation}

5.1. Crystal violet staining assay - Wild-type, mutant, and complemented strains were grown to $\mathrm{OD}_{600}$ of 0.5 and seeded into 24 -well culture plates coated with ECM at $2 \mu \mathrm{g}$ per well, then incubated at $37^{\circ} \mathrm{C}$ with $5 \% \mathrm{CO}_{2}$ for 24 hours. Wells were washed with PBS followed by the addition of $0.5 \mathrm{~mL} 1 \%$ crystal violet solution (Fisher Scientific) diluted in PBS and incubation at room temperature for 30 minutes. Wells were rinsed twice with PBS and stain was solubilized with $0.5 \mathrm{~mL}$ of $75 \%$ ethanol. Spectrophotometric readings were taken for each sample at $\mathrm{OD}_{600}$. Statistical analysis is shown based on three independent experiments, each containing three technical replicates, using an unpaired $t$-test. 
5.2. Confocal laser scanning microscopy (CLSM) - To visualize GAS by CLSM, bacterial cells were transformed with a GFP-encoding plasmid pSB027 (53), as before (36). 15-mm glass cover slips were placed into 24-well tissue culture plate wells and coated with $2 \mu \mathrm{g}$ of cFn or Lm per well. Bacterial cultures grown to logarithmic-phase were added to the wells and allowed to form biofilms for 24 hours. Wells were rinsed with PBS and bacterial cells were fixed with $3 \%$ paraformaldehyde at room temperature for 30 min. Wells were washed again and coverslips were mounted onto slides using ProLong Gold Antifade Mountant (Thermo Scientific). Confocal images were acquired using a $63 \times / 1.40$ Plan-Apochromat objective and a Zeiss LSM 510 laser scanning confocal microscope.

\section{In vitro and in vivo GAS infection models}

6.1. GAS infection of in vitro cultured human skin equivalents - Wounded fullthickness skin equivalents, EpiDerm-FT (MatTek, Boston, MA) were used. The tissues are discs $8 \mathrm{~mm}$ in diameter, which are provided in transwells. A 3-mm wound is generated by performing a punch biopsy to remove the keratinocyte layer. Immediately upon arrival, tissues were equilibrated in antibiotic-free manufacturer's medium overnight at $37^{\circ} \mathrm{C}$ in atmosphere with $5 \% \mathrm{CO}_{2}$. Wounds were infected with $10 \mu \mathrm{L}$ of GFP-expressing log-phase group A streptococcal inocula and incubated in a humid environment at $37^{\circ} \mathrm{C}$ with $5 \%$ $\mathrm{CO}_{2}$ in daily-fresh media; a total of 4 experiments were performed and variables included the inoculum size of $3 \times 10^{6}-1.8 \times 10^{7} \mathrm{CFU}$ and collection time points between 1-5 days. Tissues designated for histopathological evaluation were fixed in $10 \%$ formalin, whereas tissues for two-photon fluorescence (TPF) microscopy imaging were fixed in $4 \%$ paraformaldehyde for several hours and then transferred to petri dishes containing PBS. For visualization of glycocalyx produced by GAS strains, tissues were permeabilized with $0.1 \%$ Triton X-100 in $1 \times$ PBS and blocked with $0.05 \%$ Triton X-100 in $1 \times$ PBS with $1 \%$ BSA before staining with concanavalin A- tetramethylrhodamine (Molecular Probes). Tissues were then rinsed and stored in PBS at $4^{\circ} \mathrm{C}$ until TPF imaging was performed. For TPF analysis, an Olympus 60x/1.2NA water dipping objective was used. Fixed tissues were imaged by two-photon microscopy with the Ti:sapphire laser (Mira, Coherent) 
intensity at $60 \mathrm{~mW}$ and input wavelength of $850 \mathrm{~nm}$. Laser scanning images were collected at 0.5- $1 \mu \mathrm{m}$ incremental depths using Scanlmage (Janelia Farms, HHMI). Images were saved in a single TIFF file with 16 bit depth. Deconvolution of the images was performed using AutoQuant $x 3$ and 3D models of z-stacks were generated using Imaris software.

6.2. Mouse model of soft tissue infection - Animal experiments were conducted in compliance with the regulations and standards under the Animal Welfare Act, the Public Health Service Policy on Humane Care and Use of Laboratory Animals, and the Guide for the Care and Use of Laboratory Animals. The protocol was approved by the West Virginia University Institutional Animal Care and Use Committee (IACUC).

Subcutaneous infections of mice were carried out as described previously (54). Briefly, 5-week-old male, immunocompetent, hairless mice (strain Crl:SKH1-hrBR) were used (Charles River, Wilmington, MA). Groups of 10-15 mice anesthetized with isoflurane were infected subcutaneously at the right flank with $\sim 10^{9}$ GAS CFU of WT or sc/1-mutant strains, and mice were observed for 14 days. The weight and abscess dimensions (length [L] and width [W]) of each mouse were recorded daily during the first week and every other day thereafter. To analyze differences between mice infected with WT and sc/1mutant GAS, the area of each abscess was calculated with the equation for the area $(A)$ of an spherical ellipsoid: $A=\pi(L / 2) \times(W / 2)$; statistical differences were calculated using the student's t-test. At the conclusion of the experiments, mice were anesthetized and sacrificed by cervical dislocation.

\section{RESULTS}

M3-type GAS contain unique insertion of IS1548 element and nonsense mutation within the scl1.3 locus - Since the resurgence of invasive GAS infections in the 1980s and an advent of molecular epidemiology fostered by large-scale sequencing, significant efforts have been made to define the molecular basis for the invasive phenotype of M3type strains. In parallel to these advances, we identified two unique genomic traits in the sc/1.3 locus, encoding streptococcal collagen-like protein 1: (i) the presence of an insertional sequence element, IS1548, in the promoter region and (ii) the presence of a 
nonsense mutation within the coding sequence of $s c / 1.3$ allele that was absent in other M-types.

Studies employing mga-inactivated mutants have indicated that Scl1 expression was positively regulated by the GAS-global transcriptional regulator, Mga $(30,32)$. Two putative Mga binding sites were identified upstream of the scl1 coding sequence in the M1-type strain SF370 and experiments demonstrated that the Mga binding site distal to sc/1 was responsible for transcription activation (34). Identical Mga binding sites I and II were also identified upstream of scl1.3 in the sequenced M3-type strain MGAS315 with IS1548 element inserted 38 bp upstream of the distal Mga I-binding site (Figure 1A). To determine if the IS1548 insertion was specific to M3 strains, we BLAST-searched this element in 45 completed GAS genomes representing 21 different M-types. We observed the presence of IS1548 in all strains searched with varying locations and occurrences from one to twelve per genome (Figure 1B). However, the IS1548 insertion upstream of the scl1.3 allele was only found in the sequenced genomes of M3-type strains MGAS315, SSI-1, and M3-b. Interestingly, a complete IS1548 element was not present upstream of scl1.3 in the recently reported genome of the M3 strain STAB902, which represents a non-invasive isolate (55); instead, a 34-bp remnant of IS1548, including the inverted repeat and additional $14 \mathrm{bp}$, was found. Based on this bioinformatics data, we examined the presence of the IS1548 element upstream of sc/1 by PCR in a panel of 40 M3-type strains, using primers located in the IS1548 and sc/1.3 sequences (IS1548F and Scl1R, Table S1). All M3-type strains examined were positive for the IS1548-sc/1.3 amplicon, while M1, M41, and M28-type controls were negative (Figure 1C), demonstrating a broad and conserved presence of the IS1548 insertion in this location among M3-type GAS.

The nonsense mutation in the $11^{\text {th }}$ Gly-X-Y repeat in the Scl1.3-CL collagenous region (Figure $1 \mathrm{~A}$, red box), presumably results in a truncated secreted variant, which consists of the Scl1-V variable region and $10 \mathrm{Gly}-\mathrm{X}-\mathrm{Y}$ repeats but lacks the cell wall anchor. This polymorphism was originally identified in five M3-type invasive GAS strains but was absent in 45 other strains analyzed, representing 20 different M-types (29). It was later shown in $98.7 \%$ of 479 sequenced invasive M3-type isolates (40). We performed targeted PCR amplification of scl1.3 from 46 additional M3-type strains and observed identical amplicon sizes in all of them (Figure S1A). Sequencing showed that all tested 
strains harbored an identical sc/1.3 allele, containing 25 Gly-X-Y repeats in the collagenous domain with the null mutation in the $11^{\text {th }}$ repeat (Data Set S1). A complete lack of genetic variation within scl1.3 is surprising and differs from the length variation that is commonly observed among $s c$ alleles from other M-types $(29,32,33)$. These results demonstrate that the IS1548 insertion and null mutation we identified in the sc/1.3 locus are unique to and conserved among M3-type GAS. Based on these results, we hypothesized M3 strains produce a truncated, but potentially biologically active Scl1.3 variant, which is secreted instead of being cell-attached.

scl1.3 expression is diminished in M3-type GAS - Original reports showed scl1 transcripts in northern blots, as well as full-length Scl1 proteins (both cell-associated and cell-free fractions) in western blots for strains of emm types 1, 28, 52, and 41 $(29,30,32,45)$. To analyze the expression of the truncated Scl1.3 protein in M3-type GAS, western blot analysis was performed on cell-wall (CW samples) and culture-supernatant (Sup samples) protein fractions of several M3-type strains grown to exponential phase (Figure 2A). The expected truncated Scl1.3 protein was not detected by anti-Scl1 antibodies, whereas the rScl1.3V positive control, corresponding to the $\mathrm{V}$ region of Scl1.3 variant, produced the expected immunoreactive band of $\sim 8.3 \mathrm{kDa}$. In an additional control experiment, the same panel of M3 strains was tested for Scl1.3 on the cell surface using flow cytometry (Figure 2B). No shift in median fluorescence intensity was observed in M3 strains incubated with anti-Scl1 antibody compared to a secondary-only antibody control, indicating a lack of Scl1 on the cell surface among M3 strains.

Given the unique IS1548-scl1.3 location and the lack of truncated Scl1.3 products in culture supernatants, we investigated scl1.3 expression by qRT-PCR. Total RNA was isolated from exponential phase cultures of $5 \mathrm{M} 3$-type strains, as well as from previously characterized control strains of emm types 1, 28, and $41(29,30,32,45)$. Expression of scl1 from each strain was compared to scl1.3 transcription in M3 strain MGAS315 (Figure 2C). Each non-M3-type strain tested had significantly higher transcription level of respective sc/1 allele compared to MGAS315. The M1 strain had the most increased expression by 21 -fold, while M28 and M41 strains exhibited 6 -fold and 8-fold higher sc/1 expression, respectively. Interestingly, the sc/1.3 transcripts examined in four additional 
M3-type MGAS strains $158,335,1313$, and 10870 were significantly reduced as compared to MGAS315, with a range of 25-45-fold decrease. Overall, we observed that M3-type GAS harboring the IS element upstream of sc/1.3 have drastically decreased scl1.3 transcript levels and lack the Scl1 protein product, as assessed by western blot and flow cytometry.

Since $s c / 1$ is regulated by the transcriptional activator Mga, we investigated whether the decreased scl1.3 transcripts in M3 strains were due to lack of mga gene expression in these strains. In parallel, we assessed transcript levels of the emm gene encoding the $\mathrm{M}$ surface protein, a key virulence factor regulated by Mga. For comparison, we included the M1 strain, which had significantly increased scl1 expression compared to the M3 strains. With the exception of MGAS335, which had significantly downregulated $m g a$ and emm expression, we found no significant differences in either mga or emm gene expression between MGAS315 and the other M3 strains or M1 strain (Figure 2D). Furthermore, M3 protein was highly expressed, as it was found in both cell wall and supernatant fractions, except for the MGAS335 strain, consistent with transcription data (Figure 2E). These results demonstrate that the striking downregulation of scl1.3 in M3type GAS is not due to decreased mga expression or non-functional Mga protein, as emm is normally expressed in these strains.

sc/2.3 is expressed in M3-type GAS - Scl2 shares a similar structure with Scl1 but its biological function is poorly understood. One study demonstrated in a different M-type background that isogenic mutant devoid of Scl2.55 variant had lower adhesion to human skin fibroblasts (Rasmussen and Björck, 2001); however, the Scl2.3 variant present in M3-type GAS has not been investigated for expression and ECM binding. Therefore, we next assessed $s c / 2.3 / \mathrm{Scl} 2.3$ expression in M3 strains. PCR amplification and sequencing showed that the majority of M3 strains contained in-frame scl2.3 allele (Figure S1B, Table S2).

Western blot analysis of cell wall (CW samples) and supernatant (Sup) protein fractions found Scl2.3 protein was expressed by M3 strains MGAS315, 10870, 158, 9517 and 1313, whereas samples obtained from strain MGAS335, which contains an out-offrame scl2.3 allele, generated no immunoreactive band; rScl2.3V control produced the 
expected $10.1-\mathrm{kDa}$ band (Figure $3 \mathrm{~A}$ ). Mass spectrometry confirmed the identity of the presumed immunoreactive Scl2.3-protein band from MGAS315 (Table S3). Consistently, Scl2.3 was detected on the surface of all five M3 strains containing in-frame scl2.3 alleles by flow cytometry, with a positive shift in median fluorescence intensity ranging in 67-131fold change, as compared to the secondary-only antibody control (Figure 3B). We next compared the sc/2-transcription level in M3 strain MGAS315 with sc/2-transcription levels in M1-, M28-, and M41-type strains. In striking contrast to the pattern of scl1.3 transcription, scl2 transcripts were significantly decreased in the M1-type strain by 13fold, as well as in the M28 (6-fold) and M41 (3-fold) strains (Figure 3C). Additionally, there was no significant difference between scl2 expression in MGAS315 and the M3 strains MGAS158, 335, 1313, and 10870 (Table S4). These results confirm that the M3 scl2.3 allele is transcribed at high levels, resulting in considerable expression of the Scl2.3 protein. These results suggested that Scl2 has an important biological function in M3 strains and warranted subsequent experiments assessing Scl2.3 function.

\section{GAS infection disseminates through human tissue and inhibits wound healing - A}

wounded human skin equivalent, devoid of an inflammatory component, was utilized as a "mechanistic model" of GAS tissue colonization. The epidermal wound of each skin equivalent was infected with GFP-expressing M3-type invasive strain MGAS315 or M41type non-invasive strain MGAS6183, and analyzed after 1-5 days by standard histopathology (H\&E and Gram's stain) and using two-photon fluorescence (TPF) microscopy. H\&E of uninfected tissue controls harvested at day 0 showed the absence of a keratinocyte layer where the punch biopsy was performed (Figure 4A). Complete healing of the wound was observed after 5 days, with a newly-generated intact keratinocyte layer covering the punch biopsy site (Figure 4B). In contrast, tissue infected with either GAS strain exhibited delayed wound closure as late as day 5 post-infection (Figure 4E, H). H\&E staining of skin equivalents infected with either M3 or M41 after 24 hours revealed bacterial colonization of the exposed dermal surface, as well as bacterial invasion into puncture-associated defects extending deep into the dermal layer, largely located at the wound edges (Figure 4D, G). Notably, extensive bacterial growth and spread was largely confined to tissue crevices in the dermis, whereas the presence of 
large bacterial colonies directly below the wound bed was rarely seen on microscopic examination. By day 5 of infection, bacterial invasion via these dermal defects extended to the bottom of the dermis for both strains, presenting both vertical and lateral spread of bacteria (Figure 4E, H). Gram stain of infected tissues showed the formation of superficial colonies near dermal surfaces, as well as biofilm formation on the surface of exposed dermis (Figure 4J-L). Additionally, epidermal tissue neighboring the wound bed exhibited $\sim 60 \%$ decreased thickness of the viable keratinocyte layer in tissues infected with MGAS315 and MGAS6183 by day 5, relative to uninfected tissues (Figure 4C, F, I). This suggests bacterial infection of the wound affects epidermal cells distant from the site of infection in this model.

TPF analysis was then performed on whole infected skin equivalents on day 5 post-infection in order to assess bacterial spread directly below the wound bed. This method allowed us to visualize bacterial structures within tissue that were not apparent in H\&E or Gram-stained sections. Tissue microcolonies were observed in samples infected with M41 GAS (Figure 4N), whereas M3 cells had a scattered appearance (Figure 4P). TRITC-concanavalin A (TRITC-conA) was utilized to visualize glycocalyx associated with bacteria. TRITC-conA stain colocalized with bacterial microcolonies formed by the M41 GAS (Figure 4O, Figure S3), indicating microcolonies were encased in a glycocalyx, much like a classic biofilm. In contrast, TRITC-conA stain was associated with scattered M3-GAS chains located at the bottom cell layer in MGAS315-infected skin equivalents (Figure 4Q). These results indicate that M41 GAS, but not the M3 GAS, forms microcolonies in the tissue during a human skin infection that are encased in a glycocalyx, consistent with the observation that M41, but not M3, GAS forms robust biofilm in vitro on ECM coatings. In addition, M3 GAS disseminates in a form of scattered chains or single cells through the tissue.

\section{M3-type GAS strains form poor biofilms on extracellular matrix coatings - It was} previously reported that M3-type strains have no substantial biofilm formation in vitro on an inanimate surface (36). Here, we tested biofilm formation on cellular fibronectin (cFn) and laminin $(\mathrm{Lm})$ coatings by a panel of representative M3 strains isolated from invasive cases of GAS disease, as compared to the non-invasive biofilm-capable M41-type model 
strain MGAS6183. As expected, wells coated with either cFn or Lm supported robust biofilm formation by the M41 strain, whereas significantly less bacterial biomass was measured for all M3-type GAS on both ECM coatings (Figure 5A). There was also no correlation between Scl2 expression and biofilm formation. We hypothesized that M3type GAS, devoid of Scl1 adhesin, have decreased binding to cFn and Lm ECM components, thus, preventing the formation of tissue microcolonies, and that restoration of full-length Scl1.3 (Scl1.3FL) on the GAS cell surface will confer binding to host ECM, as well as biofilm capacity in vivo (Figure $5 \mathrm{~B}$ ).

Full-length recombinant Scl1.3 binds cellular fibronectin and laminin - To test this hypothesis, we: (i) constructed Scl1.3- and Scl2.3-derived recombinant proteins, (ii) characterized their structural organization, and (iii) assessed their ECM-binding capacities.

First, rScl1.3FL and rScl2.3 proteins were assessed for purity and integrity by SDSPAGE (Figure 6A). The expected 20.6-kDa rScl1.3FL migrated at $\sim 34 \mathrm{kDa}$, which is consistent with previous reports of aberrant migration of $\mathrm{rScl}$ proteins $(29,30)$, whereas the $\mathrm{rScl} 2.3$ protein migrated according to the expected molecular mass of $16.2 \mathrm{kDa}$; both proteins were verified by mass spectrometry (Table S3). Rotary shadowed rScl1.3FL and rScl2.3 constructs, exhibited the characteristic lollipop-like structural organization (Figure S2), as observed for previously characterized rScl proteins $(47,49)$. Interestingly, rScl1.3FL formed aggregates that were mediated by the intermolecular interactions between the globular domains; such interactions, however, were not observed between rScl2.3 molecules. The appearance of Scl1-Scl1 aggregates implies an attractive hypothesis that V-to-V-region interactions between the Scl1 molecules, but not between the Scl2 molecules, on the surface of neighboring GAS chains may support biofilm structure, as proposed in our model (Figure 5B).

It has been established that Scl1 proteins selectively bind cellular, but not plasma, fibronectin and laminin (35), and that selective cFn binding is achieved by a unique binding mechanism involving the C-C' loop of the extra domain A in cFn (EDA-cFn) (37). Here, we pre-incubated cFn with increasing concentrations of EDA-blocking IST-9 mAb $(0.1,1.0 \mu \mathrm{g})$, then added to wells containing immobilized $\mathrm{rScl}$ proteins and allowed for 
binding. We observed significant dose-dependent inhibition of cFn binding to rScl1.3FL, with a $36 \%$ inhibition at $0.1 \mu \mathrm{g}$ and a $60 \%$ inhibition at $1.0 \mu \mathrm{g}$ of IST-9 (Figure 6B). In addition, the $\mathrm{rScl} 2.3$ protein did not bind $\mathrm{cFn}$, which is consistent with our previous data employing several different $\mathrm{rScl} 2$ proteins (35). Surface plasmon resonance (SPR) measurements of binding affinity between rScl1.3FL and EDA-derived C-C' cyclic peptide provided a dissociation constant of $K_{D}=63.3 \mu \mathrm{M}$ (Figure $6 \mathrm{C}$ ). To corroborate the rScl1.3FL-EDA binding affinity, an in-solution fluorescence binding assay was performed, in which the variation in tryptophan fluorescence of rScl1.3FL was recorded as a measure of positive binding to the C-C' cyclic peptide. Tryptophan fluorescence emission at 333 $n m$ showed a dose-response quenching upon addition of the C-C' cyclic peptide and $-\Delta$ fluorescence intensity was plotted against peptide concentration (Figure 6D). Data were fitted with a 1:1 model of interaction, providing a $K_{D}=44.54 \pm 9 \mu \mathrm{M}$, in agreement with SPR data (56).

We next assessed binding of rScl1.3FL and $\mathrm{rScl} 2.3$ proteins to laminin $(\mathrm{Lm})$ by ELISA. We determined rScl1.3FL had significant Lm binding, whereas rScl2.3 had not (Figure 6E), consistent with previous findings that Scl1-derived recombinant proteins, but not the Scl2-derived, bind ECM proteins (35). Collectively, these results demonstrate specific binding of Scl1.3FL to the EDA domain of cFn and to Lm and its capacity of being surface adhesin.

\section{Homologous complementation of M3 strains with full-length surface-exposed} Scl1.3 adhesin confers biofilm formation on ECM - To assess the effect of cell-surface Scl1.3FL expression on the capacity to form biofilm, an in-trans complementation of two representative invasive M3 strains was performed, MGAS315 wild-type (WT) strain, naturally lacking Scl1.3 expression, and a previously generated sc/1-inactivated mutant of MGAS10870 (10870 $s c / 1)$, with plasmids pSL502 $\left(\mathrm{Sp}^{\mathrm{R}}\right)$ and pSL518 $\left(\mathrm{Km}^{\mathrm{R}}\right)$, respectively, both encoding the full-length Scl1.3FL protein. As a control, MGAS315 was complemented with a shuttle vector pJRS525. The cell wall-associated expression of Scl1.3FL in complemented M3-type GAS was first tested by western blot analysis of the cell wall protein fractions and on the GAS-cell surface by flow cytometry. An expected $\sim 35-k D a$ immunoreactive band in complemented strains was observed, which was absent 
in the parent strains (Figure 7A). Mass spectrometry analysis of the corresponding bands extracted from the gel confirmed they represented the Scl1.3FL protein, with five unique peptides identified with 17\% sequence coverage for complemented MGAS315 and four unique peptides identified with $11 \%$ sequence coverage for complemented MGAS10870 (Table S3). A 6.8-fold increase in median fluorescence intensity of Scl1.3FLcomplemented MGAS315 cells was measured by flow cytometry, as compared to the vector-complemented and WT control strains (Figure 7B). The Scl1.3FL-complemented $10870 \Delta s c / 1$ exhibited a 2.2 -fold increase in median fluorescence intensity, as compared to the mutant control (Figure 7B). These results indicate the Scl1.3FL is indeed expressed and surface-exposed in the complemented M3-type strains.

Biofilm formation by the complemented and parent strains was then assessed after 24 hours following crystal violet staining and using confocal laser scanning microscopy (CLSM). Scl1.3FL-expressing MGAS315 showed significantly increased biomass on both cFn and Lm coatings compared to the WT parent organism, as well as vectorcomplemented control; 3.6 or 2.5 - and 2.5 or 2.1 -fold $\mathrm{OD}_{600}$ increases on cFn and Lm, respectively, were measured compared to MGAS315 WT or to vector-complemented MGAS315 (Figure 7C). Significantly thicker biofilm formed by Scl1.3FL-complemented MGAS315 was imaged by CLSM. We observed on average a 2.8 -fold $(P=0.0002)$ and 2.6-fold ( $P=0.0002)$ increased biofilm thickness on $\mathrm{cFn}$ and $\mathrm{Lm}$, respectively, compared to MGAS315 WT, and a 2.0-fold $(P=0.0036)$ and 4.8 -fold $\left(P=2.1 \times 10^{-5}\right)$ increased biofilm thickness on cFn and Lm, respectively, compared to vector-complemented MGAS315 (Figure 7D and E, representative images). Similar results were obtained for the complemented $10870 \Delta s c / 1$ mutant, which had 1.7-fold increase in biomass staining on both cFn and Lm compared to the MGAS10870 WT strain, and 2.2- and 2.6-fold increase in biomass staining on cFn and Lm, respectively, compared to the $10870 \Delta \mathrm{sc} / 1$ mutant strain (Figure 7C). CLSM data, however, could not be rendered for MGAS10870 strains due to poor GFP expression for unknown reasons. Altogether, it was demonstrated that null mutation in the scl1 gene, which ablates surface Scl1.3 protein and is unique to M3type GAS, is responsible for the decreased biofilm capacity since restoration of the fulllength surface-exposed Scl1.3 adhesin significantly fosters stable biofilm formation. 
Heterologous complementation of M41 $\Delta s c / 1$ mutant strain with full-length surfaceexposed Scl1.3 restores biofilm formation on ECM - In a previous study we showed that sc/1.41-inactivation in a non-invasive biofilm-rich M41 strain MGAS6183 resulted in significantly decreased biofilm capacity, which was restored to wild-type level by complementation with surface Scl1.41 (37). Notably, this M41 strain expresses at least one additional major Fn-binding protein, protein F2 (45), which binds both plasma and cellular fibronectin by a mechanism different from Scl1 (57). Here, we hypothesized that expression of rScl1.3FL in the heterologous M41 GAS will confer biofilm formation. Western immunoblotting of the cell wall protein fractions detected the $\sim 35-\mathrm{kDa}$ immunoreactive band, corresponding to full-length Scl1.3, associated with complemented cells, while the M41 $\Delta s c / 1$ mutant and WT cells were signal-negative (Figure $8 \mathrm{~A}$ ). Mass spectrometry of the corresponding band extracted from the gel confirmed Scl1.3FL expression, with 3 unique peptides identified, covering 13\% of the amino acid sequence (Table S3). In addition, we showed the expression of the larger Scl1.41 variant in the cell wall of M41 WT, but not in the $\Delta s c / 1$ mutant, by re-probing a portion of the blot with antirScl1.41 antibody, using rScl1.41 protein as a positive control.

Similarly to complementation with homologous Scl1.41 protein, the heterologous complementation of the M41 $\Delta s c / 1$ mutant with Scl1.3FL also restored biofilm to M41 WT levels on cFn and Lm (Figure 8B-D). Crystal violet staining showed increased bacterial biomass (Figure $8 \mathrm{~B}$ ) and confocal microscopy revealed significantly thicker biofilms, on average 3-fold increased, as compared to the parental M41 $\Delta s c / 1$ mutant strain on both cFn (Figure 8C) and Lm (Figure 8D) coatings (cFn, $P=0.0105$; Lm, $P=0.0011$ ).

\section{Expression of Scl1 adhesin attenuates GAS during subcutaneous infection - We} have previously shown that an M3 strain harboring a carrier sc/1.3 allele, producing a shorter cell-attached Scl1.3 variant, had an attenuated phenotype in a murine model of necrotizing fasciitis (40). Here, we tested our hypothesis that Scl1 adhesin in biofilm-rich M28 and M41 background promotes stable colonization and localized infection, using a murine skin infection model. Hairless, immunocompetent SKH1 mice were subcutaneously infected with $\sim 10^{9}$ GAS CFU of the M28 and M41 wild-type (WT) or their 
isogenic sc/1-inactivated mutant $(s c / 1)$ strains $(44,45)$, and mice were assessed for changes in gross pathology of the skin.

Skin lesions caused by both the WT and sc/1 strains were observed as early as 48 hours post-infection, and the lesions began to regress after day 7 with complete resolution of the lesion by the completion of the experiment. The areas of the skin lesions calculated for mice infected with the $s c / 1$ mutant strains were significantly larger than those of WTinfected mice (Figure 9A; 96 hour time-point is shown); images of lesions of representative mice demonstrate differences in lesion severity between WT- and sc/1GAS infected mice for both the M28- and M41-infected groups (Figure 9B). Thus, the in vivo data, using GAS strains expressing surface Scl1 proteins and producing rich biofilms, support our hypothesis that decreased adhesion and biofilm formation, due to the absence of Scl1.3 on the surface of M3 strains, bears an inverse correlation to the invasive potential of the infecting GAS strain.

\section{DISCUSSION}

Since the resurgence of invasive GAS disease in the 1980's, and emergence of invasive M3-type isolates, significant efforts have been made to determine the molecular basis for the invasive phenotype of M3-type strains. Numerous whole-genome sequencing projects have identified specific genomic features of M3 strains that were correlated with their potential to cause invasive infections. In this study, we show that Scl1-negative M3type GAS have reduced adhesion and biofilm formation within host tissue, and therefore are predisposed to invasive spread over superficial infection (Figure 10).

Unique topography and expression patterns of $s c /$ loci - First, the insertion of IS1548 element in the promoter region of sc/1 exclusively found in the M3-type strains is an intriguing observation, given the abundance of IS1548 insertions across GAS genomes representing a variety of M-types. IS elements are known to cause genomic rearrangements and affect the expression of important genes that contribute to bacterial virulence (58). For example, the insertion of IS1548 element (59) into the $\operatorname{scpB-Imb}$ intergenic region in group B Streptococcus has been shown to upregulate Imb-gene transcription and Lmb-adhesin surface expression, resulting in enhanced laminin binding 
(60). The IS1548 insertion in the scl1.3 promoter was conserved in the genomes of sequenced M3 strains MGAS315, SSI-1, and M3-b, as well as in 40 additional M3 strains tested by PCR. However, the recently sequenced STAB902 M3 strain (55), which represents a non-invasive isolate, contained a 34-bp IS1548 remnant, suggesting that M3 circulating strains exist with this polymorphism. We hypothesize that the proximity of the IS1548 insertion to the Mga I binding site in M3 strains affects scl1.3 transcription. Comparative qRT-PCR analysis showed significantly higher levels of scl1 transcription, particularly in M1, as well as in M28 and M41 strains, relative to M3-type strains analyzed.

Variation in the Mga coding sequence and autoregulated mga promoter has been reported to significantly affect Mga-dependent gene expression and virulence properties in GAS $(26,27,61)$. For example, a 12-bp deletion of a single repeat in the VNTR region of the mga promoter is present in M3 carrier strains and absent in the invasive M3 strains, causing a downregulation of Mga expression and Mga-regulated genes in the carrier strains (27). We identified an analogous 12-bp deletion in the VNTR of the mga promoter of the M1-type MGAS5005 and in M3 strains, MGAS335 and MGAS1313, but not in MGAS315, MGAS158 and MGAS10870. It is likely that the variation observed in the VNTR region impacts Mga expression but could not, alone, explain differences in sc/1 transcription patterns observed in this work. Consequently, qRT-PCR analyses showed $\mathrm{mga}$ and $\mathrm{emm}$ transcripts (emm is second downstream target of Mga within the Mga regulon) were expressed at similar levels in MGAS5005 and M3 strains, except for the MGAS335, indicating a mechanism of sc/1 downregulation in M3 strains, which is independent from the level of Mga transcription. The presence of emm transcripts and M3-protein products indicate that Mga is present and functional in the majority of M3 strains tested, and therefore the polymorphism we observed in the mga promoter did not affect Mga expression or activity of Mga-controlled scl1.3 promoter. Altogether, our data hint at the insertion of the IS1548 in the downregulation of $s c / 1.3$ expression, specifically in M3-type GAS, although additional studies are required to firmly establish whether the IS element insertion is responsible.

It is intriguing that $s c / 1$ and $s c / 2$ expression patterns were drastically different between M1 and M3-type GAS, both associated with invasive infections. The sc/1.3 transcript was decreased by 21-fold in MGAS315, and even further in the remaining M3 
strains studied (additional 20-45 fold), as compared to the M1-type strain MGAS5005. The upregulation of sc/1 in M1-type GAS has previously been shown in invasive M1 isolates, including MGAS5005, as compared to pharyngeal M1 isolates (62). Studies comparing wild-type and isogenic scl1.1 mutants in M1-type GAS reported that Scl1.1 contributed to immune evasion by inhibiting neutrophil extracellular trap production and by protecting bacteria from the cathelicidin LL-37 (63). We conclude the differences we observed in sc/1 expression are related to different pathogenicity requirements for Scl1 protein in M1- versus M3-type strains. In contrast, Scl2 expression is highly upregulated in M3-type strains, as compared to invasive M1-type GAS. While the majority of M3-type strains contain in-frame scl2.3 alleles, all $21 \mathrm{M} 1$ strains we analyzed contained out-offrame scl2.1 alleles (unpublished data). Although the Scl2.3 human ligands are not known, it may have an unidentified biological function, which is important for pathogenesis of $M 3$, but not M1, strains. Hence, we are reporting striking differences in the expression and features of the Scl1 and Scl2 proteins that evolved in the invasive M1 and M3 strains.

Scl1-mediated adhesion and biofilm formation in vitro - Scl1 mediates binding to human extracellular matrix components, cellular fibronectin and laminin, as well as biofilm formation (35-37). Previous work identified that Scl1 binds specifically to the C-C' loop of the type III-repeat EDA domain of cellular fibronectin (37). This represents a novel mechanism of fibronectin binding, which is distinct from the mechanism employed by other GAS fibronectin-binding proteins that bind to the N-terminal type I repeats of fibronectin (64). Here, we determined that rScl1.3FL binds to cellular fibronectin via the same C-C' loop-dependent mechanism. It is important for our overall model to acknowledge that the EDA-cFn isoform is specifically produced during embryogenesis and during wound healing in adult tissue (65-67), which indicates Scl1 evolved with a unique function for targeting wounded tissue, a presumed pathogen portal of entry. We also demonstrate significant binding of rScl1.3FL to laminin, a major basement membrane protein at the epidermal-dermal junction; none of those ECM ligands were bound by rScl2.3, consistent with previous knowledge that Scl1, but not Scl2, variants exhibit binding to $\mathrm{CFn}$ and Lm (35). These studies show that full-length Scl1.3, if expressed on the M3-GAS cell surface, would bind ECM, contributing to tissue colonization. 
The diminished Scl1.3 expression and poor biofilm formation, led us to hypothesize that lack of surface-expressed Scl1 in M3-type GAS reduces host ECM binding and stable microcolony formation in tissue, thus, shifting the balance towards invasive spread, augmented by other virulence factors expressed by these strains. Recombinant rScl1.3FL formed aggregates that were mediated by the $\mathrm{V}$-to-V region interactions, which could represent a mechanism of biofilm and microcolony stabilization by Scl1 molecules on adjacent GAS cells. Two representative invasive M3 isolates, MGAS315 and MGAS10870, acquired biofilm formation on cFn and $\mathrm{Lm}$ when homologous complementation was performed with surface-attached Scl1.3FL. MGAS315 has been shown to contain a missense mutation in the covS gene, causing upregulation of CovRSregulated virulence genes and enhanced virulence during subcutaneous infection of mice, compared to an isogenic strain containing the WT covS allele (68). Additionally, MGAS315 contains a mutation in the regulator of protein $B$ allele, rop $B$, which produces a nonfunctional RopB variant, while MGAS10870 contains a wild-type covR/S and ropB alleles (28), allowing us to demonstrate the effect of Scl1.3FL function in the presence of differing regulatory networks. Similarly, heterologous complementation with surface Scl1.3FL in a scl1.41-mutant of the non-invasive biofilm-capable strain M41-type MGAS6183, restored its biofilm capacity to the wild-type level. These results indicate that M3-derived Scl1.3FL variant has the full capacity to support biofilm formation to a similar degree as Scl1 from a divergent M-type. The robust biofilm observed in vitro on ECM coatings validates the concept that biofilm capacity combined with adherence to the surrounding host ECM would reinforce the formation of stable tissue microcolonies in vivo.

\section{In vitro skin equivalent model of wound colonization and microcolony formation -} We observed inhibition of wound re-epithelization by GAS infection of wounded skin equivalents, as well as the thinning of the viable epidermal layer at sites distant from the infected wound. In addition to our study, others have reported changes in skin histopathology and wound healing, resulting from bacterial infections. An in vivo study has reported epidermal defects as a result of GAS infection in a humanized mouse model with human skin graft (69). Previous study of M3-type GAS infection using a skin 
equivalent model showed that hyaluronic acid capsule interactions with CD44 receptor on keratinocytes induced intracellular signaling, resulting in cytoskeletal rearrangement and monolayer disruption (70). Infection of an in vitro skin model containing a burn wound with Pseudomonas aeruginosa caused a loss of the keratinocyte layer and basement membrane, while intact epidermis was observed in burned but uninfected tissue (71). Impairment of wound healing has also been demonstrated by staphylococcal infections. Infection of dermal wounds in rabbit ears with Staphylococcus aureus showed the formation of biofilm, production of a persistent, low grade inflammatory response, and significantly delayed wound healing (72). Similarly, delayed wound healing by both $S$. aureus and $S$. epidermidis biofilms was observed in a mouse model of cutaneous wounds (73). The inhibition of wound healing we describe here is by and large consistent with reports by other laboratories generated using in vivo animal and in vitro human skin infection models.

Microcolonies have been identified within human streptococcal impetigo lesions (39) and in tonsils from patients with recurrent GAS pharyngeal tonsillitis (74), and likely represent a superficial or persistent state of GAS colonization. However, streptococcal infections can result in invasive disease due to biofilm disruption and bacterial dissemination $(75,76)$. We observed large rounded microcolonies formed in tissue during infection with M41 strain, while M3 GAS remained scattered throughout the tissue as single cells and chains. Microcolony formation has been previously observed with $S$. aureus infection in organotypic skin model (77). Moreover, wound infection in rabbit ears with $S$. aureus produced mature biofilms encased in exopolysaccharide, as revealed by concanavalin A staining (72). Similarly, we also demonstrated that microcolonies in M41infected tissue were encapsulated in bacterial-associated glycocalyx. However, a lack of glycocalyx-encapsulated microcolonies was associated with infection by M3-type GAS.

These results support our hypothesis that biofilm-poor M3 strains are abolished in stable microcolony formation in vivo, in part due to a lack of the ECM-binding Scl1 protein and an overall lack of surface adhesins, although they likely express the FbaB protein, identified in M3 GAS to be involved in the adherence and invasion into epithelial and endothelial cells (78-80). 
In vivo mouse model of skin infection - Recent study reported that a small proportion of non-invasive M3-type strains ( 1.3\%) were found to harbor the sc/1.3 "carrier allele", which resulted from an in-frame deletion in the collagenous region, encompassing the null mutation, producing a shorter cell-attached Scl1.3 variant. This MGAS10870 strain containing the scl1.3 carrier allele was attenuated following intramuscular infection (40). In this study, we utilized the M28- and M41-type strains, representing biofilm-rich producers, for subcutaneous inoculation. We observed that sc/1.28- and scl1.41inactivated isogenic mutants produced significantly larger skin lesions as compared to the wild-type parent strains. These results, again, support the hypothesis that lack of Scl1 surface adhesin destabilizes focused nidus of infection, resulting in a shift towards increased tissue spread. However, previous studies performed in a M1 GAS background, utilizing scl1.1-mutants for subcutaneous infection, reported smaller skin lesions in the mutant groups, which likely reflects a differing predominant function of Scl1.1 in M1-type GAS $(29,63)$. Investigations using intranasal and intraperitoneal mouse infection models of Streptococcus pneumoniae have shown that culture-grown bacteria disseminated to the ear and lungs, while biofilm-grown bacteria stably colonized the nasopharynx $(81,82)$. A similar study on Streptococcus pyogenes showed that bacteria grown in biofilms have downregulated virulence genes and tend to colonize the nasal associated lymphoid tissue of mice, while culture-grown bacteria had significantly increased dissemination and were more virulent in a septicemia model (83). Previous studies reported that inactivation of some GAS genes resulted in increased skin pathology produced by the mutants compared to their wild-type organisms, and these genes often encoded surface proteins, including SpyCEP (84), Mrp (85), protein F1 (86), and Spy0128, encoding a major pilus subunit (87). Similarly, the covS mutant of group A streptococcal M1T1 strain with upregulated SpeB-protease activity was hypervirulent and had reduced capacity to bind human epithelial cells and fibronectin, and also to form biofilm due to increased cleavage of surface proteins (88). Altogether, the concept that expression of a surface adhesin, such as Scl1, involved in biofilm formation and host tissue attachment, is inversely related to strain invasiveness has gained support from several studies, including this study.

We show the invasive M1- and M3-type GAS evolved sc/1 and scl2 alleles with opposite expression patterns, with sc/1 downregulated and scl2 upregulated in M3 
compared to M1 GAS. We show M3-type GAS, devoid of surface-expressed Scl1.3, lacked biofilm formation on ECM coatings and microcolony formation during infection of in vitro wounded skin equivalent. Complementation with surface Scl1.3FL restored biofilm capacity of M3-type GAS on ECM coatings. Mouse infection with the isogenic scl1 mutants of biofilm-rich M28- and M41-type GAS produced larger lesions, supporting the role of Scl1 in a localized tissue infection. Lastly we developed a model for Scl1-mediated microcolony formation (Figure 10), whereby Scl1 expressed on the GAS surface strengthens host colonization by attachment to cFn and Lm expressed within wounded tissue, as well as biofilm formation via Scl1-Scl1 interactions, resulting in a local, stabilized microcolony. Conversely, when Scl1 is absent on the GAS cell surface, as is the case for M3-type GAS, bacteria lack stable anchoring in the surrounding host ECM, as well as structural strength within microcolony, promoting cells to disperse more freely.

\section{ACKNOWLEDGMENTS}

We thank: Mariette Barbier for assistance with qRT-PCR analysis; Meenal Elliott for assistance with some experiments; Karen Martin, Amanda Ammer, and Lingqing Zhang for assistance with imaging experiments; and Jayme Horning for assistance with mass spectrometry. We thank James Dale for providing anti-M3 antibodies. We also thank Mike Federle and Jennifer Franko for a critical reading of the manuscript. This work was supported in part by National Institutes of Health Grants Al50666 and AI083683 (SL); BB and DHM were supported by the NSF-EPSCoR Graduate Fellowship Program under the Research Infrastructure Improvement (RII) Track-1 award, Cooperative agreement 1003907 (BB) and Integrative Graduate Education and Research Training for Research and Education in Nanotoxicology under award number 1144676 (DHM). BB and DHM were also awarded with the Dr. Jennifer Gossling Scholarship in Microbiology. Flow Cytometry experiments were performed in the West Virginia University Flow Cytometry \& Single Cell Core Facility, which is supported by the National Institutes of Health equipment grant numbers S10OD016165 and RR020866 and the Institutional Development Awards (IDeA) from the National Institute of General Medical Sciences of the National Institutes of Health under grant numbers P30GM103488 (CoBRE) and P20GM103434 (INBRE). Imaging experiments and image analysis were performed in the 
West Virginia University Microscope Imaging Facility, which has been supported by the Mary Babb Randolph Cancer Center and NIH grants P20 RR016440, P30 GM103488 and P20 GM103434.

\section{REFERENCES}

1. Carapetis, J. R., Steer, A. C., Mulholland, E. K., and Weber, M. (2005) The global burden of group A streptococcal diseases. Lancet Infect. Dis. 5, 685-694

2. Sims Sanyahumbi, A., Colquhoun, S., Wyber, R., and Carapetis, J. R. (2016) Global Disease Burden of Group A Streptococcus. in Streptococcus pyogenes: Basic Biology to Clinical Manifestations (Ferretti, J. J., Stevens, D. L., and Fischetti, V. A. eds.), The University of Oklahoma Health Sciences Center, Oklahoma City, OK. pp

3. Cunningham, M. W. (2000) Pathogenesis of group A streptococcal infections. Clin. Microbiol. Rev. 13, 470-511

4. Stevens, D. L., and Kaplan, E. L. (eds). (2000) Streptococcal infections: clinical aspects, microbiology, and molecular pathogenesis, Oxford University Press, New York, N.Y.

5. Shulman, S. T., Tanz, R. R., Kabat, W., Kabat, K., Cederlund, E., Patel, D., Li, Z., Sakota, V., Dale, J. B., and Beall, B. (2004) Group A streptococcal pharyngitis serotype surveillance in North America, 2000-2002. Clin. Infect. Dis. 39, 325-332

6. Musser, J. M., Hauser, A. R., Kim, M. H., Schlievert, P. M., Nelson, K., and Selander, R. K. (1991) Streptococcus pyogenes causing toxic-shock-like syndrome and other invasive diseases: clonal diversity and pyrogenic exotoxin expression. Proc. Natl. Acad. Sci. U. S. A. 88, 2668-2672

7. Cleary, P. P., Kaplan, E. L., Handley, J. P., Wlazlo, A., Kim, M. H., Hauser, A. R., and Schlievert, P. M. (1992) Clonal basis for resurgence of serious Streptococcus pyogenes disease in the 1980s. Lancet 339, 518-521

8. Cockerill, F. R., 3rd, MacDonald, K. L., Thompson, R. L., Roberson, F., Kohner, P. C., BesserWiek, J., Manahan, J. M., Musser, J. M., Schlievert, P. M., Talbot, J., Frankfort, B., Steckelberg, J. M., Wilson, W. R., and Osterholm, M. T. (1997) An outbreak of invasive group A streptococcal disease associated with high carriage rates of the invasive clone among school-aged children. JAMA 277, 38-43

9. Stevens, D. L., Tanner, M. H., Winship, J., Swarts, R., Ries, K. M., Schlievert, P. M., and Kaplan, E. (1989) Severe group A streptococcal infections associated with a toxic shock-like syndrome and scarlet fever toxin A. N. Engl. J. Med. 321, 1-7

10. DiPersio, J. R., File, T. M., Jr., Stevens, D. L., Gardner, W. G., Petropoulos, G., and Dinsa, K. (1996) Spread of serious disease-producing M3 clones of group A Streptococcus among family members and health care workers. Clin. Infect. Dis. 22, 490-495 
11. Johnson, D. R., Stevens, D. L., and Kaplan, E. L. (1992) Epidemiologic analysis of group A streptococcal serotypes associated with severe systemic infections, rheumatic fever, or uncomplicated pharyngitis. J. Infect. Dis. 166, 374-382

12. Davies, H. D., McGeer, A., Schwartz, B., Green, K., Cann, D., Simor, A. E., Low, D. E., and Group, O. G. A. S. S. (1996) Invasive group A streptococcal infections in Ontario, Canada. N. Engl. J. Med. 335, 547-554

13. Kaul, R., McGeer, A., Low, D. E., Green, K., and Schwartz, B. (1997) Population-based surveillance for group A streptococcal necrotizing fasciitis: Clinical features, prognostic indicators, and microbiologic analysis of seventy-seven cases. Ontario Group A Streptococcal Study. Am. J. Med. 103, 18-24

14. Sharkawy, A., Low, D. E., Saginur, R., Gregson, D., Schwartz, B., Jessamine, P., Green, K., and McGeer, A. (2002) Severe group a streptococcal soft-tissue infections in Ontario: 1992-1996. Clin. Infect. Dis. 34, 454-460

15. Hollm-Delgado, M. G., Allard, R., and Pilon, P. A. (2005) Invasive group A streptococcal infections, clinical manifestations and their predictors, Montreal, 1995-2001. Emerg. Infect. Dis. $11,77-82$

16. Gaworzewska, E., and Colman, G. (1988) Changes in the pattern of infection caused by Streptococcus pyogenes. Epidemiol. Infect. 100, 257-269

17. Colman, G., Tanna, A., Efstratiou, A., and Gaworzewska, E. T. (1993) The serotypes of Streptococcus pyogenes present in Britain during 1980-1990 and their association with disease. J. Med. Microbiol. 39, 165-178

18. Meisal, R., Hoiby, E. A., Caugant, D. A., and Musser, J. M. (2010) Molecular characteristics of pharyngeal and invasive emm3 Streptococcus pyogenes strains from Norway, 1988-2003. Eur. J. Clin. Microbiol. Infect. Dis. 29, 31-43

19. Lamagni, T. L., Neal, S., Keshishian, C., Alhaddad, N., George, R., Duckworth, G., VuopioVarkila, J., and Efstratiou, A. (2008) Severe Streptococcus pyogenes infections, United Kingdom, 2003-2004. Emerg. Infect. Dis. 14, 202-209

20. Beres, S. B., Sylva, G. L., Barbian, K. D., Lei, B., Hoff, J. S., Mammarella, N. D., Liu, M. Y., Smoot, J. C., Porcella, S. F., Parkins, L. D., Campbell, D. S., Smith, T. M., McCormick, J. K., Leung, D. Y., Schlievert, P. M., and Musser, J. M. (2002) Genome sequence of a serotype M3 strain of group A Streptococcus: phage-encoded toxins, the high-virulence phenotype, and clone emergence. Proc. Natl. Acad. Sci. U. S. A. 99, 10078-10083

21. Nakagawa, I., Kurokawa, K., Yamashita, A., Nakata, M., Tomiyasu, Y., Okahashi, N., Kawabata, S., Yamazaki, K., Shiba, T., Yasunaga, T., Hayashi, H., Hattori, M., and Hamada, S. (2003) Genome sequence of an M3 strain of Streptococcus pyogenes reveals a large-scale genomic rearrangement in invasive strains and new insights into phage evolution. Genome Res. 13, 10421055 
22. Beres, S. B., Sylva, G. L., Sturdevant, D. E., Granville, C. N., Liu, M., Ricklefs, S. M., Whitney, A. R., Parkins, L. D., Hoe, N. P., Adams, G. J., Low, D. E., DeLeo, F. R., McGeer, A., and Musser, J. M. (2004) Genome-wide molecular dissection of serotype M3 group A Streptococcus strains causing two epidemics of invasive infections. Proc. Natl. Acad. Sci. U. S. A. 101, 11833-11838

23. Beres, S. B., Carroll, R. K., Shea, P. R., Sitkiewicz, I., Martinez-Gutierrez, J. C., Low, D. E., McGeer, A., Willey, B. M., Green, K., Tyrrell, G. J., Goldman, T. D., Feldgarden, M., Birren, B. W., Fofanov, Y., Boos, J., Wheaton, W. D., Honisch, C., and Musser, J. M. (2010) Molecular complexity of successive bacterial epidemics deconvoluted by comparative pathogenomics. Proc. Natl. Acad. Sci. U. S. A. 107, 4371-4376

24. Shea, P. R., Beres, S. B., Flores, A. R., Ewbank, A. L., Gonzalez-Lugo, J. H., Martagon-Rosado, A. J., Martinez-Gutierrez, J. C., Rehman, H. A., Serrano-Gonzalez, M., Fittipaldi, N., Ayers, S. D., Webb, P., Willey, B. M., Low, D. E., and Musser, J. M. (2011) Distinct signatures of diversifying selection revealed by genome analysis of respiratory tract and invasive bacterial populations. Proc. Natl. Acad. Sci. U. S. A. 108, 5039-5044

25. Olsen, R. J., Laucirica, D. R., Watkins, M. E., Feske, M. L., Garcia-Bustillos, J. R., Vu, C., Cantu, C., Shelburne, S. A., 3rd, Fittipaldi, N., Kumaraswami, M., Shea, P. R., Flores, A. R., Beres, S. B., Lovgren, M., Tyrrell, G. J., Efstratiou, A., Low, D. E., Van Beneden, C. A., and Musser, J. M. (2012) Polymorphisms in regulator of protease $B(R o p B)$ alter disease phenotype and strain virulence of serotype M3 group A Streptococcus. J. Infect. Dis. 205, 1719-1729

26. Cao, T. N., Liu, Z., Cao, T. H., Pflughoeft, K. J., Trevino, J., Danger, J. L., Beres, S. B., Musser, J. M., and Sumby, P. (2014) Natural disruption of two regulatory networks in serotype M3 group A Streptococcus isolates contributes to the virulence factor profile of this hypervirulent serotype. Infect. Immun. 82, 1744-1754

27. Flores, A. R., Olsen, R. J., Wunsche, A., Kumaraswami, M., Shelburne, S. A., 3rd, Carroll, R. K., and Musser, J. M. (2013) Natural variation in the promoter of the gene encoding the Mga regulator alters host-pathogen interactions in group a Streptococcus carrier strains. Infect. Immun. 81, 4128-4138

28. Carroll, R. K., Shelburne, S. A., 3rd, Olsen, R. J., Suber, B., Sahasrabhojane, P., Kumaraswami, M., Beres, S. B., Shea, P. R., Flores, A. R., and Musser, J. M. (2011) Naturally occurring single amino acid replacements in a regulatory protein alter streptococcal gene expression and virulence in mice. J. Clin. Invest. 121, 1956-1968

29. Lukomski, S., Nakashima, K., Abdi, I., Cipriano, V. J., Ireland, R. M., Reid, S. D., Adams, G. G., and Musser, J. M. (2000) Identification and characterization of the $s c /$ gene encoding a group $A$ Streptococcus extracellular protein virulence factor with similarity to human collagen. Infect. Immun. 68, 6542-6553

30. Lukomski, S., Nakashima, K., Abdi, I., Cipriano, V. J., Shelvin, B. J., Graviss, E. A., and Musser, J. M. (2001) Identification and characterization of a second extracellular collagen-like protein 
made by group A Streptococcus: control of production at the level of translation. Infect. Immun. 69, 1729-1738

31. Rasmussen, M., and Björck, L. (2001) Unique regulation of SclB - a novel collagen-like surface protein of Streptococcus pyogenes. Mol. Microbiol. 40, 1427-1438

32. Rasmussen, M., Edén, A., and Björck, L. (2000) SclA, a novel collagen-like surface protein of Streptococcus pyogenes. Infect. Immun. 68, 6370-6377

33. Whatmore, A. M. (2001) Streptococcus pyogenes sc/B encodes a putative hypervariable surface protein with a collagen-like repetitive structure. Microbiology 147, 419-429

34. Almengor, A. C., and Mclver, K. S. (2004) Transcriptional activation of $s c / A$ by Mga requires a distal binding site in Streptococcus pyogenes. J. Bacteriol. 186, 7847-7857

35. Caswell, C. C., Oliver-Kozup, H., Han, R., Lukomska, E., and Lukomski, S. (2010) Scl1, the multifunctional adhesin of group A Streptococcus, selectively binds cellular fibronectin and laminin, and mediates pathogen internalization by human cells. FEMS Microbiol. Lett. 303, 61-68

36. Oliver-Kozup, H. A., Elliott, M., Bachert, B. A., Martin, K. H., Reid, S. D., Schwegler-Berry, D. E., Green, B. J., and Lukomski, S. (2011) The streptococcal collagen-like protein-1 (Scl1) is a significant determinant for biofilm formation by group A Streptococcus. BMC Microbiol. 11, 262

37. Oliver-Kozup, H., Martin, K. H., Schwegler-Berry, D., Green, B. J., Betts, C., Shinde, A. V., Van De Water, L., and Lukomski, S. (2013) The group A streptococcal collagen-like protein-1, Scl1, mediates biofilm formation by targeting the extra domain A-containing variant of cellular fibronectin expressed in wounded tissue. Mol. Microbiol. 87, 672-689

38. Lembke, C., Podbielski, A., Hidalgo-Grass, C., Jonas, L., Hanski, E., and Kreikemeyer, B. (2006) Characterization of biofilm formation by clinically relevant serotypes of group A streptococci. Appl. Environ. Microbiol. 72, 2864-2875

39. Akiyama, H., Morizane, S., Yamasaki, O., Oono, T., and Iwatsuki, K. (2003) Assessment of Streptococcus pyogenes microcolony formation in infected skin by confocal laser scanning microscopy. J. Dermatol. Sci. 32, 193-199

40. Flores, A. R., Jewell, B. E., Versalovic, E. M., Olsen, R. J., Bachert, B. A., Lukomski, S., and Musser, J. M. (2015) Natural variant of collagen-like protein A in serotype M3 Group A Streptococcus increases adherence and decreases invasive potential. Infect. Immun. 83, 11221129

41. Påhlman, L. I., Marx, P. F., Mörgelin, M., Lukomski, S., Meijers, J. C., and Herwald, H. (2007) Thrombin-activatable fibrinolysis inhibitor binds to Streptococcus pyogenes by interacting with collagen-like proteins A and B. J Biol Chem 282, 24873-24881

42. Squeglia, F., Bachert, B., Romano, M., Lukomski, S., and Berisio, R. (2013) Crystallization and preliminary X-ray crystallographic analysis of the variable domain of Scl2.3, a streptococcal collagen-like protein from invasive M3-type Streptococcus pyogenes. Acta Crystallogr. Sect. F Struct. Biol. Cryst. Commun. 69, 1023-1025 
43. Squeglia, F., Bachert, B., De Simone, A., Lukomski, S., and Berisio, R. (2014) The crystal structure of the streptococcal collagen-like protein 2 globular domain from invasive M3-type group A Streptococcus shows significant similarity to immunomodulatory HIV protein gp41. J Biol Chem 289, 5122-5133

44. Han, R., Caswell, C. C., Lukomska, E., Keene, D. R., Pawlowski, M., Bujnicki, J. M., Kim, J. K., and Lukomski, S. (2006) Binding of the low-density lipoprotein by streptococcal collagen-like protein Scl1 of Streptococcus pyogenes. Mol. Microbiol. 61, 351-367

45. Caswell, C. C., Lukomska, E., Seo, N. S., Höök, M., and Lukomski, S. (2007) Scl1-dependent internalization of group A Streptococcus via direct interactions with the $\alpha 2 \beta 1$ integrin enhances pathogen survival and re-emergence. Mol. Microbiol. 64, 1319-1331

46. Virtaneva, K., Porcella, S. F., Graham, M. R., Ireland, R. M., Johnson, C. A., Ricklefs, S. M., Babar, I., Parkins, L. D., Romero, R. A., Corn, G. J., Gardner, D. J., Bailey, J. R., Parnell, M. J., and Musser, J. M. (2005) Longitudinal analysis of the group A Streptococcus transcriptome in experimental pharyngitis in cynomolgus macaques. Proc. Natl. Acad. Sci. U. S. A. 102, 90149019

47. Han, R., Zwiefka, A., Caswell, C. C., Xu, Y., Keene, D. R., Lukomska, E., Zhao, Z., Höök, M., and Lukomski, S. (2006) Assessment of prokaryotic collagen-like sequences derived from streptococcal Scl1 and Scl2 proteins as a source of recombinant GXY polymers. Appl. Microbiol. Biotechnol. 72, 109-115

48. Mclver, K. S., and Scott, J. R. (1997) Role of $m g a$ in growth phase regulation of virulence genes of the group A Streptococcus. J. Bacteriol. 179, 5178-5187

49. Xu, Y., Keene, D. R., Bujnicki, J. M., Höök, M., and Lukomski, S. (2002) Streptococcal Scl1 and Scl2 proteins form collagen-like triple helices. J Biol Chem 277, 27312-27318

50. Fields, G. B., and Noble, R. L. (1990) Solid phase peptide synthesis utilizing 9fluorenylmethoxycarbonyl amino acids. Int. J. Pept. Protein Res. 35, 161-214

51. Vanier, G. S. (2013) Microwave-assisted solid-phase peptide synthesis based on the Fmoc protecting group strategy (CEM). Methods Mol. Biol. 1047, 235-249

52. Williamson, M. P. (2013) Using chemical shift perturbation to characterise ligand binding. Progress in nuclear magnetic resonance spectroscopy 73, 1-16

53. Cramer, T., Yamanishi, Y., Clausen, B. E., Förster, I., Pawlinski, R., Mackman, N., Haase, V. H., Jaenisch, R., Corr, M., Nizet, V., Firestein, G. S., Gerber, H. P., Ferrara, N., and Johnson, R. S. (2003) HIF-1 $\alpha$ is essential for myeloid cell-mediated inflammation. Cell 112, 645-657

54. Lukomski, S., Montgomery, C. A., Rurangirwa, J., Geske, R. S., Barrish, J. P., Adams, G. J., and Musser, J. M. (1999) Extracellular cysteine protease produced by Streptococcus pyogenes participates in the pathogenesis of invasive skin infection and dissemination in mice. Infect. Immun. 67, 1779-1788 
55. Soriano, N., Vincent, P., Moullec, S., Meygret, A., Lagente, V., Kayal, S., and Faili, A. (2014) Closed genome sequence of noninvasive Streptococcus pyogenes M/emm3 strain STAB902. Genome announcements 2

56. Russo, A., Scognamiglio, P. L., Hong Enriquez, R. P., Santambrogio, C., Grandori, R., Marasco, D., Giordano, A., Scoles, G., and Fortuna, S. (2015) In Silico Generation of Peptides by Replica Exchange Monte Carlo: Docking-Based Optimization of Maltose-Binding-Protein Ligands. PLoS One 10, e0133571

57. Sela, S., Aviv, A., Tovi, A., Burstein, I., Caparon, M. G., and Hanski, E. (1993) Protein F: an adhesin of Streptococcus pyogenes binds fibronectin via two distinct domains. Mol. Microbiol. 10, 1049-1055

58. Mahillon, J., Leonard, C., and Chandler, M. (1999) IS elements as constituents of bacterial genomes. Res. Microbiol. 150, 675-687

59. Granlund, M., Oberg, L., Sellin, M., and Norgren, M. (1998) Identification of a novel insertion element, IS1548, in group B streptococci, predominantly in strains causing endocarditis. J. Infect. Dis. 177, 967-976

60. Al Safadi, R., Amor, S., Hery-Arnaud, G., Spellerberg, B., Lanotte, P., Mereghetti, L., Gannier, F., Quentin, R., and Rosenau, A. (2010) Enhanced expression of Imb gene encoding laminin-binding protein in Streptococcus agalactiae strains harboring IS1548 in scpB-Imb intergenic region. PLoS One 5, e10794

61. Sanson, M., O'Neill, B. E., Kachroo, P., Anderson, J. R., Flores, A. R., Valson, C., Cantu, C. C., Makthal, N., Karmonik, C., Fittipaldi, N., Kumaraswami, M., Musser, J. M., and Olsen, R. J. (2015) A naturally occurring single amino acid replacement in multiple gene regulator of group $A$ Streptococcus significantly increases virulence. Am. J. Pathol. 185, 462-471

62. Sumby, P., Whitney, A. R., Graviss, E. A., DeLeo, F. R., and Musser, J. M. (2006) Genome-wide analysis of group A streptococci reveals a mutation that modulates global phenotype and disease specificity. PLoS Pathog. 2, e5

63. Dohrmann, S., Anik, S., Olson, J., Anderson, E. L., Etesami, N., No, H., Snipper, J., Nizet, V., and Okumura, C. Y. (2014) Role for streptococcal collagen-like protein 1 in M1T1 group A Streptococcus resistance to neutrophil extracellular traps. Infect. Immun. 82, 4011-4020

64. Yamaguchi, M., Terao, Y., and Kawabata, S. (2013) Pleiotropic virulence factor - Streptococcus pyogenes fibronectin-binding proteins. Cell. Microbiol. 15, 503-511

65. Singh, P., Reimer, C. L., Peters, J. H., Stepp, M. A., Hynes, R. O., and Van De Water, L. (2004) The spatial and temporal expression patterns of integrin $\alpha 9 \beta 1$ and one of its ligands, the EIIIA segment of fibronectin, in cutaneous wound healing. J. Invest. Dermatol. 123, 1176-1181

66. Ffrench-Constant, C., Van De Water, L., Dvorak, H. F., and Hynes, R. O. (1989) Reappearance of an embryonic pattern of fibronectin splicing during wound healing in the adult rat. J. Cell Biol. 109, 903-914 
67. Jarnagin, W. R., Rockey, D. C., Koteliansky, V. E., Wang, S. S., and Bissell, D. M. (1994) Expression of variant fibronectins in wound healing: cellular source and biological activity of the EIIIA segment in rat hepatic fibrogenesis. J. Cell Biol. 127, 2037-2048

68. Stetzner, Z. W., Li, D., Feng, W., Liu, M., Liu, G., Wiley, J., and Lei, B. (2015) Serotype M3 and M28 group A streptococci have distinct capacities to evade neutrophil and TNF-alpha responses and to invade soft tissues. PLoS One 10, e0129417

69. Scaramuzzino, D. A., McNiff, J. M., and Bessen, D. E. (2000) Humanized in vivo model for streptococcal impetigo. Infect. Immun. 68, 2880-2887

70. Cywes, C., and Wessels, M. R. (2001) Group A Streptococcus tissue invasion by CD44-mediated cell signalling. Nature 414, 648-652

71. Shepherd, J., Douglas, I., Rimmer, S., Swanson, L., and MacNeil, S. (2009) Development of three-dimensional tissue-engineered models of bacterial infected human skin wounds. Tissue Eng Part C Methods 15, 475-484

72. Gurjala, A. N., Geringer, M. R., Seth, A. K., Hong, S. J., Smeltzer, M. S., Galiano, R. D., Leung, K. P., and Mustoe, T. A. (2011) Development of a novel, highly quantitative in vivo model for the study of biofilm-impaired cutaneous wound healing. Wound Repair Regen. 19, 400-410

73. Schierle, C. F., De la Garza, M., Mustoe, T. A., and Galiano, R. D. (2009) Staphylococcal biofilms impair wound healing by delaying reepithelialization in a murine cutaneous wound model. Wound Repair Regen. 17, 354-359

74. Roberts, A. L., Connolly, K. L., Kirse, D. J., Evans, A. K., Poehling, K. A., Peters, T. R., and Reid, S. D. (2012) Detection of group A Streptococcus in tonsils from pediatric patients reveals high rate of asymptomatic streptococcal carriage. BMC Pediatr. 12, 3

75. Connolly, K. L., Roberts, A. L., Holder, R. C., and Reid, S. D. (2011) Dispersal of Group A streptococcal biofilms by the cysteine protease SpeB leads to increased disease severity in a murine model. PLoS One 6, e18984

76. Connolly, K. L., Braden, A. K., Holder, R. C., and Reid, S. D. (2011) Srv mediated dispersal of streptococcal biofilms through SpeB is observed in CovRS+ strains. PLoS One 6, e28640

77. Popov, L., Kovalski, J., Grandi, G., Bagnoli, F., and Amieva, M. R. (2014) Three-Dimensional Human Skin Models to Understand Staphylococcus aureus Skin Colonization and Infection.

Front. Immunol. 5, 41

78. Terao, Y., Kawabata, S., Nakata, M., Nakagawa, I., and Hamada, S. (2002) Molecular characterization of a novel fibronectin-binding protein of Streptococcus pyogenes strains isolated from toxic shock-like syndrome patients. J Biol Chem 277, 47428-47435

79. Terao, Y., Kawabata, S., Kunitomo, E., Murakami, J., Nakagawa, I., and Hamada, S. (2001) Fba, a novel fibronectin-binding protein from Streptococcus pyogenes, promotes bacterial entry into epithelial cells, and the fba gene is positively transcribed under the Mga regulator. Mol. Microbiol. 42, 75-86 
80. Amelung, S., Nerlich, A., Rohde, M., Spellerberg, B., Cole, J. N., Nizet, V., Chhatwal, G. S., and Talay, S. R. (2011) The FbaB-type fibronectin-binding protein of Streptococcus pyogenes promotes specific invasion into endothelial cells. Cell. Microbiol. 13, 1200-1211

81. Marks, L. R., Davidson, B. A., Knight, P. R., and Hakansson, A. P. (2013) Interkingdom signaling induces Streptococcus pneumoniae biofilm dispersion and transition from asymptomatic colonization to disease. MBio 4, e00438-00413

82. Blanchette-Cain, K., Hinojosa, C. A., Akula Suresh Babu, R., Lizcano, A., Gonzalez-Juarbe, N., Munoz-Almagro, C., Sanchez, C. J., Bergman, M. A., and Orihuela, C. J. (2013) Streptococcus pneumoniae biofilm formation is strain dependent, multifactorial, and associated with reduced invasiveness and immunoreactivity during colonization. MBio 4, e00745-00713

83. Marks, L. R., Mashburn-Warren, L., Federle, M. J., and Hakansson, A. P. (2014) Streptococcus pyogenes biofilm growth in vitro and in vivo and its role in colonization, virulence, and genetic exchange. J. Infect. Dis. 210, 25-34

84. Sumby, P., Zhang, S., Whitney, A. R., Falugi, F., Grandi, G., Graviss, E. A., Deleo, F. R., and Musser, J. M. (2008) A chemokine-degrading extracellular protease made by group A Streptococcus alters pathogenesis by enhancing evasion of the innate immune response. Infect. Immun. 76, 978-985

85. Boyle, M. D., Raeder, R., Flosdorff, A., and Podbielski, A. (1998) Role of emm and mrp genes in the virulence of group A streptococcal isolate $64 / 14$ in a mouse model of skin infection. J. Infect. Dis. 177, 991-997

86. Nyberg, P., Sakai, T., Cho, K. H., Caparon, M. G., Fassler, R., and Bjorck, L. (2004) Interactions with fibronectin attenuate the virulence of Streptococcus pyogenes. EMBO J. 23, 2166-2174

87. Crotty Alexander, L. E., Maisey, H. C., Timmer, A. M., Rooijakkers, S. H., Gallo, R. L., von Kockritz-Blickwede, M., and Nizet, V. (2010) M1T1 group A streptococcal pili promote epithelial colonization but diminish systemic virulence through neutrophil extracellular entrapment. J. Mol. Med. (Berl.) 88, 371-381

88. Hollands, A., Pence, M. A., Timmer, A. M., Osvath, S. R., Turnbull, L., Whitchurch, C. B., Walker, M. J., and Nizet, V. (2010) Genetic switch to hypervirulence reduces colonization phenotypes of the globally disseminated group A Streptococcus M1T1 clone. J. Infect. Dis. 202, 11-19 


\section{FIGURES}

\section{Figure 1}

A

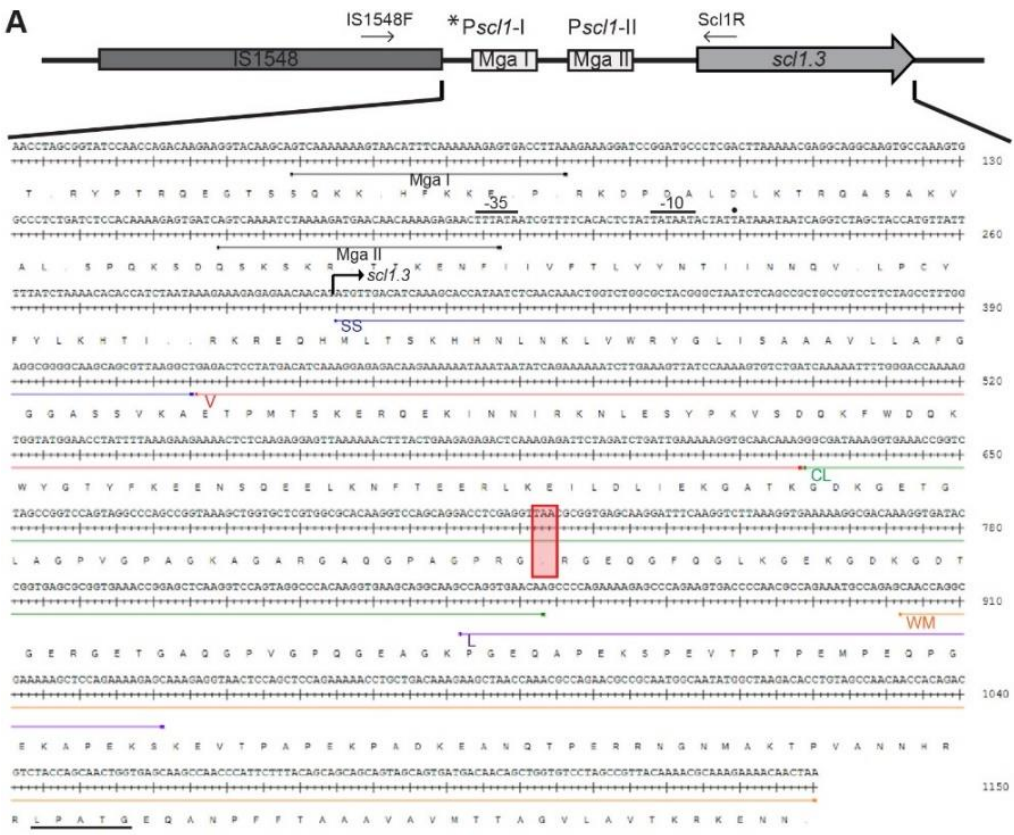

C

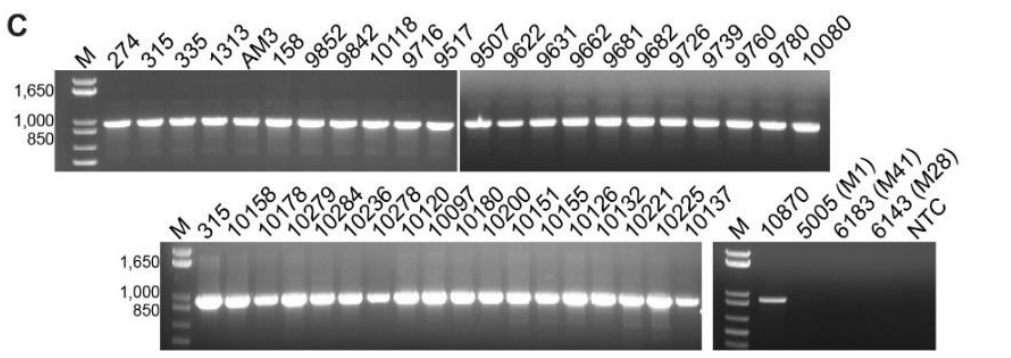

B

\begin{tabular}{|l|l|l|l|}
\hline Strain & M-type & $\begin{array}{l}\text { No. of } \\
\text { insertions }\end{array}$ & $\begin{array}{l}\text { Upstream of } \\
\text { scl1 allele? }\end{array}$ \\
\hline M1 GAS (SF370) & M1 & 6 & - \\
\hline MGAS476 & M1 & 8 & - \\
\hline MGAS5005 & M1 & 9 & - \\
\hline HKU488 & M1 & 7 & - \\
\hline 5448 & M1 & 7 & - \\
\hline MTB314 & M1 & 7 & - \\
\hline A20 & M1 & 8 & - \\
\hline AP1 & M1 & 5 & - \\
\hline NCTC8198 & M1 & 5 & - \\
\hline MTB313 & M1 & 7 & - \\
\hline MGAS10270 & M2 & 2 & - \\
\hline MGAS315 & M3 & 3 & + \\
\hline SSI-1 & M3 & 3 & - \\
\hline M3-b & M3 & 3 & - \\
\hline STAB902 & M3 & 2 & - \\
\hline MGAS10750 & M4 & 9 & - \\
\hline MEW427 & M4 & 5 & - \\
\hline ATCC19615 & M5/49 & 4 & - \\
\hline MGAS10394 & M6 & 2 & - \\
\hline D471 & M6 & 2 & - \\
\hline JRS4 & M6 & 2 & - \\
\hline MGAS2096 & M12 & 7 & - \\
\hline MGAS9429 & M12 & 8 & - \\
\hline HKU360 & M12 & 7 & - \\
\hline HSC5 & M14 & 3 & - \\
\hline M23ND & M23 & 1 & - \\
\hline FDAARG0S_149 & M24 & 9 & - \\
\hline MGAS6180 & M28 & 4 & - \\
\hline MEW123 & M28 & 4 & - \\
\hline M28PF1 & M28 & 4 & - \\
\hline STAB10015 & M28 & 4 & - \\
\hline 1E1 & M44 & 1 & - \\
\hline STAB901 & M44 & 1 & - \\
\hline NZ131 & M49 & 4 & - \\
\hline Alab49 & M53 & 12 & - \\
\hline MGAS15252 & M59 & 5 & - \\
\hline MGAS1882 & M59 & 6 & - \\
\hline NGAS596 & M82 & 7 & - \\
\hline 7F7 & M83 & 2 & - \\
\hline STAB1102 & M83 & 2 & - \\
\hline NGAS327 & M83 & 2 & - \\
\hline NGAS743 & M87 & 12 & - \\
\hline H293 & & & - \\
\hline NGAS638 & M14 & 7 & - \\
\hline NGAS322 & & - \\
\hline
\end{tabular}

Figure 1: M3-type GAS strains harbor unique polymorphisms in the scl1.3 locus. (A) Schematic representation and nucleotide sequence are based on the sc/1.3 locus in the M3-type strain MGAS315 genome. The scl1.3 promoter region contains two putative Mga binding sites, Psc/1-I with Mga I and Psc/1II with Mga II. IS1548 is inserted 38 bp upstream of the preferred * $\mathrm{Psc} / 1-\mathrm{I} / \mathrm{Mga}$ I promoter, which was shown to be necessary for $s c / 1$ transcription. Transcriptional start site (solid dot), -10 and -35 boxes are shown upstream of scl1.3 coding sequence. Scl1 regions are designated as follows: SS, signal sequence; $\mathrm{V}$, variable region; $C L$, collagen-like region; $L$, linker region; WM, wall-membrane region; LPATG, cell-wall anchor. The null mutation in the $11^{\text {th }}$ Gly-X-Y repeat of the $C L$ region is depicted by the red box, presumably resulting in a truncated secreted Scl1.3 protein. Relative location of primers, IS1548F and Scl1R, used to generate amplicons in (C) are shown. (B) IS1548 insertion upstream of $s c / 1.3$ is unique to M3 genomes. BLAST search in the NCBI nucleotide ( $\mathrm{nr} / \mathrm{nt})$ database using IS1548 (1,317 bp) sequence as query identified insertions in 45 GAS genomes representing 21 different M-types. Only genomes of M3-type 
strains harbored IS1548 element upstream of the sc/1 allele (bold text). (C) IS1548 insertion upstream of sc/1.3 is conserved among M3 strains. Genomic DNA was isolated from a collection of 40 M3-type strains and analyzed by PCR for the presence of IS1548 upstream of sc/1 using primers IS1548F and Scl1R (located in conserved sc/1 signal sequence). Additional M1-, M41-, and M28-type control strains, and a no template control (NTC) are included. Expected amplicon size, 963 bp; M, 1 kb Plus DNA Ladder. MGAS designation applies to all strain numbers shown above gel wells, with the exception of strain AM3. 


\section{Figure 2}

A

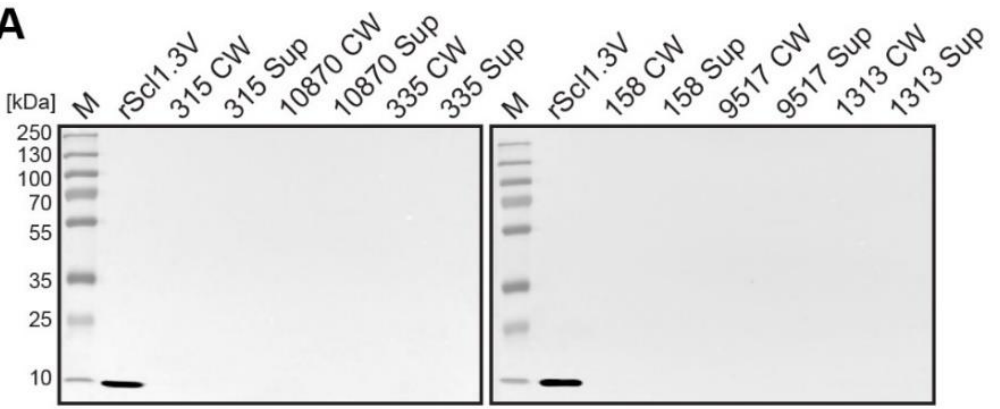

C

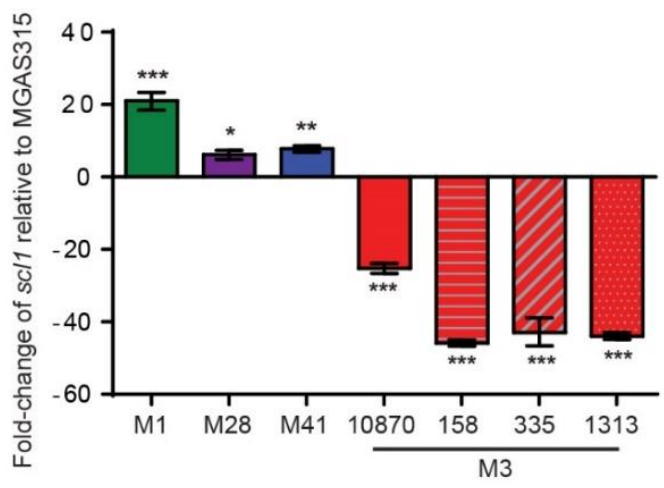

E

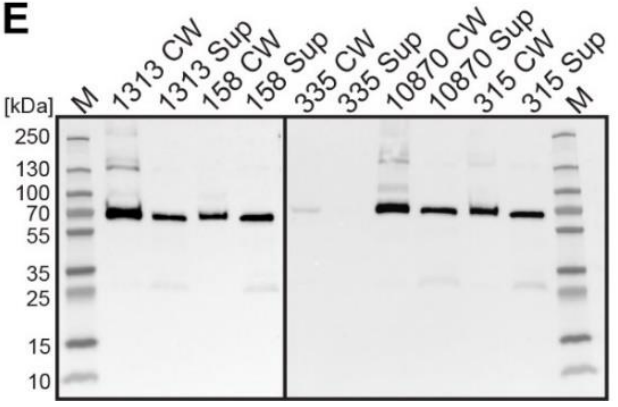

B
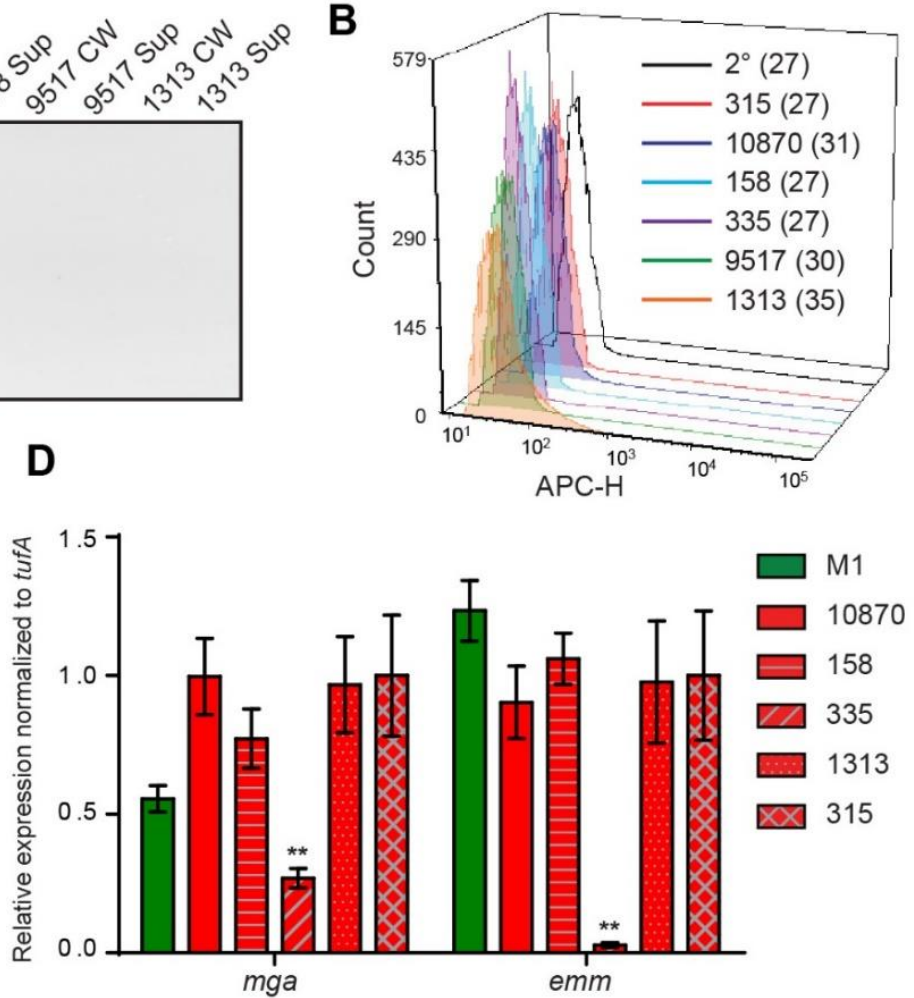

Figure 2: Assessment of Scl1.3 expression. (A) Assessment of Scl1.3 production by M3-type GAS. Cell wall (CW) and culture supernatant (Sup) protein fractions prepared from exponential phase cultures of several M3-type strains were analyzed by western immunoblotting, using anti-Scl1 rabbit polyclonal antibody. Recombinant protein rScl1.3V, corresponding to the variable region of Scl1.3, was used as a positive control. Expected molecular masses: Scl1.3, 11.4 kDa; rScl1.3V, 8.3 kDa. M, PageRuler ${ }^{\mathrm{TM}}$ Plus Prestained Protein Ladder. $(B)$ Detection of Scl1.3 on the surface of M3-type GAS. Flow cytometry analysis of several M3-type strains is shown using anti-Scl1 antibody described in part (A) (color-shaded histograms) or a secondary only control ( $2^{\circ}$ sample, black outlined histogram). Median fluorescence intensities (MFI) are shown in parentheses for each strain. (C) Assessment of sc/1 transcription. Fold-change of sc/1 transcript levels are shown compared to sc/1.3 transcription in M3-type strain MGAS315. qRT-PCR was 
performed on RNA obtained from exponential phase cultures. Results are shown from three independent experiments, each performed in triplicate wells. Standard errors and statistical analysis were computed from averaged $\triangle \mathrm{Ct}$ values for each biological replicate prior to normalization against the endogenous reference gene tufA; ${ }^{*} P \leq 0.05,{ }^{* *} P \leq 0.01,{ }^{* * *} P \leq 0.001$ (student's $t$-test). (D) Assessment of $m g a$ and emm transcription. Relative expression levels of $m g a$ and emm genes were compared between MGAS315 and four additional M3 strains or the M1 strain MGAS5005. Results are shown from three independent experiments, each performed in triplicate wells. Standard errors and statistical analysis were computed from averaged $\triangle \mathrm{Ct}$ values for each biological replicate prior to normalization against the endogenous reference gene tufA; ${ }^{\star \star} P \leq 0.01$. (E) Assessment of M3-protein production by M3-type GAS. The same cell wall (CW) and culture supernatant (Sup) protein samples prepared from exponential phase cultures of M3type strains (used in panel A) were analyzed by western immunoblotting, using anti-M3 protein rabbit polyclonal antibody. Expected molecular mass: $65 \mathrm{kDa}$. M, PageRuler ${ }^{\mathrm{TM}}$ Plus Prestained Protein Ladder. 


\section{Figure 3:}

A
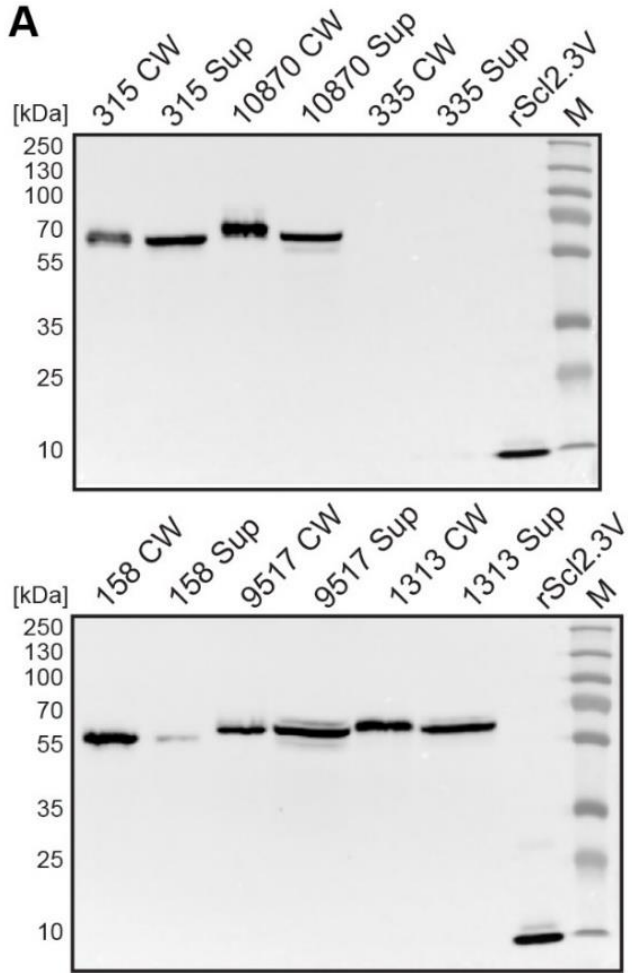

B

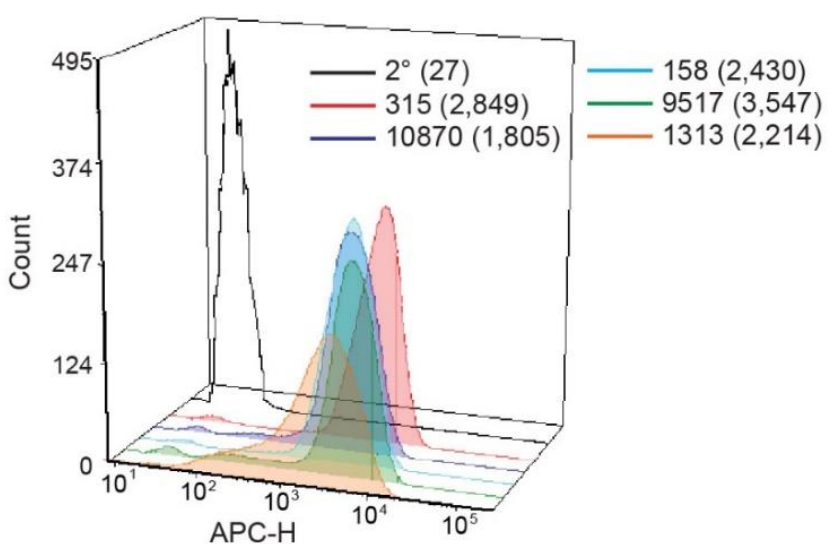

C

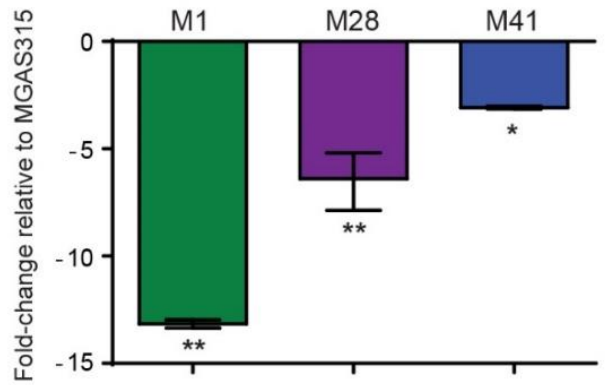

Figure 3: Characterization of the sc/2.3 locus in M3-type GAS. (A) Assessment of Scl2.3 production by M3-type GAS. The same cell wall (CW) and culture supernatant (Sup) protein samples prepared from exponential phase cultures of several M3-type strains (used in Fig. 2A, E) were analyzed by western immunoblotting, using anti-rScl2.3V rabbit polyclonal antibody. Recombinant protein rScl2.3V, corresponding to the variable region of Scl2.3 protein, was used as a positive control. Expected molecular masses based on MGAS315: Scl2.3, $52.5 \mathrm{kDa}$; rScl2.3V, 10.1 kDa. Aberrant migration of detected Scl2.3 variants is characteristic of Scl proteins. M, PageRulerTM Plus Prestained Protein Ladder. $(B)$ Detection of Scl2.3 on the surface of M3-type GAS. Flow cytometry analysis of several M3-type strains is shown using anti-rScl2.3V rabbit polyclonal antibody (color-shaded histograms) or a secondary-only control ( $2^{\circ}$ sample, black outlined histogram). Median fluorescence intensities (MFI) are shown in parentheses for each strain. (C) Assessment of $s c / 2$ transcription. Fold-change of $s c / 2$ transcription levels are shown compared to $s c / 2.3$ transcription in M3-type MGAS315. qRT-PCR was performed on reverse-transcribed RNA obtained from exponential phase cultures. Results are shown from three independent experiments, each performed in triplicate wells. Standard errors and statistical analysis were computed from averaged $\triangle \mathrm{Ct}$ values for each biological replicate prior to normalization against the endogenous reference gene tufA; ${ }^{*} P \leq 0.05,{ }^{* *} P \leq 0.01$ (student's $t$-test). 
Figure 4

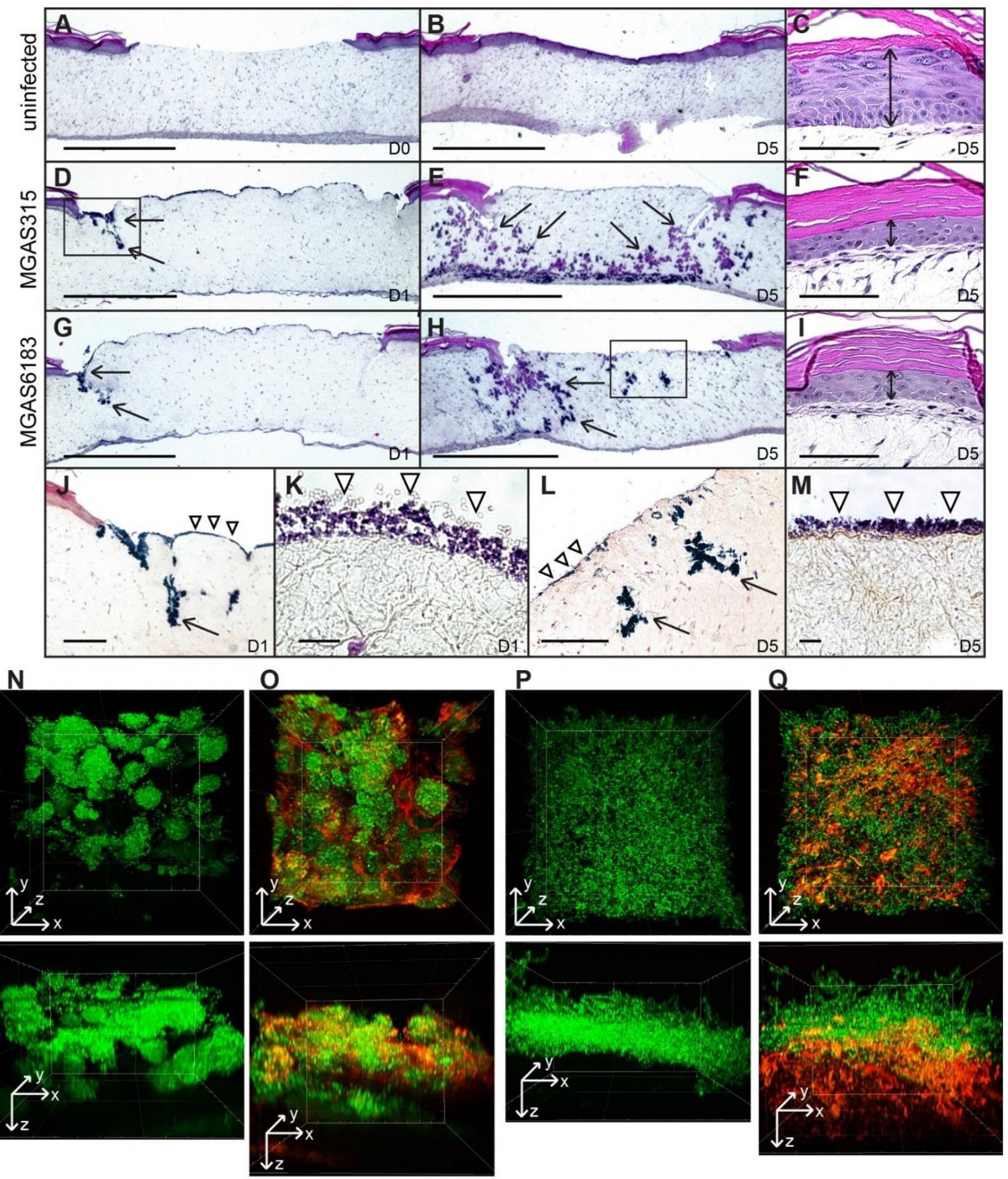

Figure 4: In vitro skin equivalent model of GAS infection. (A-I) H\&E stained sections of uninfected $(A$ $C)$ or infected (D- $I$ ) wounded skin equivalents at 40x magnification; scale bar: $1000 \mu \mathrm{m}$. Uninfected wound 
at day $0(A)$ shows a lack of the epidermal layer where biopsy punch was performed, which healed by day 5 (B). At day 1 , infection of wounded skin equivalents with M3 and M41 GAS revealed superficial colonization of the wound bed, as well as invasion into the defects formed at the wound edge ( $D, G$, arrows). By day 5, bacteria had disseminated throughout tissue laterally and vertically (arrows), reaching the bottom of the dermis layer $(E, H)$. $(C, F, I) \mathrm{H} \& \mathrm{E}$ stained sections of the keratinocyte layer of intact skin surrounding the biopsy punch; scale bar: $100 \mu \mathrm{m}$. The thickness of the viable keratinocyte layer outside the wound $(C$, double arrow) was significantly reduced in tissues infected with M3 $(F)$ and M41 (I) by day 5. (J, L) Gram stained sections of wounded tissue, corresponding to boxed areas in panels $D$ and $H$, show tissue microcolonies (arrows) and superficial bacterial colonization (arrowheads). (J, scale bar: $400 \mu \mathrm{m}$; $L$, scale bar: $200 \mu \mathrm{m})$. (K, M) 1000x magnification micrographs of surface biofilms shown in $J$ and $L$ (scale bar: 10 $\mu \mathrm{m})$. ( $N-Q)$ Two-photon fluorescence microscopy analysis of infected skin equivalents. Vertical dissemination through the wound bed and glycocalyx formation by the GFP-expressing M41- $(N-O)$ and M3-type $(P-Q)$ GAS at day 5. 3D projections of z-stacks are shown from the top view of the z-stack (top panels) or the side view (bottom panels). Multi-channel images show GFP fluorescence of GAS cells ( $N$, $P)$ and TRITC-conA stain of glycocalyx $(O, Q)$. All images were acquired at $600 x$ magnification. 
Figure 5

A

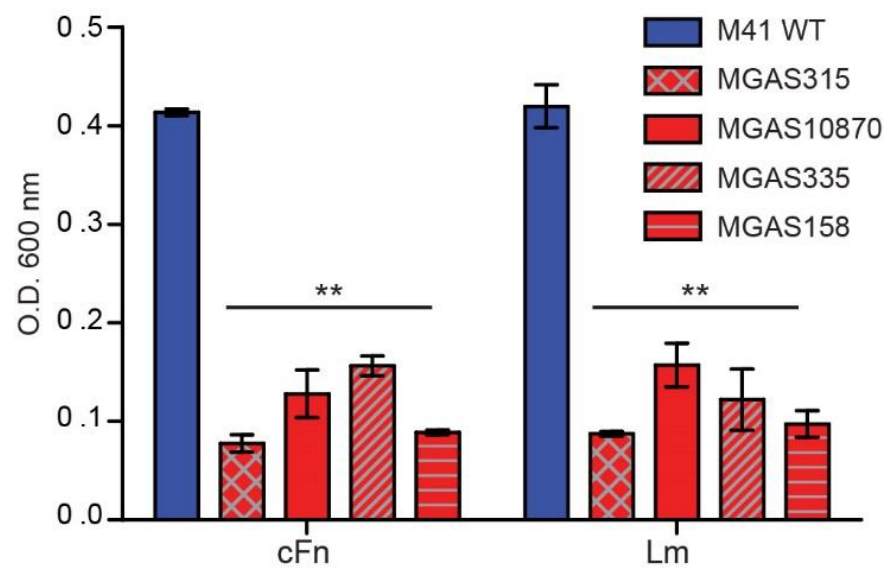

B

M3-type GAS

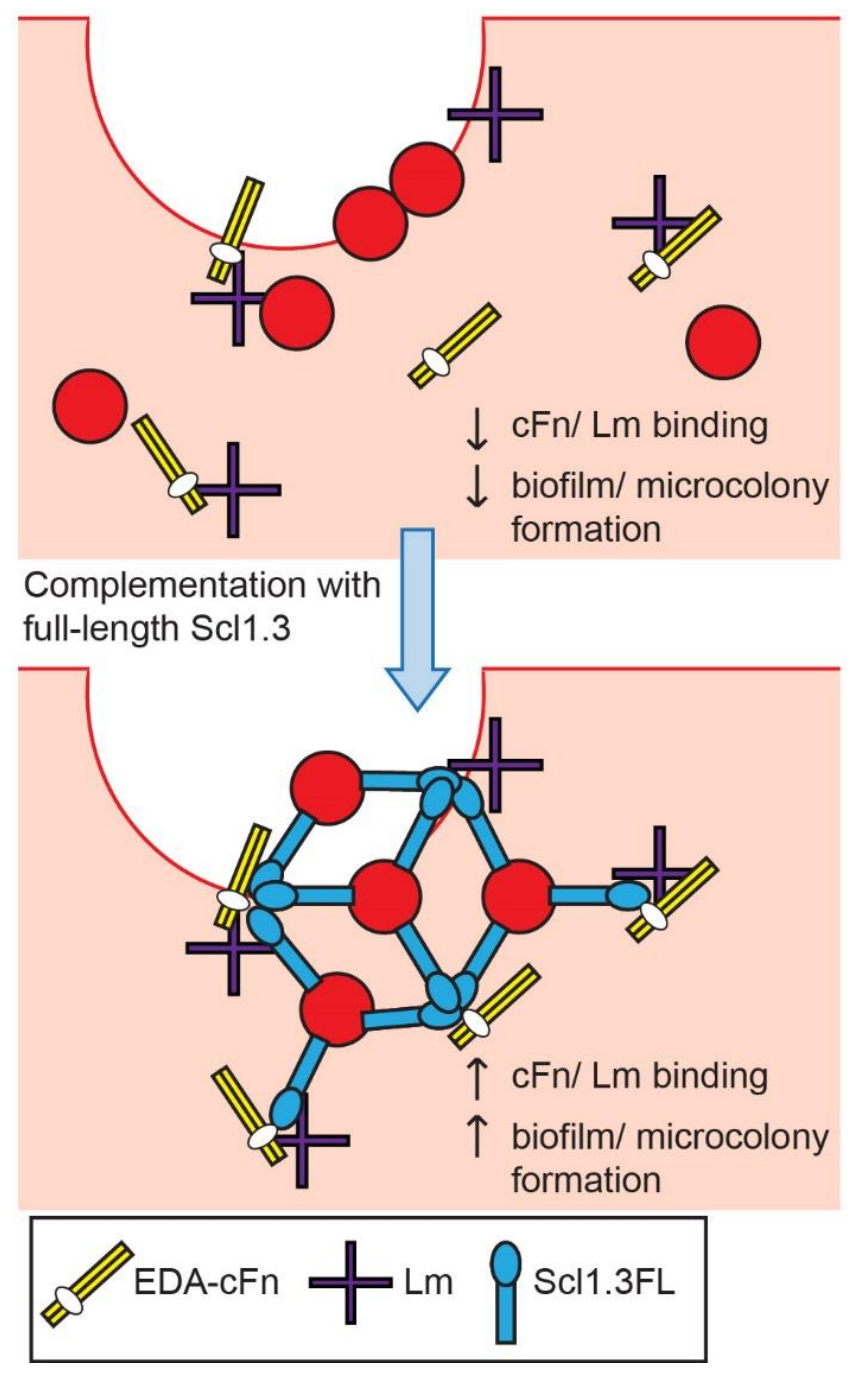


Figure 5: In vitro biofilm formation and hypothetical model of microcolony formation by invasive M3-type GAS. (A) Limited in vitro biofilm formation by invasive M3 strains on coatings with cellular fibronectin and laminin. A panel of invasive M3-type strains was compared to a non-invasive biofilm-capable M41-type strain MGAS 6183. Crystal violet staining was used to assess biomass formed after 24 hours of growth in wells coated with cellular fibronectin (cFn) and laminin ( $\mathrm{Lm})$; results represent averaged values from at least 3 independent experiments performed in triplicate wells. ${ }^{* *} \mathrm{P} \leq 0.01$ (student's $t$-test). (B) Hypothesis model. Top: Infection of wounded skin with wild-type M3-type GAS. Inherent lack of surfaceexpressed Scl1.3 causes decreased binding to cFn and Lm expressed in wounded tissue, and reduces biofilm and tissue microcolony formation by M3-type bacteria (red circles). Bottom: In-trans complementation of M3-type GAS with full-length cell-associated Scl1.3, Scl1.3FL, restores binding to cFn and $\mathrm{Lm}$ in tissue, which confers biofilm and tissue microcolony formation during infection. 


\section{Figure 6}
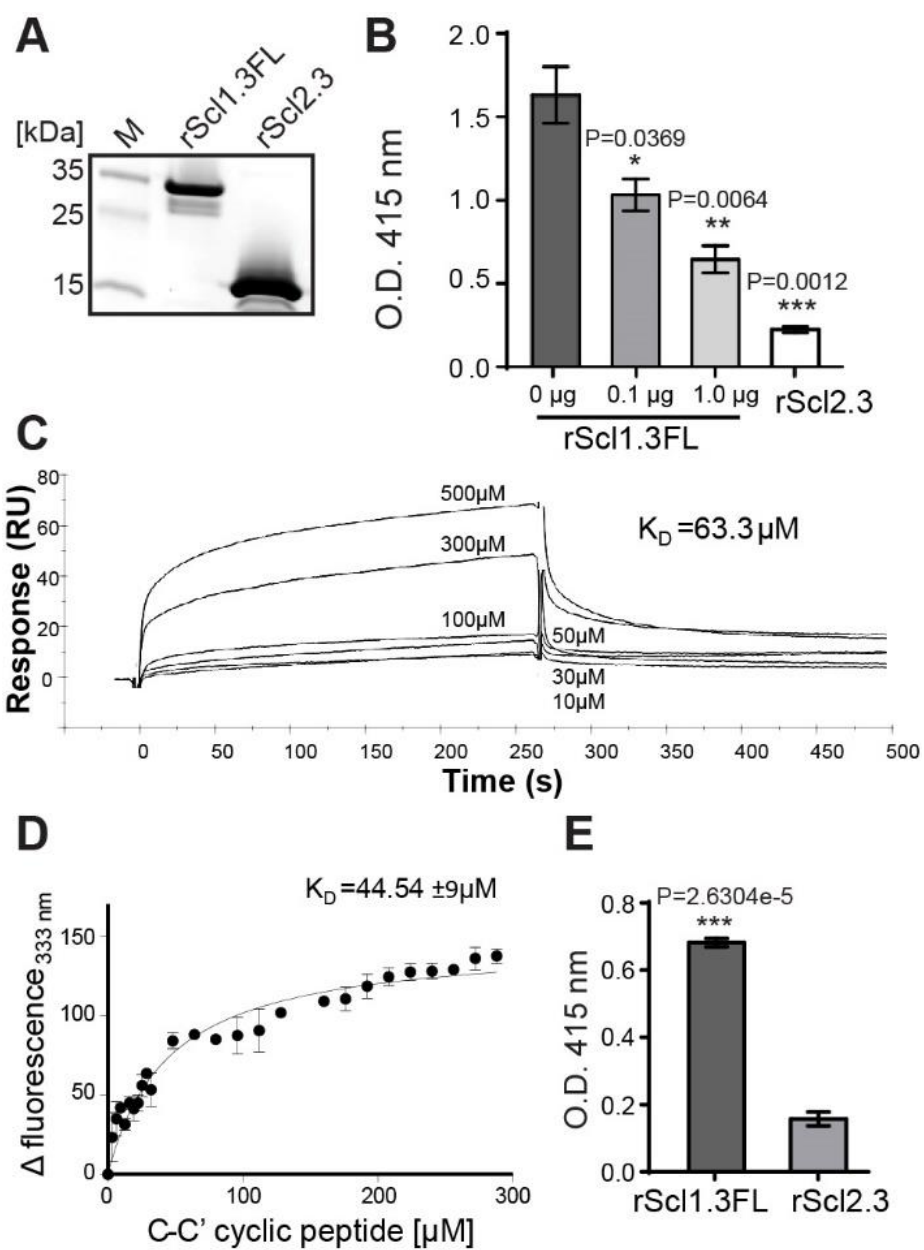

E

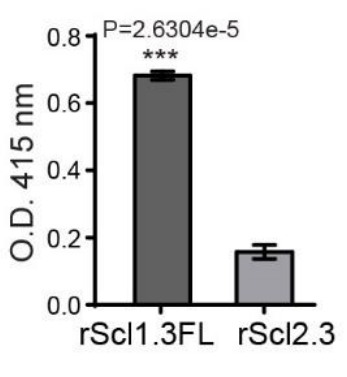

Figure 6: Construction and binding characterization of recombinant full-length Scl1.3FL and Scl2.3 proteins. (A) Affinity purified rScl1.3FL and rScl2.3 proteins were analyzed by $4-20 \%$ gradient SDS-PAGE; expected molecular masses: rScl1.3FL, $20.6 \mathrm{kDa}$ and rScl2.3, $16.2 \mathrm{kDa}$. (B-D-) Binding of rScl1.3FL to extra domain A (EDA) of cellular fibronectin. (B) IST-9 antibody inhibition identifies rScl1.3FL binding to the C-C' loop of EDA-cFN. Inhibition of rScl1.3FL binding to EDA-cFn was tested by ELISA following preincubation of cFn with blocking IST-9 mAb specific to the C-C' loop. Significance of inhibition by 0.1 and $1.0 \mu \mathrm{g}$ of IST-9 mAb was determined by student's $t$-test as compared to the untreated cFn. A lack of significant cFn binding by rScl2.3 was evident, as compared to rScl1.3FL-cFn binding; student's $t$-test. $(C)$ rScl1.3FL binding to a cyclic peptide mimicking the C-C' loop of EDA using surface plasmon resonance. Overlay of sensorgrams for the interaction between immobilized rScl1.3FL and EDA-derived C-C' cyclic peptide is shown. The experimental curves corresponding to different concentrations of peptide (10-500 $\mu \mathrm{M})$ were fitted according to a single binding model with 1:1 stoichiometry. (D) rScl1.3FL binding to a C-C' 
cyclic peptide using tryptophan fluorescence assay. Tryptophan fluorescence quenching analysis shows the dose-response curve of the fluorescence values of rScl1.3FL at $333 \mathrm{~nm}$ plotted against the concentration values of C-C' cyclic peptide. $(E)$ Laminin binding to rScl1.3FL and rScl2.3 by ELISA. Recombinant $\mathrm{rScl}$ proteins were immobilized onto Strep-Tactin-coated wells and incubated with laminin. Bound laminin was detected with specific primary pAbs and HRP-conjugated secondary Abs. Laminin binding was compared between rScl1.3FL and rSc2.3 and evaluated statistically using student's $t$-test. Results for panels $C$ and $F$ represent averaged values from at least 3 independent experiments performed in triplicate wells. ${ }^{*} \mathrm{P} \leq 0.05,{ }^{* *} \mathrm{P} \leq 0.01,{ }^{* *} \mathrm{P} \leq 0.001$. 
Figure 7
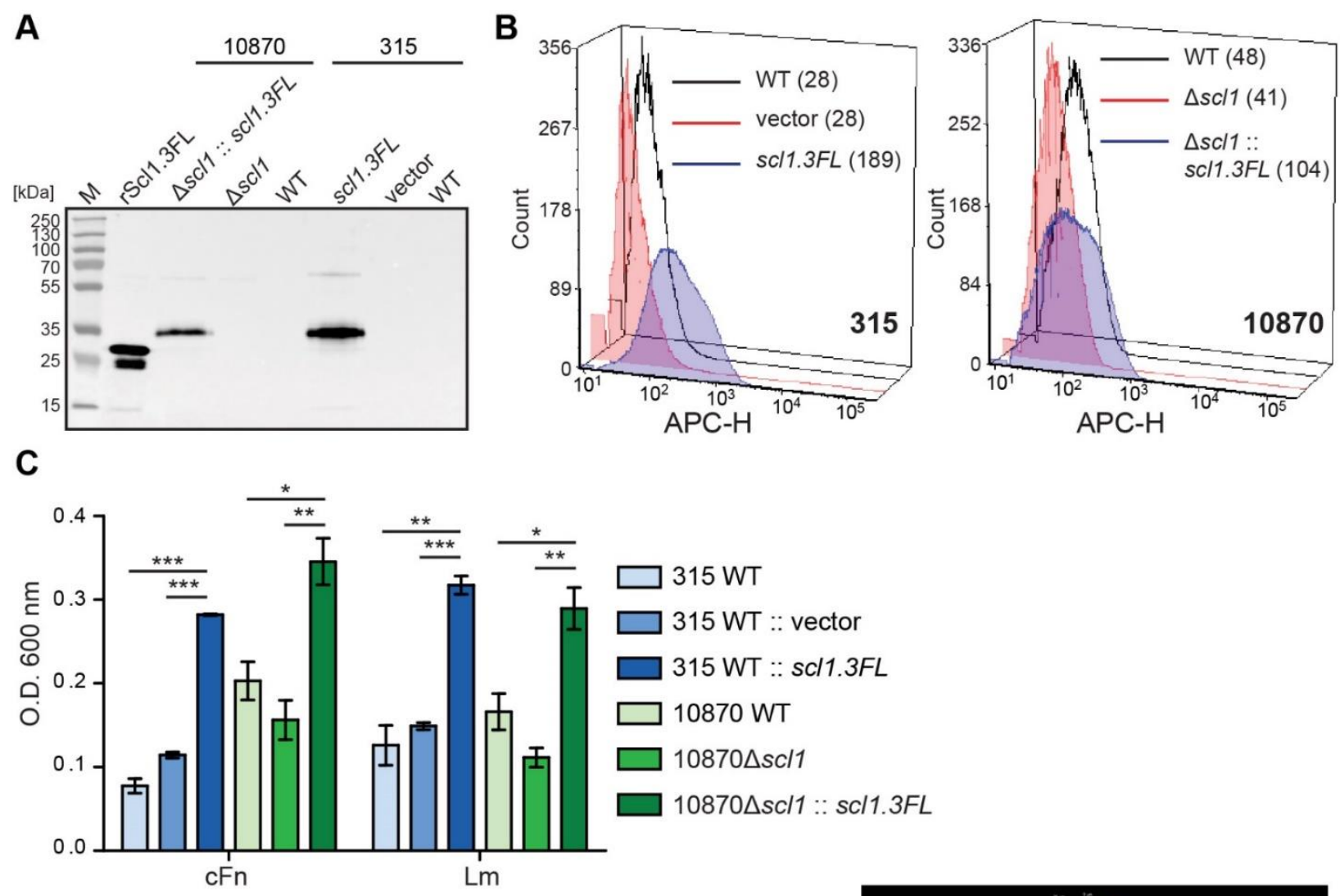

D
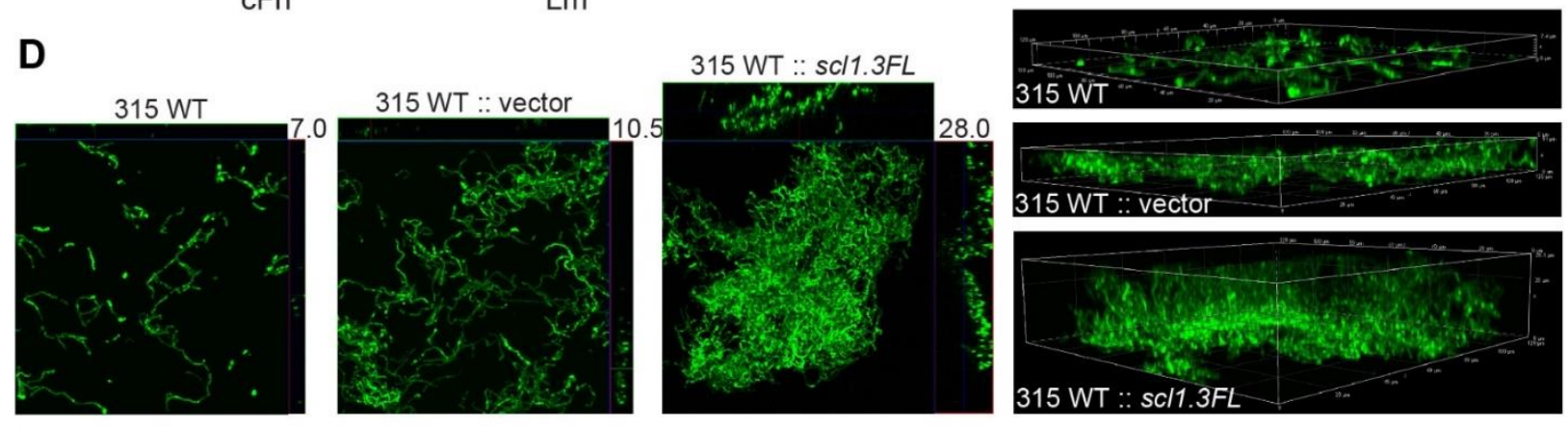

E
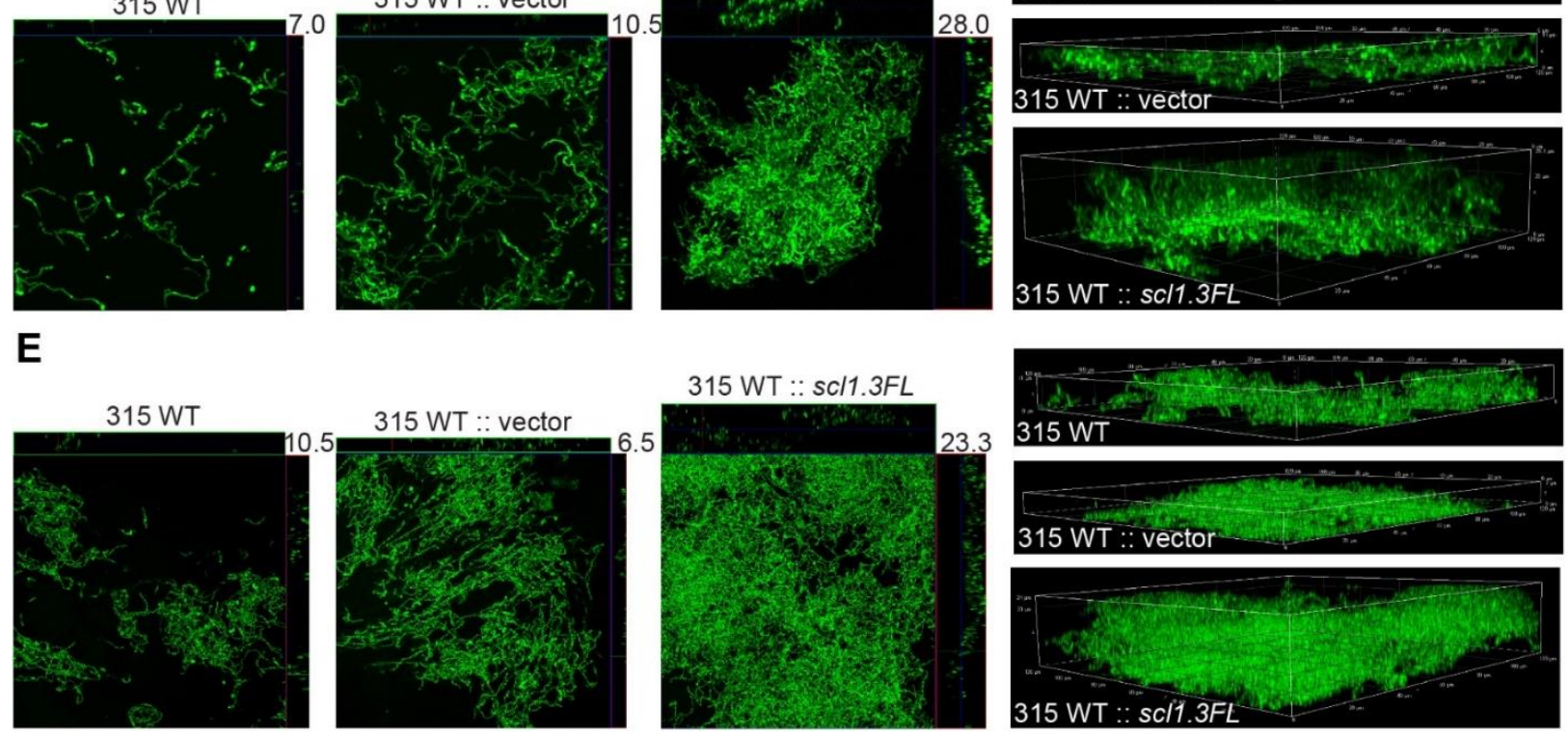

Figure 7: Homologous complementation of M3-type GAS with full-length surface-expressed Scl1.3 protein confers biofilm formation. $(A)$ Western blot detection of full-length Scl1.3 in cell wall fractions of 
MGAS315 and MGAS10870 $s$ scl1 complemented in-trans with the scl1.3FL allele. Parent strains were included as negative controls and rScl1.3FL was used as a positive control for detection. (B) Flow cytometry detection of Scl1.3FL on the GAS cell surface. Left: Fluorescence intensity of Scl1.3FL-complemented MGAS315 WT strain was compared to vector-complemented or WT parent strain. Right: Fluorescence intensity of Scl1.3FL-complemented MGAS10870 $\Delta s c / 1$ was compared to WT and $\Delta s c / 1$ parent strains. Median fluorescence intensities are shown in parentheses for each strain. $(C)$ Crystal violet assessment of bacterial biomass after 24 hours of growth in cFn- or Lm-coated wells. MGAS315 WT complemented intrans with pJRS525 $\left(\mathrm{Spc}^{\mathrm{R}}\right.$ vector) or pSL502 (Table 1) harboring the scl1.3FL allele was compared to WT parental strain. Spectinomycin resistant MGAS10870 $\Delta s c / 1$ mutant was complemented in-trans with $\operatorname{Kan}^{R}$ pSL518 (Table 1), harboring the scl1.3FL allele. Biofilm biomass of the complemented strain was compared to MGAS10870 WT and sc/1-inactivated parental strains. Results represent averaged values from at least 3 independent experiments performed in triplicate wells. ${ }^{*} \mathrm{P} \leq 0.05,{ }^{* *} \mathrm{P} \leq 0.01$, ${ }^{* * *} \mathrm{P} \leq 0.001$; students $t$-test. $(D, E)$ Confocal laser scanning microscopy analysis of biofilm formation by GFP-expressing MGAS315 vector and scl1.3FL- complemented strains. Biofilms were grown for 24 hours on cFn-coated $(D)$ or Lmcoated $(E)$ coverslips. Maximum intensity projections of GAS biofilms with cross-sectional views (left panels) are representative of $z$-stacks from ten fields within a single experiment. Average vertical thickness is indicated in micrometers. 3D projections of z-stacks (right panels) are shown from the side view. 


\section{Figure 8}
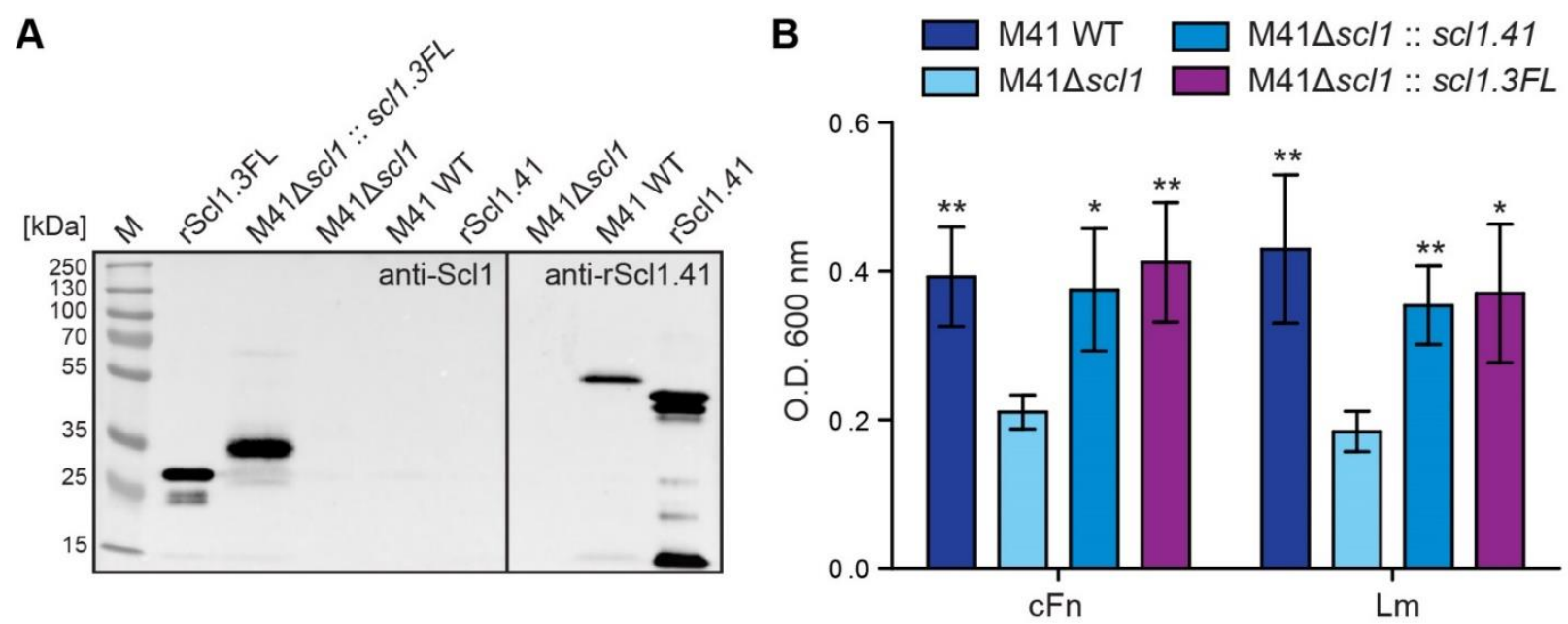

C

D

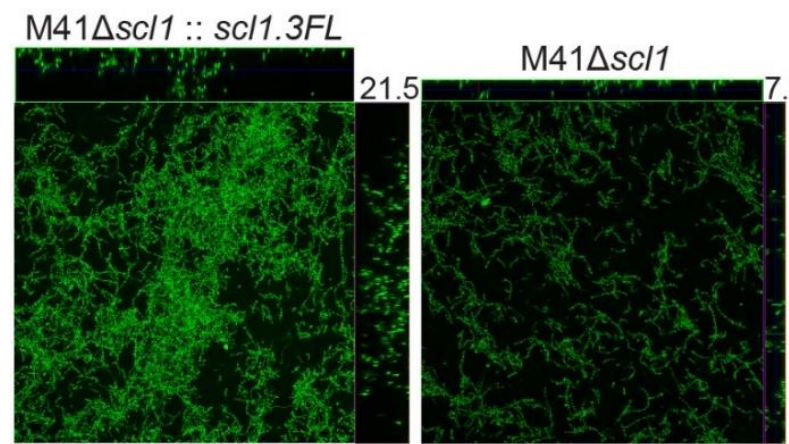

M41 $s c / 1:: s c / 1.3 F L$
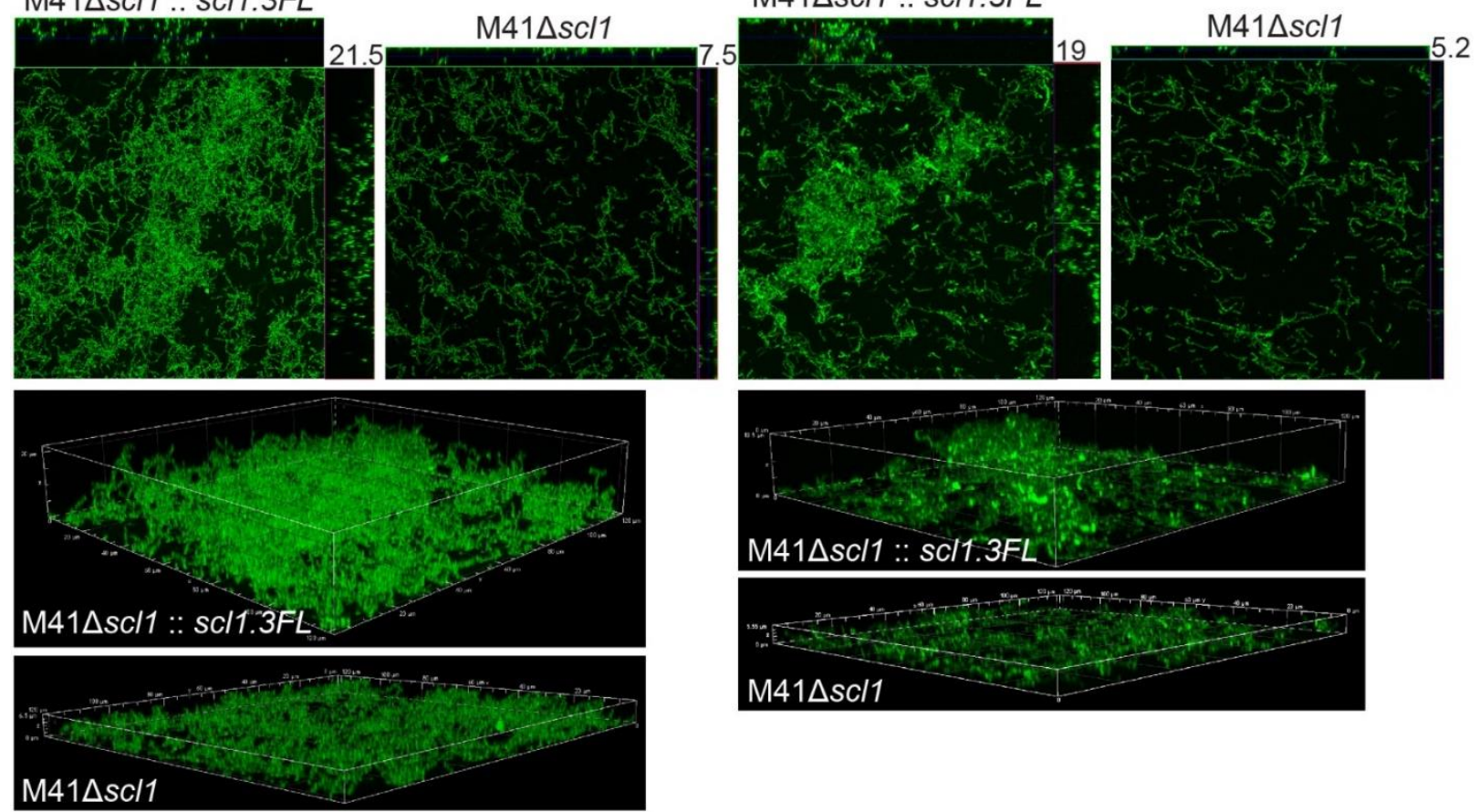

Figure 8: Heterologous complementation of scl1-deficient mutant of M41-type GAS with full-length Scl1.3 restores biofilm formation. $(A)$ Left blot: western blot detection of full-length Scl1.3 protein in cell wall fraction of M41 $\Delta s c / 1:: s c / 1.3 F L$ using anti-Scl1 polyclonal antibody. The M41 1 sc/1 and M41 WT strains were included as negative controls, and rScl1.3FL was included as a positive control. Right blot: western blot detection of Scl1.41 in M41 $\Delta s c / 1$ and M41 WT strains using anti-rScl1.41 antibody and rScl1.41 as a positive control. (B) Crystal violet assessment of biofilm biomass after 24 hours of growth in cFn- or Lm- 
coated wells. M41 sc/1-inactivated (M41 $\Delta s c / 1)$ mutant was complemented in-trans with either homologous scl1.41 allele harbored on pSL230 or with heterologous scl1.3FL allele harbored on pSL502 (Table 1). M41 WT, $\Delta s c / 1$ mutant and two complemented mutant strains were assessed for biofilm formation and statistical significance was assessed by students $t$-test as compared to $\mathrm{M} 41 \Delta s c / 1 ;{ }^{*} \mathrm{P} \leq 0.05,{ }^{* *} \mathrm{P} \leq 0.01$. (C, D) Confocal laser scanning microscopy analysis of biofilm formation by GFP-expressing M41 $\Delta s c / 1$ or M41_sc/1:: sc/1.3FL. Biofilms were grown for 24 hours on cFn-coated (C) or Lm-coated $(D)$ coverslips. Maximum intensity projections of GAS biofilms with cross-sectional views (top panels) are representative of z-stacks from ten fields within a single experiment. Average vertical thickness is indicated in micrometers. 3D projections of z-stacks (bottom panels) are shown from the side view. 
Figure 9

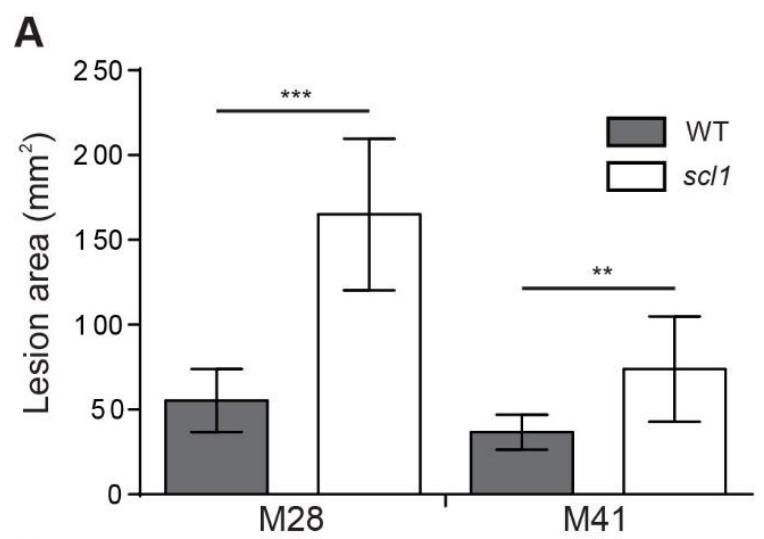

B

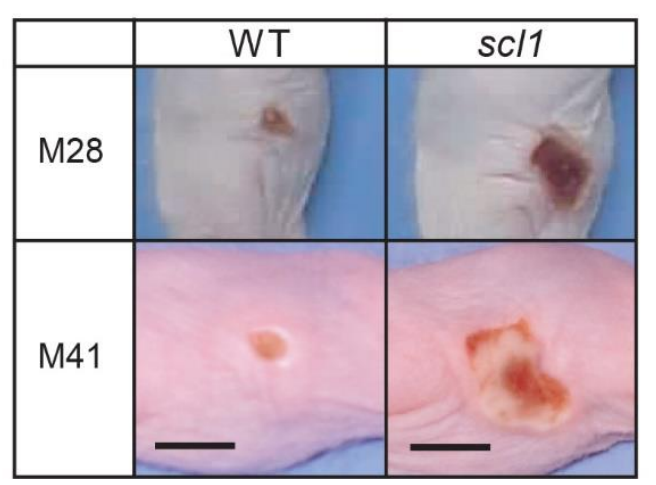

Figure 9: Skin pathology of SKH1 hairless mice infected subcutaneously with wild-type and scl1inactivated mutants of M28- and M41-type GAS. $(A)$ Mean area of skin lesions in mice infected with M28or M41-type isogenic GAS strains. The severity of skin lesions was compared following infection with wildtype (WT) or sc/1-mutant (sc/1) GAS; 96-hour time-point is shown. The mean lesion area and standard deviation were calculated and compared between experimental groups. Data represents mean lesion size from at least 10 mice per experimental group. Statistical differences were calculated using the student's $t$ test; ${ }^{* *} P \leq 0.01,{ }^{* * *} P \leq 0.001$. (B) Gross pathology of the skin lesions. Digital images of the skin lesions of representative animals infected with either WT or sc/1-mutant GAS. The images show skin lesions developed after 96 hours post-infection, and scale bar represents $10 \mathrm{~mm}$. 
Figure 10

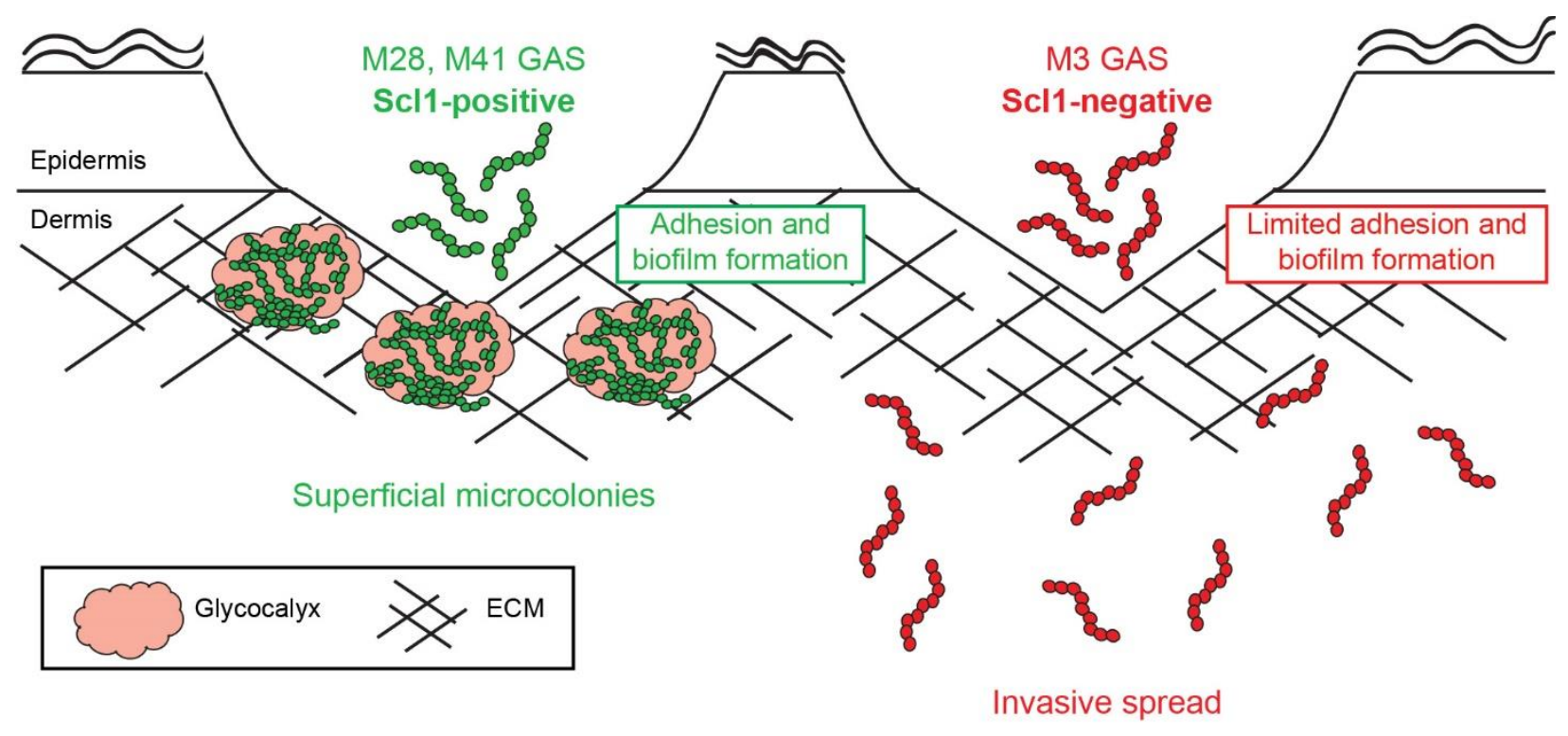

Figure 10: Model of Scl1-mediated GAS adhesion, biofilm formation, and host colonization. M28and M41-type GAS express full-length cell-attached Scl1, which mediates adherence to cFn and Lm. ECM binding promotes biofilm formation in wounded tissue and allows the formation of superficial tissue microcolonies resulting in non-invasive colonization. On the contrary, in M3-type GAS, naturally lacking fulllength cell-attached Scl1.3 adhesin, bacterial cells have reduced adherence to cFn and Lm and reduced capacity for biofilm formation, thereby circumventing the formation of localized tissue microcolonies; infection balance is shifted towards dissemination through tissue, promoting invasive spread. 


\section{Supplemental Figure 1}
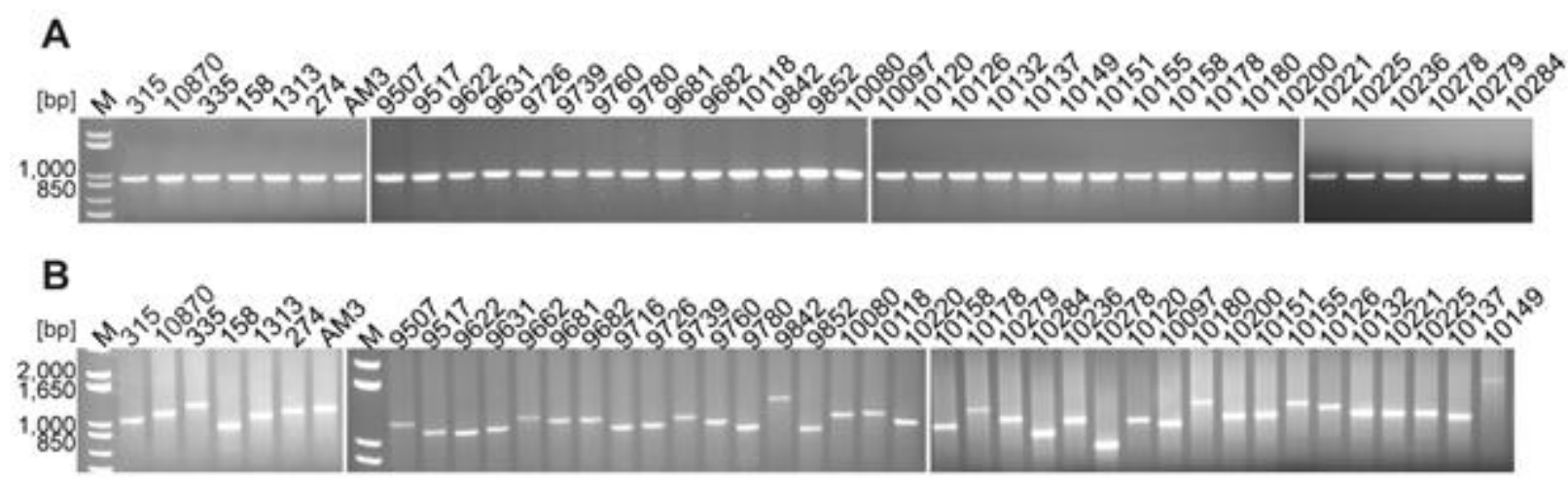

Figure S1. Analytical PCR of scl1.3 and scl2.3 in M3-type GAS. (A) PCR of sc/1.3 in 42 M3-type strains was performed with primers 232Up and 232Rev flanking the scl1 locus (Table S1). Expected size based on MGAS315: 1,010 bp. M, 1 kb Plus DNA Ladder. (B) PCR of scl2.3 in 42 M3-type strains was performed with primers Scl2.3 F and Scl2.3 R flanking the scl2.3 collagen-like region (Table S1). Expected size based on MGAS315 genome is 1,125 bp. M, $1 \mathrm{~kb}$ Plus DNA Ladder. MGAS designation applies to all strain numbers shown above gel wells, with the exception of strain AM3. 


\section{Supplemental Figure 2}

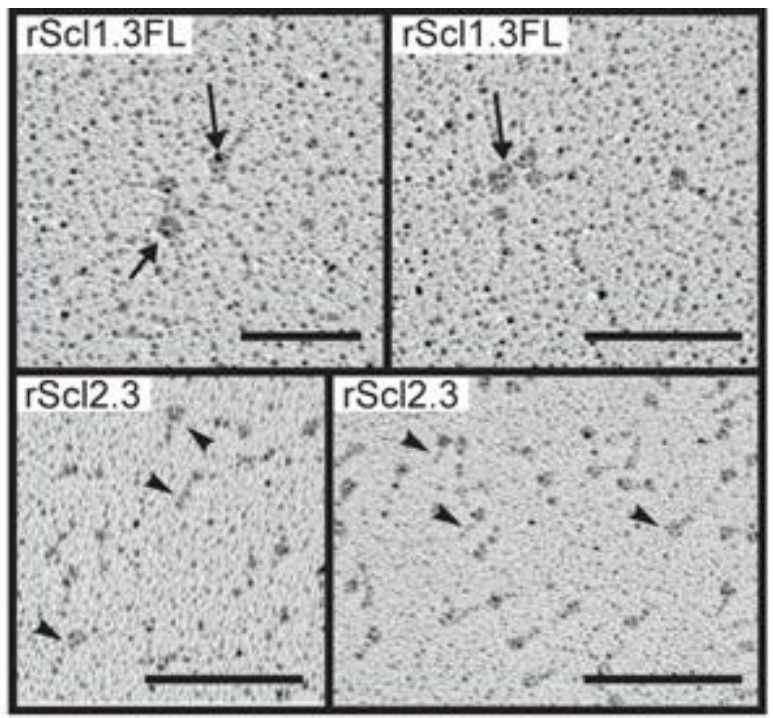

Figure S2. Domain organization of rScl1.3FL and rScl2.3 proteins. Rotary shadowing of rScl proteins demonstrates the conserved lollipop-like domain organization. Aggregates mediated by the interactions between $\mathrm{V}$ regions of $\mathrm{rScl1}$.3FL are seen in the top panels (depicted by arrows). No apparent aggregation was observed in the rScl2.3 preparation (single lollipops are depicted by arrowheads). All scale bars reflect $100 \mathrm{~nm}$. 


\section{Supplementary Figure 3}

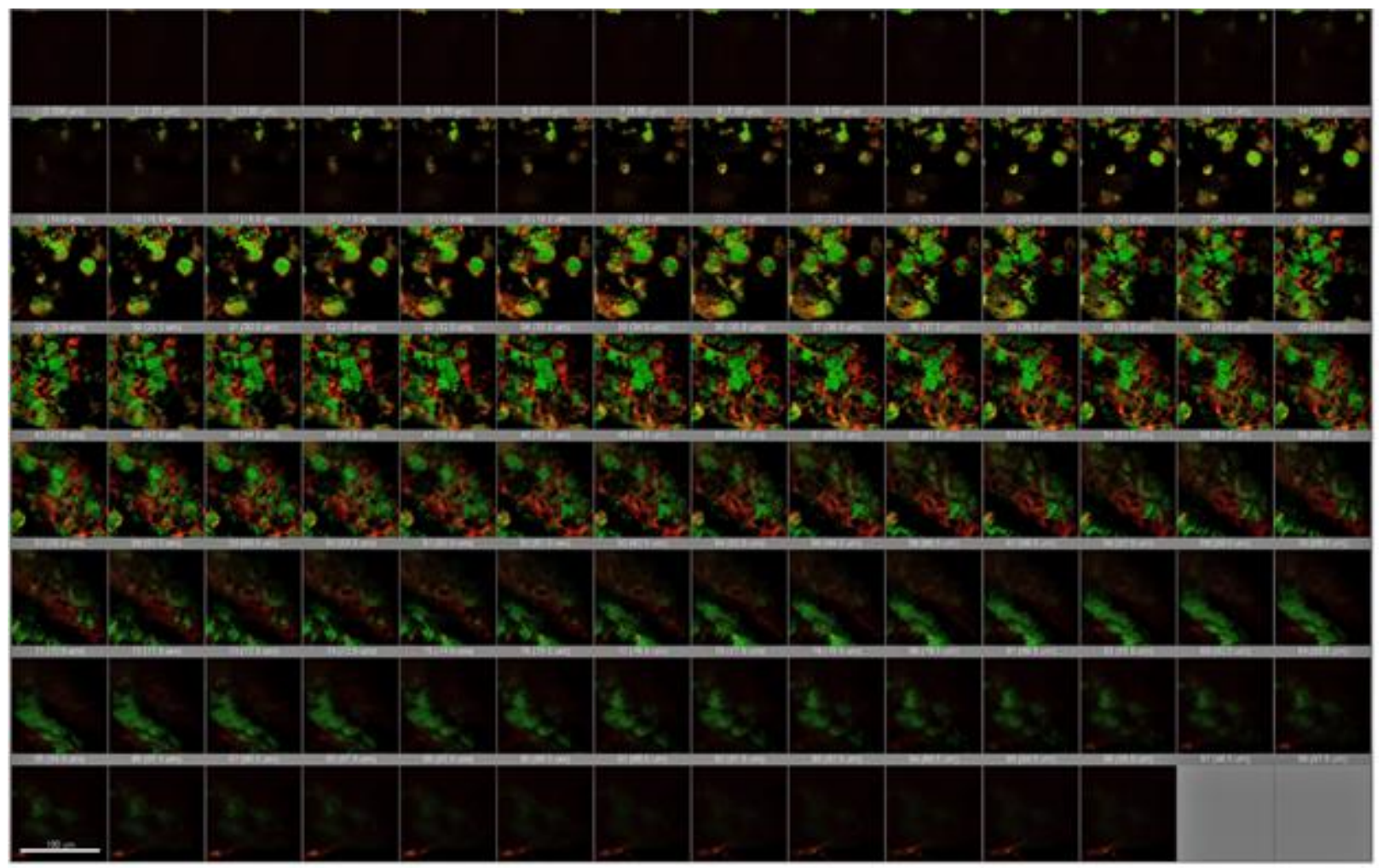

Figure S3. Colocalization of glycocalyx staining with GFP-expressing M41-type GAS during in vitro infection of skin equivalent. Representative TPF gallery image of z-stack layers from 3D projection (Figure 5 of manuscript), showing colocalization of TRITC-concanavalin A staining of glycocalyx (red) with GFP-expressing GAS microcolonies (green). Z-stack step size: $1 \mu \mathrm{m} ; 600 x$ magnification. 


\section{Supplementary Figure 4}

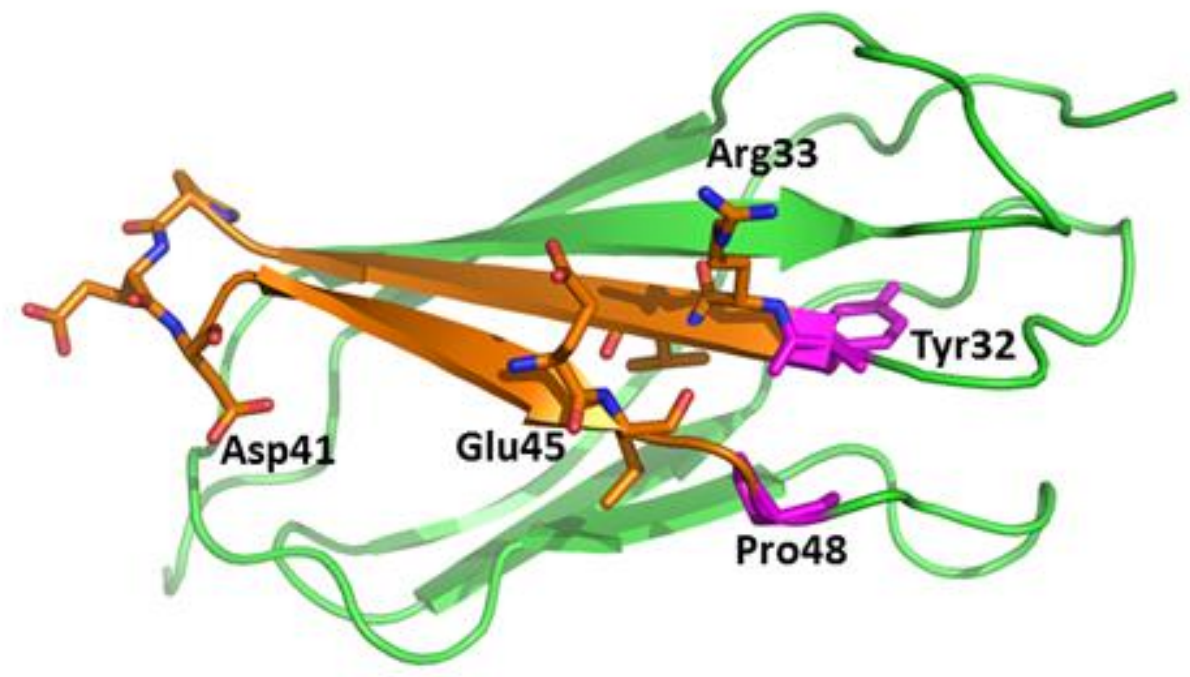

Figure S4. Strategy for C-C' loop peptide design. Cartoon model of the EDA domain of cFn (PDB code $1 \mathrm{~J} 8 \mathrm{~K}$ ) used for the $\mathrm{C}-\mathrm{C}^{\prime}$ peptide design. The designed peptide includes the $\mathrm{C}-\mathrm{C}$ ' beta-hairpin (orange). The terminal residues of the beta-hairpin (magenta) were mutated to cysteine to induce a disulfide bond and mimic the conformation of the peptide in the protein. The key residue for recognition, Asp41, is reported in ball-and-stick along with the residues Arg33 and Glu45, which form a salt bridge, and the terminal residues mutated to cysteine. 


\section{TABLES}

Table 1. Constructs used in this study

\begin{tabular}{lll}
\hline Plasmid & Description & Source \\
\hline pJRS525 & E. coli / S. pyogenes shuttle vector & Mclver and Scott. 1997 \\
pSL230 & pJRS525 with scl1.41 allele & Caswell et al. 2007 \\
pSL501 & pJRS525 with scl1.3WT allele & Flores et al. 2015 \\
pSL502 & pSL501 with repaired allele scl1.3FL & Flores et al. 2015 \\
pSL518 & pSL502 with Kan ${ }^{\mathrm{R}}$ in place of Spc ${ }^{\mathrm{R}}$ & This study \\
pASK-IBA2 & E. coli expression vector & IBA, Göttingen \\
pSL154 & pASK-IBA2 encoding rScl1.3V & This study \\
pSL503 & pASK-IBA2 encoding rScl1.3FL & Flores et al. 2015 \\
pSL514 & pASK-IBA2 with rScl2.3 & This study \\
pSB027 & GFP-encoding plasmid & Cramer et al. 2003 \\
\hline
\end{tabular}


Supplementary Table 1: Primers used in this study

\begin{tabular}{|c|c|c|}
\hline Primer name & Sequence (5'-3') & Use \\
\hline \multicolumn{3}{|l|}{ Analytical PCR } \\
\hline IS1548F & GCCGTCTGCGTGCCCATTGCGTCTA & Detection of \\
\hline Scl1 R & ACTAGATCTGAGATTATGGTGCTTTGATGTC & IS1548 \\
\hline 232 Up & CTCCACAAAAGAGTGATCAGTC & Amplification \\
\hline 232 Rev & TTAGTTGTTTTCTTTGGCGTTT & of $s c / 1.3$ \\
\hline $\mathrm{Scl} 2.3 \mathrm{~F}$ & AGGCATACAAGATCATGTCCTTGA & Amplification \\
\hline Scl2.3 R & TTTGGTGTATGTGGTGCGGT & of $s c / 2.3$ \\
\hline Scl Up & CTTTCAATGGATGACGATACC & Amplification \\
\hline Scl Rev & ACTTTCCATCAGTTAGGTAGC & of $s c / 2.3$ \\
\hline \multicolumn{3}{|l|}{ Cloning } \\
\hline Scl1.3 M3VF & GAGATGGCCGAGACTCCTATGACATCAAAGG & Cloning of \\
\hline Scl1.3 M3VR & $\begin{array}{l}\text { CAGCGTCTCAGCGCTCTTTGTTGCACCTTTTTCAA } \\
\text { TCAG }\end{array}$ & $\begin{array}{l}s c / 1.3 \mathrm{~V} \\
\text { region }\end{array}$ \\
\hline 232 Up & CTCCACAAAAGAGTGATCAGTC & Cloning of \\
\hline ME7 & TCAGTGAATTCTCTTTAGAGGATTAG & scl1.3WT \\
\hline pJRS525F1 & GGGTTTTCCCAGTCACG & Repair of \\
\hline ME6 $^{a}$ & TCCAGCAGGACCTCGAGGTGAACGC & scl1.3 null \\
\hline Scl1.3 M3VF & GAGATGGCCGAGACTCCTATGACATCAAAGG & Cloning of \\
\hline Scl1.28WMR & $\begin{array}{l}\text { GTCAAGCTTATTATTTTTCGAACTGCGGGTGGCTC } \\
\text { CAAGGTTTTTCTGGAGCTGGAGTTACC }\end{array}$ & $s c / 1.3 F L$ \\
\hline Scl1.3 M3VF ext & $\begin{array}{l}\text { GAGATGGCCGAGACTCCTATGACATCAAAGGAGA } \\
\text { GACAAG }\end{array}$ & $\begin{array}{l}\text { Cloning of } \\
\text { scl1.3WT }\end{array}$ \\
\hline Scl1.3 truncR2 & $\begin{array}{l}\text { GGTCTCAGCGCTACCTCGAGGTCCTGCTGGACCT } \\
\text { TG }\end{array}$ & \\
\hline $\begin{array}{l}\text { Scl2 M3VF } \\
\text { Scl2.28_WMR }\end{array}$ & $\begin{array}{l}\text { GAGATGGCCGATGGTGAAGATGCCCAAAAAAG } \\
\text { GTCAAGCTTATTATTTTTCGAACTGCGGGTGGCTC } \\
\text { CAT }\end{array}$ & $\begin{array}{l}\text { Cloning of } \\
\text { scl2.3 }\end{array}$ \\
\hline \multicolumn{3}{|l|}{ qRT-PCR analysis } \\
\hline tufA_F & CAACTCGTCACTATGCGCACAT & qRT-PCR of \\
\hline tufA_R & GAGCGGCACCAGTGATCAT & tufA \\
\hline Scl1_WMR_exp_F & TGCTGACAAAGAAGCTAACCAAAC & qRT-PCR of \\
\hline Scl1_WMR_exp_R & GTGGTTGTTGGCTACAGGTGTCT & $s c / 1$ \\
\hline Scl2_WMR_exp_F & TCCTAAAACACCAGAGGTCC & qRT-PCR of \\
\hline Scl2_WMR_exp_R2 & TGTGTGTGTCGTGAGCTGC & scl2 \\
\hline emm3_exp_F2 & AACAAATCTCAGACGCAAGCCGTC & qRT-PCR of \\
\hline emm3_exp_R2 & TTCAAGCTCTTTGTTWAGTTTTTCAAG & emm \\
\hline Mga_TMF & CAAGTCAACAGTGGAGAGAACTAAAATT & qRT-PCR of \\
\hline Mga_TMR & ATGGAGATGTTGAGAGCTTTGCT & mga \\
\hline Mga_F1 & ATCAGACAAAAACATTAAATTGCATG & Sequencing \\
\hline Mga R1 & TTGCATGTTAGTGAGACAAGTTTGC & of $\mathrm{mga}$ \\
\hline
\end{tabular}


Supplementary Table 2: Variation in sc/2.3 gene among M3 strains

\begin{tabular}{|c|c|c|c|c|c|}
\hline Strain & $\begin{array}{l}\text { No. of } \mathrm{GXY} \\
\text { repeats }\end{array}$ & $\begin{array}{l}\text { No. of bp in the } \\
\text { CL region }\end{array}$ & $\begin{array}{l}\text { Amplicon size } \\
\text { (bp) }{ }^{a}\end{array}$ & $\begin{array}{l}\text { No. of } \\
\text { CAAAA } \\
\text { repeats }\end{array}$ & $\begin{array}{l}\text { GTG in } \\
\text { frame }^{\text {b }}\end{array}$ \\
\hline MGAS274 & $\sim 125$ & $\sim 1125$ & $\sim 1206$ & 8 & yes \\
\hline MGAS315 & 116 & 1044 & 1125 & 5 & yes \\
\hline MGAS335 & $\sim 135$ & $\sim 1215$ & $\sim 1296$ & 4 & no \\
\hline MGAS1313 & $\sim 119$ & $\sim 1071$ & $\sim 1152$ & 8 & yes \\
\hline AM3 & $\sim 129$ & $\sim 1161$ & $\sim 1242$ & 10 & no \\
\hline MGAS3375 & 17 & 153 & 234 & 5 & yes \\
\hline MGAS9517 & 113 & 1017 & 1098 & 8 & yes \\
\hline MGAS9622 & 111 & 999 & 1080 & 11 & yes \\
\hline MGAS9631 & 113 & 1017 & 1098 & 18 & no \\
\hline MGAS9716 & 110 & 990 & 1071 & 14 & yes \\
\hline MGAS9726 & 111 & 999 & 1080 & 11 & yes \\
\hline MGAS9739 & 119 & 1071 & 1152 & 5 & yes \\
\hline MGAS9760 & 113 & 1017 & 1098 & 11 & yes \\
\hline MGAS9780 & 106 & 954 & 1035 & 14 & yes \\
\hline MGAS9842 & 139 & 1251 & 1332 & 9 & no \\
\hline MGAS9852 & 103 & 927 & 1008 & 8 & yes \\
\hline MGAS10080 & 117 & 1053 & 1134 & 5 & yes \\
\hline MGAS10118 & 119 & 1071 & 1152 & 5 & yes \\
\hline MGAS10220 & 110 & 990 & 1071 & 12 & no \\
\hline
\end{tabular}

${ }^{a}$ Amplicons were generated using primers Scl2.3 F/ R (Table S1)

${ }^{b}$ GTG is scl2 start codon 
Supplementary Table 3: Mass spectrometry identification of rScl and native Scl proteins $^{\mathrm{a}}$

\begin{tabular}{|c|c|c|c|c|c|}
\hline Sample & $\begin{array}{l}\text { No. of } \\
\text { peptides } \\
>95 \% \\
\text { confident }\end{array}$ & Peptide & Confidence & $\begin{array}{l}\text { Sequence } \\
\text { coverage }\end{array}$ & $\begin{array}{l}\text { Confident } \\
\text { ID }\end{array}$ \\
\hline rScl1.3V & 4 & $\begin{array}{l}\text { EENSQEELK } \\
\text { EENSQEELKNFTEER } \\
\text { LKEILDLIEK } \\
\text { WYGTYFKEENSQEELK }\end{array}$ & $\begin{array}{l}99 \% \\
99 \% \\
99 \% \\
99 \%\end{array}$ & $40 \%$ & yes \\
\hline rScl2.3 & 2 & $\begin{array}{l}\text { GIQDHVLDGQDGDR } \\
\text { EELLSALIDGTSR }\end{array}$ & $\begin{array}{l}99 \% \\
98.13 \%\end{array}$ & $18 \%$ & yes \\
\hline \multicolumn{6}{|l|}{ MGAS315 WT } \\
\hline Scl2.3 in Sup & 12 & $\begin{array}{l}\text { DVTPAPQNPSN } \\
\text { DVTPAPQNPSNR } \\
\text { EELLSALIDGTSR } \\
\text { GEAGPAGPR } \\
\text { GIQDHVLDGQDGDR } \\
\text { GIQDHVLDGQDGDRGEAGPA } \\
\text { GPR } \\
\text { GLNKPQTQGGNQL } \\
\text { GLNKPQTQGGNQLAK } \\
\text { NKPQTQGGNQLAK } \\
\text { REELLSALIDGTSR } \\
\text { TPEVPQKPDTAPHTPK } \\
\text { TPQIPGQSK }\end{array}$ & $\begin{array}{l}99 \% \\
99 \% \\
99 \% \\
99 \% \\
99 \% \\
99 \% \\
\\
99 \% \\
99 \% \\
99 \% \\
99 \% \\
99 \% \\
99 \%\end{array}$ & $17 \%$ & yes \\
\hline Scl2.3 in CW & 7 & $\begin{array}{l}\text { DVTPAPQNPSNR } \\
\text { EELLSALIDGTSR } \\
\text { GIQDHVLDGQDGDR } \\
\text { GIQDHVLDGQDGDRGEAGPA } \\
\text { GPR } \\
\text { GLNKPQTQGGNQLAK } \\
\text { REELLSALIDGTSR } \\
\text { TPEVPQKPDTAPHTPK }\end{array}$ & $\begin{array}{l}99 \% \\
99 \% \\
99 \% \\
99 \% \\
\\
99 \% \\
99 \% \\
99 \%\end{array}$ & $15 \%$ & yes \\
\hline \multicolumn{6}{|c|}{ Scl1.3FL- complemented GAS } \\
\hline $\begin{array}{l}\text { 315WT :: } \\
\text { scl1.3FL }\end{array}$ & 5 & $\begin{array}{l}\text { EENSQEELKNFTEER } \\
\text { EILDLIEK } \\
\text { GDKGETGLAGPVGPAGK } \\
\text { GETGLAGPVGPAGK } \\
\text { LKEILDLIEK }\end{array}$ & $\begin{array}{l}99 \% \\
99 \% \\
99 \% \\
99 \% \\
99 \%\end{array}$ & $17 \%$ & yes \\
\hline $\begin{array}{l}10870 \Delta s c / 1:: \\
s c / 1.3 F L\end{array}$ & 4 & $\begin{array}{l}\text { EILDLIEK } \\
\text { GDKGETGLAGPVGPAGK } \\
\text { GETGLAGPVGPAGK } \\
\text { LKEILDLIEK }\end{array}$ & $\begin{array}{l}99 \% \\
99 \% \\
99 \% \\
99 \%\end{array}$ & $11 \%$ & yes \\
\hline $\begin{array}{l}\text { M41 } \Delta s c / 1:: \\
s c / 1.3 F L\end{array}$ & 3 & $\begin{array}{l}\text { EENSQEELKNFTEER } \\
\text { GDKGETGLAGPVGPAGK } \\
\text { GETGLAGPVGPAGK }\end{array}$ & $\begin{array}{l}99 \% \\
99 \% \\
99 \%\end{array}$ & $13 \%$ & yes \\
\hline
\end{tabular}


Supplementary Table 4: Summary table of fold-changes in scl1 and scl2 expression $^{a}$

\begin{tabular}{lllllll}
\hline Strain & Gene & Fold change & + SE & - SE & P value & Significance \\
\hline M1 & $s c / 1$ & 21.05195084 & -2.33583 & -2.62734 & 0.001184755 & $* *$ \\
& $s c / 2$ & -23.6920597 & -1.97551 & -1.82347 & 0.01549836 & $*$ \\
M28 & $s c / 1$ & 6.237987683 & -1.1098 & -1.34997 & 0.01395444 & $*$ \\
& $s c / 2$ & -7.25032516 & 0.960352 & 1.106978 & 0.034822349 & $*$ \\
M41 & $s c / 1$ & 7.787441864 & -0.82018 & -0.91673 & 0.004897482 & $* *$ \\
& $s c / 2$ & -3.22615419 & 0.376259 & 0.425935 & 0.141911016 & N.S. \\
\multicolumn{2}{l}{ M3 MGAS: } & & & & & \\
10870 & $s c / 1$ & -25.2160816 & 1.366976 & 1.445328 & $5.48581 E-06$ & $* *$ \\
& $s c / 2$ & -1.84753589 & -0.01174 & -0.01166 & 0.077646106 & N.S. \\
158 & $s c / 1$ & -45.8489636 & -0.86051 & -0.84465 & 0.000121468 & $* * *$ \\
& $s c / 2$ & 1.077129939 & 0.059307 & 0.056212 & 0.753558444 & N.S. \\
335 & $s c / 1$ & -42.9309881 & -4.03211 & -3.68593 & $5.18378 E-05$ & $* \star *$ \\
& $s c / 2$ & -2.44408041 & -1.65928 & -0.98832 & 0.283398851 & N.S. \\
1313 & $s c / 1$ & -43.929358 & 0.938527 & 0.959016 & $5.45812 E-06$ & $* * *$ \\
& $s c / 2$ & -3.89390614 & -2.38312 & -1.47835 & 0.117229672 & N.S. \\
\hline
\end{tabular}

aFold-changes in scl1 and scl2 expression relative to M3 MGAS315 are shown for each strain. Data is based on qRT-PCR analysis of RNA isolated during exponential growth phase and expression is normalized to the expression of tufA gene. +SE, positive standard error; -SE, negative standard error. Significance is calculated based on three independent experiments, each performed in triplicate wells. ${ }^{*} P<0.05,{ }^{* *} P<0.01$, ${ }^{* * *} P<0.001$; student's $t$-test. 